\title{
The wasp genus Sphex in Sub-Saharan Africa (Hymenoptera: Sphecidae)
}

\author{
Thorleif H. DÖRFEL ${ }^{1, *}$ \& Michael OHL ${ }^{\circledR 2}$ \\ Museum für Naturkunde, Invalidenstraße 43, 10115 Berlin, Germany. \\ "Corresponding author: thorleif.doerfel@mfn.berlin \\ ${ }^{2}$ Email: michael.ohl@mfn.berlin \\ ${ }^{1}$ urn:1sid:zoobank.org:author:11B5C093-23D5-417C-AB64-65764FC2AF05 \\ ${ }^{2}$ urn:1sid:zoobank.org:author:878259F2-C3C6-4264-B04A-C397E01E5C8E
}

\begin{abstract}
The Afrotropical species of the sphecid genus Sphex are revised. Forty-six taxa are recognized, of which fifteen are newly described: Sphex abbotti nivarius subsp. nov., S. comorensis sp. nov., S. hades sp. nov., S. nefrens sp. nov., S. occidentalis sp. nov., S. pseudopraedator sp. nov., S. pseudosatanas sp. nov., S. pulawskii sp. nov., S. rufoclypeatus sp. nov., S. satanas memnon subsp. nov., S. schmideggeri sp. nov., S. schoutedeni malawicus subsp. nov., S. socotrensis sp. nov., S. stadelmanni rufus subsp. nov. and $S$. victoria sp. nov. A new subgenus, Menkeella subgen. nov., is proposed for S. paulinierii Guérin Méneville, 1843.

Four former synonyms are resurrected: Sphex pruinosus var. haemorrhoidalis Magretti, 1898, for which the replacement name of $S$. feijeni nom. nov. is proposed, $S$. camerunicus Strand, 1916, S. cinerascens Dahlbom, 1843 and S. abbotti W. Fox, 1891.

Nine of the previously valid names are shown to be junior synonyms: Sphex haemorrhoidalis basuto (Arnold, 1947) and S. haemorrhoidalis kobrowi (Arnold, 1928) = S. umtalicus Strand, 1916; S. incomptus anonymus Leclercq, $1955=$ S. nigrohirtus Kohl, 1895; S. neavei (Arnold, 1928) $=$ S. abbotti W. Fox, 1891; S. observabilis (R. Turner, 1918) = S. ahasverus Kohl, 1890; S. rufiscutis (R. Turner, 1918) and S. mochii Giordani Soika, $1942=$ S. jansei Cameron, 1910; S. rufiscutis laevigatus Arnold, $1951=$ S. gaullei Berland, 1927; and S. stadelmanni integer (Arnold, 1928) = S. stadelmanni Kohl, 1895. Three previously synonymized species are transferred as new synonyms to different species: Sphex conradti Berland, $1927=$ S. camerunicus Strand, 1916; S. kilimandjaroensis Cameron, $1908=$ S. abbotti W. Fox, 1891; and S. nigripes var. pachyderma Strand, $1916=$ S. umtalicus Strand, 1916. For three species, the actual type localities are on different continents than their labels indicate: Sphex ahasverus Kohl, 1890, S. castaneipes Dahlbom, 1843 and S. optimus F. Smith, 1856, with the first occuring in Africa instead of Australia and the other two presumably occuring in South America instead of Africa.

The occurrence of the argentatus group in Sub-Saharan Africa is confirmed, and seven additional species groups are established, with all but one of them based on apomorphic morphological traits. A hypothesis on the phylogenetic relationships among the subgenera and species groups of Sphex is presented, and an identification key for both sexes as well as a placoid-based identification table for males are presented. Using Bayesian inference and maximum likelihood analysis on mitochondrial and nuclear sequence data generated from thirty-one of the recognized taxa, the monophyly of the proposed species groups is corroborated.
\end{abstract}


Keywords. Sphex, Afrotropic, phylogeny, barcoding.

Dörfel T.H. \& Ohl M. 2022. The wasp genus Sphex in Sub-Saharan Africa (Hymenoptera: Sphecidae). European Journal of Taxonomy 796: 1-170. https://doi.org/10.5852/ejt.2022.796.1665

\section{Introduction}

Until now, there has been no comprehensive revision of all species of Sphex Linnaeus, 1758 from the Afrotropical region, and only few partial revisions and identification keys have been published. The most important ones are: Kohl 1895 (worldwide revision of the genus), Arnold 1928 (revision of species from southern Africa), Berland 1956 (revision of Afrotropical species) and Leclercq 1961 (key to haemorrhoidalis group). However, they are partly outdated and they contain numerous errors and omissions of important diagnostic characters. Prior to this publication, 31 species and nine additional subspecies were recognized for Sub-Saharan Africa (Pulawski 2020). Except for Sphex pruinosus Germar, 1817 and Sphex fumicatus Christ, 1791, of which the voucher specimens were collected in the Palearctic region (Schmid-Egger et al. 2018), no DNA sequences of Sphex from the Afrotropical region were previously available. An intrageneric phylogeny of Sphex has never been published before.

After studying large amounts of material and assessing the majority of published species descriptions of Sphex from tropical Africa, we attempt to provide a complete revision for the region. Furthermore, we use an integrative approach based on morphology as well as sequence data from the mitochondrial CO1 region and two nuclear genes in order to investigate the phylogenetic relationships between the species of the genus.

\section{Species treated}

In this publication, 42 species and four subspecies of African Sphex are recognized. Eleven species and all of the recognized subspecies were previously undescribed. They are native to the Afrotropical realm as defined by Udvardy (1975) or the region of southern Arabia and the islands of the Seychelles and Comoros, which are included here. Some species like Sphex pruinosus can also be found in the Palearctic.

We treat every valid species previously recorded from the Afrotropical region (Pulawski 2020). Two species are probably not of Afrotropical origin, namely S. castaneipes Dahlbom, 1843 and S. optimus F. Smith, 1856. The first was described based on a series allegedly collected at the Cape of Good Hope, the second based on a single individual supposedly from the Gambia. After having examined photographs of a syntype of $S$. castaneipes and the holotype of $S$. optimus, we conclude that these specimens were not actually collected in Africa, but possibly in the Neotropical region. They possess a markedly dense metanotal vestiture that completely obscures its surface, which is unknown in continental members of African Sphex, but present in several species from South America. Sphex optimus closely resembles S. melanopus Dahlbom, 1843 and could be synonymous with that species, although more thorough comparisons with Neotropical species are required to reach an informed decision. The status of $S$. castaneipes is dubious.

Many Afrotropical locality records of Sphex by previous authors were misidentifications. A list of all the corrections would be far too long, but any prior citation of material deposited in the IRSN, MRAC, NRS, OÖLM or ZMB (see list of abbreviations below) can be traced to the specimen list, as the African material of the genus present in these collections has been examined by us in its entirety. From the remaining collections, specimens with locality data already represented in the material list that belong to species which are very commonly found and which we classify as taxonomically unambiguous were not always included. 
To our knowledge, three species with alleged Afrotropical records presumably do not occur in this region. Thus, they will not be treated in detail. The first is $S$. argentatus Fabricius, 1787. Primarily found in the Oriental and Australian regions, an updated description was published in Dörfel \& Ohl (2015). Most African records are probably misidentifications of S. fumicatus, which is quite similar and occurs commonly almost everywhere in the region. We did come across two individuals of S. argentatus supposedly collected in the former Kasaï province of the Democratic Republic of the Congo, though they may be mislabeled. It seems doubtful that the species occurs in Africa.

Kohl (1906) described from the Arabian island of Socotra what he believed to be the previously unknown female of $S$. deplanatus Kohl, 1895, an Oriental species. After examining Kohl's specimens and comparing them with material of $S$. deplanatus, we repudiate his assumption. In the collection of the BMNH, there are not only more females but also males of this Socotran taxon, which we describe as $S$. socotrensis $\mathrm{sp}$. nov.

Finally, Dollfuss (2008) and several authors before him proposed that African records of S. praedator F. Smith, 1858, which were usually identified as its synonym S. luteipennis Mocsáry, 1883, pertain to a different, undescribed species. After having studied the holotype and several specimens of S. praedator, we agree with this assessment and describe them under the name $S$. pseudopraedator $\mathrm{sp}$. nov.

\section{Species groups}

In total, we divide the Afrotropical Sphex into eight species groups, seven of which are newly erected. The argentatus group was originally proposed by Hensen (1991) and previously mainly encompassed species from the Oriental and Australian regions. African members are distinguished by the same character combination as those from other regions.

The concept of the haemorrhoidalis group (occasionally called the haemorrhoidalis-bohemanni group), which was proposed by Arnold (1928) and treated in greater detail by Leclercq (1961), neglects important morphological characters. The group is clearly paraphyletic and is here replaced by an alternative group concept.

Nonetheless, we have chosen $S$. bohemanni Dahlbom, 1845 as the eponym for a new group because the species is easily recognizable and, unlike some other members of its group, does not have any synonyms widely-used in place of its actual valid name.

\section{Subspecies concepts and criteria}

Previous authors working with Afrotropical Sphex have often neglected to explain their reasoning when classifying taxa as subspecies. Indeed, Mayr (1942) stated that " $[\ldots]$ in the absence of definite criteria it is, in many cases, equally justifiable to consider certain isolated forms as subspecies or as species, to consider a variable species monotypic or to subdivide it into two or more geographical races, to consider well-characterized forms as subspecies of a polytypic species or to call them representative species." The final judgements are made by the taxonomists themselves, and most publications fail to provide standardized criteria for determining subspecific taxa (Haig et al. 2006).

Naturally, defining what consitutes a subspecies is directly tied to a species concept. One of the most well-known is the biological species concept (Mayr 1942), of which the central assumption is that populations of an animal species have the potential to interbreed with each other, but are reproductively isolated from populations of other species. While this definition places special emphasis on the importance of sexual reproduction, which is a key factor for speciation (Bernstein et al. 1985), a major difficulty regarding its practical application for taxonomists is the inability to directly observe and gather data on these interactions or lack thereof, at least in the majority of invertebrates. This is especially 
problematic when dealing with allopatric populations, as their ability or inability to interbreed with each other exists only in theory.

The issue can be tackled by establishing a unified species concept, which acknowledges species as separately evolving metapopulation lineages (De Queiroz 2007). Contingent upon the decrease in gene flow between these lineages, secondary characters like morphological and genetic distinctiveness arise. Once the speciation has progressed far enough, these indirect indicators can be used as criteria. Regarding morphology, an essential property of subspecies and one of the main reasons that warrant their description as separate taxa instead of individual varieties is the presence of at least one fixed diagnosable character state in a population that differs from the state found in other populations of the same species (Mayr 1982; Braby et al. 2012). Attempts have been made to also provide definitions for genetic standards. For example, distance-based clustering with a threshold of 3\% regarding CO1 sequences yields the most accurate results when comparing morphospecies in beetles (Astrin et al. 2012). Still, there is no indication that this specific value is universal among insects. The delimitation of criteria specific to the Afrotropical Sphex and their application is treated in more detail in the discussion.

\section{Unanswered questions}

There remain several issues that need to be addressed in future studies. First and foremost, while six of the eight species groups that contain members in the Afrotropical region have been classified as well-supported pairs of sister taxa, the relationship between these as well as their affiliation with the two remaining groups is still mostly unclear. As useful morphological characters are limited, the most reliable way to solve this problem will be the generation of additional sequence data. A better taxon sampling would certainly be helpful, but the primary issue is the lack of coverage within the genomes of the sequenced species, as the methods we used are quite unreliable for museum material. Newer techniques like target enrichment of ultraconserved elements (UCEs) are more suited to analyzing specimens with fragmented DNA and recovering generic phylogenies, as the number of loci is also much greater (Faircloth et al. 2014; Blaimer et al. 2016).

Finally, Sphex needs to be revised in other biogeographic realms as well, especially the Oriental and Neotropical regions. As species groups were shown to span several continents in their distribution, there are doubtlessly other species in the world that belong to the newly erected groups. Their discovery will aid our understanding of the phylogenetic history of the genus.

\section{Morphology}

The morphological characters used in this paper can be divided into three main groups:

a. coloration of the integument

b. coloration and other properties of the setae

c. surface structures

In the African Sphex, the coloration of the integument (except for the wings) ranges between black and ferruginous. We found evidence that it is subject to climatic and geographic influences and generally shows a higher intraspecific variability than other structural characters. For example, males of S. umtalicus Strand, 1916 from southern Africa usually have the mandibular base black and a dark ferruginous stripe in the center of the mandible, whereas some individuals from eastern Africa have the basal half of the mandible bright ferruginous. Although coloration is a valuable diagnostic in some species, structural characters are preferred in the keys.

Setal characters are very important as diagnostics both at the species and the species group level. In particular, the orientation of the erect setae on the propodeal enclosure, which has been neglected in 
previous publications, has been shown to be of high taxonomic significance. The setae face anteriorly, posteriorly, or perpendicularly to the propodeal surface.

Density and texture of the vestiture, particularly on the scutellum, is also an important diagnostic feature. In some cases, assessing density can be difficult, as many of the setae may have been lost due to abrasion, in which case only impressed regions of the integument might show the original condition. It must be determined whether a potential lack of vestiture is a result of abrasion, for example by checking the state of the wing apices or the mandibles of the specimen in question. Presumably because of their burrowing activity, female Sphex are much more affected by aging than males, although this can vary depending on species group or other, as yet unknown factors.

The coloration of the setae seems to be less subject to intraspecific variation than the color of the integument, although taxa which we, in concordance with previous authors, recognize as different subspecies are commonly distinguished only through the color of the vestiture on a single body region. Furthermore, some females can only be identified based on the luster of their appressed facial setae. This is visible in the vestiture on the lateral clypeal margins and on the paraocular area. At first glance, it looks primarily brown, black or transparent in some species, so these areas should be viewed from several different angles to determine whether the sheen of the setae has a different color, such as silvery, golden or ferruginous.

\section{Overview of character states}

The clypeal margin of female African Sphex often has at least one small medial notch, frequently three. A protruding tooth is only found in S. cinerascens Dahlbom, 1843. There can be a step-like structure above the free margin, or the transition to the convex main part of the clypeus can be gradual. Some males have a protruding tooth, and the free margin can be simple or subtly folded medially. Appressed setae may cover the entire clypeus, a central longitudinal line or parts of the lower half can be glabrous. In females, except of $S$. paulinierii Guérin-Méneville, 1843, the labrum is carinate.

The scutellum is convex or flat and can have a medial longitudinal impression in case of the former, though that seems to be intraspecifically variable. Scutellar vestiture can be coarse or fine and rarely nearly absent, not counting loss due to abrasion. The metanotum can be flat or raised and may be slightly or conspicuously bituberculate in the latter case.

The area anterior of the spiracle is often flat, though in some groups there is a swelling followed by a depression, which can give the upper metapleuron a concave appearance instead of a plane one.

As stated above, the erect propodeal setae are usually curved and can be oriented anteriorly, posteriorly or perpendicularly. The sculpture of the propodeal enclosure may be finely or more coarsely rugose, consisting of fine transversal lines or more or less distinct transversal ridges of varying number.

The male sternum can have fringes of long erect setae on sterna II-VII. Sternum VIII is densely but shortly pubescent. The posterior margin of sternum VII is sometimes notched, and the visible portion of sternum VIII may be triangular, rounded or emarginate. The penis valvae are partially or completely fused (the extent of fusion is not always constant within species), and the valvicepes are rounded and curved ventrally in their presumed unmodified state, but can be of various shapes.

Sexual dimorphism occurs in all species of Sphex. The most notable structures are mouthparts, clypeus, antenna, tarsal rake, mesosomal dorsum and apical metasomal segments, including genitalia.

As in the majority of Aculeata, male Sphex have 11 antennal flagellomeres, whereas females have 10. Placoids are sensory-cell-containing structures and are only found on the inner side of the male antenna 
between flagellomeres I and VII. They can be extremely useful for species identification. Length, width and position of placoids can be a crucial recognition character to determine species groups. Nonetheless, placoid shape and number are subject to intraspecific variation, so they are sometimes unreliable characters.

Another sexually dimorphic body part is the mandible. In males, the mandible is much more slender and has a simple tooth on the inner margin. In the female, the mandible is much longer and broader, and there is a small second inner tooth just proximally of the first one. These dissimilarities are most likely the result of their differences in behavior, because only females use the mandibles for prey manipulation and nest construction. In males, no carina is present on the labrum.

The clypeus in male Sphex from Africa is narrower than in females, has the free margin concave in most species and nearly always covered with appressed silvery setae, regardless of the color of the vestiture in the female. The clypeus can be covered entirely by the appressed setae, or there can be a glabrous spot in the lower center. If present, tooth-like processes do not emerge from the visible outer side of the free margin, as it is the case in females, but rather from the inner one (which is near the labrum). In females, the free clypeal margin is slightly convex, the coloration of the clypeal setae varies, and on the lower half of the clypeus itself there can be a more or less distinct indentation, whereas on the upper half a small longitudinal carina may be present. In males, both of these structures are usually absent or barely visible, even when present in the respective female, except in the nigrohirtus group.

In contrast to males, female Sphex possess long spines on the outer side of the foretarsomeres. They are used for digging out the nest, and their relative length can be a useful diagnostic for identifying some species groups.

Scutellum and metanotum are generally modified in a similar way regarding surface convexity. The scutellum and metanotum of the female are usually slightly flatter than in the respective male. The color of the propodeal setae is not always identical in conspecific males and females, and the same applies to the prominence of potential transversal ridges on the propodeal enclosure.

Finally, males of many species have important diagnostic characters on the apical two sterna of the metasoma and on the genital apparatus, like the presence or absence of dense setal fringes, the shape of posterior sternal margins or the properties of the dorsal side of the inner paramera. However, while genital morphology differs markedly in some groups of African Sphex, the majority of them do not show significant differences in male genitalia. Interestingly though, species from other regions can be quite diverse in the shape of their penis valvae, like the Nearctic Sphex (Bohart \& Menke 1963).

Females have no comparable attributes near the metasomal apex and are usually more difficult to identify, not least due to the lack of placoids.

\section{Biology}

Sphex construct ground nests that are usually multicellular and vary in depth between 3 and $75 \mathrm{~cm}$ depending on species and other factors (Bohart \& Menke 1976). As the female basitarsal rake is used for nest digging, the species-specific length and width of its spines is presumably an adaptation to the respective soil type. Sphex provision their larvae mainly with tettigoniids, occasionally also with gryllacridids. Nothing has been published so far on the specific nesting habits of any Afrotropical species, but we present a short list of Sphex that were observed or collected together with prey (Table 1).

Adults often visit flowers (Gess \& Gess 2003). 
Table 1. Known prey of Afrotropical Sphex Linnaeus, 1758, based on orthopterans collected in tandem with identified specimens and photographic observations (marked with asterisks) found at http://www.waspweb.org/Apoidea/Sphecidae/Sphecinae/Sphecini/Sphex/Sphex_taschenbergi.htm and https://www.inaturalist.org/observations/38705908.

\begin{tabular}{cc}
\hline Sphex species & Prey taxon \\
\hline S. ahasverus Kohl, 1890 & Gryllacrididae \\
S. torridus F. Smith, 1873 & Tylopsis bilineolata (Serville, 1838) (Phaneropterinae) \\
S. meridionalis (Arnold, 1947) & Ruspolia sp. (Conocephalinae) \\
S. taschenbergi Magretti, 1884* & Terpnistria sp. (Phaneropterinae) \\
S. tomentosus Fabricius, $1787^{*}$ & Terpnistria sp. (Phaneropterinae) \\
\hline
\end{tabular}

\section{Material and methods}

\section{Sources of material}

The material used in this study comes from the following institutions (names of collection heads or managers who arranged loans are given in parentheses). Abbreviations of institution names mostly follow Pulawski (2020). All individuals are dried and pinned.

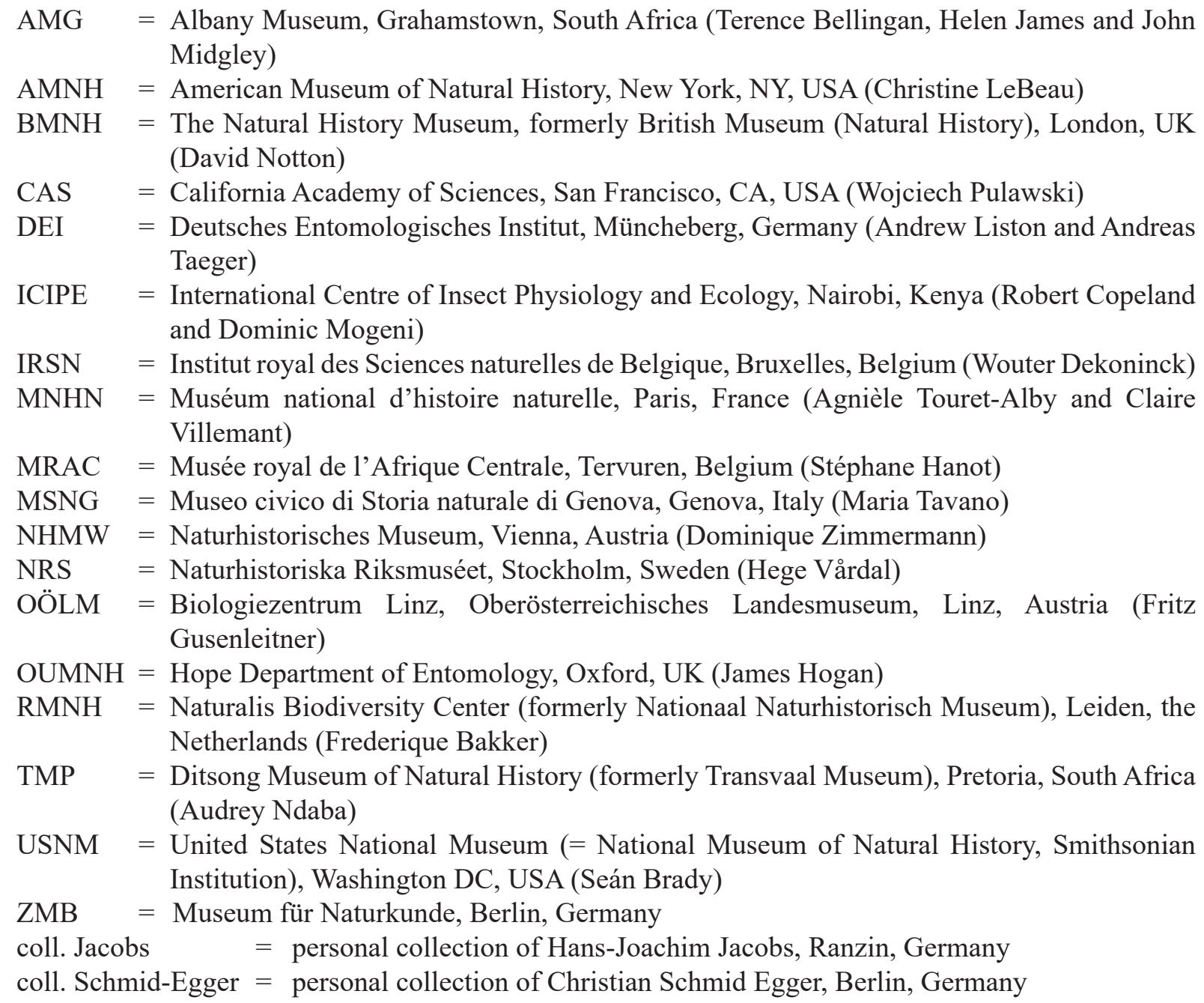


The following are depositories of types that are mentioned in the species descriptions but have not been examined personally for this study, though some of them have provided photographs.

ANSP = Academy of Natural Sciences, Philadelphia, PA, USA (Jason Weintraub)

HALLE $=$ Zoologisches Institut der Martin-Luther-Universität, Halle, Germany

MZLU = Lund University Biological Museum (formerly Lund Museum of Zoology), Lund, Sweden

SAM = South African Museum, Cape Town, South Africa

TMB = Természettudományi Múzeum, Budapest, Hungary

ZMUC = Zoological Museum(now Natural History Museum ofDenmark), University of Copenhagen, Copenhagen, Denmark

In total, we have examined over 3800 pinned specimens of Afrotropical Sphex, originally collected in 44 countries. Those with information precise enough for determining coordinates are mapped in Fig. 129.

A Leica S8 APO stereoscopic microscope and a Leica KL $1500 \mathrm{H}$ light source were used for optical examination. The measurements were done with an ocular micrometer.

Multi-focus photographs of specimens were taken with a Canon EOS 6D Mark II digital camera and a Canon Macro Ring Lite MR-14EX II flash device mounted on a Cognisys StackShot Macro Rail Set, and assembled using Helicon Remote ver. 3.9.9 W and Helicon Focus Pro ver. 7.

Images were edited and cleaned using Adobe Photoshop CS5.1 ver. 12.1 and arranged with Adobe Illustrator CS5.1, ver. 15.1.0.

\section{Molecular methods}

We follow Field et al. (2011) in selecting mitochondrial cytochrome oxidase I (CO1), nuclear elongation factor-1 $\alpha(E F-1 \alpha)$ and nuclear long wavelength rhodopsin (LWR) for phylogenetic reconstruction.

The unspecific primer pairs LCO1490-For and H7005-Rev, LWRhFor and LWRhRev, and HaF2For1 and F2-rev1 were sufficient to amplify the target genes from the Nearctic species Sphex ashmeadi (Fernald, 1906) and S. lucae de Saussure, 1867 which were collected four months prior and preserved in $96 \%$ ethanol. However, our tests have shown that these primers were insufficient for amplifying DNA from pinned specimens even when they were collected less than ten years before, probably due to DNA degradation. Therefore, we designed primer pairs for amplifying the respective genes by targeting regions which encompass one half or a quarter of the complete sequence at a time.

In this study, all African specimens from which gene regions have been successfully sequenced were collected between 1984 and 2013. This resulted in a lack of viable material for species which appear to be rare or that occur in regions which have not been the target of collecting trips in the more recent past.

Presumably because of advanced age and suboptimal conservation status for molecular analyses of most of the available material, only approximately $50 \%$ of the individuals in which an extraction was performed actually yielded usable DNA. Amplification of the selected regions proved to be difficult in most cases. For most samples that allowed amplification of the mitochondrial CO1 gene, attempts to amplify the nuclear genes LWR and EF-1 $\alpha$ were unsuccessful, and vice versa. Out of the overall 30 African taxa that yielded usable sequence data, there are merely ten for which we have data from regions of all three of the target genes, and for 12 we were only able to sequence regions from one of the genes (Table 2).

DNA extraction was done either by cutting open the thorax and removing the interior muscle tissue, or by removing one leg or tarsus, usually a hindleg, and then cutting it into very small pieces inside a reaction tube. Afterwards, the sample was processed using the Qiagen DNeasy ${ }^{\circledR}$ Blood \& Tissue Kit 
or the Qiagen QIAamp ${ }^{\circledR}$ DNA Investigator Kit in accordance with the respective protocol. Regarding isolation success, no significant differences between the two commercial products were observed.

DNA amplification was done using polymerase chain reaction (PCR) and a SensoQuest Labcycler Gradient, in volumes of $25 \mu 1$ with the following composition:

- $17.8 \mu 1$ purified $\mathrm{H} 2 \mathrm{O}$

- $2.5 \mu 110 \times$ reaction buffer

- $1 \mu \mathrm{MgCl} 2$

- $0.5 \mu 1 \mathrm{dNTPs}$

- $0.2 \mu \mathrm{Taq}$

- $1 \mu 1 \mathrm{~F}$-primer

- $1 \mu 1 \mathrm{R}$-primer

- $1 \mu 1$ sample DNA

PCR conditions were as follows: $95^{\circ} \mathrm{C}, 2 \mathrm{~min} ;\left[95^{\circ} \mathrm{C}, 1 \mathrm{~min}\right.$; Ta $\left.1 \mathrm{~min} ; 72^{\circ} \mathrm{C}, 1 \mathrm{~min}\right] \times 40$ cycles.

Primer synthesis was conducted by Metabion International AG. The following primer pairs were used:

EF-1 $\alpha$ :

- HaF2For1 (5'-GGGYAAAGGWTCCTTCAARTATGC-3') and F2-Rev1 (5'-AATCAGCAGCACCTTTAGGTGG-3'), Ta $=54^{\circ} \mathrm{C}$ (Danforth et al. 1999)

- EF1alpha-mini-For (5'-TAAGGAAGGCAAGGCTGAAGG-3') and EF1alpha-mini-Rev ( $5^{\prime}$ - CGGTACCGATACCACCGATT-3'), Ta $=58^{\circ} \mathrm{C}, 338$ bp product

LWR:

- LWRhFor (5'-AATTGCTATTAYGARACNTGGGT-3') and LWRhRev (5'-ATATGGAGTCCANGCCATRAACCA-3'), Ta $=52^{\circ} \mathrm{C}$ (Danforth et al. 2004)

- LWR-1 sthalf-For (5'-ATCTACGCGATGTTGGGCTC-3') and LWR-1 sthalf-Rev ( $5^{\prime}$ - TCCCTGCTGAAGTAGTCGGT- $\left.3^{\prime}\right), \mathrm{Ta}=58^{\circ} \mathrm{C}, 345 \mathrm{bp}$ product

- LWR-2ndhalf-For (5'-CCCGAGGGTAACATGACCG-3') and LWR-2ndhalf-Rev ( $5^{\prime}$ - GGAGATGGTCATCAGGGCAA- $\left.3^{\prime}\right), \mathrm{Ta}=58^{\circ} \mathrm{C}, 352 \mathrm{bp}$ product

- LWR-quar1-F (5'-ATCTACGCGATGTTGGGCTC-3') and LWR-quar1-R (5'- GGGAGAAGAGCCAGATGCC-3'), Ta $=51.3^{\circ} \mathrm{C}, 160$ bp product

- LWR-quar2-F (5'-AAATCCTGGGCATCTGGCTC-3') and LWR-quar2-R (5'- CCCTGCTGAAGTAGTCGGTG-3'), Ta $=58.7^{\circ} \mathrm{C}, 202$ bp product

CO1:

- LCO1490-For (5'-GGTCAACAAATCATAAAGATATTGG-3') and H7005-Rev (5'-ACNACRTARTANGTRTCRTG-3'), Ta $=52^{\circ} \mathrm{C}$ (Folmer et al. 1994; Hafner et al. 1994)

- Sphex-MinibarF1 (5'-GGAACAGGTACAGGAACAGGA-3') and Sphex-MinibarR1 (5'-TCTCCTCCTCCGATAGGATCAA-3'), Ta $=52.7^{\circ} \mathrm{C}, 326$ bp product

- CO1-start-For (5'-AGAATGTGAGCAGGAATAATAGGAT-3') and CO1-start-Rev (5'- TCAACCAGTTCCTGTTCCTGT-3'), Ta $=47.7^{\circ} \mathrm{C}, 312$ bp product

- CO1-quar1-F (5'-TGAGCTGGAATAATAGGATCATCTC-3') and CO1-quar1-R (5'- ACTAATCAATTTCCAAATCCTCCA-3'), Ta $=45.9^{\circ} \mathrm{C}, 176 \mathrm{bp}$ product

- CO1-quar2-F (5'-ATTGGAGGATTTGGAAATTGAT-3') and CO1-quar2-R (5'- AAAATAATATTAGATAGGGGTGGA-3'), $\mathrm{Ta}=45^{\circ} \mathrm{C}, 188 \mathrm{bp}$ product 
Table 2. For each African taxon where DNA sequencing was successful, the total length of the recovered sequences is listed. Data obtained from BOLD or GenBank are marked with 'ext.' (external). Where sequences were obtained from multiple specimens, the respective gene region and voucher ID are represented by matching colors. In one case, $\mathrm{CO} 1$ sequence fragments from two specimens were combined into a single longer one (bicolored cell). Further data on each voucher specimen are listed in Supplementary file 1. The names of new species or subspecies are highlighted in bold.

\begin{tabular}{|c|c|c|c|c|c|}
\hline Taxon & Accession numbers & Voucher ID & CO1 & EF-1 $\alpha$ & LWR \\
\hline abbotti abbotti & MW538552; MW582289 & THD-032-ICIPE & $168 \mathrm{bp}$ & - & $560 \mathrm{bp}$ \\
\hline abbotii nivarius & MW538559 & THD-016-OOLM & $316 \mathrm{bp}$ & - & - \\
\hline abyssinicus & MW582276 & THD-033-OOLM & - & - & $560 \mathrm{bp}$ \\
\hline pseudopraedator & MW538560; MW582290 & $\begin{array}{l}\text { THD-000-ZMB + } \\
\text { THD-027-ZMB }\end{array}$ & $342 \mathrm{bp}$ & - & $573 \mathrm{bp}$ \\
\hline $\begin{array}{l}\text { schoutedeni } \\
\text { malawicus }\end{array}$ & MW538575; MW582287 & THD-028-ZMB & $593 \mathrm{bp}$ & - & $190 \mathrm{bp}$ \\
\hline $\begin{array}{l}\text { stadelmanni } \\
\text { stadelmanni }\end{array}$ & MW538556; MW582288 & $\begin{array}{c}\text { THD-008-ZMB + } \\
\text { THD-009-ZMB }\end{array}$ & $265 \mathrm{bp}$ & - & $542 \mathrm{bp}$ \\
\hline stadelmanni rufus & MW538562 & THD-014-OOLM & $327 \mathrm{bp}$ & - & - \\
\hline ahasverus & MW538553; MW582291 & THD-011-ZMB & $224 \mathrm{bp}$ & - & $673 \mathrm{bp}$ \\
\hline incomptus & MW582292 & THD-035-OOLM & $101 \mathrm{bp}$ & - & $326 \mathrm{bp}$ \\
\hline nigrohirtus & MW538567; MW582293 & THD-013-OOLM & $458 \mathrm{bp}$ & - & $192 \mathrm{bp}$ \\
\hline camerunicus & MW538563 & THD-023-OOLM & $329 \mathrm{bp}$ & - & - \\
\hline erythrinus & MW538558; MW558242; MW582282 & THD-024-CAS & $326 \mathrm{bp}$ & $338 \mathrm{bp}$ & $628 \mathrm{bp}$ \\
\hline fumicatus & MW538580; MW558245 & $\begin{array}{c}\text { GenBank: MH608968.1 } \\
\text { / THD-007-ZMB }\end{array}$ & ext. & $320 \mathrm{bp}$ & - \\
\hline lanatus & MW538579; MW558243; MW582281 & $\begin{array}{l}\text { THD-005-ZMB + } \\
\text { THD-021-OOLM }\end{array}$ & $613 \mathrm{bp}$ & $341 \mathrm{bp}$ & $604 \mathrm{bp}$ \\
\hline taschenbergi & MW538576; MW558244; MW582283 & THD-004-ZMB & $589 \mathrm{bp}$ & $153 \mathrm{bp}$ & $627 \mathrm{bp}$ \\
\hline tomentosus & MW538551; MW558239; MW582284 & THD-006-ZMB & $165 \mathrm{bp}$ & $162 \mathrm{bp}$ & $647 \mathrm{bp}$ \\
\hline torridus & MW538577 & THD-017-OOLM & $607 \mathrm{bp}$ & - & - \\
\hline decipiens & MW538566; MW558235; MW582286 & $\begin{array}{c}\text { THD-001-ZMB + } \\
\text { THD-010-ZMB }\end{array}$ & $445 \mathrm{bp}$ & $240 \mathrm{bp}$ & $533 \mathrm{bp}$ \\
\hline jansei & MW538561 & THD-025-ZMB & $325 \mathrm{bp}$ & - & - \\
\hline pruinosus & MW538578; MW558236; MW582275 & $\begin{array}{c}\text { GenBank: MH610705.1 } \\
\text { / THD-036-ZMB }\end{array}$ & ext. & $281 \mathrm{bp}$ & $534 \mathrm{bp}$ \\
\hline schmideggeri & - & $\begin{array}{l}\text { BOLD: BC-ZSM- } \\
\text { HYM-30269-D07 }\end{array}$ & ext. & - & - \\
\hline pseudosatanas & MW538569; MW558238; MW582278 & THD-029-BMNH & $511 \mathrm{bp}$ & $358 \mathrm{bp}$ & $681 \mathrm{bp}$ \\
\hline rufoclypeatus & MW538570 & THD-012-OOLM & $552 \mathrm{bp}$ & - & - \\
\hline satanas & MW538568 & THD-018-OOLM & $524 \mathrm{bp}$ & - & - \\
\hline haemorrhoidalis & MW538572; MW538573 & $\begin{array}{l}\text { THD-020-OOLM + } \\
\text { THD-034-OOLM }\end{array}$ & $606 \mathrm{bp}$ & - & - \\
\hline mweruenis & MW538574 & THD-019-OOLM & $592 \mathrm{bp}$ & - & - \\
\hline umtalicus & MW538571; MW558237; MW582277 & THD-002-ZMB & $585 \mathrm{bp}$ & $334 \mathrm{bp}$ & $366 \mathrm{bp}$ \\
\hline meridionalis & MW538564; MW558240; MW582279 & $\begin{array}{c}\text { THD-003-ZMB + } \\
\text { THD-026-ZMB }\end{array}$ & $445 \mathrm{bp}$ & $337 \mathrm{bp}$ & $646 \mathrm{bp}$ \\
\hline nefrens & MW538565; MW558241; MW582280 & THD-015-OOLM & $451 \mathrm{bp}$ & $341 \mathrm{bp}$ & $646 \mathrm{bp}$ \\
\hline comorensis & MW538557 & THD-030-MRAC & $316 \mathrm{bp}$ & - & - \\
\hline malagassus & MW538555; MW582285 & THD-022-RMNH & $283 \mathrm{bp}$ & - & $192 \mathrm{bp}$ \\
\hline cinerascens & MW538554 & THD-031-OOLM & $271 \mathrm{bp}$ & - & - \\
\hline
\end{tabular}


New primers were designed with the help of NCBI Primer-BLAST (https://www.ncbi.nlm.nih.gov/tools/primer-blast/) based on sequence data from Sphex ichneumoneus Linnaeus, 1758, accession numbers JF927357.1 (CO1), JF927399.1 (LWR) and JF927441.1 (EF-

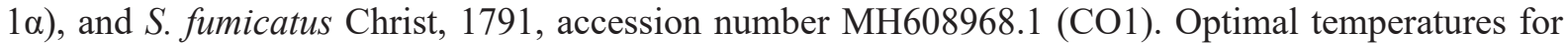
annealing of these primers were determined through gradient PCRs.

PCR success was assessed on a 1\% agarose gel using GelRed for staining and a Syngene G:BOX F3 with the software GeneSys ver. 1.6.9.0 for visualization. Sequencing was done by Macrogen, Inc.

We noticed that amplification of target regions was often less successful in members of the bohemanni group compared to individuals of other species, even when the specimens had been collected on the same day. Presumably, the reason for this is the larger body size and stouter build that characterize these species. One would have assumed that this might result in a greater amount of tissue being recoverable from the specimen and thus increase the chance of yielding intact DNA for a higher success rate of the PCR. However, it seems any positive effect which may have resulted from this factor was more than compensated by other negative influences. Since it is very likely that all specimens we examined were air-dried, we theorize that the greater mass and volume of the insect prolonged the time it took until all of the water (naturally contained in the body or as a component of the killing agent that was used) had evaporated from the body. As a result, DNA degradation processes, which are known to occur in the presence of free water (Lindahl 1993), continued for a longer period in these specimens than in smaller ones.

\section{Phylogenetic analysis}

Sequence data were edited and joined using BioEdit ver. 7.1.3.0 (Hall 1999) and aligned with each other using Clustal Omega (https://www.ebi.ac.uk/Tools/msa/clustalo/). The data were combined into a single dataset using Sequence Matrix ver. 1.8 (http://www.ggvaidya.com/taxondna/) and partitioned accordantly to the three gene regions, which was reassessed using PartitionFinder ver. 2.2.1 (http://www.robertlanfear.com/partitionfinder/). Two separate analyses were conducted on the molecular data.

For Bayesian inference (BI) analysis, the substitution model, suitable for each gene partition, was calculated using PAUP* ver. 4.0a166 (Swofford 2003) and MrModeltest ver. 2.4 (Nylander 2004). The following models were calculated to be ideal:

$\mathrm{CO} 1: \mathrm{GTR}+\mathrm{I}+\mathrm{G} 4$

LWR: $\mathrm{HKY}+\mathrm{F}+\mathrm{G} 4$

$\mathrm{EF}-1 \alpha:$ TIM2e+G4

GTR stands for general time-reversible model (Tavaré 1986), which assumes unequal rates and unequal base frequencies, and the parameter combination $+\mathrm{I}$ and $+\mathrm{G}$ allows for a proportion of invariable DNA sites and varying rates across these according to a discrete gamma distribution with four rate categories (Yang 1994; Gu et al. 1995). HKY (Hasegawa et al. 1985) is a substitution model with unequal transition/ transversion rates and unequal base frequencies, and the parameter $+F$ results in an empirical assessment of base frequencies from the data. Finally, TIM2e is a transitional model with equal base frequencies (Posada \& Crandall 1998).

BI analysis using 4 chains was conducted by setting generations to 4 million, trees were sampled every 100 generations, the first $25 \%$ generations were discarded as burn-in and posterior probabilities (PP) were then calculated from the posterior distribution of the retained Bayesian trees. 
For maximum likelihood (ML) analysis, the substitution model for each partition was calculated using ModelFinder (Kalyaanamoorthy et al. 2017) as part of the analysis with W-IQ-TREE (Trifinopoulos et al. 2016). The suggested models were identical.

\section{Maps and locality data}

All maps in this publication only contain data from specimens we examined personally, as potential misidentifications from previous records would otherwise distort the results. Exceptions are the type specimens of synonyms which we have not seen but can reasonably presume to be correctly identified. The maps were created with the help of SimpleMappr (https://www.simplemappr.net). Since coordinates had not been recorded for the majority of specimens examined, given localities in these cases were searched on www.geonames.org and in entomological or botanical locality lists (Bamps 1982; Bezdek et al. 2012). To retain discriminability of coordinates given on the label from those we added through retrospective research, the latter are given in square brackets in the material list. All localities which were deemed precise enough to reasonably pinpoint are present in the respective maps.

\section{Terminology}

Morphological terms largely follow Bohart \& Menke (1976). The term 'appressed setae' is used to distinguish the short setae from the longer erect ones on the same body area, even though in some cases the short setae may also be partially erect.

'Paraocular area' refers to the region between the dorsolateral clypeal margin and the compound eye.

Length ratios given in this paper are calculated as follows: for the petiole (Fig. 11), dorsal length is divided by medial width; for the tarsal rake (Fig. 12), the marked distance $\beta$ on the outer side of the foretibia is divided by the length of the secant that intersects the base and tip of the antepenultimate spine (arrow).

\section{Keys}

The first key is used to identify the species groups as well as individual species which are either incertae sedis or prone to be misplaced due to less conspicuous group-specific characters. The subsequent keys are for each of the eight species groups and contain all members, even if they were previously covered in the group key. Finally, we include a comparison table for male placoid configurations, as they often allow identification to at least group level (Table 3).

\section{Key to species groups and ungrouped species of Sphex Linnaeus, 1758}

1. Erect setae medially on propodeal enclosure extremely short $(\leq 0.2 \mathrm{~mm})$; fore- and hindwing yellow with fuscous margin, scutellum and propodeal enclosure ferruginous (Fig. 39). Body length greater than $25 \mathrm{~mm}$.........S. paulinierii Guérin-Méneville, 1843 (subgenus Menkeella subgen. nov.)

- Length of most erect setae medially on propodeal enclosure at least $0.5 \mathrm{~mm}$; color combination different

2. Metanotum markedly raised, usually with distinct median impression (Fig. 7). Area between metapleural sulcus and upper spiracular groove concave, with bulge anterior to the spiracular groove (Fig. 9) argentatus group (Key A)

- Metanotum not strongly raised, with indistinct impression or not impressed (Fig. 8), or area between metapleural sulcus and upper spiracular groove convex or plane and not bulging (Fig. 10) ${ }^{1}$

\footnotetext{
1 In several species groups, the metanotum is raised but has very indistinct tubercles and no bulging near the spiracle is present. On the other hand, in the satanas group the bulging is present, but the metanotum is always flat.
} 
3. Erect propodeal setae oriented posteriorly (Fig. 1) …..................................................................

- Erect propodeal setae oriented anteriorly or perpendicularly (Figs 2-4) .....................................5

4. Female: appressed setae on face uniformly silvery (Fig. 13) or rich golden. Male: cellular wing area without yellow tinge; sternum VIII posterolaterally concavely emarginate

nigrohirtus group (Key B)

- Female: appressed setae on clypeus at least medially dark brown (Figs 14-18). Male: apical part of sternum VIII semicircular (Fig. 20) or rectangular (Fig. 25), if concavely emarginate then cellular wing area with conspicuous yellow tinge (Fig. 93) bohemanni group (Key C)

5. Petiole length more than $3 \times$ its medial width; basal half of forewing with yellow tinge and yellowish veins (Fig. 38)

- Petiole much shorter, its length at most $2.8 \times$ its medial width. If longer, then wings without yellow tinge and wing veins dark brown (Fig. 100)

6. Free clypeal margin in both sexes medially with distinct tooth-like process. Male: placoids on flagellomeres IV-VI, covering their entire length. Western Africa ......S. cinerascens Dahlbom, 1843

- Process at free clypeal margin broad and very short or absent. Male: placoids on flagellomeres V and VI, covering only their basal third. South and central Asia, Arabian Peninsula ......

S. obscurus Fabricius, 1804

7. Erect propodeal setae oriented anteriorly, especially laterally on propodeal enclosure (Fig. 2) ....8

- Erect propodeal setae oriented perpendicularly (Figs 3-4)

8. Female: scutellum and metanotum markedly flat (Fig. 5). Male: clypeus uniformly black or at most free margin ferruginous; erect clypeal setae silvery; petiole length less than $3 \times$ its medial width satanas group (Key D)

- Female: scutellum and metanotum convex (Fig. 6). Male: clypeus uniformly black or lower third or half ferruginous (Fig. 28); erect clypeal setae silvery, golden or black; petiole length in one species more than $3 \times$ its medial width (Fig. 100)

gaullei group (Key E)

9. Metanotum not raised medially, with silvery-golden vestiture (Figs 119-120). Longest erect setae in anterior center of propodeal enclosure at least $0.75 \mathrm{~mm}$. Madagascar and surrounding islands malagassus group (Key F)

- Metanotum raised medially, with black vestiture (Figs 3-4). Longest erect setae in anterior half of propodeal enclosure no more than $0.65 \mathrm{~mm}$. Continental Africa

10. Female: scutellar vestiture coarse and dense (Figs 3-4); usually some erect setae (similar length as the long erect metanotal setae) present near scutellar center; propodeal sculpture discontinuously rugose; outer side of midtibial apex with distinctly curved spine. Male: free clypeal margin medially with distinct tooth-like process (Fig. 27) .....

umtalicus group (Key G)

- Female: scutellar vestiture fine and less dense (Fig. 8); scutellar center without any long erect setae like the ones on the metanotum (with long erect setae only being present near posterolateral scutellar margin); propodeal sculpture with fine transverse continuous carinae; spines on outer side of midtibial apex straight. Male: free clypeal margin without tooth-like process (Fig. 28) ......11

11. Female: clypeus black except for ferruginous free margin (Fig. 101). Male: appressed facial setae silvery; free clypeal margin largely black, lower clypeal center sometims ferruginous; basal half of mandible black with only a ferruginous stripe medially on mandible (Fig. 28)

S. decipiens Kohl, 1895

- Female: clypeus completely ferruginous (Figs 36-37). Male: appressed facial setae brassy or golden; free clypeal margin and basal half of mandible largely ferruginous

meridionalis group $($ Key $\mathrm{H})$ 

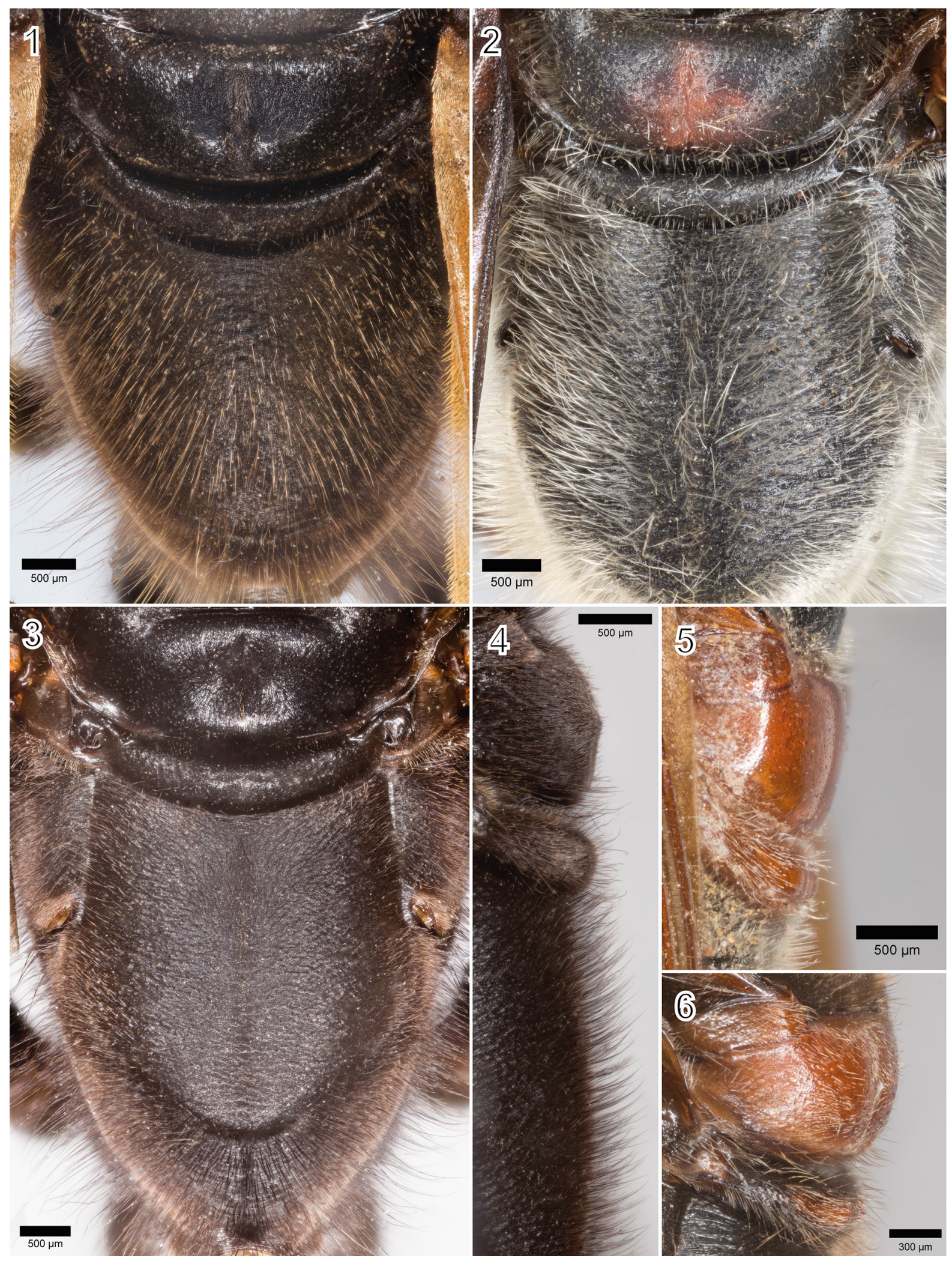

Figs 1-6. 1-3. Dorsal view of propodeal setae in different orientation. 4. Lateral view of thoracic dorsum. 5-6. Lateral view of scutellum and metanotum. 1. Sphex pseudopraedator sp. nov., $ᄋ$. 2. S. jansei Cameron, 1910, ô. 3-4. S. umtalicus Strand, 1916, ․ 5. S. rufoclypeatus sp. nov., ㅇ. 6. S. gaullei Berland, 1927, ․ . 


\section{Key A: Species of the Sphex argentatus group}

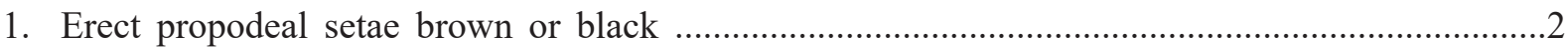

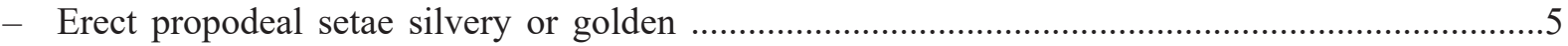

2. Cellular wing area with yellow or ferruginous tinge; wing base not notably infuscate (Figs 5859). Madagascar and surrounding islands only

- Cellular wing area without yellow tinge; wing base markedly fuscous (Figs 49, 52). Widespread

3. Female: apical margin of fore- and hindwing completely fuscous (Fig. 58). Male: erect clypeal and paraocular setae uniformly black

S. torridus F. Smith, 1873

- Female: apical margin of forewing only anteriorly with fuscous spot, apex of hindwing hyaline. Male: erect clypeal setae silvery intermixed with black ones; erect paraocular setae uniformly silvery-golden

S. voeltzkowii Kohl, 1909

4. Erect clypeal setae entirely or largely silvery (Fig. 53)

S. fumicatus Christ, 1791

- Erect clypeal setae black (Fig. 54) S. taschenbergi Magretti, 1884

5. Appressed and erect setae on face and propodeum rich golden, sometimes silvery. Hindfemur and most of hindtibia brightly ferruginous (Fig. 57)

S. tomentosus Fabricius, 1787

- Erect setae on face silvery or silvery-golden, sometimes intermixed with black ones; erect setae on propodeum silvery (Figs 41-44, 50-51). Hindleg darker

6. Erect clypeal setae at least partially black. Seychelles

S. rufinervis Pérez, 1895

- Erect clypeal setae uniformly silvery

7. Female: wings with yellow tinge (Fig. 41); petiole length less than $1.7 \times$ its medial width. Male: very broad placoids (nearly width of segment) covering flagellomeres III-VI

S. erythrinus (Guiglia, 1939)

- Female: wings without yellow tinge (Figs 43, 50); petiole length more than $1.7 \times$ its medial width. Male: placoid(s) more narrow (at most half as wide as segment), covering flagellomeres III-V or only V

8. Female: lower part of clypeus brightly ferruginous; propodeal enclosure not obscured by vestiture, with 7-10 distinct transversal ridges (Fig. 43). Male: setae laterally on gonostyles hyaline (Fig. 46); mid- and hindlegs completely black; placoids covering entire length of flagellomeres III-V

S. feijeni nom. nov.

- Female: clypeus black; propodeal enclosure without any distinct ridges, posterior half obscured by appressed setae (Fig. 50). Male: gonostyles laterally with stout black setae (compare Fig. 45); mid- and hindlegs partially ferruginous; single placoid on proximal half of flagellomere $\mathrm{V}$..

..S. lanatus Mocsáry, 1883

\section{Key B: Species of the Sphex nigrohirtus group}

1. Propodeal setae golden or silvery-golden, obscuring sculpture completely

S. ahasverus Kohl, 1890

- Propodeal setae black or silvery-white, leaving sculpture partially visible 

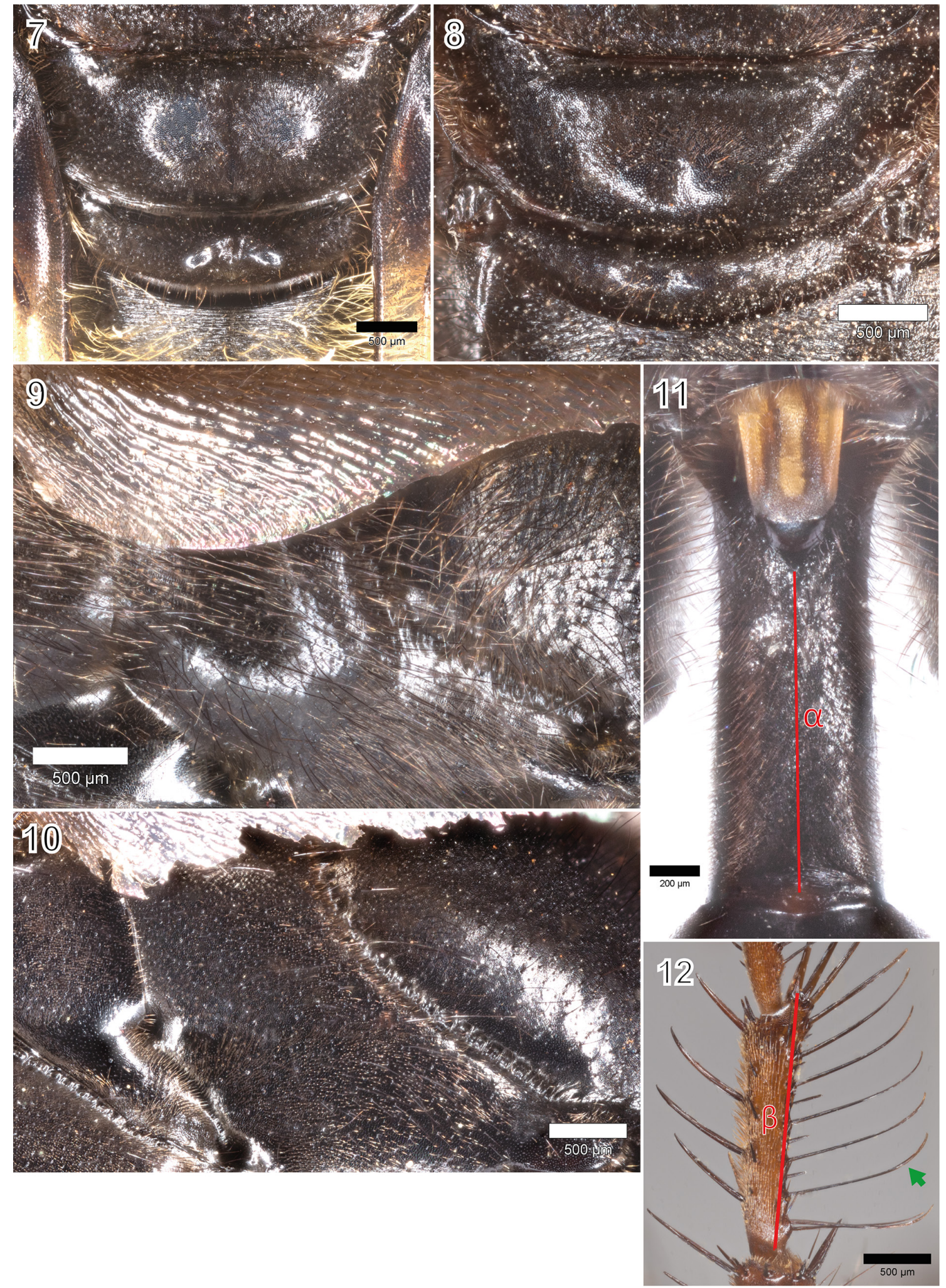

Figs 7-12. 7-8. Dorsal view of scutellum and metanotum. 9-10. Lateral view of upper metapleural area $($ anterior $=$ left). 11. Dorsal view of petiole. 12. Basitarsal rake. 7. Sphex tomentosus Fabricius, 1787, $\lambda$. 8. S. meridionalis (Arnold, 1947), ․ 9. S. torridus F. Smith, 1873, ô. 10. S. nigrohirtus Kohl, 1895, ㅇ. 11. S. haemorrhoidalis Fabricius, 1781, ․ 12. S. decipiens Kohl, 1895, ㅇ. $\alpha$ : defined as petiole length; $\beta$ : defined as length of outer side of tarsomere I; arrow: antepenultimate spine. 
2. Female: body length $>28 \mathrm{~mm}$; mandible black with only small dark ferruginous stripe centrally; conspicuous clypeal indentation. Male: flagellomeres IV-VII with broad placoids covering their entire length; apical part of penis valvae dorsolaterally with concave emargination (Figs 79-80)

S. camerunicus Strand, 1916

- Female: body length $<28 \mathrm{~mm}$; basal half of mandible bright ferruginous; smaller clypeal indentation. Male: flagellomere V or VI with narrow placoid; apical part of penis valvae dorsolaterally flat or convex (Figs 81-82)

3. Significant portion of erect setae on inner part of propodeal enclosure silvery (Fig. 85). Male: cellular wing area partially hyaline, with pale violet iridescence

S. incomptus Gerstaecker, 1871

- Erect setae on propodeal enclosure black, silvery near posterior margin at most (Fig. 86). Male: cellular wing area uniformly slightly fuscous, with conspicuous purple (Fig. 87) or cyan-violet iridescence

4. Wings with cyan iridescence. Female: appressed setae posterolaterally on collar and propodeum and erect setae on posterior propodeal margin uniformly black (Fig. 84)

S. nigrohirtus Kohl, 1895

- Wings without any cyan iridescence. Female: appressed setae posterolaterally on collar and propodeum and erect setae on posterior propodeal margin silvery (Fig. 86). Male: iridescence on forewing Byzantium-colored (Fig. 87)

S. pulawskii sp. nov.

\section{Key C: Species of the Sphex bohemanni group}

\section{Females}

1. Clypeus, trochanter, femora and tibiae largely ferruginous (Fig. 96)

- Clypeus and legs almost entirely black

S. stadelmanni rufus subsp. nov.

2. Cellular wing area with yellow tinge (Fig. 93)

S. pseudopraedator sp. nov.

- Cellular wing area more or less fuscous, without yellow tinge .3

3. Appressed clypeal and paraocular setae black, without differently-colored luster (Figs 15-16) ..

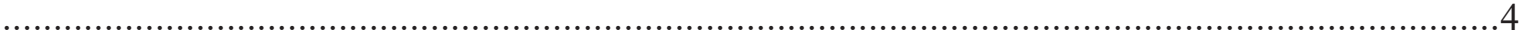

- Appressed lateroclypeal and paraocular setae with silvery, golden or ferruginous luster (Figs 14, $17-18)$

4. Free clypeal margin black; mandible black with dark ferruginous stripe only in center (Fig. 15) S. abbotti abbotti W. Fox, 1891

- Free clypeal margin and basal half of mandible dark ferruginous (Fig. 16)

..S. stadelmanni stadelmanni Kohl, 1895

5. Erect propodeal setae completely black

- Erect propodeal setae partially or entirely silvery

6. Free clypeal margin black; mandible black with dark ferruginous stripe only in center (Fig. 17) S. abyssinicus (Arnold, 1928)

- Free clypeal margin and basal half of mandible dark ferruginous (Fig. 18)

S. schoutedeni malawicus subsp. nov.

7. Petiole length about $2 \times$ its medial width

S. abbotti nivarius subsp. nov.

- Petiole length at most $1.7 \times$ its medial width S. schoutedeni schoutedeni Kohl, 1913 

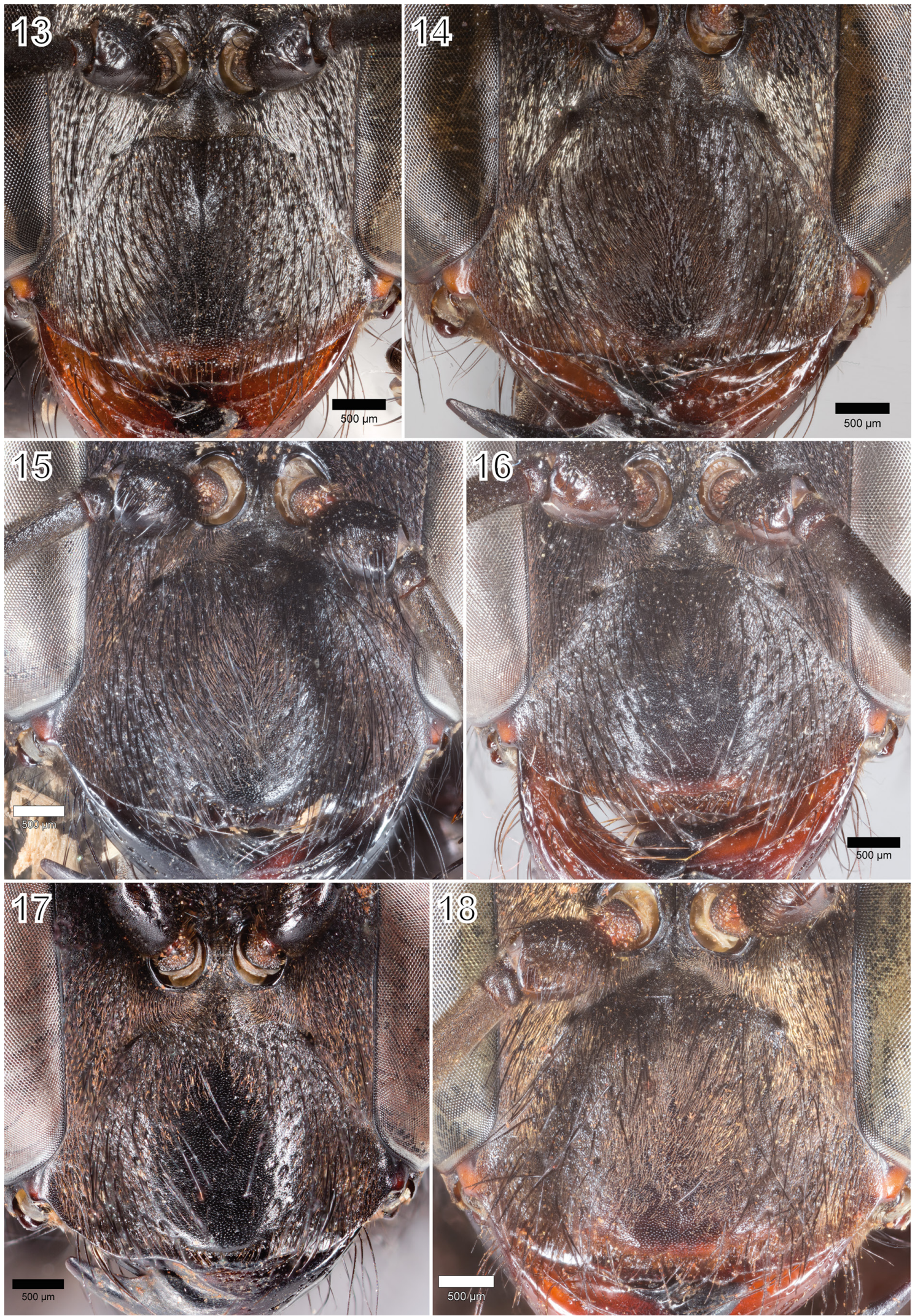

Figs 13-18. Faces of females in frontal view. 13. Sphex nigrohirtus Kohl, 1895. 14. Sphex abbotti nivarius subsp. nov. 15. S. abbotti abbotti W. Fox, 1891. 16. S. stadelmanni stadelmanni Kohl, 1895. 17. S. abyssinicus (Arnold, 1928). 18. S. schoutedeni malawicus subsp. nov. 


\section{Males}

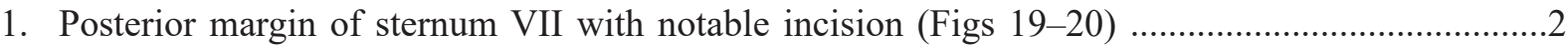

- Posterior margin of sternum VII without incision (Fig. 25) .......................................................

2. Depth of incision no more than half the visible part of sternum VII (Fig. 19)

S. bohemanni Dahlbom, 1845

- Depth of incision nearly as long as visible part of sternum VII (Fig. 20) .3

3. Appressed and erect propodeal setae completely black (Fig. 88) .....S. abbotti abbotti W. Fox, 1891

- Erect and sometimes appressed propodeal setae at least partially silvery (Fig. 89)

S. abbotti nivarius subsp. nov.

4. Posterior margin of sternum VIII rectangularly truncate (Fig. 25) .....S. abyssinicus (Arnold, 1928)

- Posterior margin of sternum VIII rounded or laterally concave

5. Cellular wing area with yellow tinge (Fig. 93)

S. pseudopraedator sp. nov.

- Cellular wing area more or less fuscous, without yellow tinge

6. Fused penis valvae laterally widened and entire; valviceps oriented obliquely (Figs 21-22) .........7

- Fused penis valvae laterally not widened but notched; valviceps oriented perpendicularly (Figs 2324)

7. Clypeus and legs mostly black (Fig. 95); appressed facial setae silvery-white

..S. stadelmanni stadelmanni Kohl, 1895

- Lower clypeal center, trochanters, femora and tibiae largely ferruginous (Fig. 97); appressed facial setae brassy S. stadelmanni rufus subsp. nov.

8. Erect propodeal setae at least partially silvery near center (Fig. 94)

S. schoutedeni schoutedeni Kohl, 1913

- Erect propodeal setae centrally black, golden at posterior margin

S. schoutedeni malawicus subsp. nov.

\section{Key D: Species of the Sphex satanas group}

1. Female: scutellum and metanotum completely black (Figs 113-114). Male: cellular wing area at least partially fuscous; narrow placoids on proximal halves of flagellomeres IV-VI; free clypeal margin medially with folding (compare Fig. 28)

- Female: scutellum at least on posterior half and metanotum ferruginous (Figs 109-110, 116). Male: cellular wing area hyaline; placoids broader and covering entire length of flagellomeres III-VI or free clypeal margin without folding

2. Female: propodeal setae silvery (Fig. 113)

S. satanas satanas Kohl, 1898

- Female: propodeal setae black (Fig. 114)

S. satanas memnon subsp. nov.

3. Female: anterior half of scutellum black (Fig. 116). Male: at least femora bright ferruginous (Fig. 117). Socotra ..S. socotrensis sp. nov.

- Female: scutellum entirely ferruginous (Figs 109-110). Male: legs completely black (Fig. 111). Mainland Africa

4. Female: upper half of clypeus as well as complete scutum black (Fig. 109). Male: free clypeal margin without folding; narrow placoids on proximal halves of flagellomeres IV-VI

S. pseudosatanas sp. nov.

- Female: all of clypeus and anterior half of scutum ferruginous (Fig. 110). Male: free clypeal margin medially with folding (compare Fig. 28); broad placoids covering entire length of flagellomeres III-VI

S. rufoclypeatus sp. nov. 

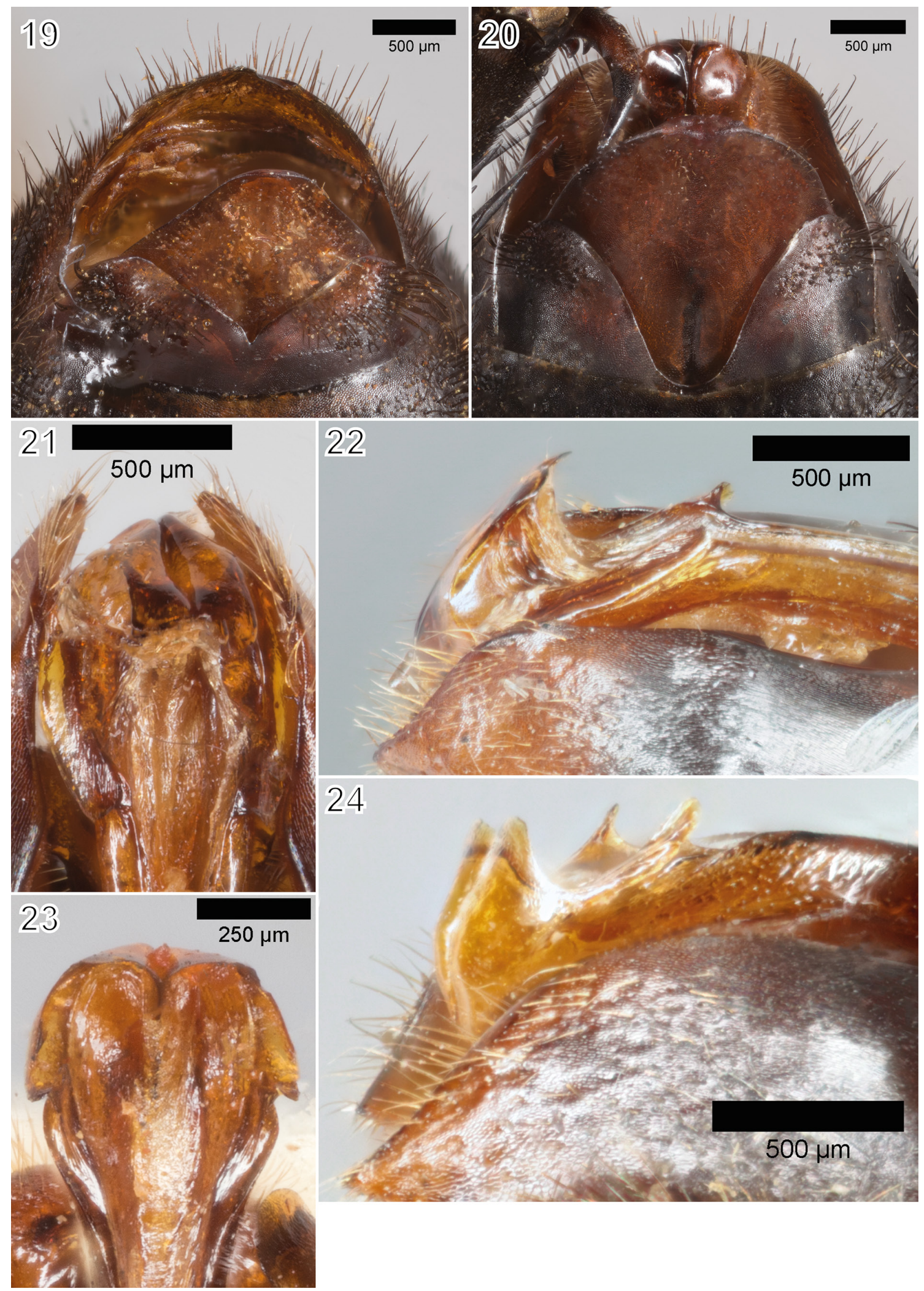

Figs 19-24. 19-20. Ventral view of the two apical sterna in males of the bohemanni group. 21, 23. Dorsal view of apical third of penis valvae in males of the bohemanni group. 22, 24. Lateral view of penis valvae. 19. Sphex bohemanni Dahlbom, 1845. 20. S. abbotti abbotti W. Fox, 1891. 2122. S. stadelmanni stadelmanni Kohl, 1895. 23-24. S. schoutedeni $\mathrm{s}$. lat. 


\section{Key E: Species of the Sphex gaullei group}

1. Female: scutellum and clypeus completely ferruginous (Figs 104, 106, 108). Male: at least lower/inner third of clypeus ferruginous; facial setae golden or silvery

- Female: scutellum and at least upper half of clypeus black (Figs 100-102). Male: clypeus nearly or completely black; facial setae never golden

2. Petiole and metasomal segment I bright ferruginous (Fig. 108). Female: meso- and metapleurae ferruginous; basal half of forewing with conspicuous yellow tinge and cellular wing area not infuscate. Male: erect clypeal and paraocular setae silvery ......S. schmideggeri sp. nov.

- Petiole and metasoma black (Figs 104-107). Female: meso- and metapleurae black; cellular area of forewing either hyaline and without yellow tinge or notably infuscate in apical part with at most inconspicuous yellow tinge near wing base. Male: erect clypeal and paraocular setae golden .......3

3. Female: cellular area of forewing hyaline (Fig. 29), usually without violet iridescence; pronotal lobe predominantly black. Male: hindwing and cellular area of forewing hyaline (Fig. 30) .......

S. jansei Cameron, 1910

- Female: apical half of forewing markedly infuscate (Fig. 31), with violet iridescence; pronotal lobe predominantly ferruginous. Male: cellular area of forewing infuscate in apical half, or at least apical margin of hindwing infuscate (Fig. 32)

S. gaullei Berland, 1927

4. Wings hyaline. Erect facial and propodeal setae silvery (Figs 102-103). Petiole length more than $3 \times$ its medial width S. pruinosus Germar, 1817

- Wings fuscous. Erect facial and propodeal setae black (Figs 100-101). Petiole length less than $2.5 \times$ its medial width

S. decipiens Kohl, 1895

\section{Key F: Species of the Sphex malagassus group}

1. Pronotum laterally and mesopleuron usually with ferruginous stripe. Female: appressed facial setae pale silvery. Male: clypeus entirely ferruginous (Fig. 120)

S. malagassus de Saussure, 1890

- Pronotum and mesopleuron black. Female: appressed facial setae rich golden. Male: clypeus black except for lower center (Fig. 119)

S. comorensis sp. nov.

\section{Key G: Species of the Sphex umtalicus group}

1. Male: appressed clypeal setae black

S. hades sp. nov.

- Male: appressed clypeal setae silvery .2

2. Wings with distinct green iridescence; erect propodeal setae entirely black (Fig. 63). 26-32 mm long ...... S. caeruleanus Drury, 1773

- Wings with violet or blue iridescence, or without any. If greenish, then erect propodeal setae at least partially silvery (Figs 64-65). Smaller

3. Female: erect setae at posterior propodeal margin silvery (Fig. 64). Male: free clypeal margin ferruginous or black; erect propodeal setae largely silvery (Fig. 65); broad placoids cover entire length of at least flagellomeres V and VI .............S. mweruensis (Arnold, 1947)

- Female: erect propodeal setae uniformly black (Figs 69, 72-73). Male: free clypeal margin black; erect propodeal setae black, if silvery then placoids narrow 


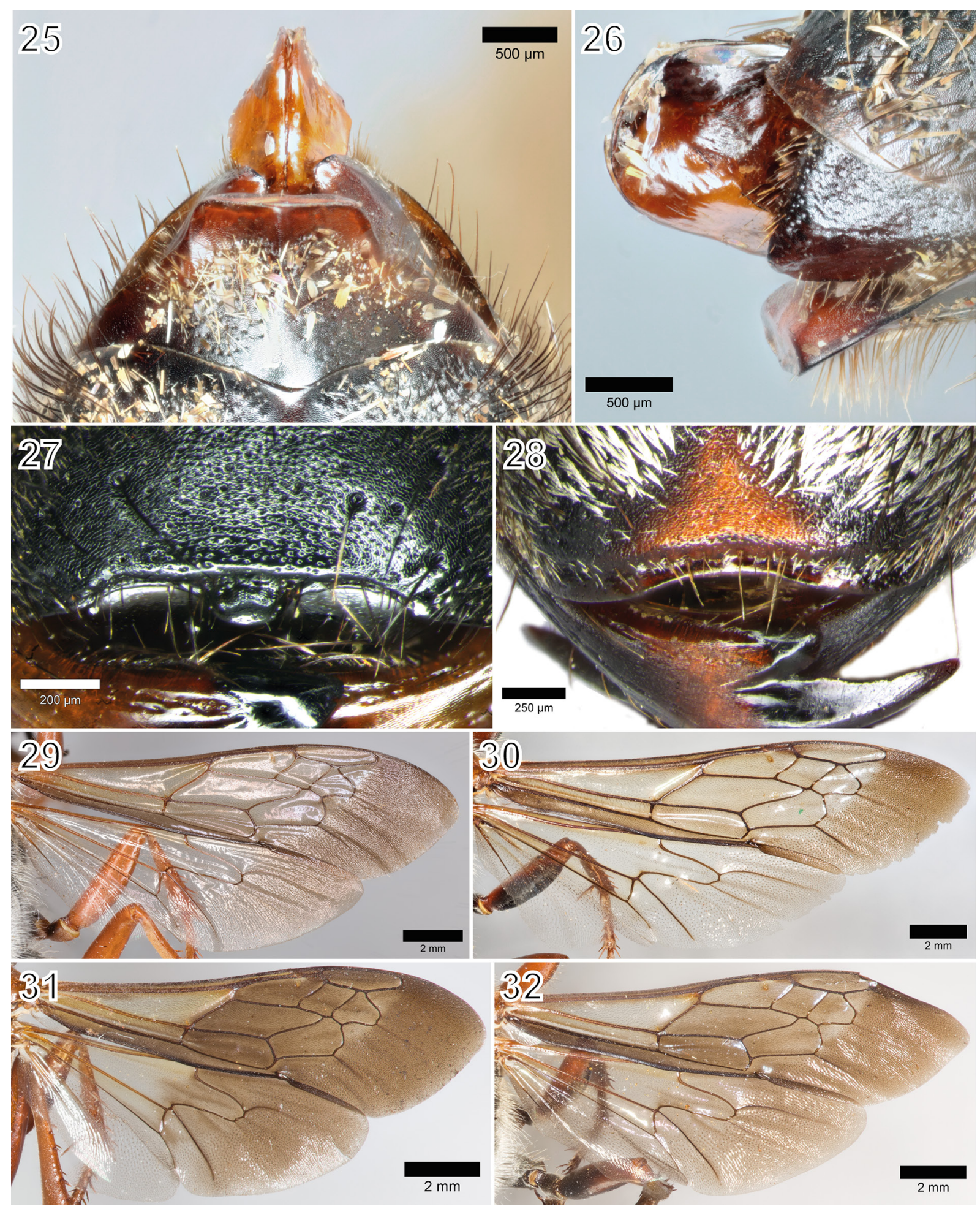

Figs 25-32. 25-26. Ventral and lateral view of sternum VIII and genitalia in male of Sphex abyssinicus (Arnold, 1928). 27-28. Frontal view of free clypeal margin in males. 29-32. Dorsal view of fore- and hindwing in males and females of the gaullei group. 27. S. umtalicus Strand, 1916. 28. S. decipiens Kohl, 1895. 29. S. jansei Cameron, 1910, ‥ 30. S. jansei, đ̃. 31. S. gaullei Berland, 1927, ․ 32. S. gaullei, ô. 
4. Female: clypeus black (Fig. 33), at most lower half ferruginous; legs black, rarely ferruginous (Fig. 72); appressed clypeal setae dark brown; appressed lateroclypeal and paraocular setae without ferruginous-golden luster (Fig. 33); at most basal half of flagellomere I ferruginous. Male: legs black or dark ferruginous, or rarely all tibiae and most of mid- and hindtarsi ferruginous; basal half of mandible black or ferruginous; mesosomal vestiture uniformly black

S. umtalicus Strand, 1916

- Female: clypeus and legs largely ferruginous, if clypeus predominantly black then appressed lateroclypeal and paraocular setae with ferruginous-golden luster (Fig. 34); flagellomeres black or partially ferruginous. Male: basal half of mandible bright ferruginous; hindtibia bright ferruginous with the remainder of the legs black; mesosomal vestiture silvery or black

5. Female: appressed lateroclypeal and paraocular setae with ferruginous-golden luster (Fig. 34). Male: cellular area of fore- and hindwing only slightly fuscous; erect setae at least on scutum partially silvery (Fig. 70); narrow placoid on flagellomere V with at most small placoid structures on neighboring flagellomeres S. haemorrhoidalis Fabricius, 1781

- Female: appressed lateroclypeal and paraocular setae with silvery luster (Fig. 35). Male: wings conspicuously fuscous; erect setae on thoracical dorsum black (Fig. 74); broad placoids cover entire length of flagellomeres IV-VI

S. victoria sp. nov.

\title{
Key H: Species of the Sphex meridionalis group
}

1. Female: scutellum ferruginous (Fig. 127). Male: at least flagellomeres I-IV ferruginous (Fig. 128) S. occidentalis sp. nov.

- Female: scutellum black (Figs 124-125). Male: at most part of flagellomere I ferruginous, remaining flagellomeres black (Fig. 126)

2. Southern-central Africa. Female: appressed paraocular setae rich golden (Fig. 36)

S. meridionalis (Arnold, 1947)

- Eastern Africa. Female: appressed paraocular setae pale silvery-golden (Fig. 37)

S. nefrens sp. nov.

\section{Results}

\author{
Class Insecta Linnaeus, 1758 \\ Order Hymenoptera Linnaeus, 1758 \\ Family Sphecidae Latreille, 1802 \\ Genus Sphex Linnaeus, 1758 \\ Subgenus Sphex (Menkeela) subgen. nov. \\ urn:Isid:zoobank.org:act:757B17B9-BEA9-4588-93F6-CE6348CEBB14
}

Sphex (Menkeella) paulinierii Guérin-Méneville, 1843

Figs 39-40, 47 (blue)

Sphex paulinierii Guérin-Méneville, 1843: 8, † (holotype: †, Senegal, M. Paulinier leg., RMNH, not examined).

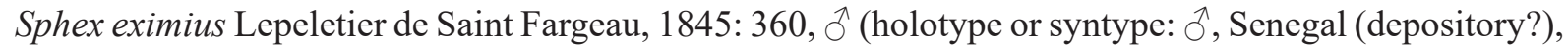
not examined). Synonymized with Chlorion paulinieri by R. Turner 1919: 397. 
Table 3 (continued on next page). Table for quick-identification of males based on placoid configuration. Each flagellomere is represented by three symbols, each of which describes the form of the placoid in the respective approximate third of the segment. If all subspecies have identical placoid configurations, they are combined as sensu lato ('s. lat.'). Notable, but unnamed variations are listed below the respective taxon as 'var.'. The presence of an arrow indicates that different specimens can vary between the states shown, with intermediate forms occurring. The names of new species are highlighted in bold. Legend: '-' = placoid absent; 'o' = placoid narrow; $' \mathrm{O}$ ' = placoid broad

\begin{tabular}{|c|c|c|c|c|c|c|c|c|}
\hline \multirow{2}{*}{ Group } & \multirow{2}{*}{ Species } & \multicolumn{7}{|c|}{ Flagellomere } \\
\hline & & 1 & 2 & 3 & 4 & 5 & 6 & 7 \\
\hline \multirow[t]{10}{*}{ argentatus } & erythrinus & --- & --- & $\mathrm{OOO}$ & $\mathrm{OOO}$ & $\mathrm{OOO}$ & $\mathrm{OOO}$ & --- \\
\hline & feijeni & --- & --- & $\mathrm{OOO}$ & $\mathrm{OOO}$ & OOO & --- & --- \\
\hline & fumicatus & --- & --- & --- & --- & OOO & $\mathrm{OOO}$ & --- \\
\hline & fumicatus var. & --- & --- & --- & $---\rightarrow$ ooo & Ooo & ooo & --- \\
\hline & lanatus & --- & --- & --- & --- & oo- & --- & --- \\
\hline & rufinervis & --- & --- & --- & --- & Ooo & ooo & --- \\
\hline & taschenbergi & --- & ---/OO- & $\mathrm{OOO}$ & $\mathrm{OOO}$ & OOO & $\mathrm{OOO}$ & --- \\
\hline & tomentosus & --- & --- & --- & --- & OOO & $\mathrm{OOO}$ & --- \\
\hline & torridus & --- & --- & --- & --- & $\mathrm{OOO}$ & $\mathrm{OOO}$ & --- \\
\hline & voeltzkowii & --- & --- & --- & --- & $\mathrm{OOO}$ & 000 & --- \\
\hline \multirow[t]{8}{*}{ bohemanni } & abyssinicus & --- & --- & --- & $\mathrm{OOO}$ & $\mathrm{OOO}$ & $\mathrm{OOO}$ & --- \\
\hline & abbotti s. lat. & --- & --- & --- & $\mathrm{OOO}$ & $\mathrm{OOO}$ & $\mathrm{OOO}$ & --- \\
\hline & bohemanni & --- & --- & --- & ooo & Ooo & ooo & --- \\
\hline & bohemanni var. & --- & --- & --- & ooo & Ooo & $---\rightarrow$ o-- & --- \\
\hline & pseudopraedator & --- & --- & --- & $\mathrm{OOO}$ & $\mathrm{OOO}$ & $\mathrm{OOO}$ & --- \\
\hline & pseudopraedator var. & --- & --- & --- & $\mathrm{OOO}$ & $\mathrm{OOO}$ & $\mathrm{OOO}$ & $\mathrm{OOO}$ \\
\hline & schoutedeni $\mathrm{s}$. lat. & --- & --- & --- & $\mathrm{OOO}$ & $\mathrm{OOO}$ & $\mathrm{OOO}$ & --- \\
\hline & stadelmanni $\mathrm{s}$. lat. & --- & --- & --- & $\mathrm{OOO}$ & OOO & $\mathrm{OOO}$ & --- \\
\hline \multirow[t]{7}{*}{ nigrohirtus } & ahasverus & --- & --- & --- & $\mathrm{OOO}$ & $\mathrm{OOO}$ & $\mathrm{OOO}$ & --- \\
\hline & camerunicus & --- & --- & --- & $\mathrm{OOO}$ & OOO & $\mathrm{OOO}$ & $\mathrm{OOO}$ \\
\hline & incomptus & --- & --- & --- & --- & oo- & --- & --- \\
\hline & nigrohirtus & --- & --- & --- & --- & o-o & --- & --- \\
\hline & nigrohirtus var. & --- & --- & --- & --- & ooo/oo- & --- & --- \\
\hline & pulawskii & --- & --- & --- & --- & oo- & --- & --- \\
\hline & pulawskii var. & --- & --- & --- & --- & ooo & $-\mathrm{oo}$ & --- \\
\hline \multirow[t]{10}{*}{ umtalicus } & caeruleanus & --- & --- & --- & OOO/--- & $\mathrm{OOO}$ & $\mathrm{OOO}$ & --- \\
\hline & caeruleanus var. & --- & --- & --- & --- & ooo & ооо & --- \\
\hline & hades & --- & --- & --- & --- & oOo & o-- $\rightarrow$ oOo & --- \\
\hline & haemorrhoidalis & --- & --- & --- & --- & oo- & o-- & --- \\
\hline & haemorrhoidalis var. & --- & --- & --- & --- & oo- & --- & --- \\
\hline & mweruensis & --- & --- & --- & $\mathrm{OOO}$ & OOO & $\mathrm{OOO}$ & --- \\
\hline & umtalicus & --- & --- & --- & --- & oOo & oOo & --- \\
\hline & umtalicus var. & --- & --- & --- & --- & oOo & $---\rightarrow$ o-- & --- \\
\hline & victoria & --- & --- & --- & $\mathrm{OOO}$ & $\mathrm{OOO}$ & $\mathrm{OOO}$ & --- \\
\hline & victoria var. & --- & --- & --- & --- & $\mathrm{OOO}$ & $\mathrm{OOO}$ & --- \\
\hline
\end{tabular}


Table 3 (continued).

\begin{tabular}{|c|c|c|c|c|c|c|c|c|}
\hline \multirow{2}{*}{ Group } & \multirow{2}{*}{ Species } & \multicolumn{7}{|c|}{ Flagellomere } \\
\hline & & 1 & 2 & 3 & 4 & 5 & 6 & 7 \\
\hline \multirow[t]{3}{*}{ meridionalis } & meridionalis & --- & --- & --- & --- & ooo & --- & --- \\
\hline & nefrens & --- & --- & --- & --- & ooo & --- & --- \\
\hline & occidentalis & --- & --- & --- & --- & ooo & --- & --- \\
\hline \multirow[t]{5}{*}{ gaullei } & decipiens & --- & --- & $---\rightarrow$ ooo & ooo/o-o & $---\rightarrow$ oоo & --- & --- \\
\hline & gaullei & --- & --- & ooo/o-o & ooo & --- & --- & --- \\
\hline & jansei & --- & --- & $\mathrm{O}-\mathrm{O}$ & ooo & --- & --- & --- \\
\hline & pruinosus & --- & --- & $\mathrm{OOO}$ & $\mathrm{OOO}$ & $\mathrm{OOO}$ & $\mathrm{OOO}$ & --- \\
\hline & schmideggeri & --- & --- & ooo & ooo & --- & --- & --- \\
\hline \multirow[t]{5}{*}{ satanas } & pseudosatanas & --- & --- & --- & o-- & o-- & o-- & --- \\
\hline & rufoclypeatus & --- & --- & $\mathrm{OOO}$ & OOO & $\mathrm{OOO}$ & OOO & --- \\
\hline & satanas & --- & --- & --- & o-- & o-- & o-- & --- \\
\hline & satanas var. & --- & --- & --- & o-- & o-- & --- & --- \\
\hline & socotrensis & --- & --- & --- & oo- & oo- & oo- & --- \\
\hline \multirow[t]{2}{*}{ malagassus } & comorensis & --- & --- & --- & OOO & OOO & O-- & --- \\
\hline & malagassus & --- & --- & --- & OOO & OOO & OOO & --- \\
\hline \multirow[t]{2}{*}{ incerta sedis } & cinerascens & --- & --- & --- & o-o & ooo & ooo & --- \\
\hline & paulinierii & --0 & OOO & $\mathrm{OOO}$ & OOO & OOO & OOO & --- \\
\hline
\end{tabular}

\section{Differential diagnosis}

Among the African Sphex, this species is very easy to recognize due to its short erect setae on the propodeal enclosure, the two swellings on each propodeal side (Fig. 40), and the conspicuous coloration (Fig. 39) and large body size.

\section{Etymology}

Menkeella is named in honor of Dr Arnold S. Menke.

\section{Material examined}

AFRICA • 1 ô;; "Congo"; MNHN.

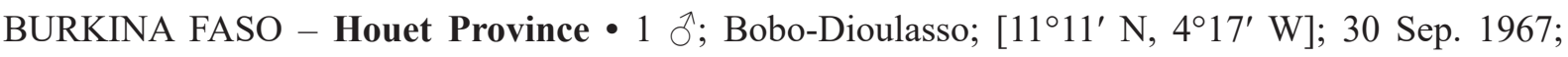
J. Hamon leg.; USNM.

CHAD - Logone Oriental • 1 क ; Bébédjia, 400 m from Moundou; [ $8^{\circ} 41^{\prime}$ N, 16³4' E]; 4 Oct. 1970; J.H.

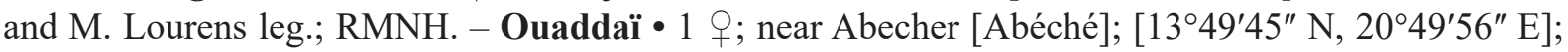
H. Franz leg.; NHMW.

ETHIOPIA 1 ภ̊; BMNH.

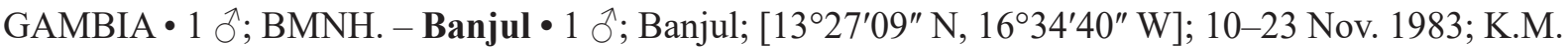
Guichard leg.; BMNH.

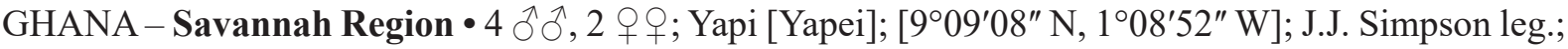
BMNH. 


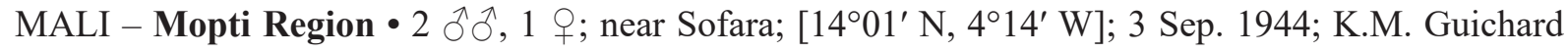
leg.; BMNH.

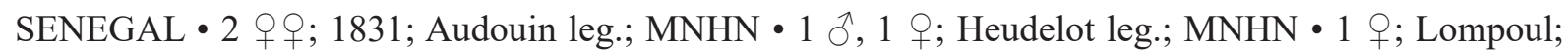

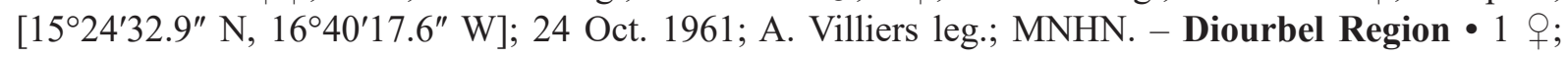

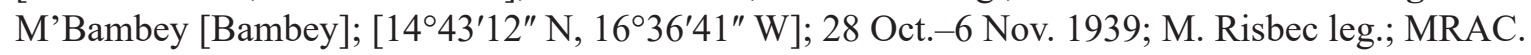

UGANDA - Eastern Region • 1 đ̊’; Mbale-Kumi Road, $3700 \mathrm{ft}$ S of Lake Salisbury; [1 ${ }^{\circ} 33^{\prime} 33^{\prime \prime}$ N, 335'ㄷㄴ" E]; BMNH.

\section{Description}

Female

Size. $34.1-42.5 \mathrm{~mm}$.

CoLOR. Black or dark brown except for the following, which are ferruginous: basal half of mandible, clypeus, scape, pedicel, flagellomeres I-VI, neck, collar, tegula, scutum except anterior margin and medial longitudinal stripe, scutellum, propodeum, petiole dorsally, metasomal segment I, metasomal segment II except posterior margin and legs from coxa onward excluding claw. Cellular wing area yellowish, apex including marginal cell and submarginal cell III of forewing fuscous.

VeSTITURE. Appressed and erect setae on clypeus, paraocular area, collar, scutum and propodeal enclosure rich or pale golden. Erect propodeal setae oriented perpendicularly. Lower center of clypeus with glabrous spot. Scutellum finely and densely pubescent.

STRUCTURE. Free clypeal margin simple, stepped medially. Clypeus without indentation and carina. Scutellum flat. Metanotum not raised, not bituberculate. $2^{\text {nd }}$ recurrent vein joins slightly proximal from interstitium between submarginal cells II and III. Propodeal enclosure with 15-20 distinct ridges. Foretarsomere I $2.5-2.6 \times$ length of antepenultimate spine. Petiole length $2.1-2.2 \times$ its medial width.

\section{Male}

Size. $26.7-32.0 \mathrm{~mm}$.

CoLOR. Black or dark brown, except for the following which are ferruginous: basal half of mandible, clypeus, scape, pedicel, flagellomeres I-VI dorsally, neck and collar dorsally, tegula, scutum, scutellum, propodeal enclosure, petiole, metasomal segment I, metasomal tergum II except posterior margin, apical segment of metasoma and legs from coxa onward excluding claw. Cellular wing area yellowish, apical margin fuscous.

VestituRe. Appressed and erect setae on clypeus, paraocular area, collar and scutum golden, on propodeal enclosure silvery. Erect setae on propodeal margin golden. Erect propodeal setae oriented perpendicularly. Lower center of clypeus with glabrous spot. Scutellum finely and densely pubescent.

STRUCTURE. Free clypeal margin simple. Scutellum convex. Metanotum not raised, not bituberculate. $2^{\text {nd }}$ recurrent vein joins slightly proximal from interstitium between submarginal cells II and III. Propodeal enclosure with 15-20 distinct ridges. Posterior margin of metasomal tergum VII convex. Posterior margin of metasomal sternum VII simple, of metasomal sternum VIII concavely emarginate. Penis valvae without conspicuous modifications. Petiole length $2.4-2.5 \times$ its medial width. Flagellomere I with broad placoid covering distal half, flagellomeres II-VI with broad placoids covering their entire length.

\section{Variation}

Unknown. 

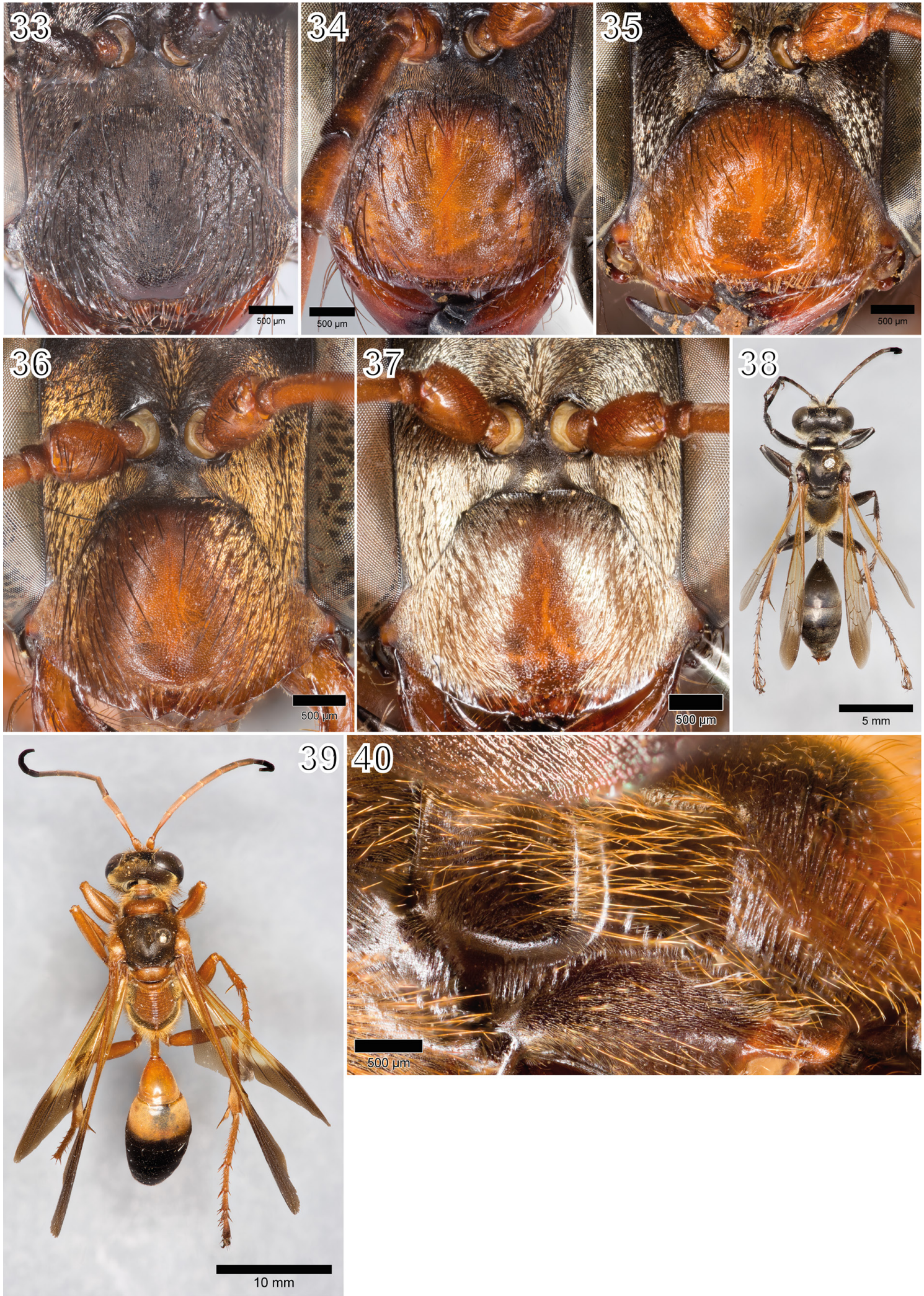

Figs 33-40. 33-37. Frontal view of faces in females of the umtalicus and meridionalis group. 33. Sphex umtalicus Strand, 1916. 34. S. haemorrhoidalis Fabricius, 1781. 35. S. victoria sp. nov. 36. S. meridionalis (Arnold, 1947). 37. S. nefrens sp. nov. 38. S. cinerascens Dahlbom, 1843 habitus of ${ }^{7}$. 39-40. S. paulinierii Guérin-Méneville, 1843, đ̂. 39. Habitus. 40. Mesosomal side (anterior = left). 


\section{Distribution}

Western and eastern Africa.

\section{Remarks}

This species differs from the other Sphex s. str. through its absence of a complete spiracular groove. Instead, it possesses a sulcus that connects the lower metapleural pit with the anteroventral pit (Fig. 40). Similar to members of the argentatus group (Fig. 9), S. paulinierii also has a swelling anteroventrally of the spiracle, although it has an additional swelling posteriorly to it, laterally on the propodeal enclosure. This posterior swelling is unique, and $S$. paulinierii lacks the distinctly impressed, bituberculate metanotum that is characteristic for the argentatus group. Furthermore, its very short erect propodeal setae are unusual for Sphex, and no longitudinal carina is present on the female labrum. For these reasons, with particular emphasis on a complete spiracular groove being an extremely important defining character for the subgenus Sphex (Bohart \& Menke 1976) and the lack of distinctive characteristics for species group assignment, we have decided to place $S$. paulinierii in a separate subgenus.

Subgenus Sphex (Sphex) Linnaeus, 1758

Sphex cinerascens Dahlbom, 1843

Figs 38, 47 (red)

Sphex cinerascens Dahlbom, 1843: 25, ô.

\section{Differential diagnosis}

This species has anteriorly-oriented erect propodeal setae as in members of the gaullei group, though genetic analyses show that it is more distantly related to the gaullei group than members of that group's presumed sister taxon, the satanas group. Thus, $S$. cinerascens is treated separately here.

Females can be distinguished from all other African Sphex by having a conspicuous process medially at the clypeal margin. Males possess a comparable structure, though those of the umtalicus group are also recognizable by having a tooth at the free clypeal margin. However, their erect propodeal setae are oriented perpendicularly, and their erect facial setae are black, whereas they are silvery in S. cinerascens. Both sexes are also characterized through the relative length of their petiole, which measures significantly more than $3.5 \times$ its width. In almost all other species, the ratio between petiole length and width is usually less than 3.2, except for S. pruinosus where this value can be up to 4, but that species is distinguished through its absence of a process at the free clypeal margin and having more or less distinct transversal ridges on the propodeum.

\section{Material examined}

Presumed syntypes (photographs of MZLU specimen examined)

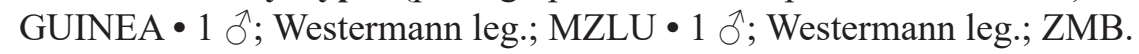

\section{Other material}

AFRICA • 1 ㅇ; W Nile lowlands; Sep. 1929; G.D.H. Carpenter leg.; BMNH.

BENIN - Atlantique Department • $1 \delta^{\Uparrow}$; Godomey; [6 $6^{\circ} 22^{\prime}$ N, $2^{\circ} 21^{\prime}$ E]; 24 Jun. 1968; J. Hamon leg.; MNHN • 1 ; ; same collection data as for preceding but 29 Jun. 1968; MNHN. - Littoral Department •

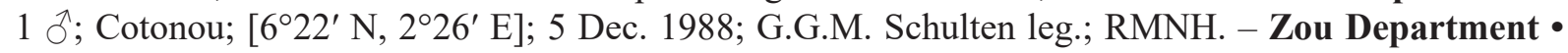
1 '̊; Zangnanado, Ouémé River; [7¹2'59.2" N, 2²8'20.3" E]; 15 Apr. 2000; J. Halada leg.; THD-031OOLM; GenBank CO1 gene: MW538554; OÖLM • 1 ठ̊; same collection data as for preceding; OÖLM.

BURKINA FASO - Cascades Region • 1 q; Tingréla, Banfora; [10³8'31.7" N, 449'50.9” W]; 20 Dec.

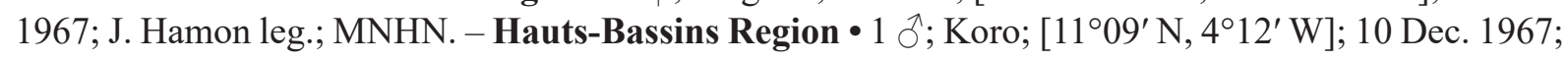


J. Hamon leg.; MNHN • 1 \%; same collection data as for preceding but 18 May 1968; MNHN. - Sahel Region $\bullet 2 \partial^{\prime}$; $15 \mathrm{~km}$ SE of Gorom Gorom; 14²1.4' N, $0^{\circ} 7.9^{\prime}$ W; 28 Jul. 2004; Sidiki Konaté and W.J. Pulawski leg.; CAS • $10^{\top}$; $30 \mathrm{~km}$ SE of Dori; $13^{\circ} 50.3^{\prime} \mathrm{N}, 0^{\circ} 08^{\prime} \mathrm{W}$; 30 Jul. 2004; Sidiki Konaté and W.J. Pulawski leg.; CAS.

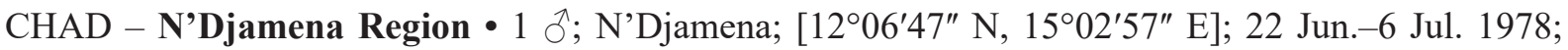
G.G.M. Schulten leg.; RMNH.

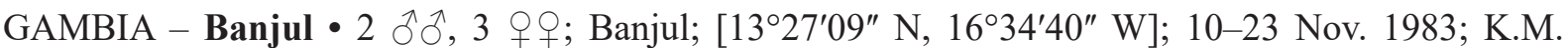

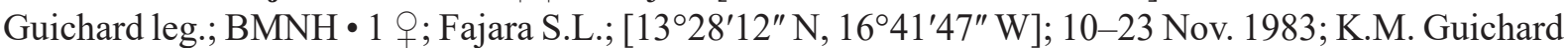
leg.; BMNH.

GHANA - Greater Accra Region • 1 q; Accra; [5³3'21" N, 0¹1'48" W]; 1941; K.M. Guichard leg.;

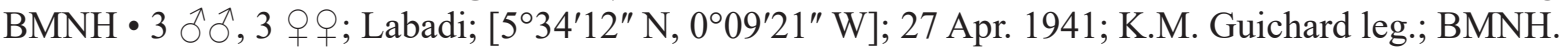

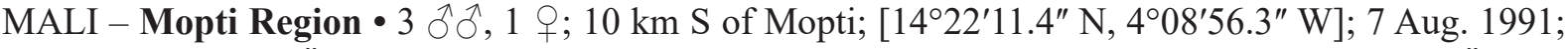

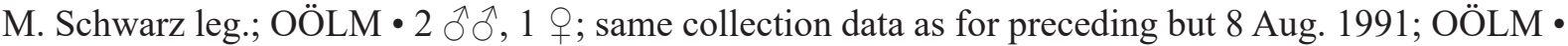
$4 \delta^{\lambda}$; same collection data as for preceding but 10 Aug. 1991; OÖLM • 1 ते; same collection data as for preceding but 20 Aug. 1991; OÖLM. - Ségou Region • 1 क; $60 \mathrm{~km} \mathrm{SW}$ of Ségou; [1300'44" N, 641'18.3" W]; 1 Aug. 1991; M. Schwarz leg.; OÖLM • 1 \%; 70 km SE of Ségou; [1259'29.6" N, 545'45.2" W]; 2 Aug. 1991; M. Schwarz leg.; OÖLM • 1 q; Markala, Niger River; [134ㅇ'26" N, 604'30" W]; 11 Jul. 1968; J. Hamon leg.; MNHN.

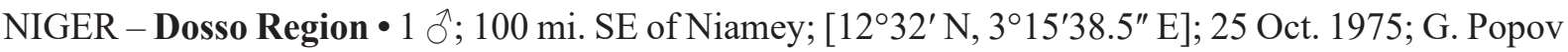
leg.; BMNH.

REPUBLIC OF CÔTE D'IVOIRE - Lagunes District • 2 q $;$; $40 \mathrm{~km} \mathrm{~S}$ of Toumodi; [6 $6^{\circ} 10^{\prime} 37^{\prime \prime} \mathrm{N}$, 456'21" W]; 21 Jan. 1991; W.J. Pulawski leg.; CAS.

SENEGAL - Fatick Region • 1 o; G. de Bandiala; [1339'40" N, 16³4'26" W]; 8-12 Dec. 1997;

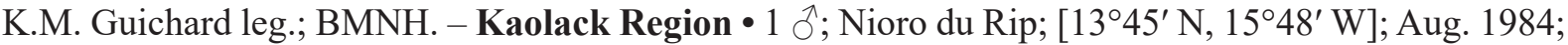
BMNH • 1 ठ̊; Saboya, Nioro; 18 Sep. 1969; J. Hamon leg.; MNHN. - Saint-Louis Region • 1 \%; Richard Toll; [16²8' N, 1541' W]; Nov. 1967; A. Descarpentries, T. Leye and A. Villiers leg.; MNHN.

TOGO - Maritime Region • 1 क; Agbodrafo; [6¹2'05" N, 1²8'44" E]; 10 Nov. 1990; G.G.M. Schulten leg.; RMNH・ 1 q; Lomé, dunes; [607'55" N, 1²13'22" E]; 3 Apr. 1976; G.G.M. Schulten leg.; RMNH.

\section{Description}

Female

Size. $16.4-20.1 \mathrm{~mm}$.

COLOR. Black except for the following, which are ferruginous: basal half of mandible, femora and midand hindlegs from tibia onward. Cellular wing area hyaline, basal wing half with yellow tinge, apical margin of forewing fuscous.

Vestiture. Appressed and erect setae on clypeus, paraocular area, collar, scutum and propodeal enclosure silvery-golden. Erect propodeal setae oriented anteriorly. Clypeus without glabrous spot. Scutellum densely and finely pubescent.

STRUCTURE. Free clypeal margin medially with broad, gently notched tooth, not stepped above. Clypeus without indentation or carina. Scutellum convex. Metanotum slightly raised, not bituberculate. $2^{\text {nd }}$ 
recurrent vein joins markedly proximal from interstitium between submarginal cells II and III. Propodeal enclosure without any notable ridges. Foretarsomere I 2.6-2.8 $\times$ length of antepenultimate spine. Petiole length $3.8-4.1 \times$ its medial width.

\section{Male}

Size. $15.5-18.4 \mathrm{~mm}$.

COLOR. Black except for the following, which are ferruginous: stripe in center of mandible and mid- and hindlegs from tibia onward. Cellular wing area hyaline, basal half with yellow tinge. Apical margin of forewing fuscous.

Vestiture. Appressed and erect setae on clypeus, paraocular area, collar and scutum silvery, on propodeal enclosure silvery-golden. Erect propodeal setae oriented anteriorly. Clypeus without glabrous spot. Scutellum densely and finely pubescent.

Structure. Free clypeal margin with single tooth. Scutellum convex. Metanotum slightly raised, not bituberculate. $2^{\text {nd }}$ recurrent vein joins markedly proximal from interstitium between submarginal cells II and III. Propodeal enclosure without any notable ridges. Posterior margin of metasomal tergum VII convex. Posterior margin of metasomal sternum VII simple, of metasomal sternum VIII triangular. Penis valvae without conspicuous modifications. Petiole length 3.8-5.6× its medial width. Flagellomeres IVVI with narrow placoids, comprising two disconnected parts on IV and being entire on V and VI.

\section{Variation}

Unknown.

\section{Distribution}

Western Africa.

\section{Remarks}

This species is very similar to the Oriental S. obscurus (Fabricus, 1804), with which it had previously been synonymized (van der Vecht 1961). However, locality records and a few characters specific to males suggest that they are actually two different species.

The syntypes of $S$. cinerascens in the collection of the ZMB and MZLU, both males and collected in Guinea by Westermann, along with specimens collected more recently at other Western African localities, differ from Oriental material of S. obscurus in two aspects. Firstly, the African form has a notable tooth medially on the free clypeal margin, which the Oriental species lacks. Secondly, while its placoid pattern seems to be variable, the African species has placoids that cover the entire length of flagellomeres $\mathrm{V}$ and VI, as well as a partial or entire placoid on flagellomere IV or sometimes one on flagellomere III that can be narrowed medially. In contrast, the placoid configuration in two examined Oriental specimens and several individuals from the Arabian Peninsula matches the description in van der Vecht (1961) of the lectotype of S. obscurus, with about the basal 30\% of flagellomeres V and VI being covered.

\section{Species of the argentatus group}

The argentatus group contains the following nine species: Sphex erythrinus, S. feijeni nom. nov., S. fumicatus, S. lanatus, S. rufinervis, S. taschenbergi, S. tomentosus, S. torridus and S. voeltzkowii.

This group was proposed by Hensen (1991), when it primarily encompassed species from the Australis and Orientalis regions. He correctly assigned the Afrotropical S. fumicatus to the group, and 
Schmid-Egger (2014) added S. taschenbergi based on Palearctic records. The species treated here conform to Hensen's diagnostic character, a bituberculate metanotum (Fig. 7). In most species of the group, the tubercles are usually very distinct, but occasional specimens and particularly members of S. erythrinus, S. feijeni nom. nov. and S. rufinervis have them more indistinct. Therefore, we opted to rely on an additional character for species group assignment that was mentioned by Bohart \& Menke (1976): the presence of a linear swelling in front of the upper part of the spiracular groove (Fig. 9). Only members of the argentatus group possess both of these traits, whereas others may have an indistinctly bituberculate metanotum but lack the spiracular swelling (Fig. 10). In the satanas group, the swelling is present, but the metanotum is always unmistakably flat.

Males in the argentatus group seem to have much greater intraspecific size variations than those of other groups. Length differences of up to $\pm 20 \%$ were observed. When sequenced, the differences in the CO1 gene for a small and a large individual of $S$. lanatus were minimal, so any size variations may simply be the result of differing amounts of available food during larval development.

In contrast to all other African species groups of Sphex, the orientation of the erect propodeal setae is not uniform within this group.

Sphex erythrinus (Guiglia, 1939)

Figs 41-42, 45, 47 (yellow)

Chlorion umbrosum var. erythrinum Guiglia, 1939: 201, + , $§$ (authorship attributed to Magretti).

\section{Differential diagnosis}

Sphex erythrinus is similar to the other species of the argentatus group that have silvery mesosomal vestiture: Sphex feijeni nom. nov. (Figs 43-44), S. lanatus (Fig. 50) and S. rufinervis (Fig. 51). However, females of $S$. erythrinus can easily be identified through their yellow-tinged wings (Fig. 41). Regarding males, S. rufinervis is distinguished through its partially black erect facial setae, whereas S. lanatus has the glabrous hindtibial area ferruginous-brown, and only flagellomere $\mathrm{V}$ is partially covered by a placoid. In S. erythrinus and S. feijeni nom. nov., the integument of the legs is black (though, like in most Sphex, the setae on the inner part of the hindtibia are brighter), but the placoids of $S$. erythrinus cover flagellomeres III-VI and are almost as wide as them, whereas the placoids of S. feijeni nom. nov. are only half as wide as the flagellomeres and cover III-V.

\section{Material examined}

Syntypes (total series consists of $15 \hat{\partial} \hat{\partial}, 7$ 우)

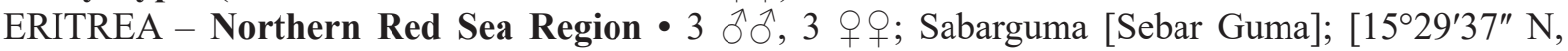
3907'32" E]; Mar. 1900; P. Magretti leg.; MSNG.

\section{Other material}

KENYA - Taita-Taveta County • $3 \partial^{\lambda} \partial^{\lambda}$; Voi; $3^{\circ} 24^{\prime}$ S, 3833.2' E; 21-22 May 2000; V.F. Lee and W.J. Pulawski leg.; THD-024-CAS; GenBank CO1 gene: MW538558; GenBank EF-1 $\alpha$ gene: MW558242;

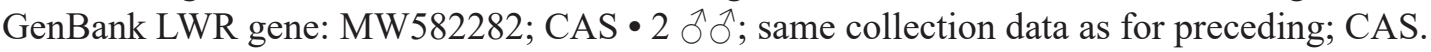

\section{Description}

\section{Female}

Size. 28.3-31.1 mm.

COLOR. Black except for basal half of mandible, which is dark ferruginous. Cellular wing area hyaline with yellow tinge, base of fore- and hindwing infuscate. Forewing with fuscous spot beyond marginal cell. 
VESTITURE. Appressed and erect setae on clypeus, paraocular area, collar, scutum and propodeal enclosure silvery. Erect propodeal setae oriented anteriorly. Clypeus medially with vertical glabrous stripe.

STRUCTURE. Free clypeal margin medially with broad indistinct process, not stepped. Clypeus without indentation or carina. Scutellum convex. Metanotum raised, notably bituberculate. $2^{\text {nd }}$ recurrent vein

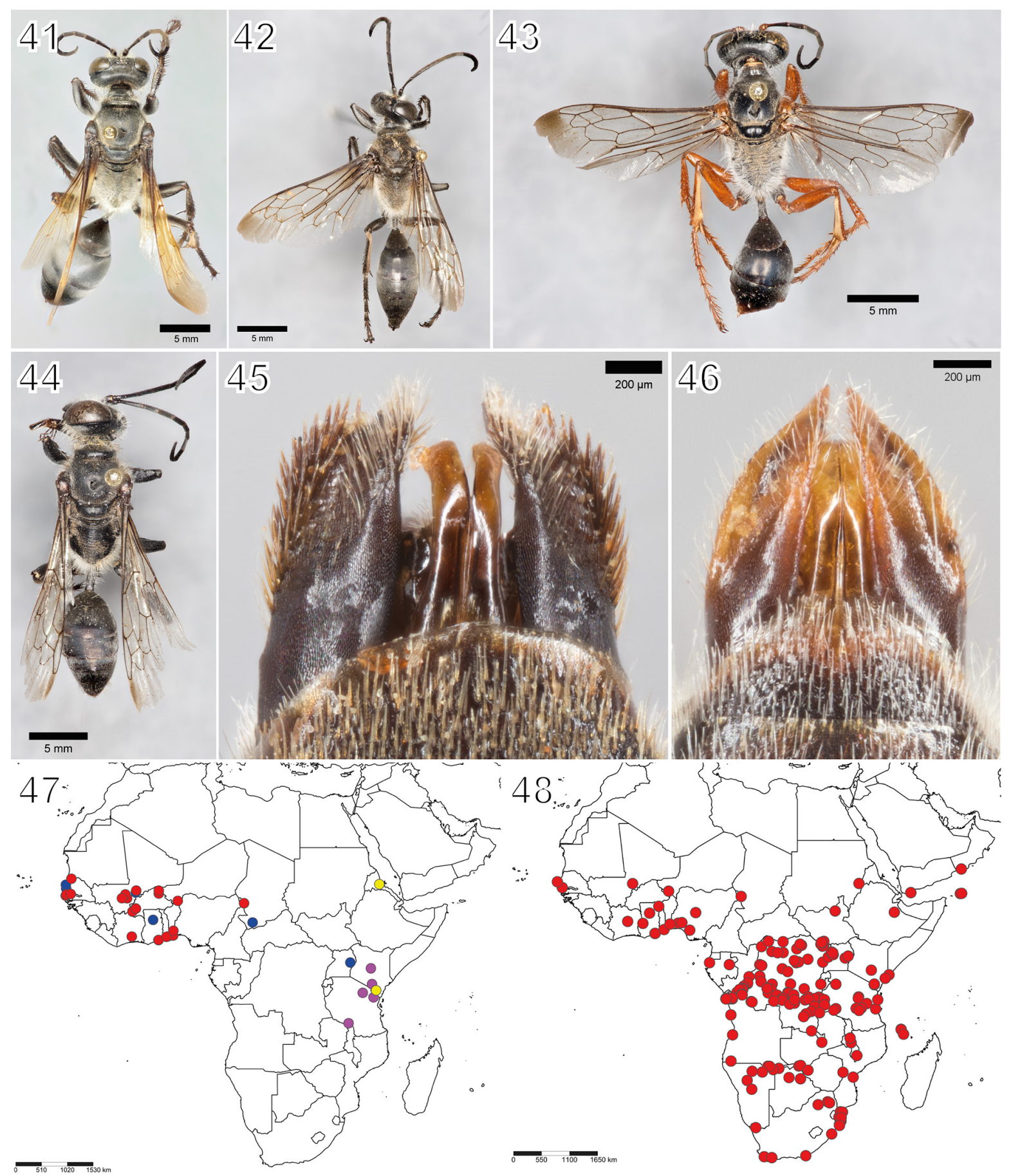

Figs 41-48. 41-42, 45. Sphex erythrinus (Guiglia, 1939). 43-44, 46. Sphex feijeni nom. nov. 41, 43. Habitus of ${ }^{\circ}$. 42, 44. Habitus of 3 . 45-46. Male genitalia. 47. Geographic distribution of S. cinerascens Dahlbom, 1843 (red), S. paulinierii Guérin-Méneville, 1843 (blue), S. erythrinus (yellow) and S. feijeni nom. nov. (purple). 48. Geographic distribution of S. fumicatus Christ, 1791. 
joins slightly proximal from interstitium between submarginal cells II and III. Propodeal enclosure without any notable ridges. Foretarsomere I $2.7-2.9 \times$ length of antepenultimate spine. Petiole length $1.4-1.6 \times$ its medial width.

\section{Male}

SizE. $19.9-23.7 \mathrm{~mm}$.

COLOR. Black. Wings hyaline, apical margin of forewing slightly infuscate.

VeSTITURE. Appressed and erect setae on clypeus, paraocular area, collar, scutum and propodeal enclosure silvery. Erect propodeal setae crimped, oriented anteriorly. Free clypeal margin medially with longitudinal glabrous stripe. Scutellum densely and finely pubescent.

STRUCTURE. Free clypeal margin simple, medially gently notched. Scutellum convex. Metanotum raised, slightly bituberculate. $2^{\text {nd }}$ recurrent vein joins markedly proximal from interstitium between submarginal cells II and III. Propodeal enclosure without any notable ridges. Posterior margin of metasomal tergum VII convex. Posterior margin of metasomal sternum VII simple, of metasomal sternum VIII triangular. Penis valvae without conspicuous modifications. Petiole length $2.2 \times$ its medial width. Flagellomeres III-VI with broad placoids covering their entire length.

\section{Variation}

Unknown.

\section{Distribution}

Eastern Africa.

$$
\begin{aligned}
& \text { Sphex feijeni nom. nov. } \\
& \text { urn:1sid:zoobank.org:act:41616206-05D1-429A-A33B-01189F6B71E7 }
\end{aligned}
$$

Figs 43-44, 46, 47 (purple)

Sphex pruinosus var. haemorrhoidalis Magretti, 1898: 44, +, junior primary honomym of Sphex haemorrhoidalis Fabricius, 1781.

\section{Differential diagnosis}

Together with S. lanatus (Fig. 50) and S. erythrinus (Figs 41-42), this is one of three species in the argentatus group with uniformly silvery-white clypeal and propodeal setae in both sexes. Interestingly, the setae on the propodeum are very attrite in all studied female specimens, leaving large portions of the propodeal enclosure bare. In contrast, S. lanatus and S. erythrinus have much of the propodeal sculpture obscured by dense appressed setae. Furthermore, there are several distinct transversal ridges on the propodeal dorsum of S. feijeni nom. nov. (Fig. 43), a characteristic that is unique among the African members of the argentatus group. What also separates it from the remainder of those species is the fact that in females, approximately the lower two-thirds of the clypeus are brightly ferruginous, whereas the others have it completely black.

The distinguishing characteristics of males (Fig. 44) are more subtle. From S. lanatus, they differ in having a notably shorter petiole, the length of which (see Introduction to measurements) reaches no more than 2.5 times its medial width instead of almost 3 times. Sphex feijeni nom. nov. also has a distinct placoid pattern, with placoids that are half as wide as the segments covering flagellomeres III-V. The very similar S. erythrinus has the placoids nearly as wide as the segments and covering flagellomere VI in addition to III-V, while S. lanatus has only a partial, narrow placoid on flagellomere V. Finally, 
if genitalized, S. feijeni nom. nov. is easily distinguishable through its uniformly hyaline setae laterally on the gonostyles (Fig. 46), as both of the other species have the stout lateral setae black or dark brown (Fig. 45), with only the thin setae near the apex being silvery.

\section{Etymology}

The species name is dedicated to Dr Hans R. Feijen, who collected the majority of the specimens examined here.

\section{Material examined}

Holotype (photographs examined)

SOMALIA • + ; MSNG.

Other material

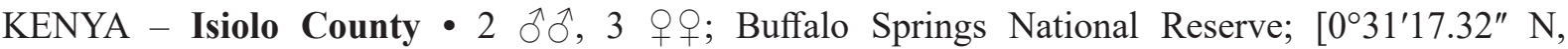
37³7'2.53" E]; 15-18 Jul. 1987; H.R. Feijen leg.; RMNH • 1 ó; same collection data as for preceding;

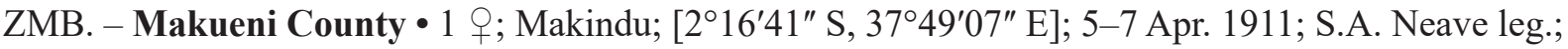
BMNH.

TANZANIA - Manyara Region • $10^{\top}$; Angleheardt Bridge, Tarangire National Park; $3^{\circ} 50^{\prime} \mathrm{S}, 36^{\circ} 10^{\prime} \mathrm{E}$; 16 Mar. 1995; Lesio and Liseki leg.; AMNH. - Mbeya Region • 1 \%; Langenburg [now Tukuyu]; [9 $9^{\circ} 5^{\prime} \mathrm{S}$, 3339’ E]; 25 May 1899; F. Fülleborn leg.; ZMB. - Tanga Region • 1 ơ; 2 km NE of Mkomazi; $4^{\circ} 37.8^{\prime}$ S, 3805.5' E; 29-31 Dec. 2002; W.J. Pulawski leg.; CAS.

\section{Description}

\section{Female}

Size. 20.8-26.3 mm.

COLOR. Black except for the following, which are ferruginous: basal half of mandible, lower part of clypeus, scape, pedicel, basal half of flagellomere I anteriorly, legs from trochanter onward, terga V-VI and sterna V-VI. Cellular wing area hyaline, forewing with fuscous spot beyond marginal cell.

VeSTITURE. Appressed and erect setae on clypeus, paraocular area, collar, scutum and propodeal enclosure silvery. Erect propodeal setae oriented anteriorly. Central third of clypeus glabrous. Scutellum densely and finely pubescent.

StruCture. Free clypeal margin stepped. Clypeus without indentation or carina. Scutellum convex. Metanotum raised, slightly bituberculate. $2^{\text {nd }}$ recurrent vein joins markedly proximal from interstitium between submarginal cells II and III. Propodeal enclosure with approximately seven transversal ridges. Foretarsomere I 1.8-2.1 $\times$ length of antepenultimate spine. Petiole length $1.8-2.0 \times$ its medial width.

\section{Male}

SizE. 16.9-20.6 mm.

COLOR. Black except for ferruginous-brown stripe in center of mandible. Cellular wing area hyaline, forewing with fuscous spot beyond marginal cell.

Vestiture. Appressed and erect setae on clypeus, paraocular area, collar, scutum and propodeal enclosure silvery. Erect propodeal setae oriented anteriorly. Clypeus medially with vertical glabrous stripe. Scutellum densely and finely pubescent.

STRUCTURE. Free clypeal margin slightly stepped medially. Scutellum convex. Metanotum raised, slightly bituberculate. $2^{\text {nd }}$ recurrent vein joins slightly to markedly proximal from interstitium between 
submarginal cells II and III. Propodeal enclosure without any notable ridges. Posterior margin of metasomal tergum VII convex. Posterior margin of metasomal sternum VII simple, of metasomal sternum VIII very slightly concavely emarginate. Penis valvae without conspicuous modifications. Petiole length $2.0-2.3 \times$ its medial width. Flagellomeres III-V with moderately broad placoids covering their entire length.

\section{Variation}

In the holotype, scutum and scutellum are ferruginous instead of black.

\section{Distribution}

Eastern Africa.

\section{Remarks}

Giordani Soika (1942) synonymised this species with S. rufiscutis (R. Turner, 1918), which is a synonym of S. jansei Cameron, 1910. The holotype of S. pruinosus var. haemorrhoidalis Magretti, 1898 is in poor condition, but several characters clearly contradict Giordani Soika's assessment. Firstly, the type has the upper part of the clypeus black, the lower part ferruginous and the collar black, whereas female $S$. jansei have the entire clypeus as well as the collar ferruginous. Also, the free clypeal margin is largely straight in Magretti's species, while it is notably convex in $S$. jansei. The transversal ridges on the propodeum of $S$. jansei are very faint, but those of S. pruinosus var. haemorrhoidalis are sharply distinct. Contrary to Magretti's assumption, the species is not closely allied with S. pruinosus, as S. pruinosus var. haemorrhoidalis has a distinctly shorter and wider petiole and a large glabrous spot on the lower part of the clypeus. While the members of the gaullei group have a somewhat raised and bituberculate metanotum as well, the presence of a swelling anterior to the spiracular groove shows that $S$. pruinosus var. haemorrhoidalis is a member of the argentatus group. Since Magretti's name is an invalid junior homonym of S. haemorrhoidalis Fabricius, 1781, we propose feijeni nom. nov. as a replacement name.

Sphex fumicatus Christ, 1791

Figs 48-49, 53

Sphex fumicatus Christ, 1791: 295, sex not stated (holotype or syntypes: origin unknown, destroyed). Sphex albifrons Fabricius, 1793: 207, sex not stated, junior primary homonym of Sphex albifrons de Villers, 1789 (holotype:, , Guinea, ZMUC, not examined). Synonymized with Sphex metallicus by van der Vecht 1961: 29.

Sphex metallicus Taschenberg, 1869: 414,, , $\widehat{\jmath}$ (syntypes: Sudan, Khartoum State, Khartoum, HALLE, not examined). Synonymized with Sphex fumicatus by van der Vecht 1973: 344.

Sphex magrettii Gribodo, 1894: 136, o (syntypes: 1 ㅇ, Mozambique, Inhambane Province, Inhambane River, Mus. Zool. Univ. Bologna; 1 , Tanzania, Mjini Magharibi Region, Zanzibar, lost. Not examined).

Sphex erebus W.F. Kirby, 1900: 15, sex not stated (syntypes: Yemen, Socotra Governorate, Hadibu [Hadiboh] Plain, BMNH, not examined). Synonymized with Sphex umbrosus by Kohl 1906: 199.

Chlorion davisi Fernald, 1907: 267, ㅇ (holotype: ㅇ, Argentina, Córdoba Province, Córdoba, in Museum of Comparative Zoology (Harvard University, Cambridge, MA, USA), but locality label in error (Menke 1962: 63), not examined). Synonymized with Sphex metallicus by Menke 1962: 63.

\section{Differential diagnosis}

This species can easily be distinguished from other members of the argentatus group, characterized by their bituberculate metanotum, through the combination of notably infuscate forewing bases, black erect propodeal setae and uniformly silvery erect clypeal and paraocular setae (Figs 49, 53), though some of 
the erect facial setae can occasionally have darkened tips. Sphex taschenbergi shares the fuscous wing base and is very similar (Fig. 52), but most or all of its erect clypeal and paraocular setae are entirely black in both sexes (Fig. 54).

\section{Material examined}

AFRICA • 1 §̊; N of Boucle du Niger; Aug. 1910; J. Vuillet leg.; MNHN.

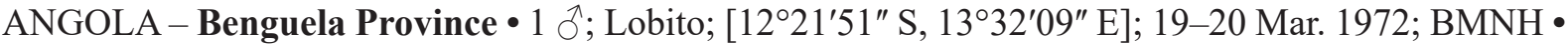
$1 \mathrm{O}^{\top}$; Lobito Bay; [12²1'51" S, 1332'09" E]; 19-20 Jul. 1931; W.P. Cockerell leg.; BMNH. - Luanda Province • 1 \%; Luanda; [ $8^{\circ} 50^{\prime} 18^{\prime \prime}$ S, $13^{\circ} 14^{\prime} 04^{\prime \prime}$ E]; 30 Nov. 1966; E.S. Ross and K. Lorenzen leg.; CAS.

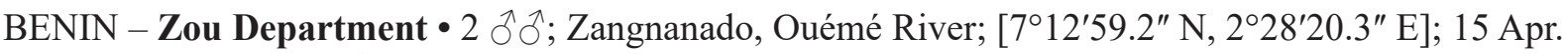
2000; J. Halada leg.; OÖLM.

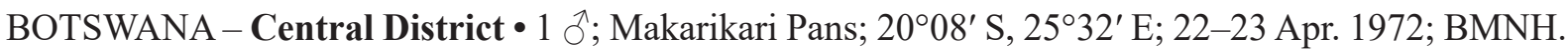
- North-West District • 1 \%; Island Safari Lodge, Maun; [1955'27.7" S, 2330'31.2" E]; Jan. 1997;

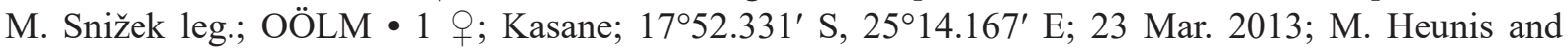
A. Heunis leg.; ZMB.

CAMEROON • 1 \%; M. Boulard leg.; MNHN.

CENTRAL AFRICAN REPUBLIC • 1 क; M. Boulard leg.; MNHN.

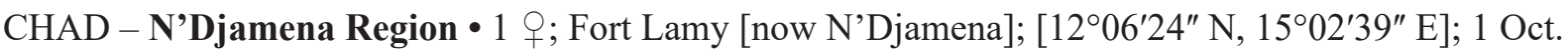
1955; BMNH.

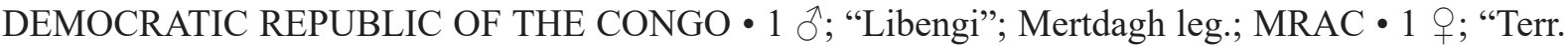
Kasongo, Riv. Lumami”; Sep. 1959; P.L.G. Benoit leg.; MRAC • 1 + ; "Ubangi: Nzali”; 3-4 Feb. 1932; H.J. Brédo leg.; MRAC • 2 q ; ; “Ubangi: Tongu”; 4 Feb. 1932; H.J. Brédo leg.; MRAC・ 1 q; Gilson leg.; MRAC • 1 क; Katanga, Kikva; Apr. 1931; H.J. Brédo leg.; MRAC • 1 \%; Katanga, Luilu; Dec.

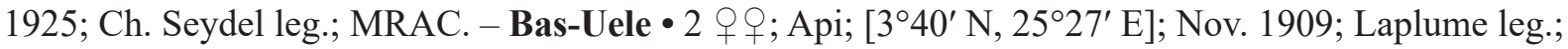

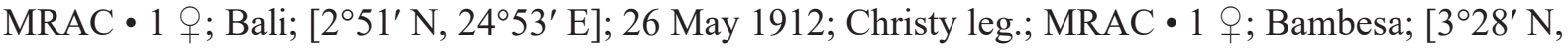
254' E]; Nov. 1930; J. Vrijdagh leg.; MRAC • 1 ; ; same collection data as for preceding but Oct. 1937; MRAC • 1 đ; ; same locality as for preceding; 30 Oct. 1933; H.J. Brédo leg.; MRAC • 2 O $O$; same collection data as for preceding but Dec. 1933; MRAC $\bullet 1$; same locality as for preceding; 9 May

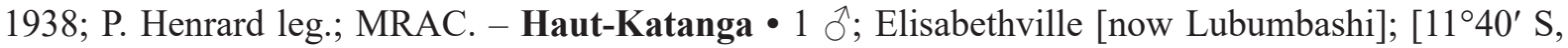

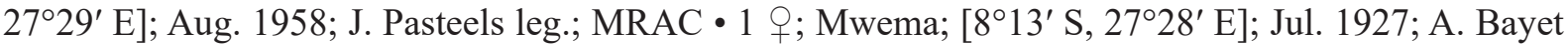
leg.; MRAC • 1 क; Pweto; [8²8' S, 2854' E]; Oct. 1926; A. Bayet leg.; MRAC. - Haut-Lomami • 1 q; Kanonga (675 m), P.N.U. [Upemba National Park]; [909' S, 2605' E]; 14-23 Feb. 1949; G.F. de Witte leg.; MRAC • 1 q; Mabwe (Lac Ubemba, R.E.), P.N.U. [Upemba National Park]; [8 $8^{\circ} 39^{\prime} 10^{\prime \prime}$ S, 26³0'32" E]; 6 Mar. 1949; G.F. de Witte leg.; MRAC • 1 क; same collection data as for preceding but Mar. 1949; MRAC. - Haut-Uele • 1 q; "P.N.G., Miss. H. De Saeger" [Garamba National Park];

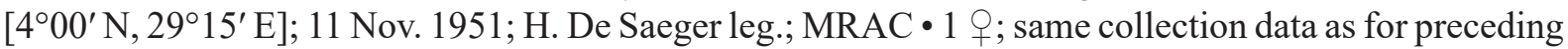
but 1 Mar. 1952; MRAC • 1 क ; Faradje; $3^{\circ} 40^{\prime}$ N, 2940' E; Apr. 1911; Lang and Chapin leg.; AMNH • 2 우; same collection data as for preceding but Dec. 1912; AMNH • 4 우; same collection data as for preceding but Jan. 1913; AMNH - 1 q; Gangala-na-Bodio, Station élevage Elephants; [3 $3^{\circ} 40^{\prime} 47^{\prime \prime} \mathrm{N}$,

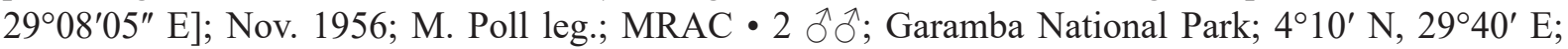
Jun.-Jul. 1912; Lang and Chapin leg.; AMNH 1 q; Vankerkhovenville; [ $3^{\circ} 18^{\prime} \mathrm{N}, 29^{\circ} 26^{\prime}$ E]; Degreef leg.; MRAC. - Ituri Province • 1 क ; Lac Albert, Kasenyi; [1 ${ }^{\circ} 23^{\prime} 31^{\prime \prime}$ N, 30²6'24" E]; 15 May 1935; H.J. Brédo leg.; MRAC • 1 \%; same collection data as for preceding but Jun. 1935; MRAC • 1 \%; Mahagi;

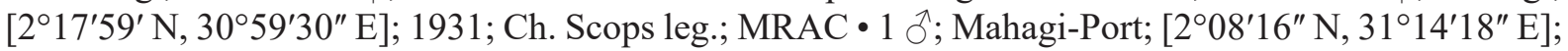


Oct. 1934; H.J. Brédo leg.; MRAC • 1 đ̧; same collection data as for preceding but Oct. 1935; MRAC.

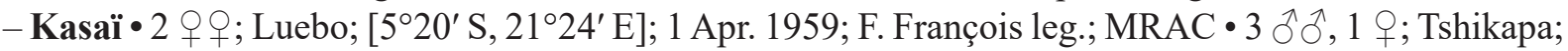

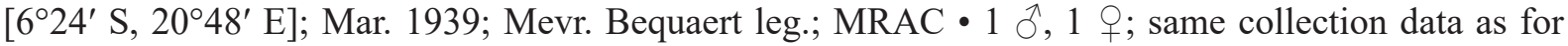
preceding but Apr. 1939; MRAC • 3 우; same collection data as for preceding but Apr.-May 1939;

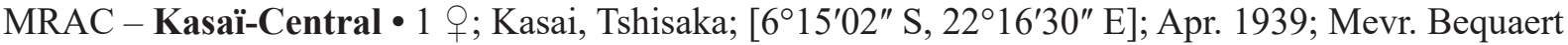

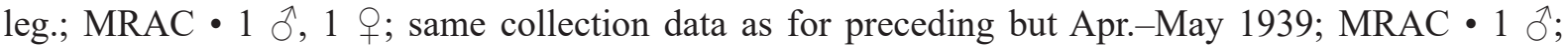

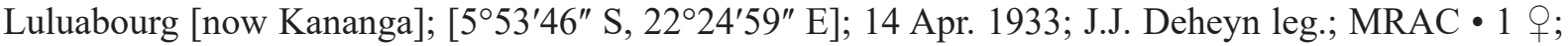

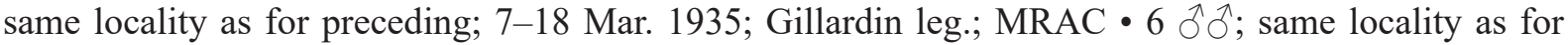
preceding; 17 May 1919; P. Callewaert leg.; MRAC 1 ㄱ, 2 우; same collection data as for preceding; MRAC. - Kasaï-Oriental • $1{ }^{\top}$; Gandajika; [6 $6^{\circ} 45^{\prime}$ S, 235' E]; 31 Jan. 1951; P. de Francquen leg.; MRAC • 1 क; same collection data as for preceding but 1952; MRAC • 1 q; same collection data as for preceding but Dec. 1953; MRAC • 4 ठ̂̉; same collection data as for preceding but 1956; MRAC •

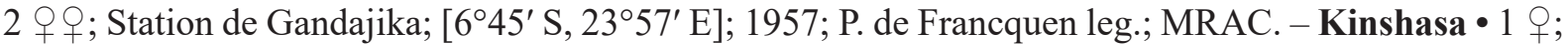

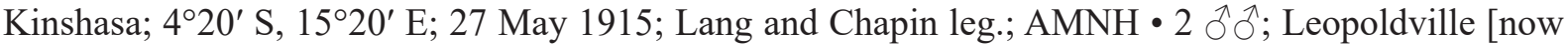

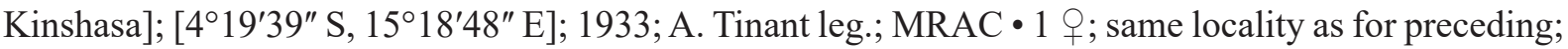

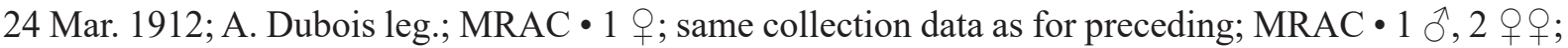

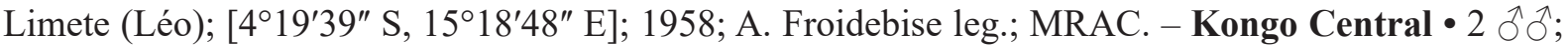
Banana; $6^{\circ} \mathrm{S}, 12^{\circ} 20^{\prime} \mathrm{E}$; Aug. 1915; Lang and Chapin leg.; AMNH 11 , 2 우; same collection data as for preceding but Sep. 1915; AMNH • 1 q; Banana; [601' S, 12 ${ }^{\circ} 24^{\prime}$ E]; Apr. 1918; R. Verschueren leg.;

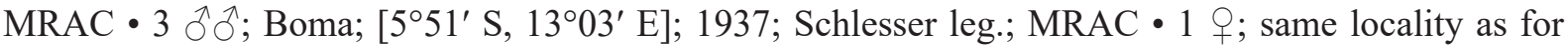
preceding; 24 Mar. 1913; Styczynski leg.; MRAC • 1 đo; same locality as for preceding; 19 Jun. 1915; J. Bequaert leg.; MRAC • 1 O; same locality as for preceding; 16 Jan. 1946; J. Vrydagh leg.; MRAC •

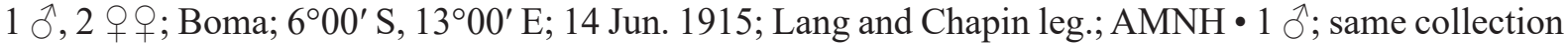
data as for preceding but $16 \mathrm{Jun} .1915 ; \mathrm{AMNH} \bullet 4 \widehat{\widehat{\partial}}$; same collection data as for preceding but $17 \mathrm{Jun}$.

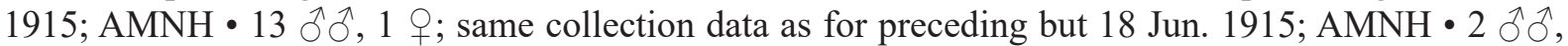
2 우; same collection data as for preceding but 19 Jun. 1915; AMNH 2 o $9+$; Kanzi (Boma); [552' S,

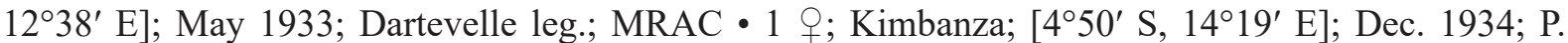

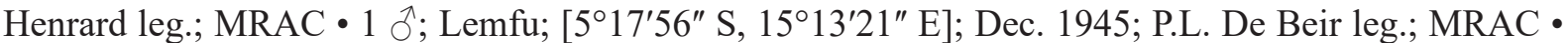
$1 \mathrm{O}^{\top}$; Matadi; $6^{\circ} 00^{\prime} \mathrm{S}, 13^{\circ} 30^{\prime} \mathrm{E}$; 9 Jun. 1915; Lang and Chapin leg.; AMNH • 1 O; Matadi; [549' S,

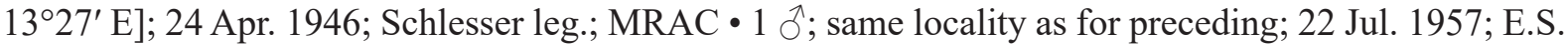

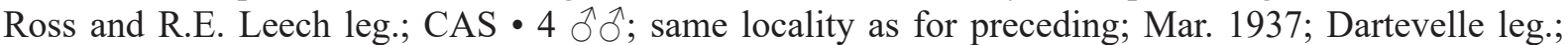

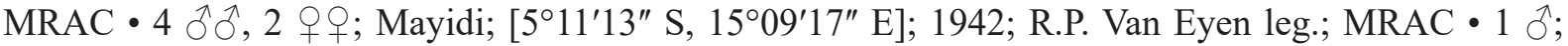
Moanda; [556' S, 12²1' E]; 24 Aug. 1920; H. Schouteden leg.; MRAC. - Kwango • 1 q; Kasongo-

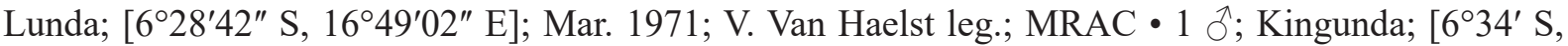
165' E]; 17 Jan. 1939; Mevr. Bequaert leg.; MRAC. - Kwilu Province • 1 q; "Kwango: Atene";

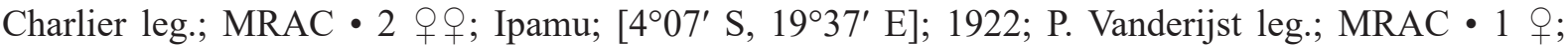
Mwilambongo (Idiofa); [456'36" S, 1948'18" E]; 1947; Soeur Imelda leg.; MRAC. - Lomami • 1 ; ;

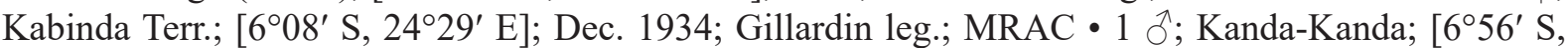

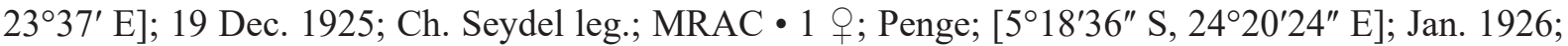
Ch. Seydel leg.; MRAC 1 क; Tshaba; [6 $6^{\circ} 46^{\prime}$ S, 24²2' E]; Dec. 1925; Ch. Seydel leg.; MRAC. - Mai-

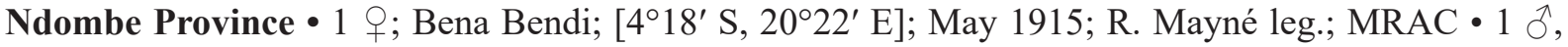

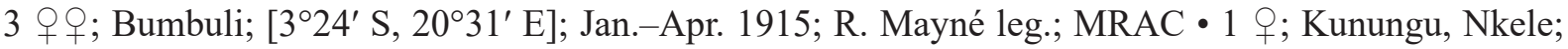

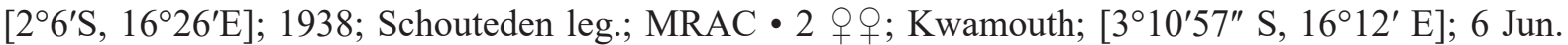
1913; J. Maes leg.; MRAC • 2 우우 Kwamouth; $3^{\circ} 20^{\prime}$ S, $16^{\circ} 10^{\prime}$ E; 24 May 1915; Lang and Chapin leg.; AMNH • 1 क; Lake Leopold II [now Lake Mai-Ndombe]; [2 $2^{\circ} 00^{\prime} \mathrm{S}, 18^{\circ} 20^{\prime}$ E]; Van Hollebeke leg.; MRAC • 2 우; Tolo; [257'14" S, 1834'08" E]; Dec. 1913; J. Maes leg.; MRAC. - Maniema • 3 우;

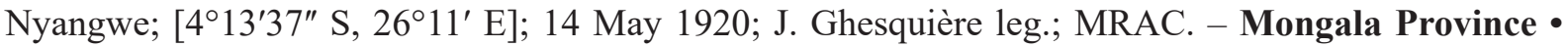

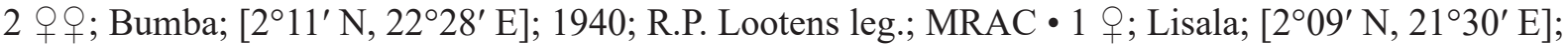
1949; Leontowitch leg.; MRAC. - Nord-Ubangi • 2 우; Bosobolo; [4¹1'23" N, 1952'59" E]; 8-11 Jan. 1932; H.J. Brédo leg.; MRAC • 3 우; Yakoma; [405'53" N, 22²6'45" E]; 5-17 Feb. 1932; H.J. 
Brédo leg.; MRAC. - North Kivu • 1 q; Beni; [ $0^{\circ} 29^{\prime}$ N, 29²8' E]; Borgerhoff leg.; MRAC. - Province

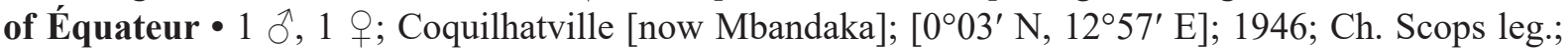

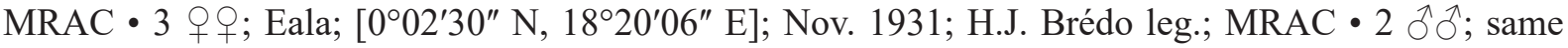
collection data as for preceding but Mar. 1932; MRAC • 1 \%; same locality as for preceding; Jun. 1932; A. Corbisier leg.; MRAC. - Sankuru • 1 q; Inkongo, Lusambo; [453' S, 2318' E]; 1929; Wilson leg.;

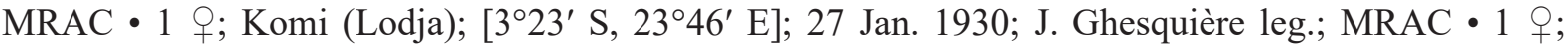
Kondue; [ $\left.4^{\circ} 58^{\prime} \mathrm{S}, 23^{\circ} 16^{\prime} \mathrm{E}\right]$; 1934; Puissant leg.; MRAC 11 ; ; same collection data as for preceding but Leonhard leg.; MRAC • 1 क; Lusambo; [458' S, 2327' E]; Jan. 1926; Ch. Seydel leg.; MRAC •

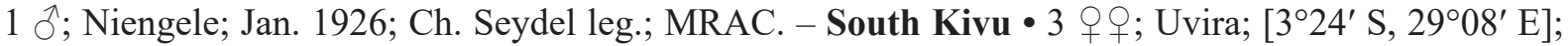
Sep. 1958; J. Pasteels leg.; MRAC • 1 ðं; same locality as for preceding; 24-28 Dec. 1952; P. Basilewsky leg.; MRAC • 1 đ̊; same collection data as for preceding but 16-23 Mar. 1953; MRAC • 1 क; same locality as for preceding; Oct. 1927; Ch. Seydel leg.; MRAC. - Sud-Ubangi • 5 우; Binga; $\left[2^{\circ} 22^{\prime}\right.$ N,

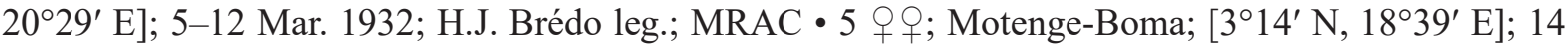
Dec. 1931; H.J. Brédo leg.; MRAC. - Tanganyika - 1 o; Albertville [now Kalemie]; [556'50" S, 29¹1'40" E]; Dec. 1918; R. Mayné leg.; MRAC • 1 q; Kabalo; [603' S, 265' E]; 1 Jul. 1947; M. Poll leg.; MRAC • 1 q; Katompe; [6¹1'55" S, 26²1'28" E]; 20 Dec. 1923; M. Bequaert leg.; MRAC • 1 q;

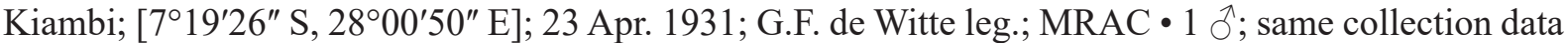
as for preceding but 27 Apr. 1931; MRAC • 1 क ; same collection data as for preceding but May 1931;

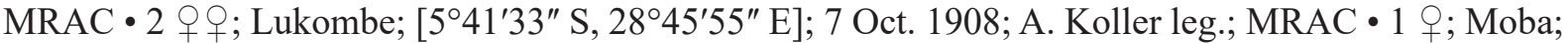
[704' S, 2946' E]; Apr. 1954; H. Bomans leg.; MRAC • 1 क; Mpala; [643'56" S, 29³1'28" E]; Jul.Aug. 1953; H. Bomans leg.; MRAC • 1 q; Nyunzu; [557' S, 2801' E]; Jan.-Feb. 1934; H. De Saeger leg.; MRAC • 1 q; Vallée Lukuga; [5³9'31" S, 2654'53" E]; Nov. 1911; Schwetz leg.; MRAC. Tshopo • 1 q; Kamunionge; $1^{\circ}$ N, 26 $6^{\circ} 50^{\prime}$ E; 24 May 1915; Lang and Chapin leg.; AMNH • 1 ; ; Stanleyville [now Kisangani]; $0^{\circ} 30^{\prime} \mathrm{N}, 25^{\circ} 10^{\prime} \mathrm{E}$; Feb. 1915; Lang and Chapin leg.; AMNH 1 o 1 , 1 ; same collection data as for preceding but Apr. 1915; AMNH • 1 ; ; same collection data as for preceding but 10 Apr. 1915; AMNH • 1 O; same collection data as for preceding but 15 Apr. 1915; AMNH 1 \%; same collection data as for preceding but 17 Apr. 1915; AMNH • 1 ㅇ; Stanleyville [now Kisangani]; [0³0'55" N, 2511'27" E]; Apr. 1915; J. Bequaert leg.; MRAC • 1 q; same locality as for preceding; 1 Aug. 1932; J. Vrydagh leg.; MRAC - 1 q; Yalikanda; [0²3' N, 2447' E]; 20 Feb. 1971; M. IsySchwart leg.; MNHN • 1 q; Yangambi; [0 $46^{\prime}$ N, 24²7' E]; May 1960; J. Decelle leg.; MRAC. Tshuapa • 1 ${ }_{+}$; Bokuma; [006' S, $18^{\circ} 41^{\prime}$ E]; Jan.-Feb. 1952; R.P. Lootens leg.; MRAC • 1 \%; Bokungu; $\left[0^{\circ} 41^{\prime} \mathrm{S}, 22^{\circ} 19^{\prime} \mathrm{E}\right]$; 1950; M. Dupuis leg.; MRAC • 1 q; Ikela; [1 ${ }^{\circ} 11^{\prime} \mathrm{S}, 23^{\circ} 16^{\prime}$ E]; 1955; R.P. Lootens leg.; MRAC $\bullet 2$ q $O$; same collection data as for preceding but 1956; MRAC $\bullet 1$; ; same locality as for preceding; Mar.-Jun. 1956; R. Deguide leg.; MRAC.

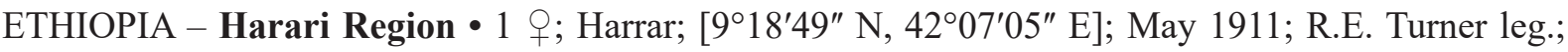
BMNH.

GABON • 1 o; Theorin leg.; NRS. - Estuaire Province • 1 q; Libreville; [0²3'24.36" N, 9²7'15.84" E]; 1936; MNHN.

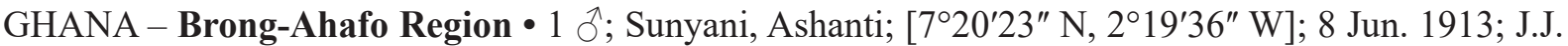

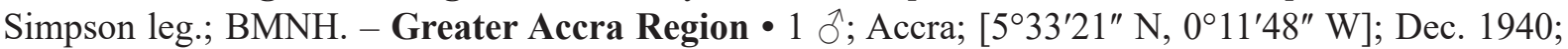
K.M. Guichard leg.; BMNH 1 o ; same collection data as for preceding but Aug.-Sep. 1941; BMNH •

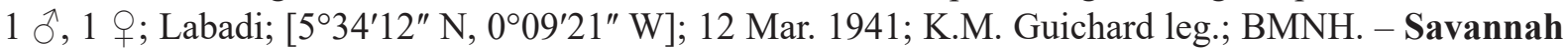

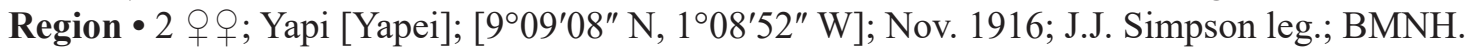

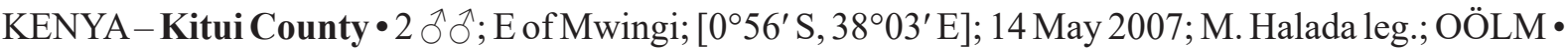

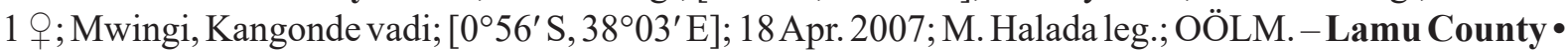
1 क; Boni Forest area, near Mangai Village; $1.74872^{\circ}$ S, 41.17820 E; 10-12 Jun. 2013; J. Bukhebi and 
R. Copeland leg.; ICIPE • 1 क; Uchweni Forest; [2²1'57" S, 40³0'29" E]; 1-2 Mar.1912; S.A. Neave leg.; BMNH. - Taita-Taveta County • 1 q; Sagalla Swamp; [3³0'34" S, 38³4'34" E]; 1947; BMNH • 1 ô;$^{\prime}$ near Voi (Tsavo); [3²3'26" S, 38³4'37" E]; 22 Nov.-2 Dec. 1996; Mi. Halada leg.; OÖLM.

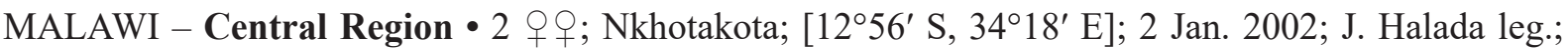

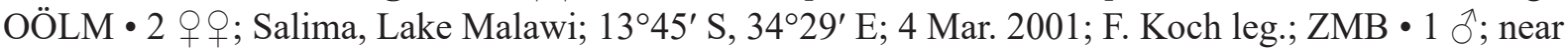
Sani, beach; 1301'53" S, 34¹9'47" E; 4-8 Mar. 2008; J. Oehlke leg.; coll. Jacobs. - Southern Region •

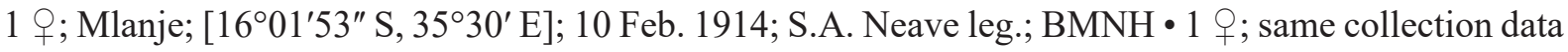
as for preceding but $13 \mathrm{Feb} .1914 ; \mathrm{BMNH}$.

MALI • 1 đ̂; inundation zone of Niger River, Koundé-Saré; 20 Aug. 1945; BMNH. - Mopti Region • $1 \partial^{7} ; 10$ km S of Mopti; [14²2'11.4" N, 408'56.3" W]; 20 Aug. 1991; M. Schwarz leg.; OÖLM.

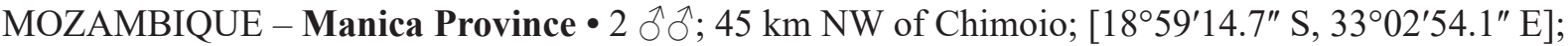

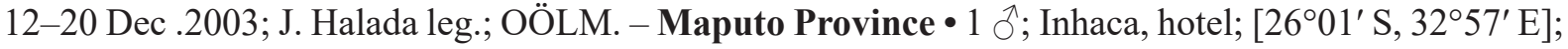

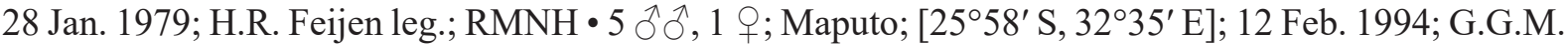

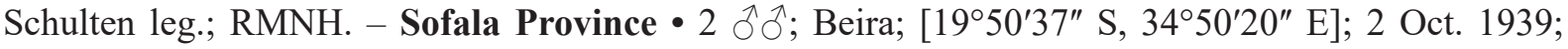
A.H.N. leg.; BMNH 3 ठึ; same locality as for preceding; 3 Oct. 1954; L.F. Brown leg.; BMNH.

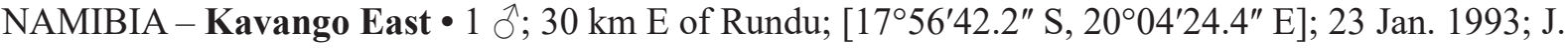
Gusenleitner leg.; OÖLM • 1 đ’, 4 우우 Kaudom-Camp; 18³1' S, 2043’ E; 22-25 Nov. 1992; F. Koch

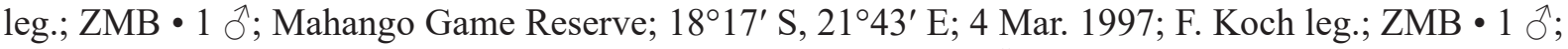

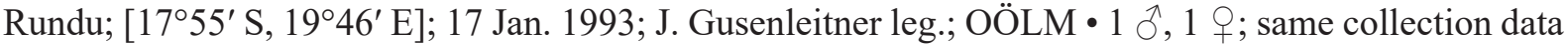
as for preceding but $19 \mathrm{Jan} .1993$; OÖLM $\bullet 1$; ; same collection data as for preceding but 22 Jan. 1993; OÖLM • 1 đ̂; same collection data as for preceding but 23 Jan. 1993; OÖLM • 1 क; same collection data as for preceding but $29 \mathrm{Jan}$. 1993; OÖLM • 1 ऽ; same collection data as for preceding but $30 \mathrm{Jan}$.

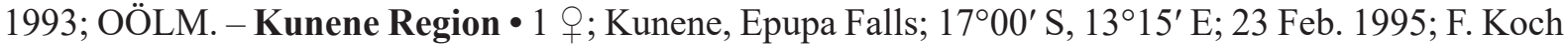

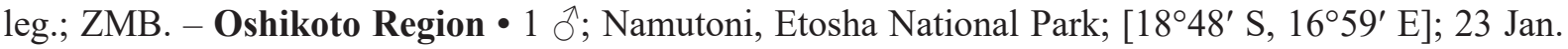
1993; F. Koch leg.; ZMB. - Otjozondjupa Region • 1 क; 100 km NE of Grootfontein; [18 $56^{\prime} 32.9^{\prime \prime}$ S,

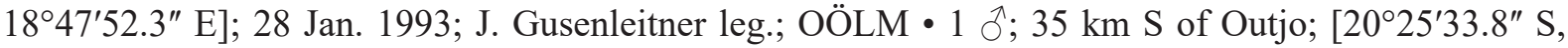
$\left.16^{\circ} 06^{\prime 2} 26.6^{\prime \prime} \mathrm{E}\right] ; 25$ Mar. 1979; J.G. Rozen leg.; AMNH • 1 q; Okahandja; [2159' S, 1655' E]; 2-4 Feb. $1972 ; \mathrm{BMNH}$.

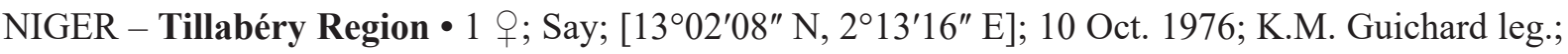
BMNH.

NIGERIA - Niger State • 1 क; Izom; [914'36" N, 700'26" E]; 11 Dec. 1958; R.W. Crosskey leg.;

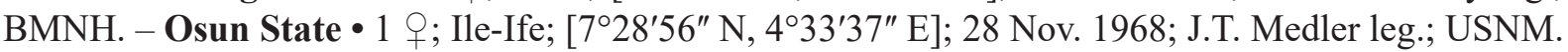

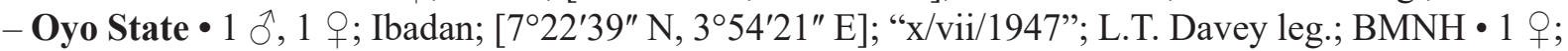
same collection data as for preceding; BMNH.

OMAN - Dhofar Governorate • 1 q; Raysut; [16 $57^{\prime}$ N, 5358'02" E]; 20 Aug. 1981; A.R. Pittaway leg.; BMNH.

REPUBLIC OF CÔTE D’IVOIRE - Gôh Region • 1 ' ; Gagnoa; [607'54" N, 557'02" E]; 2-4 Mar. 1984; M. Matthews leg.; BMNH. - Vallée du Bandama District • $4 \partial^{\lambda}{ }^{\lambda}, 1$ q ; Bouaké; [ $\left.7^{\circ} 41^{\prime} \mathrm{N}, 5^{\circ} 01^{\prime} \mathrm{W}\right]$; Jun. 1977; P.M. Elsen leg.; MRAC.

REPUBLIC OF THE CONGO • 1 đ̊; Route du Nord, km 17; 4 Nov. 1977; C. Morin leg.; MNHN. -

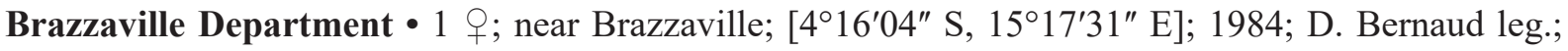
MNHN. - Kouilou Department • 1 क; Zambi; $6^{\circ}$ S, 12 $2^{\circ} 50^{\prime}$ E; 27 Jun. 1915; Lang and Chapin leg.; 
AMNH. - Pool Department • 1 क; Foulakori River near Kinkala; [4²1'41" S, 1445'52" E]; 18 Jan. 1973; J.F. Cornic leg.; MNHN • 1 क; Kintelé; [409' S, 15²0'32" E]; 9 Jan. 1977; G. Onore leg.; MNHN •

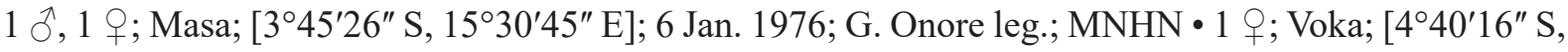
14²0'25" E]; 11 Nov. 1976; G. Onore leg.; MNHN.

SENEGAL • 1 क; Oct. 1976; G. Couturier leg.; MNHN. - Dakar Region • 1 q; Dakar; [1441'34" N, 17²6'48" W]; Sep. 1967; A. Villiers leg.; MNHN. - Fatick Region • 2 o \%; G. de Bandiala; [1339'40" N, 16³4'26" W]; 8-12 Dec. 1997; K.M. Guichard leg.; BMNH.

SOUTH AFRICA - Eastern Cape • 1 \%; $10 \mathrm{~km}$ SE of Alexandria, National Park; [33⒋'14.9" S,

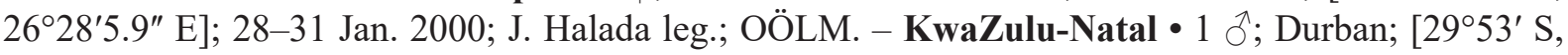

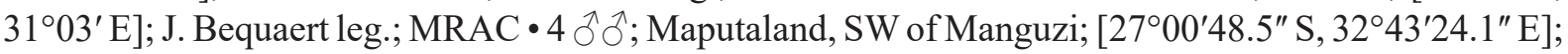

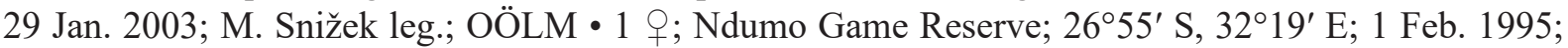

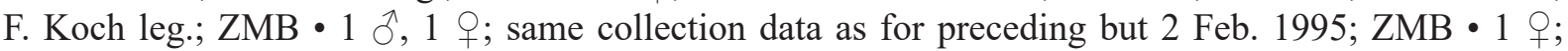

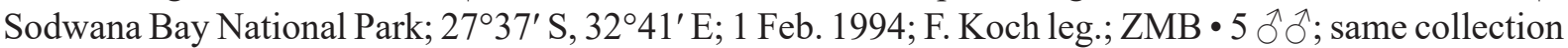
data as for preceding but $4 \mathrm{Feb} .1995$; ZMB $\bullet 1$ ग, 1 \% ; same collection data as for preceding but 25 Feb. 1997; ZMB • 1 J', 1 क ; St Lucia Estuary; [2822'58.8" S, 32²25'1.2" E]; 4-6 Dec. 1966; J.G. Rozen and D.J. Brothers leg.; AMNH • 1 ' ; St Lucia Park (Charter's Creek); $28^{\circ} 12^{\prime}$ S, 32 ${ }^{\circ} 25^{\prime}$ E; 7 Feb. 1995; F. Koch leg.; ZMB. - Limpopo • 3 우; 35 km W of Hoedspruit; 2423' S, 3040’ E; 19 Dec. 2009; J. Halada leg.; RMNH • 1 क; Farm 'Sericea”, Nylstroom [now Modimolle]; 244ㄱ' S, 28³9' E; 20-27 Mar. 2006; F. Koch leg.; ZMB • 1 q; Lekgalameetse Nature Reserve; $24^{\circ} 12^{\prime}$ S, $30^{\circ} 20^{\prime}$ E; 18 Dec. 1995;

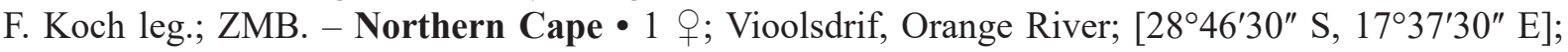
9 Jan. 1993; F. Koch leg.; THD-007-ZMB; GenBank CO1 gene: MW538580; GenBank EF-1 $\alpha$ gene: MW558245; ZMB. - Western Cape • 1 ō, 1 क ; Bontebok National Park, Swellendam; [ $34^{\circ} 04^{\prime} 06.8^{\prime \prime}$ S, $20^{\circ} 28^{\prime} 06.6^{\prime \prime}$ E]; 2-3 Jan. 1993; F. Koch leg.; ZMB • 1 +; Franschhoek; [3354'36.3" S, 1907'33.7" E]; 17 Mar. 1988; H. Malicky leg.; OÖLM.

SOUTH SUDAN - Greater Upper Nile Region • 1 q; Malakal; [9³2' N, 31³9'37" E]; 1 Dec. 1960; B. Hocking leg.; BMNH.

SUDAN - Al Qadarif • 1 क; Showak; $14.24^{\circ}$ N, 35.52 E; 6 Sep. 1946; C.E. Wilson leg.; BMNH.

TANZANIA • 1 §; Meru lowland; 1 Dec. 1905; Y. Sjöstedt leg.; NRS. - Dodoma Region • 1 ; 35 km E of Dodoma; 5 54' S, $35^{\circ} 45^{\prime}$ E; 14 Dec. 2006; J. Halada leg.; OÖLM • 1 ' $; 70 \mathrm{~km} \mathrm{~N}$ of Dodoma; $5^{\circ} 40^{\prime} \mathrm{S}$, $35^{\circ} 48^{\prime}$ E; 16 Dec. 2006; J. Halada leg.; OÖLM. - Iringa Region • 1 क ; $100 \mathrm{~km} \mathrm{NE}$ of Iringa; $7^{\circ} 37^{\prime} \mathrm{S}$, $36^{\circ} 17^{\prime}$ E; 9 Jan. 2007; J. Halada leg.; OÖLM • 1 đ’, 1 क; $50 \mathrm{~km} \mathrm{NW}$ of Iringa; $7^{\circ} 41^{\prime} \mathrm{S}, 35^{\circ} 22^{\prime}$ E; 7 Jan. 2007; J. Halada leg.; OÖLM. - Kigoma Region • 1 क; Kasoge, Mahali Peninsula; [609' S, 2946' E]; 20 Sep. 1959; BMNH. - Mjini Magharibi Region • 2 q $\circ$; Zanzibar; NRS • 1 ○े; Zanzibar, near M'nazi Moya; [609'42" S, 39¹1'28" E]; 20 Aug.-Sep. 1924; H.J. Snell leg.; BMNH. - Morogoro Region •

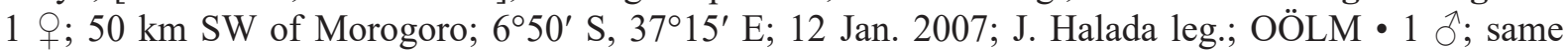
collection data as for preceding but M. Kadlecová leg.; OÖLM • 1 क ; NE of Mikumi, Camp; $7^{\circ} 23^{\prime 2} 24^{\prime \prime}$ S, 3701'45" E; 26-27 Feb. 2008; J. Oehlke leg.; coll. Jacobs. - Pwani Region • 1 क; 15 km E of Utete; $8^{\circ} 03^{\prime}$ S, 35 53' E; 16 Jan. 2007; J. Halada leg.; OÖLM • 1 क; $15 \mathrm{~km} \mathrm{~W}$ of Kibiti; $7^{\circ} 47^{\prime} \mathrm{S}, 38^{\circ} 49^{\prime} \mathrm{E}$;

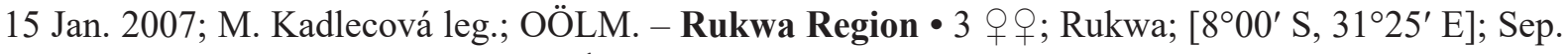

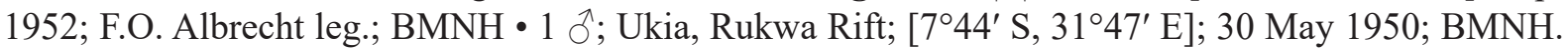

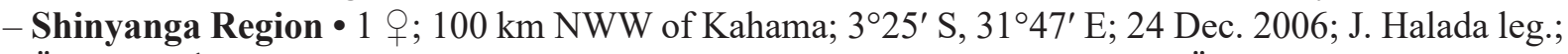
OÖLM • 1 ○ं; same collection data as for preceding but M. Kadlecová leg.; OÖLM. - Tanga Region •

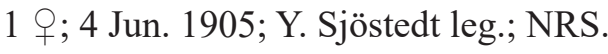

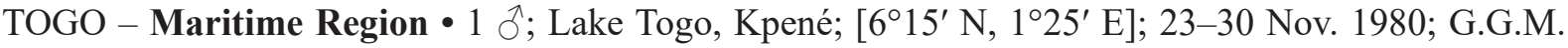
Schulten leg.; RMNH. - Savanes • 1 क; Sansanné-Mango; [10²1'20" N, 0²8'32" E]; 1896-1902; Thierry leg.; ZMB. 


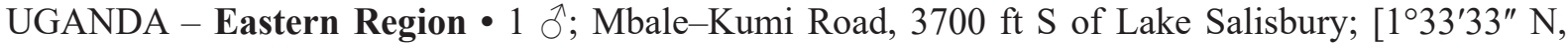

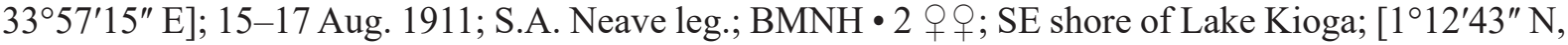

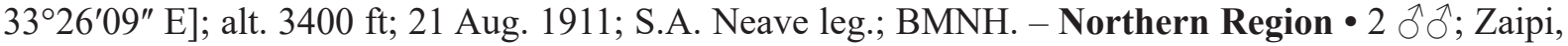
EMadi District; [3²4' N, 3159' E]; 2 Jun. 1927; G.D. Hale Carpenter leg.; OUMNH. - Western Region •

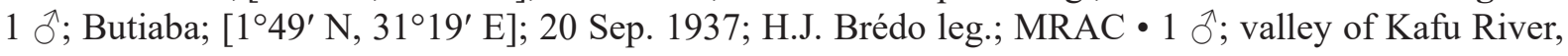
Unyoro; alt. 3400 ft; 23-28 Dec. 1911; S.A. Neave leg.; BMNH.

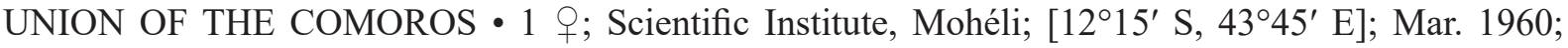

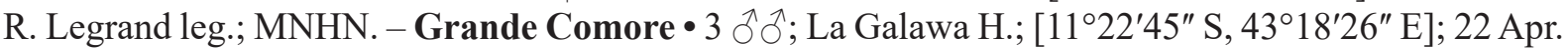

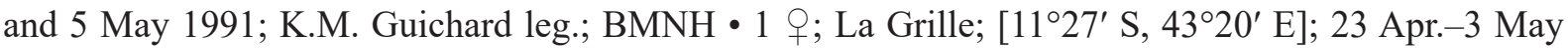
1991; K.M. Guichard leg.; BMNH.

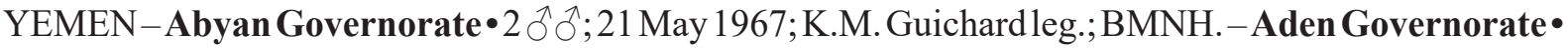
1 q; Aden; [12 $46^{\prime} 46^{\prime \prime} \mathrm{N}, 45^{\circ} 02^{\prime} 12^{\prime \prime}$ E]; 1930; E.B.C. Betts leg.; BMNH • 1 đ’; Al Huseini, near Lahej;

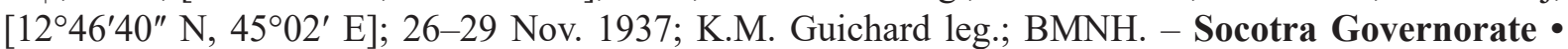

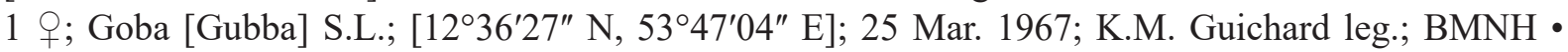

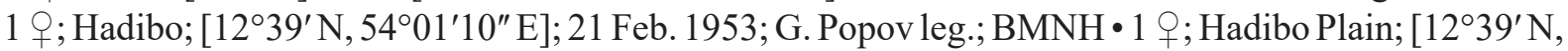

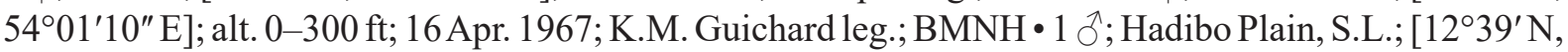
$54^{\circ} 01^{\prime} 10^{\prime \prime}$ E]; 19 Mar. 1967; K.M. Guichard leg.; BMNH・1 ${ }^{7}, 1$ q ; same collection data as for preceding but 7 Apr. 1967; BMNH.

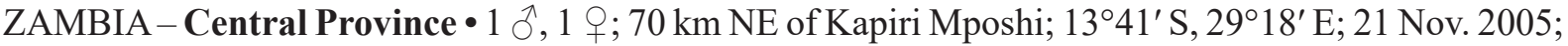

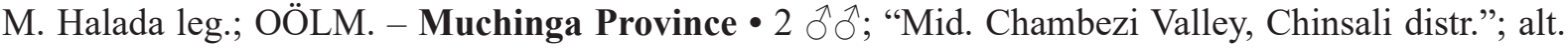
4000 ft; 20 Apr. 1908; S.A. Neave leg.; OUMNH. - Southern Province • 1 \&; 30 km W of Livingstone;

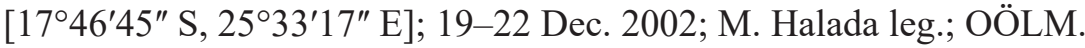

ZIMBABWE • 1 क; Victoria Falls; Feb. 1935; NRS. - Matabeleland North • 1 ; Hwange National Park, Dete; [18³7'08" S, 2651'33" E]; 11 Dec. 1997; J. Plass leg.; OÖLM.

\section{Description}

Female

Size. 25.1-32.0 mm.

COLOR. Black except for basal half of mandible, which is ferruginous. Cellular wing area hyaline, base of fore- and hindwing infuscate. Forewing with fuscous spot beyond marginal cell.

VeSTITURE. Appressed and erect setae on clypeus and paraocular area silvery. Appressed setae on collar both silvery and black, erect setae black, appressed and erect setae on scutum and propodeal enclosure black. Erect propodeal setae crimped, oriented perpendicularly. Clypeus without glabrous stripe. Scutellum densely and finely pubescent.

STRUCTURE. Free clypeal margin with short, broad, notched tooth, not stepped medially. Clypeus without indentation or carina. Scutellum convex. Metanotum raised, notably bituberculate. $2^{\text {nd }}$ recurrent vein joins interstitium between submarginal cells II and III. Propodeal enclosure without any notable ridges. Foretarsomere I 2.3-2.6 $\times$ length of antepenultimate spine. Petiole length $2.0-2.1 \times$ its medial width.

\section{Male}

SizE. 17.4-26.2 mm.

CoLOR. Black. Cellular wing area hyaline, base of fore- and hindwing infuscate. Forewing with fuscous spot beyond marginal cell. 


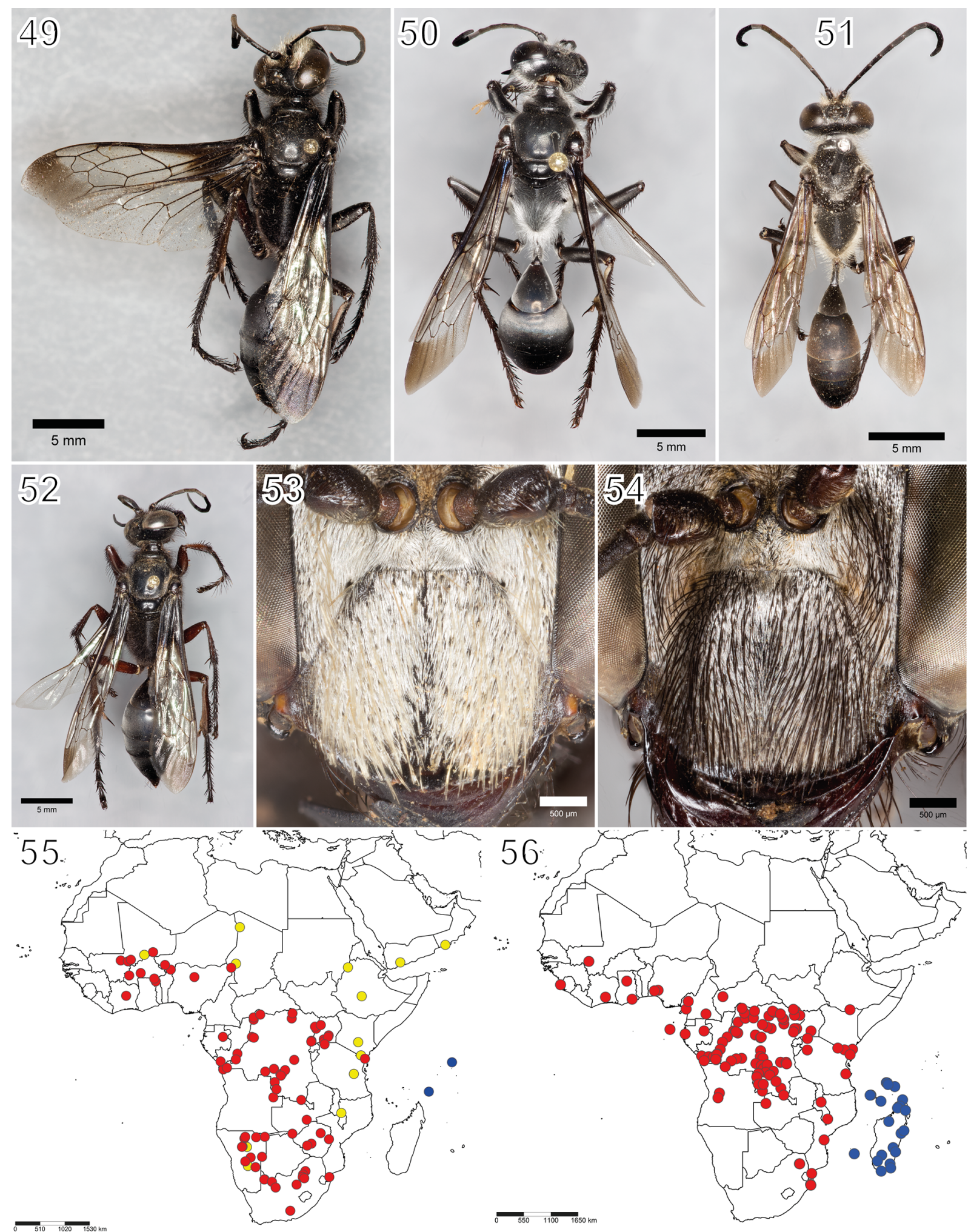

Figs 49-56. 49. Sphex fumicatus Christ, 1791, habitus of $q$. 50. S. lanatus Mocsáry, 1883, habitus of $q$. 51. S. rufinervis Pérez, 1895, habitus of $\widehat{O}$. 52. S. taschenbergi Magretti, 1884, habitus of q. $^{2}$ 53. S. fumicatus, frontal view of $q$. 54. S. taschenbergi, frontal view of $q$. 55. Geographic distribution of S. lanatus (red), S. rufinervis (blue) and S. taschenbergi (yellow). 56. Geographic distribution of S. tomentosus Fabricius, 1787 (red) and S. torridus F. Smith, 1873 (blue). 
VestiTURE. Appressed and erect setae on clypeus and paraocular area silvery. Appressed setae on collar both silvery and black, erect setae black, appressed and erect setae on scutum and propodeal enclosure black. Erect propodeal setae crimped, oriented perpendicularly. Clypeus without glabrous stripe. Scutellum densely and finely pubescent.

Structure. Free clypeal margin simple. Scutellum slightly convex. Metanotum raised, notably bituberculate. $2^{\text {nd }}$ recurrent vein joins interstitium between submarginal cells II and III. Propodeal enclosure without any notable ridges. Posterior margin of metasomal tergum VII convex. Posterior margin of metasomal sternum VII simple, of metasomal sternum VIII triangular. Penis valvae without conspicuous modifications. Petiole length $2.2-2.3 \times$ its medial width. Flagellomeres V + VI with broad placoids, covering entire length of flagellomere $\mathrm{V}$ and proximal four-fifths of flagellomere VI.

\section{Variation}

In some specimens, the legs are dark ferruginous to a varying degree.

\section{Distribution}

Very widespread in Africa; also occurs in the eastern Mediterranean region.

\section{Remarks}

Despite not having examined type material, we are certain that our interpretation of S. fumicatus is correct. The species occurs all over the African continent and is found on the Arabian Peninsula as well. It is one of, if not the most commonly collected Sphex from the Afrotropical region. Its diagnostic characters are easy to see and therefore commonly included in descriptions, which makes synonymization possible even for works from authors that are not especially knowledgeable about this genus.

Sphex lanatus Mocsáry, 1883

Figs 50, 55 (red)

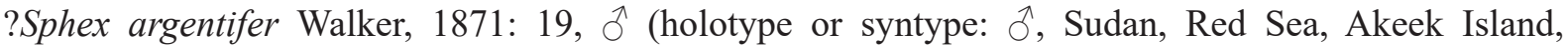
destroyed). Synonymized with Sphex umbrosus by Kohl, 1890: 407.

Sphex lanatus Mocsáry, 1883: 34, §̂ (holotype: ${ }_{0}$, South Africa, “Transvaal”, TMB, not examined).

\section{Differential diagnosis}

Within the argentatus group, S. lanatus is characterized through its entirely silvery vestiture. Females (Fig. 50) can be distinguished from those of S. feijeni nom. nov. (Fig. 43) through having the propodeal sculpture largely obscured by appressed setae, whereas the propodeal vestiture is more sparse in that species and leaves 7-10 distinct transversal ridges visible that are absent in $S$. lanatus. Females of S. erythrinus are distinguishable through their yellow-tinged wings (Fig. 41).

Males of $S$. lanatus are recognizable by their longer and more slender petiole, the length of which is greater than 2.7 times its medial width. This coefficient is notably smaller than 2.4 in males of S. erythrinus (Fig. 42) and S. feijeni nom. nov. (Fig. 44). Furthermore, S. erythrinus and S. feijeni nom. nov. have the placoids covering at least flagellomeres III-V, whereas $S$. lanatus has only a single placoid on flagellomere V. Finally, S. rufinervis is similar (Fig. 51), but its erect clypeal setae are partially or completely black in both sexes.

\section{Material examined}

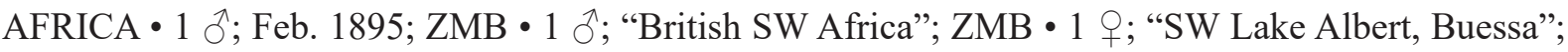
12 Aug. 1891; ZMB • 1 o; “Tanganyika”; 1936; H.A. Lindeman leg.; BMNH. 
BOTSWANA • 1 đิ, 1 क; “Kalahari desert, Sevorelela Kokot”; Oct.-Nov. 1904; L. Schultze leg.; ZMB.

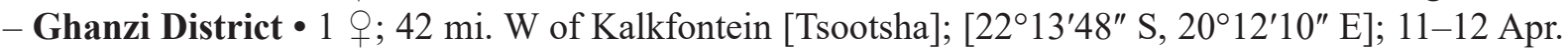
1972; BMNH・ 1 o, 1 o ; Mongalatsela; 8 May 1924; J. Maurice leg.; BMNH.

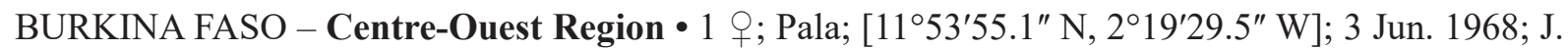
Hamon leg.; MNHN • 1 क; same collection data as for preceding but 8 Jun. 1968; MNHN. - Hauts-

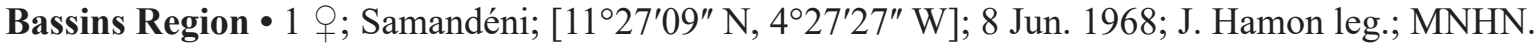

CAMEROON • 1 万’; "Neu-Kamerun"; G. Tessmann leg.; ZMB. - Far North Region • 1 क; Lake Chad; [1253'38.9" N, 14²9'19.4" E]; 6-12 Aug. 1909; Riggenbach leg.; ZMB.

DEMOCRATIC REPUBLIC OF THE CONGO • 1 क; "Tanganika"; Hecq leg.; MRAC. - Bas-Uele • 1 \%; Bambesa; [ $3^{\circ} 28^{\prime}$ N, $25^{\circ} 43^{\prime}$ E]; 20 Sep. 1933; H.J. Brédo leg.; MRAC • 1 \%; same locality as for preceding; 8 Jul. 1937; J. Vrydagh leg.; MRAC • 1 क; Tukpwo; [4²5'56" N, 2550'50" E]; Jul. 1937; J. Vrydagh leg.; MRAC • 1 9 ; same collection data as for preceding but Aug. 1937; MRAC. - Haut-

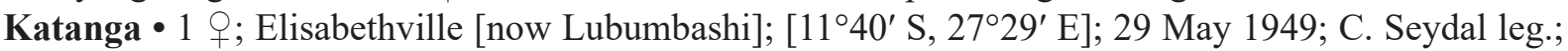
USNM - 1 \%; same collection data as for preceding but May 1949; MRAC. - Ituri Province $\bullet 1$; Bunia; [1 $\left.{ }^{\circ} 34^{\prime} \mathrm{N}, 30^{\circ} 15^{\prime} \mathrm{E}\right]$; 15 Aug. 1934; J.V. Leroy leg.; MRAC • 1 क; Mahagi-Niarembe; [2॰14'49" N,

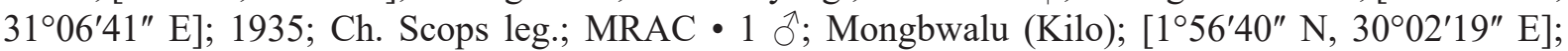
1939; Scheitz leg.; MRAC. - Kasaï • 1 q; Tshikapa; [6²4' S, 20²4' E]; Mar. 1939; Mevr. Bequaert leg.; MRAC • 1 ; same collection data as for preceding but Apr. 1939; MRAC. - Kasaï-Central •

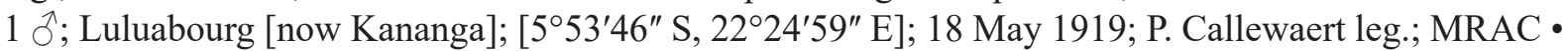

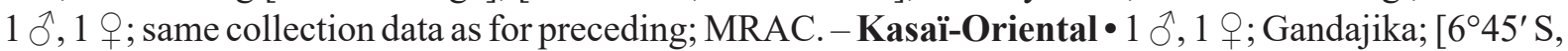
$23^{\circ} 57^{\prime}$ E]; 1956; P. de Francquen leg.; MRAC $11{ }^{\lambda}$; same locality as for preceding; Dec. 1925; Ch. Seydel leg.; MRAC • 1 đ; same locality as for preceding; Ch. Seydel leg.; MRAC. - Kongo Central • $1 \mathrm{O}^{\lambda}$; Boma; $6^{\circ} 00^{\prime} \mathrm{S}, 13^{\circ} 00^{\prime} \mathrm{E}$; 18 Jun. 1915; Lang and Chapin leg.; AMNH • 1 ô; same collection data

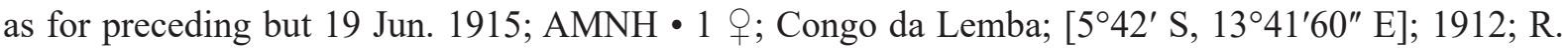
Mayné leg.; MRAC • 1 q; Mayumbe; [ $\left.4^{\circ} 30^{\prime} \mathrm{S}, 12^{\circ} 30^{\prime} \mathrm{E}\right]$; R. Verschueren leg.; MRAC. - Lomami •

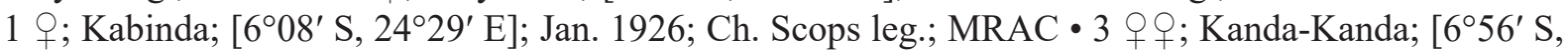

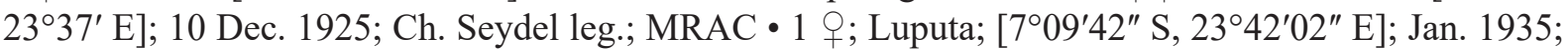

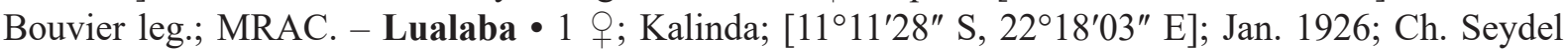
leg.; MRAC • 1 q; Kapanga; [ $8^{\circ} 21^{\prime}$ S, $22^{\circ} 34^{\prime}$ E]; Dec. 1932; F.G. Overlaet leg.; MRAC • 1 \%; same

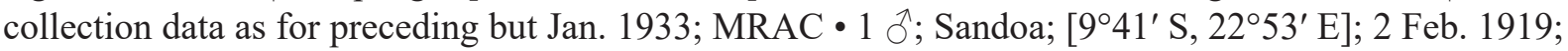
F.G. Overlaet leg.; MRAC - 1 क; same collection data as for preceding but 20 Mar. 1919; MRAC. -

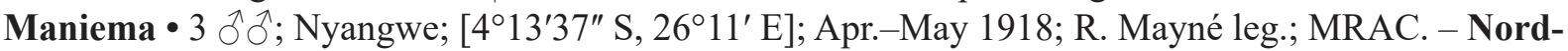

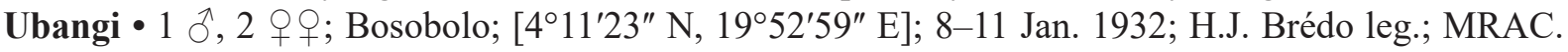

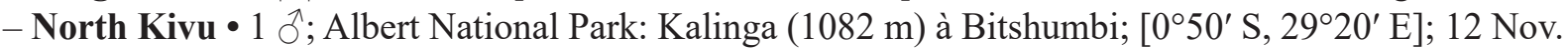
1934; G.F. de Witte leg.; MRAC • 1 ơ; Vitschumbi, SW of Lake Edward; [041' S, 29²3' E]; 2 May 1901; ZMB. - Province of Équateur • 1 q; Coquilhatville [now Mbandaka]; [0 03' N, 12 ${ }^{\circ} 57^{\prime} \mathrm{E}$ ]; 10

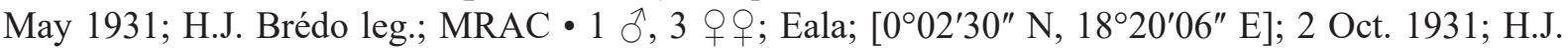

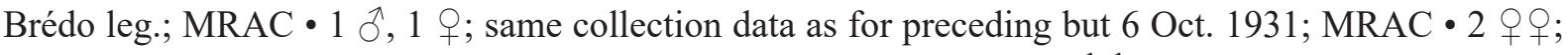

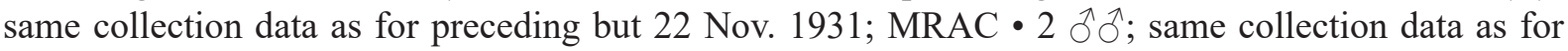
preceding but Mar. 1932; MRAC 11 ते; same collection data as for preceding but May 1932; MRAC • 1 ; ; same locality as for preceding; May 1935; J. Ghesquière leg.; MRAC. - Sud-Ubangi • 1 đ; Libenge; [339'11" N, 18³8'08" E]; 10 Dec. 1931; H.J. Brédo leg.; MRAC • 1 ơ; same locality as for

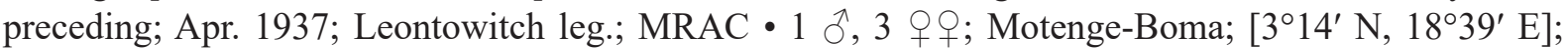

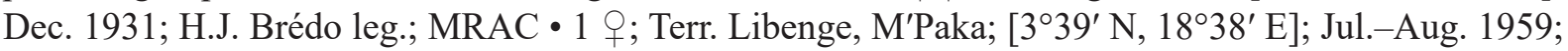
M. Pecheur leg.; MRAC. - Tshuapa • 1 q; Bokuma; [006' S, 1841' E]; Jul. 1952; R.P. Lootens leg.; MRAC. 
KENYA - Kwale County • 1 q; Kwali Forest, 20 mi. W of Mombasa; [400'34" S, 39¹8'18" E]; 1 Jun. 1948; M. Steele leg.; BMNH.

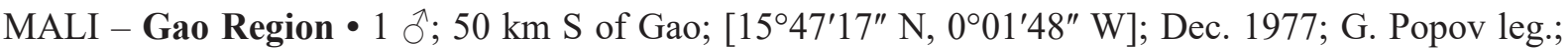

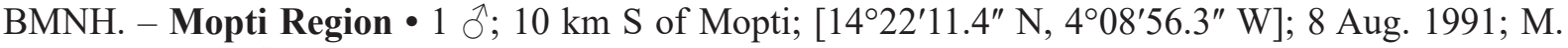

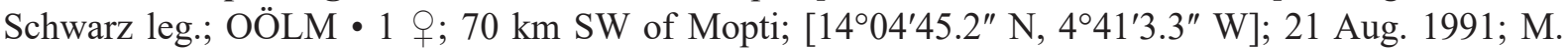

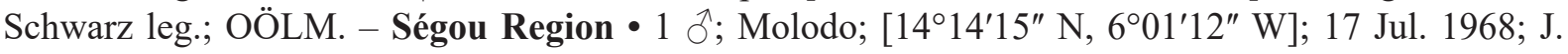
Hamon leg.; MNHN.

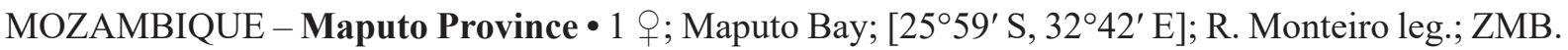

NAMIBIA • 1 क; "German SW Africa"; 1901; Lübbert leg.; ZMB. - Hardap Region • 1 ô; 23 km

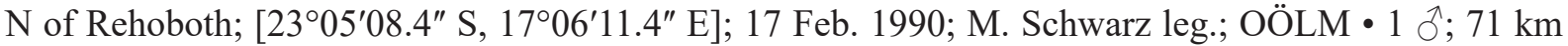
E of Stampriet; $24^{\circ} 09^{\prime}$ S, $1^{\circ} 00^{\prime}$ E; 27 Mar. 2000; F.W. and S.K. Gess leg.; AMG. - Kavango East •

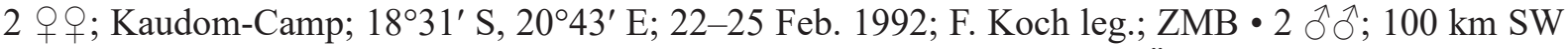
of Rundu; [18³5'48" S, 1906'58.3" E]; 1 Feb. 1993; M. Schwarz leg.; OÖLM. - Khomas Region •

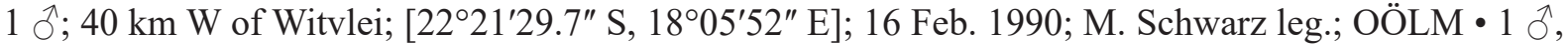
1 q; S of Okasewa; 22 ${ }^{\circ} 34^{\prime} 53^{\prime \prime}$ S, $18^{\circ} 11^{\prime} 15^{\prime \prime}$ E; 12-17 Feb. 2010; J. Oehlke leg.; coll. Jacobs. - Oshikoto

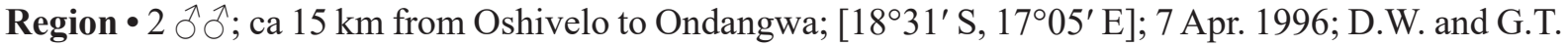
Gess leg.; AMG • 2 + O ; Namutoni, Etosha National Park; 1848' S, 1656' E; 27 Feb. 1994; F. Koch leg.; THD-005-ZMB; GenBank EF-1 $\alpha$ gene: MW558243; GenBank LWR gene: MW582281; ZMB. -

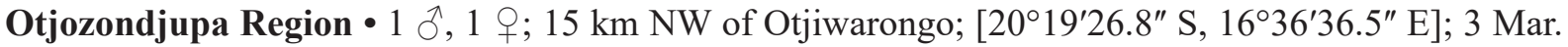
1990; M. Schwarz leg.; OÖLM • 1 क; 15-20 km NW of Otjiwarongo; [20²3'02" S, 16²8'54" E]; 3 Mar. 1990; W.J. Pulawski leg.; CAS.

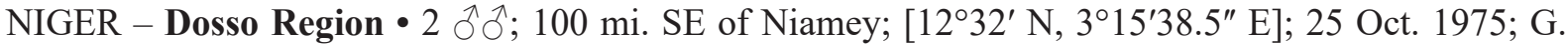

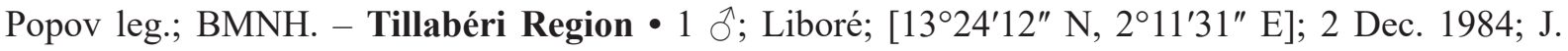
Hamon leg.; MNHN.

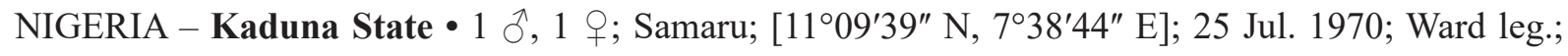
BMNH.

REPUBLIC OF CÔTE D’IVOIRE - Vallée du Bandama District • 1 \%; Bouaké; [741' N, $5^{\circ} 01^{\prime} \mathrm{W}$; Jul. 1977; P.M. Elsen leg.; MRAC.

REPUBLIC OF THE CONGO - Pool Department • 1 + ; Inoni; [ $\left.3^{\circ} 04^{\prime} 5.8^{\prime \prime} \mathrm{S}, 15^{\circ} 38^{\prime} 10.8^{\prime \prime} \mathrm{E}\right]$; 15 Nov.

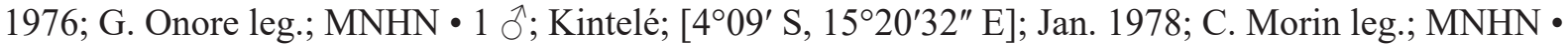
1 ; ; same collection data as for preceding but 3 Apr. 1978; MNHN • 1 ; ; same locality as for preceding; Mar. 1978; G. Onore leg.; MNHN.

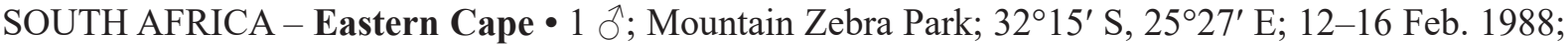

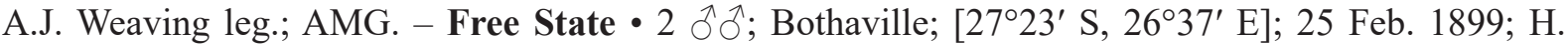
Brauns leg.; TMP $\bullet 1$; ; same collection data as for preceding; TMP $\bullet 1$; same collection data as for

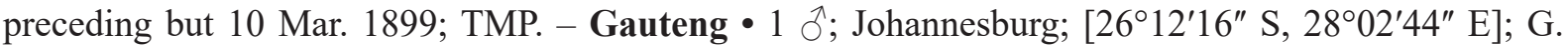

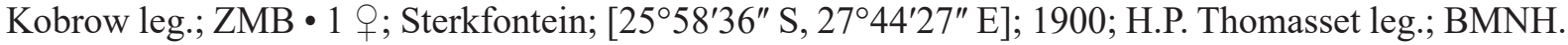

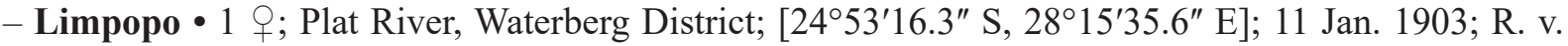
Jutrzencka leg.; TMP• 1 ते; Waterberg District; 1898-1899; R. v. Jutrzencka leg.; TMP. - North West •

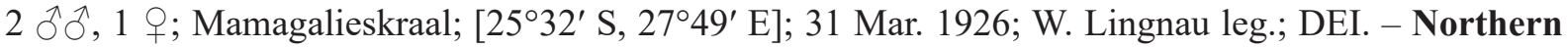

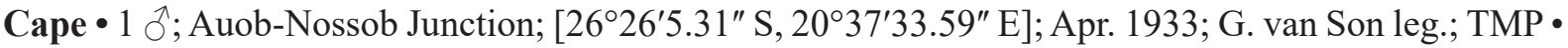

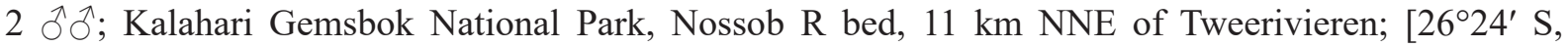

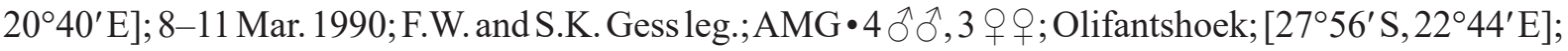


24 Mar. 1990; M. Schwarz leg.; OÖLM • 1 ô; S of Kalahari Desert, Van Zylsrus; [2652'1.2" S, 22 03' E]; 15 Jan. 2001; M. Snižek leg.; THD-021-OOLM; GenBank CO1 gene: MW538579; OÖLM • $8 \widehat{\jmath}, 1$ q ; same collection data as for preceding; OÖLM.

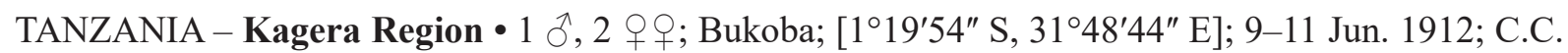
Gowdey leg.; BMNH.

TOGO - Savanes • 1 q; “Tapuog” [Dapaong?]; [1052' N, 0¹2' E]; Aug. 1901; Thierry leg.; ZMB • 1 ; ; Sansanné-Mango; [10²1'20" N, 0²8'32" E]; Thierry leg.; ZMB.

UGANDA - Central Region • 1 \%; Buddu, NW shores of Victoria Nyanza; [0 $0^{\circ} 25^{\prime} \mathrm{S}, 31^{\circ} 40^{\prime} \mathrm{E}$ ]; $12-15$ Sep. 1911; S.A. Neave leg.; BMNH • 1 q; Entebbe; [003' N, 32²7'36" E]; 6-10 May 1912; C.C. Gowdey leg.; BMNH • 2 o 9 ; same collection data as for preceding but Aug. 1912; BMNH 1 o';

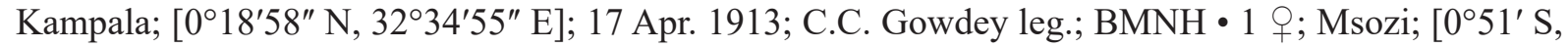

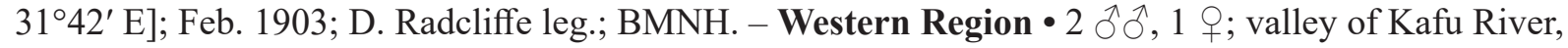
Unyoro; alt. 3400 ft; 23-28 Dec. 1911; S.A. Neave leg.; BMNH.

ZAMBIA - Lusaka Province • 1 q; 25 km E of Lusaka; 15²1' S, 28³0' E; 3 Mar. 1995; W.J. Pulawski leg.; CAS. - Northern Province • 1 đ̃; "L. Chambezi V., Kasama distr."; alt. 3900 ft; 4-6 May 1908; S.A. Neave leg.; OUMNH.

ZIMBABWE - Bulawayo • 1 क; Bulawayo; [2009' S, 28³5' E]; Dec. 1903; G.A.K. Marshall leg.;

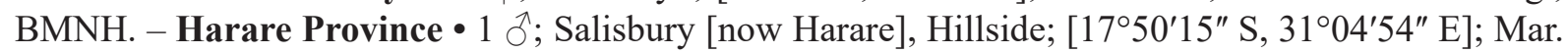
1926; H.S. Leeson leg.; BMNH. - Manicaland • 1 गे; Umtali [now Mutare]; [18 ${ }^{\circ} 58^{\prime} \mathrm{S}, 32^{\circ} 38^{\prime}$ E]; A. Bodong leg.; DEI. - Matabeleland North • 1 ơ; Victoria Falls; $17^{\circ} 56^{\prime} \mathrm{S}, 25^{\circ} 50^{\prime}$ E; 28-31 Mar. 1998;

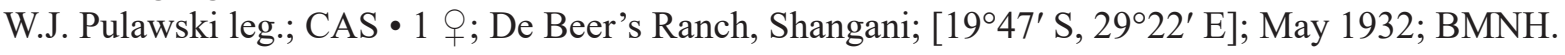

\section{Description}

Female

Size. $21.2-33.3 \mathrm{~mm}$.

COLOR. Black except for the following, which are dark ferruginous: basal half of mandible and legs from trochanter onward, especially femur and tibia of mid- and hindlegs. Cellular wing area hyaline, base of fore- and hindwing infuscate, apical margin of forewing fuscous.

VeSTITURE. Appressed and erect setae on clypeus, paraocular area, collar, scutum and propodeal enclosure silvery. Erect propodeal setae crimped, oriented anteriorly. Clypeus without glabrous stripe. Scutellum densely and finely pubescent.

STRUCTURE. Free clypeal margin simple, not stepped medially. Clypeus without indentation or carina. Scutellum convex. Metanotum raised, notably bituberculate. $2^{\text {nd }}$ recurrent vein joins markedly proximal from interstitium between submarginal cells II and III. Propodeal enclosure without any notable ridges. Foretarsomere I 2.7-2.8 $\times$ length of antepenultimate spine. Petiole length $2.3-2.5 \times$ its medial width.

Male

Size. $17.6-23.8 \mathrm{~mm}$.

COLOR. Black except for the following, which are dark ferruginous: stripe in center of mandible, hindtibia and often part of hindfemur. Cellular wing area hyaline, base of fore- and hindwing infuscate, forewing with fuscous spot beyond marginal cell. 
VeSTITURE. Appressed setae on clypeus, paraocular area, collar, scutum and propodeal enclosure silvery. Erect setae on clypeus and paraocular area silvery intermixed with black ones, exclusively silvery on collar, scutum and propodeal enclosure. Erect propodeal setae crimped, oriented anteriorly. Clypeus without glabrous stripe. Scutellum densely and finely pubescent.

StruCture. Free clypeal margin simple. Scutellum convex. Metanotum raised, notably bituberculate. $2^{\text {nd }}$ recurrent vein joins markedly proximal from interstitium between submarginal cells II and III. Posterior margin of metasomal tergum VII convex. Posterior margin of metasomal sternum VII simple, of metasomal sternum VIII very slightly concavely emarginate. Penis valvae without conspicuos modifications. Petiole length $2.8-3.0 \times$ its medial width. Flagellomere V with narrow placoid covering its proximal two-thirds.

\section{Variation}

Unknown.

\section{Distribution}

Widepsread in Africa.

\section{Sphex rufinervis Pérez, 1895}

Figs 51, 55 (blue)

Sphex rufinervis Perez, 1895: 209, §̂,, .

\section{Differential diagnosis}

This species (Fig. 51) is one of four within this group that have uniformly silvery propodeal setae. An easily visible character that distinguishes it from the other three, S. erythrinus (Figs 41-42), S. feijeni nom. nov. (Figs 43-44) and S. lanatus (Fig. 50), are the largely or completely black erect clypeal setae in both sexes of $S$. rufinervis. The other species mentioned have them uniformly silvery.

\section{Material examined}

\section{Lectotype}

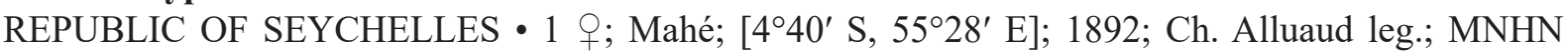
(designated by Menke in Bohart \& Menke 1976: 116).

\section{Paralectotypes}

REPUBLIC OF SEYCHELLES • 1 $\curvearrowright, 3$, $\odot$; same collection data as for lectotype; MNHN.

\section{Other material}

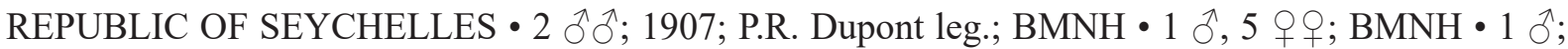
same collection data as for lectotype; MNHN $\bullet 5 \hat{\jmath} \hat{\jmath}, 1$; ; same collection data as for lectotype but A.

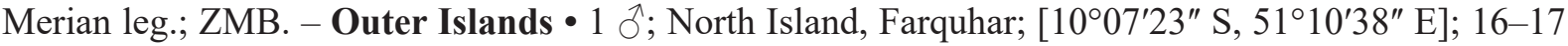
Nov. 1952; E.S. Brown leg.; BMNH.

\section{Description}

\section{Female}

SizE. $23.4 \mathrm{~mm}$.

Color. Black except for ferruginous stripe in center of mandible. Cellular wing area hyaline, apical margin of forewing fuscous. 
VeSTITURE. Appressed setae on clypeus, paraocular area, collar, scutum and propodeal enclosure silvery. Erect setae on clypeus black, on paraocular area black intermixed with silvery ones, on collar silvery intermixed with some black ones, on scutum and propodeal enclosure exclusively silvery. Erect propodeal setae oriented anteriorly. Clypeus without glabrous stripe. Scutellum densely and finely pubescent.

STRUCTURE. Free clypeal margin with two small teeth, not stepped medially. Clypeus without indentation or carina. Scutellum convex. Metanotum raised, slightly bituberculate. $2^{\text {nd }}$ recurrent vein joins markedly proximal from interstitium between submarginal cells II and III. Propodeal enclosure without any notable ridges. Foretarsomere I 2.0-2.3 $\times$ length of antepenultimate spine. Petiole length $2.0 \times$ its medial width.

\section{Male}

SizE. $19.4-21.7 \mathrm{~mm}$.

CoLoR. Black, except for ferruginous stripe in center of mandible. Cellular wing area hyaline, forewing with fuscous spot beyond marginal cell.

VeSTITURE. Appressed setae on clypeus, paraocular area, collar, scutum and propodeal enclosure silvery. Erect setae on clypeus black and usually intermixed with silvery ones, on paraocular area silvery and usually intermixed with some black ones, exclusively silvery on collar, scutum and propodeal enclosure. Erect propodeal setae oriented anteriorly. Clypeus without glabrous stripe. Scutellum densely and finely pubescent.

StRUCTURE. Free clypeal margin simple. Scutellum convex. Metanotum slightly raised, slightly bituberculate. $2^{\text {nd }}$ recurrent vein joins markedly proximal from interstitium between submarginal cells II and III. Posterior margin of metasomal tergum VII convex. Posterior margin of metasomal sternum VII simple, of metasomal sternum VIII concavely emarginate. Penis valvae without conspicuous modifications. Petiole length $2.4 \times$ its medial width. Flagellomere $\mathrm{V}$ with narrow placoid covering its proximal four-fifths.

\section{Variation}

Unknown.

\section{Distribution}

Seychelles.

Sphex taschenbergi Magretti, 1884

Figs 52, 54, 55 (yellow)

Sphex taschenbergi Magretti, 1884: 581, + (syntypes: Ethiopia, Amhara Region, Metemma, [12 $\left.57^{\prime} 17^{\prime \prime} \mathrm{N}, 36^{\circ} 09^{\prime} 19^{\prime \prime} \mathrm{E}\right], \mathrm{MSNG}$, not examined).

\section{Differential diagnosis}

This species (Fig. 52) shares many characteristics with S. fumicatus, like black erect propodeal setae and basally infuscate but otherwise hyaline forewings. Among the African argentatus group members, this combination is found only in those two. From S. fumicatus, S. taschenbergi is distinguished through having uniformly black erect clypeal setae (Fig. 54) instead of silvery ones (Fig. 53) in both sexes.

\section{Material examined}

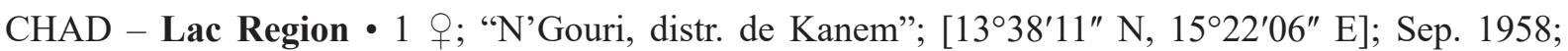

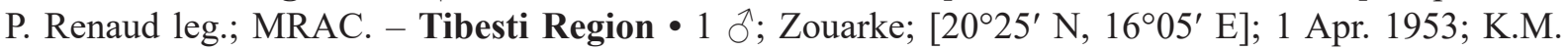
Guichard leg.; BMNH. 
ETHIOPIA - Oromia Region • 2 क $q$; Lake Langano; [7³6' N, 3843' E]; 29-30 Apr. 2007; J. Halada leg.; OÖLM.

KENYA - Kitui County • 1 क ; E of Mwingi; [0 $\left.0^{\circ} 56^{\prime} \mathrm{S}, 38^{\circ} 03^{\prime} \mathrm{E}\right]$; 14 May 2007; M. Halada leg.; OÖLM.

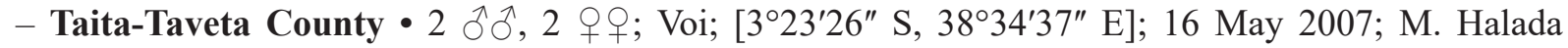
leg.; OÖLM • 1 क; near Voi (Tsavo); [3²3'26" S, 38³4'37" E]; 8-18 Nov. 1996; Mi. Halada leg.; OÖLM • 1 \&; same collection data as for preceding but 22 Nov.-2 Dec. 1996; OÖLM・1 §’; Voi River; [3²3'26" S, 38³4'37" E]; 14 Apr. 2007; M. Halada leg.; OÖLM.

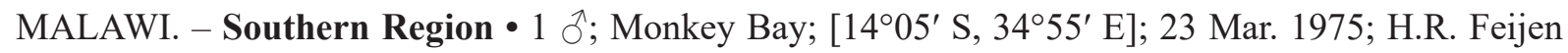
leg.; RMNH.

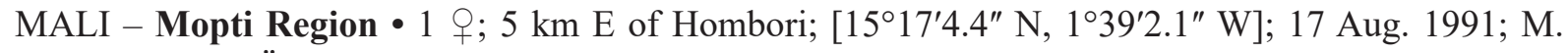
Schwarz leg.; OÖLM.

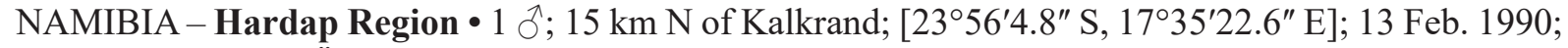
M. Schwarz leg.; OÖLM. - Otjozondjupa Region • 1 o; Waterberg, Okatijikona; 20²4' S, $1^{\circ} 24^{\prime}$ E; 27-28 Feb. 2010; F. Koch leg.; THD-004-ZMB; GenBank CO1 gene: MW538576; GenBank EF-1 $\alpha$ gene: MW558244; GenBank LWR gene: MW582283; ZMB • 1 q ; same collection data as for preceding but 13-14 Mar. 2010; ZMB.

OMAN • 1 §; Barelmi; Feb.-Mar. 1943; D.V. Fitzgerald leg.; BMNH. - Dhofar Governorate • 1 ठै; "Aim Arzat" [Ayn Razat]; [1707'47" N, 5414'11" E]; 18 Aug. 1981; A.R. Pittaway leg.; BMNH.

SUDAN - Khartoum State • 2 ふぶ; Omdurman; 4 Apr. 1901; NRS.

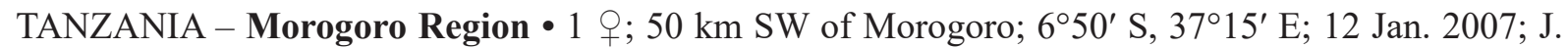
Halada leg.; OÖLM.

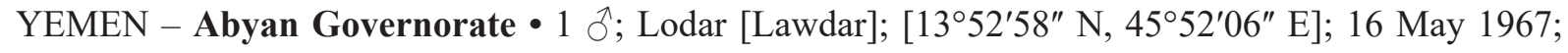
K.M. Guichard leg.; BMNH.

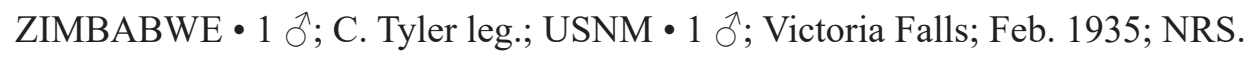

\section{Description}

Female

Size. $22.7-28.1 \mathrm{~mm}$.

CoLOR. Black, femora and tibia dark ferruginous. Cellular wing area hyaline, base of fore- and hindwing infuscate. Forewing with fuscous spot beyond marginal cell.

VeSTITURE. Appressed setae on clypeus and paraocular area silvery, on collar, scutum and propodeal enclosure black. Erect setae on clypeus, paraocular area, collar, scutum and propodeal enclosure black. Erect propodeal setae crimped, oriented perpendicularly. Clypeus without glabrous stripe. Scutellum densely and finely pubescent.

STRUCTURE. Free clypeal margin simple, not stepped medially. Clypeus without indentation and carina. Scutellum convex. Metanotum raised, notably bituberculate. $2^{\text {nd }}$ recurrent vein joins markedly proximal from interstitium between submarginal cells II and III. Propodeal enclosure without any notable ridges. Foretarsomere I 2.1-2.3 $\times$ length of antepenultimate spine. Petiole length $1.8-2.1 \times$ its medial width.

Male

Size. $18.9-22.6 \mathrm{~mm}$. 
CoLOR. Black. Cellular wing area hyaline, base of fore- and hindwing infuscate. Forewing with fuscous spot beyond marginal cell.

VESTITURE. Appressed setae on clypeus, paraocular area and collar silvery, on scutum and propodeal enclosure black. Erect setae on clypeus, paraocular area, collar, scutum and propodeal enclosure black. Erect propodeal setae crimped, oriented perpendicularly. Clypeus without glabrous stripe. Scutellum densely and finely pubescent.

STRUCTURE. Free clypeal margin simple. Scutellum convex. Metanotum raised, notably bituberculate. $2^{\text {nd }}$ recurrent vein joins markedly proximal from interstitium between submarginal cells II and III. Propodeal enclosure without any notable ridges. Posterior margin of metasomal tergum VII convex. Posterior margin of metasomal sternum VII simple, of metasomal sternum VIII triangular. Penis valvae without conspicuous modifications. Petiole length $2.1-2.3 \times$ its medial width. Flagellomere II with or without broad placoid covering its proximal two-thirds, flagellomeres III-VI with broad placoid covering their entire length.

\section{Variation}

In some specimens, the legs are dark ferruginous to a varying degree.

\section{Distribution}

Chad, Ethiopia, Kenya, Mali, Namibia, Oman, Sudan, Tanzania, Yemen, Zimbabwe.

Sphex tomentosus Fabricius, 1787

Figs 56 (red), 57, 60-61

Sphex tomentosus Fabricius, 1787: 274 (holotype: đ, Sierra Leone, Pflug leg., ZMUC, not examined). Sphex tuberculatus F. Smith, 1873: 291, +. Junior primary homonym of Sphex tuberculatus de Villers, 1789 (now in Cerceris Latreille, 1802). Synonymized with Sphex tomentosus by van der Vecht 1961: 29.

\section{Differential diagnosis}

This species (Fig. 57) can easily be distinguished from other members of the argentatus group through the uniformly rich golden facial setae present in both sexes. Males also possess a distinct edge on tergum VII (Fig. 60) that is unique among all African Sphex, as well as conspicuously heart-shaped valvicepes (Figs 60-61).

\section{Material examined}

AFRICA • 1 \%; Jan. 1891; NRS • 2 q ; Région des Lacs; Sagona leg.; MRAC.

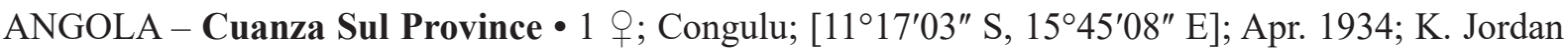

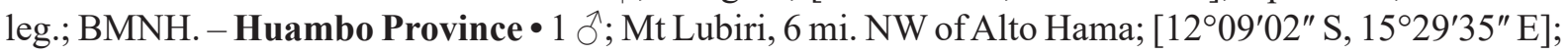
7 Mar. 1972; BMNH.

CAMEROON - Adamawa Region 1 1 ; SE of Ngaoundéré, ranch; $\left[7^{\circ} 08^{\prime} \mathrm{N}, 13^{\circ} 42^{\prime} \mathrm{E}\right]$; 9-12 May 2006; J. Oehlke leg.; coll. Jacobs. - East Province • 1 O ; D'Ja Posten; $3.15^{\circ} \mathrm{N}, 13.30^{\circ} \mathrm{E}$; $15 \mathrm{May}-1 \mathrm{Jul}$. 1936; F.G. Merfield leg.; BMNH. - Littoral Region • 2 o 9 ; Douala; [ $4^{\circ} 03^{\prime}$ N, $9^{\circ} 41^{\prime}$ E]; v. Rothkirch leg.; DEI. - West Region • 2 우; Dschang; [ $\left.5^{\circ} 27^{\prime} \mathrm{N}, 10^{\circ} 04^{\prime} \mathrm{E}\right]$; v. Rothkirch leg.; DEI.

CENTRAL AFRICAN REPUBLIC • 1 q; M. Boulard leg.; MNHN. - Ouham-Pendé • 1 q; Bozoum; [6¹9'02" N, 16 $6^{\circ} 22^{\prime} 42^{\prime \prime}$ E]; 11-20 Apr. 1914; G. Tessmann leg.; ZMB • 1 q; same collection data as for preceding but 1-10 May 1914; ZMB. 
DEMOCRATIC REPUBLIC OF THE CONGO • 1 q; “Kolo-Koilu-Madiata”; Sep. 1913; R. Verschueren leg.; MRAC • 1 क; "Kwango: Pangi"; 17 Feb. 1939; Mevr. Bequaert leg.; MRAC • 1 q; "Mulu sur Lua (Ubangi)"; 1931; Van Gils leg.; MRAC • 1 O; "Sankuru"; 1910; Abrassart leg.; MRAC • 1 ; Iringui [Kasai?]; Lindemans leg.; MRAC • 1 q; Katanga, Katampe; Jan. 1926; Ch. Seydel leg.; MRAC. - Bas-

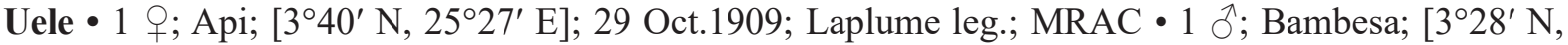
254' E]; 15 Sep. 1933; H.J. Brédo leg.; MRAC • 1 o; same collection data as for preceding but Feb.

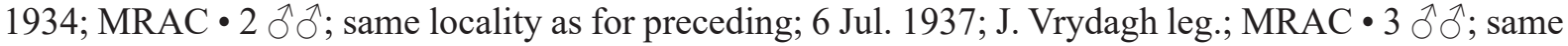
collection data as for preceding but 29 Aug. 1943; MRAC • 1 ; ; same collection data as for preceding but 1 Sep. 1943; MRAC • 1 ऊं; same locality as for preceding; 9 May 1938; P. Henrard leg.; MRAC • $1 \partial^{\top}$; same collection data as for preceding but May 1938; MRAC • 1 क ; Buta; [248' N, 2447' E]; 1932;

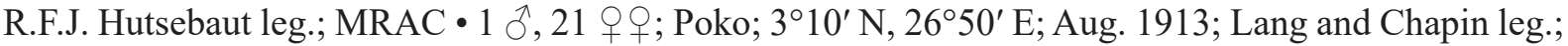

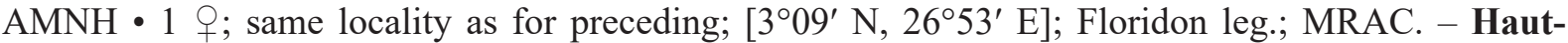

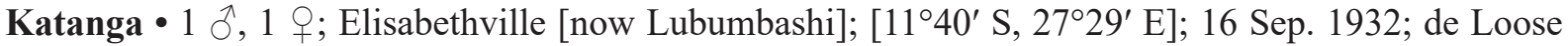

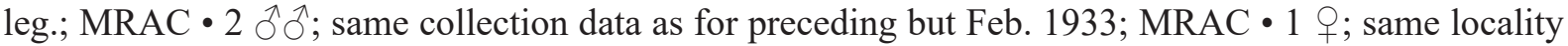
as for preceding; Jun. 1949; Ch. Seydel leg.; MRAC. - Haut-Lomami • 1 q; Kabongo; [7¹9' S,

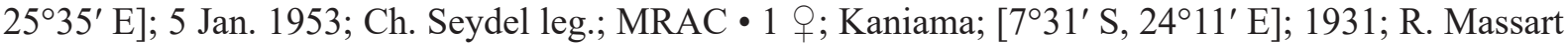
leg.; MRAC 1 O ; same collection data as for preceding but 1932; MRAC 11 क; Kanonga (675 m), P.N.U. [Upemba National Park]; [909' S, 2605' E]; 14-23 Feb. 1949; G.F. de Witte leg.; MRAC •

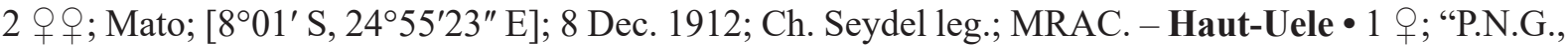
Miss. H. De Saeger" [Garamba National Park]; [ $4^{\circ} 00^{\prime}$ N, 291' E]; 10 Oct. 1951; H. De Saeger leg.; MRAC $\bullet 1$ q; same collection data as for preceding but Nov. 1951; MRAC $\bullet 1$ + ; Bafwabaca; $2^{\circ} 10^{\prime} \mathrm{N}$, $27^{\circ} 30^{\prime}$ E; Jan. 1910; Lang and Chapin leg.; AMNH 11 क; Faradje; $3^{\circ} 40^{\prime}$ N, $2^{\circ} 40^{\prime}$ E; Nov. 1912; Lang

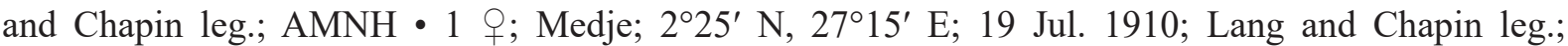
AMNH $-4 \hat{\partial} \hat{\partial}, 1$; ; same collection data as for preceding but Sep. 1910; AMNH - 3 q $O$; same collection data as for preceding but Jun. 1914; AMNH • 1 ; ; same collection data as for preceding but 9-15 Jul. 1910; AMNH • 1 O; Moto; [254'17" N, 29²7'22" E]; 1920; L. Burgeon leg.; MRAC. - Ituri

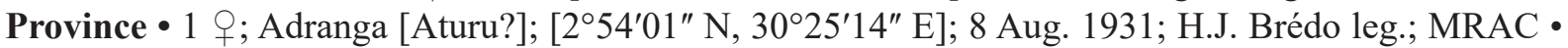

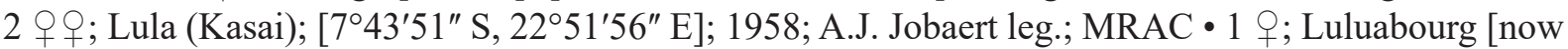
Kananga]; [553'46" S, 22²4'59" E]; Aug. 1956; M. Poll leg.; MRAC. - Kasaï-Oriental • 1 \&; Lukenge; [6 $6^{\circ} 14^{\prime} \mathrm{S}, 23^{\circ} 26^{\prime} \mathrm{E}$ ]; Fontainas leg.; MRAC. - Kinshasa - 1 \%; Leopoldville [now Kinshasa];

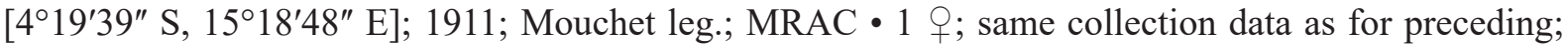
MRAC. - Kongo Central • 1 q; "Mayumbe: Buende-Suindi"; 15 May 1926; A. Collart leg.; MRAC •

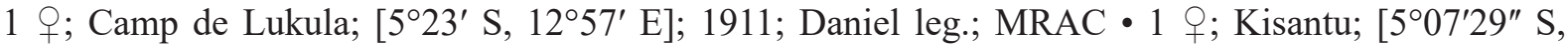

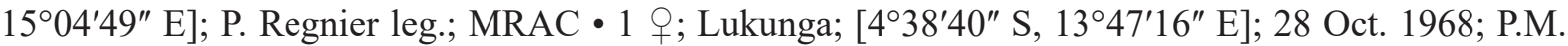

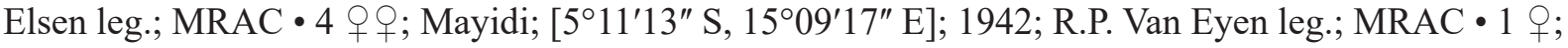

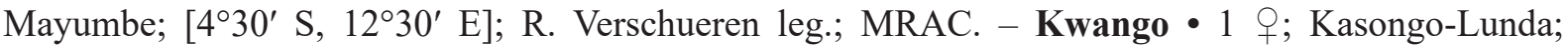
[6²8'42" S, 16²49'02" E]; Mar. 1971; V. Van Haelst leg.; MRAC. - Kwilu Province • 1 क; Gingungi;

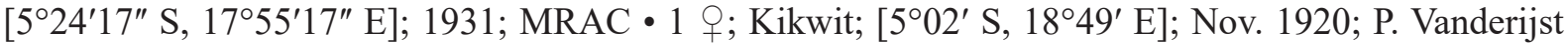

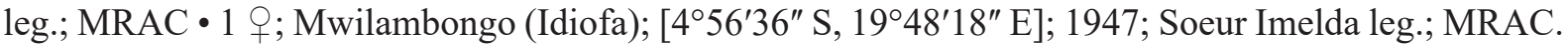
- Lomami • 1 O ; Kabinda; [6 $6^{\circ} 08^{\prime}$ S, 2429' E]; 1935; P. Henrard leg.; MRAC • 1 q; Kaniana; [6²6'23" S, 2350'58" E]; Jan. 1931; R. Massart leg.; MRAC. - Lualaba • 1 q; Bunkeya; [10²4' S,

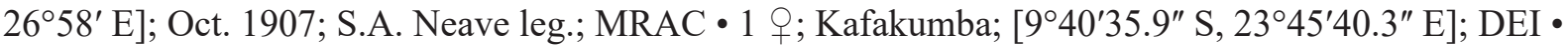

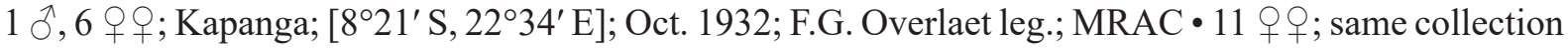
data as for preceding but Nov. 1932; MRAC $\bullet 1$ o; same collection data as for preceding but 3 Dec. 1932; MRAC • 2 q ; s same collection data as for preceding but 8 Dec. 1932; MRAC 2 q $O$; same collection data as for preceding but Jan. 1933; MRAC $\bullet 2$ 우; same collection data as for preceding but Feb. 1933; MRAC • 1 क; same collection data as for preceding but Oct. 1933; MRAC • 1 क; same collection data as for preceding but 1934; MRAC $\bullet 1$; same collection data as for preceding but Feb.

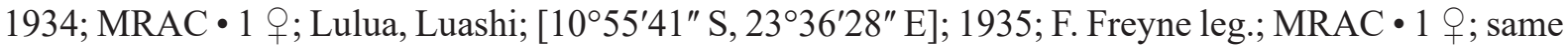
collection data as for preceding but 1936; MRAC 1 O ; Sandoa; [941' S, 2253' E]; 20 Mar. 1919; F.G. 


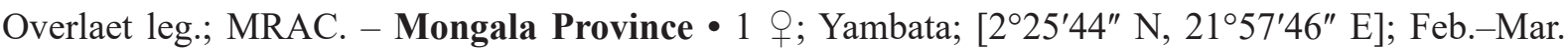

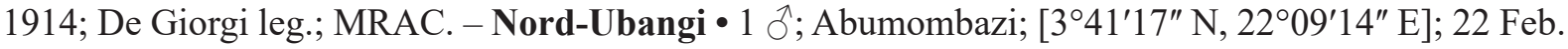
1932; H.J. Brédo leg.; MRAC • 1 क; same collection data as for preceding but 23 Feb. 1932; MRAC • 1 q; same collection data as for preceding but 18-26 Feb. 1932; MRAC • 1 o ; Bosobolo; [4¹1'23" N, 1952'59" E]; 8-11 Jan. 1932; H.J. Brédo leg.; MRAC • 1 q; Karawa; [3¹9'55" N, 20¹8'03" E]; 1936; Wallin leg.; MRAC. - North Kivu • 1 q; Beni; [0²9' N, 29²8' E]; Jul. 1911; Borgerhoff leg.; MRAC • 1 क; same locality as for preceding; Feb. 1931; L. Lebrun leg.; MRAC • 1 O; Mulo; [007'39" S, 29¹6'02" E]; Apr. 1950; R.P. Celis leg.; MRAC • 1 o ; Mutwanga, Semliki; [0²0'18" N,

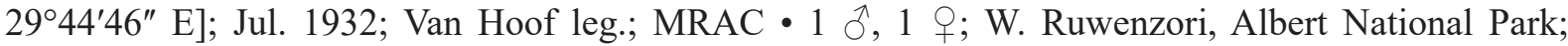

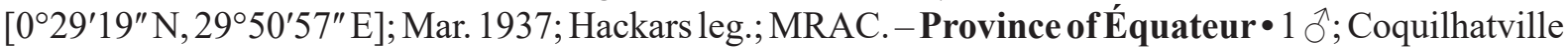
[now Mbandaka]; [003' N, 12 $\left.{ }^{\circ} 57^{\prime} \mathrm{E}\right]$; 1946; Ch. Scops leg.; MRAC • 1 + ; same locality as for preceding;

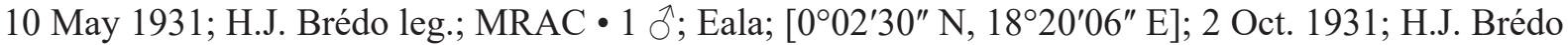
leg.; MRAC • 1 q; same collection data as for preceding but 3 Oct. 1931; MRAC • 1 \%; same collection data as for preceding but 7 Oct. 1931; MRAC 1 O ; same collection data as for preceding but 13 Oct.

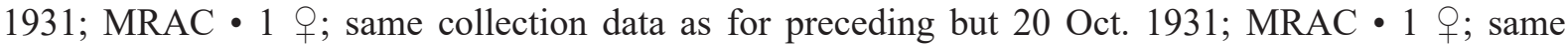
collection data as for preceding but Nov. 1931; MRAC $\bullet 1$ क; same collection data as for preceding but 5 Nov. 1931; MRAC • 2 q ; ; same collection data as for preceding but 14 Nov. 1931; MRAC • 1 q; same collection data as for preceding but 21 Nov. 1931; MRAC • 2 우; same collection data as for preceding but Mar. 1932; MRAC - 2 +9 ; same collection data as for preceding but Apr. 1932; MRAC • 1 \%; same collection data as for preceding but 22 Apr. 1932; MRAC $\bullet 1$ \% ; same collection data as for preceding but May 1932; MRAC $\bullet 2 \hat{\jmath}, 3$ 우; same locality as for preceding; Jun. 1932; A. Corbisier leg.; MRAC • 1 क ; same collection data as for preceding but 7 Jul. 1932; MRAC 1 ô, 1 क ; same locality as for preceding; May 1935; J. Ghesquière leg.; MRAC • 1 + ; same collection data as for preceding but Jul. 1936; MRAC • 1 \%; same collection data as for preceding but Aug. 1936; MRAC • 1 O; same collection data as for preceding but Dec. 1936; MRAC - 1 O ; Flandria; [0 $0^{\circ} 19^{\prime} 14^{\prime \prime} \mathrm{S}$, 1905'34" E]; 23 Aug. 1930; R.P.G. Hulstaert leg.; MRAC • 1 q; same collection data as for preceding but 1935; MRAC • 1 +; Ikengé; [ $0^{\circ} 06^{\prime} \mathrm{S}, 18^{\circ} 46^{\prime} \mathrm{E}$ ]; 1 Apr. 1914; R. Mayné leg.; MRAC・1 O; Lukolela; [1 $\left.{ }^{\circ} 03^{\prime} 37^{\prime \prime} \mathrm{S}, 17^{\circ} 10^{\prime} 55^{\prime \prime} \mathrm{E}\right]$; De Guide leg.; MRAC • 1 \%; Nouvelle Anvers [now Makanza]; $1^{\circ} 40^{\prime} \mathrm{N}$,

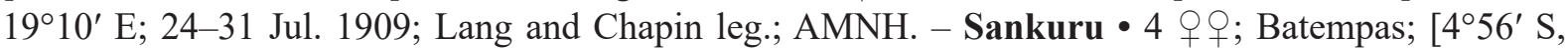

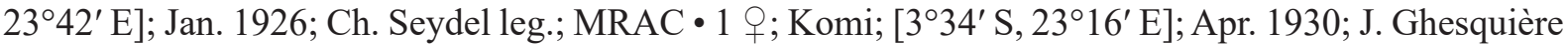
leg.; MRAC • 2 + $O$; same collection data as for preceding but May 1930; MRAC • 1 \%; same collection data as for preceding but Jul. 1930; MRAC • 1 क; Lonkala, forest; [4³7' S, 2313' E]; Apr. 1925; J. Ghesquière leg.; MRAC • 1 q; Niengele; Jan. 1926; Ch. Seydel leg.; MRAC. - Sud-Ubangi • 1 क; Binga; [2²2' N, 20²9' E]; Jan.-Mar. 1932; H.J. Brédo leg.; MRAC. - Tanganyika • 2 + $O$; "Lukuga r.

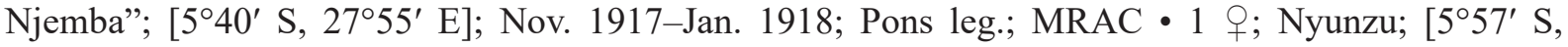
$28^{\circ} 01^{\prime}$ E]; Jan.-Feb. 1934; H. De Saeger leg.; MRAC • 1 q; Vallée Lukuga; [5³9'31" S, 26 54'53" E]; Nov. 1931; Schwetz leg.; MRAC. - Tshopo • 1 q; Avakubi; 1²0' N, 27³1' E; 4-8 Oct. 1909; Lang and Chapin leg.; AMNH • 1 q; same collection data as for preceding but 12 Oct. 1909; AMNH • 1 \%; Basoko; [1¹3’ N, 2336' E]; 1927; H.J. Brédo leg.; MRAC • 1 क; Basoko, Jaebeo [?]; Apr. 1949; P.L.G.

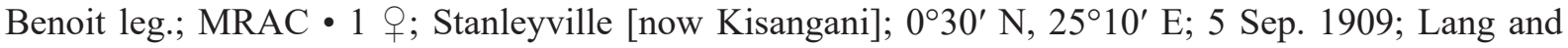
Chapin leg.; AMNH 1 1 ; same collection data as for preceding but Mar. 1915; AMNH • 2 o $\circ$; same collection data as for preceding but Apr. 1915; AMNH • 1 \% ; same collection data as for preceding but 4 Apr. 1915; AMNH • 1 q; same collection data as for preceding but 13 Apr. 1915; AMNH 1 \%; same collection data as for preceding but May 1915; AMNH - 1 क; Stanleyville [now Kisangani]; [0³0'55" N, 2511'27" E]; Apr. 1915; J. Bequaert leg.; MRAC • 1 q; same locality as for preceding; 20 Feb. 1928; A. Collart leg.; MRAC • 1 ; ; same collection data as for preceding but Sep. 1928; MRAC •

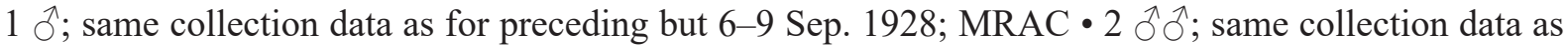
for preceding but 10-13 Sep. 1928; MRAC -1 o; same locality as for preceding; $10 \mathrm{Feb} .1932$; J. Vrydagh leg.; MRAC • 1 क; same collection data as for preceding but Jun. 1932; MRAC • 1 ○; same collection data as for preceding but 26 Jul. 1932; MRAC $\bullet 1$ \%; same locality as for preceding; Mar. 
1926; J. Ghesquière leg.; MRAC • 1 क ; Yangambi; [046’ N, 2427' E]; 17 Jun. 1948; P.L.G. Benoit leg.; MRAC • 1 \%; same locality as for preceding; May 1960; J. Decelle leg.; MRAC. - Tshuapa • 1 ; Bokuma; [006' S, $18^{\circ} 41^{\prime}$ E]; 1952; R.P. Lootens leg.; MRAC • 1 q; same collection data as for preceding but Mar. 1952; MRAC • 1 क ; same collection data as for preceding but Jun. 1952; MRAC 4 우; same collection data as for preceding but Jul. 1952; MRAC $\bullet 1 \widehat{\partial, 1}+$; same collection data as for preceding but 1953; MRAC • 1 क ; same collection data as for preceding but Mar. 1954; MRAC • 1 क; Bokungu; [041' S, 22¹9' E]; 1949; M. Dupuis leg.; MRAC • 2 우; Ikela; [1¹1' S, $23^{\circ} 16^{\prime}$ E]; 1955; R.P. Lootens

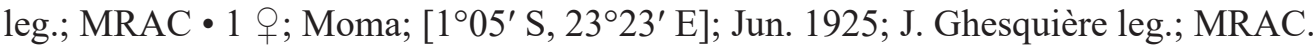

ERITREA 1 1 ; DEI.

GABON - Ogooué-Maritime Province - 1 q; $100 \mathrm{~km} \mathrm{~S}$ of Libreville, Bassin de la Bilagore; [0³0'24.3" S, 9³0'48" E]; 1938; M. Dumez leg.; MNHN.

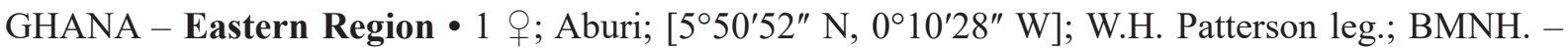

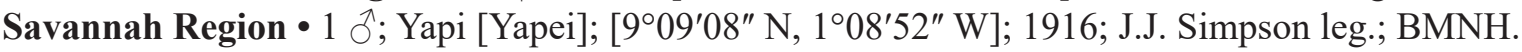

KENYA - Kilifi County • 1 क; Gedi Ruins, Malindi; $3^{\circ} 17^{\prime}$ S, 4002' E; 8-12 Nov. 1989; A.J. Weaving

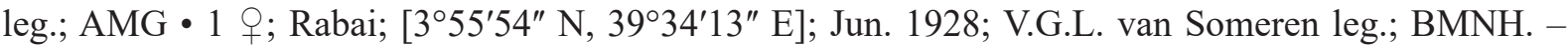

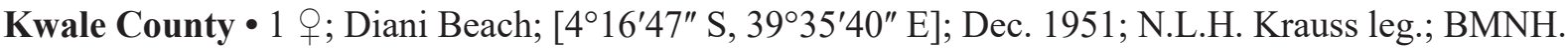
- Taita-Taveta County • 1 क; Voi River; [3²3'26" S, 38³4'37" E]; 14 Apr. 2007; M. Halada leg.; OÖLM.

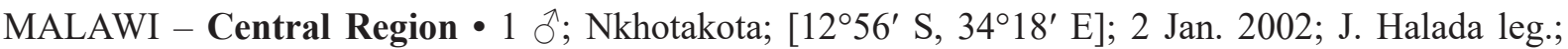

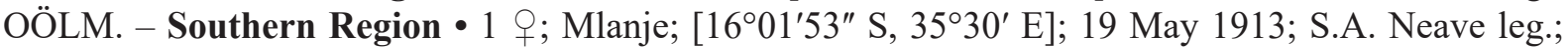
BMNH.

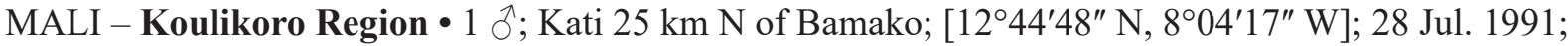
M. Schwarz leg.; OÖLM.

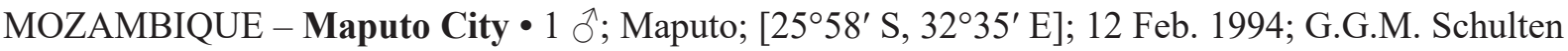
leg.; RMNH. - Sofala Province • 1 ${ }_{+}$; Beira; [1950’ S, 3451' E]; 1 Aug. 1903; P. Krantz leg.; TMP.

NIGERIA - Osun State • 1 क; Ile-Ife; [7²8'56" N, 433'37" E]; 25 Jan. 1974; J.T. Medler leg.; BMNH.

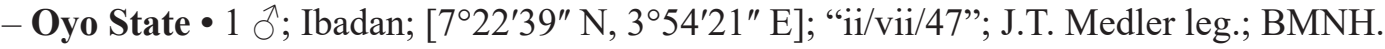

REPUBLIC OF CÔTE D'IVOIRE - Lagunes District - 1 क; Lamto Ecological Research Station; [6¹3'29.5" N, 502'4.8" W]; 15-30 Mar. 1994; C. Girard leg.; MNHN.

REPUBLIC OF THE CONGO - Brazzaville Department • 1 q; Brazzaville; [ $\left.4^{\circ} 16^{\prime} 04^{\prime \prime} \mathrm{S}, 15^{\circ} 17^{\prime} 31^{\prime \prime} \mathrm{E}\right]$; 21 Mar. 1966; MNHN. - Plateaux Department • 1 o ; Batéké, M'Po; [200'43.8" S, 16²18'30.7" E]; 10 14 Jan. 1977; S. Kellner-Pillaut leg.; MNHN. - Pool Department • 1 क; Kintelé; [409' S, 15²0'32" E]; 11 May 1978; G. Onore leg.; MNHN • 1 क; Mbé; [3¹8'13.68" S, 1553'51" E]; 27 Apr. 1973; C. Morin leg.; MNHN • 1 q; Voka; [440’16" S, 1440’25" E]; 11 Nov. 1976; G. Onore leg.; MNHN.

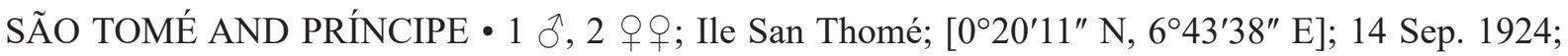
H. De Saeger leg.; MRAC • 1 \%; same collection data as for preceding but 19 Sep. 1924; MRAC •

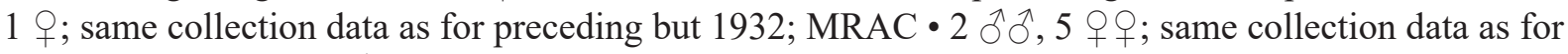
preceding; MRAC • 1 đं; same locality as for preceding; 1919-1921; H.J. Snell leg.; BMNH.

SIERRA LEONE - 1 , holotype or syntype of Sphex tuberculatus F. Smith, 1873; BMNH • 1 \%; Bumbumbo; 15 Aug. 1912; J.J. Simpson leg.; BMNH. - Western Area • 1 q; Freetown; [8 29'04" N, $13^{\circ} 14^{\prime} 04^{\prime \prime}$ W]; E.E. Austen leg.; BMNH. 
SOUTH AFRICA - Eastern Cape • 1 ○’; Sundays River; 25 Feb. 1901; O’Neil leg.; TMP. - KwaZuluNatal • 1 क ; Fanies Island; $28^{\circ} 06^{\prime} \mathrm{S}, 32^{\circ} 26^{\prime} \mathrm{E}$; AMG • 1 + ; Lake St Lucia, False Bay; $27^{\circ} 58^{\prime} \mathrm{S}, 32^{\circ} 23^{\prime} \mathrm{E}$; 27 Jan.-2 Feb. 1993; A.J. Weaving leg.; AMG • 1 क ; Lake St Lucia, Fanies Island; 2806' S, 32²6' E; 4-5 Mar. 1987; A.J. Weaving leg.; AMG. - Limpopo • 1 क; Lekgalameetse Nature Reserve; $24^{\circ} 11^{\prime}$ S, 30²1' E; 30 Oct.-3 Nov 2010; F. Koch leg.; THD-006-ZMB; GenBank CO1 gene: MW538551; GenBank EF-1 $\alpha$ gene: MW558239; GenBank LWR gene: MW582284; ZMB • 1 क; Sikororo [GaSekororo]; [24¹2'27.6" S, 30²4'33.3" E]; Jul. 1922; G.P.F. v. Dam leg.; TMP.

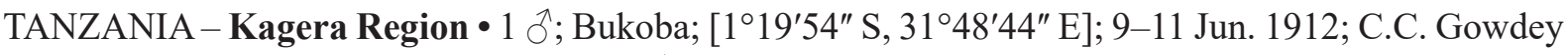
leg.; BMNH. - Kilimanjaro Region • 1 ơ; Kilimanjaro; [304'33" S, 37²1'12" E]; Y. Sjöstedt leg.; NRS. - Pwani Region • 1 क ; 15 km W of Kibiti; $7^{\circ} 47^{\prime}$ S, 38 $48^{\circ}$ ' E; 15 Jan. 2007; J. Halada leg.; OÖLM.

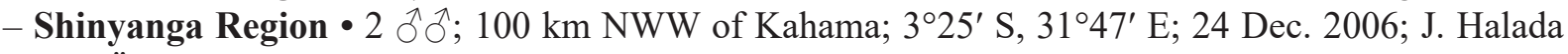
leg.; OÖLM.

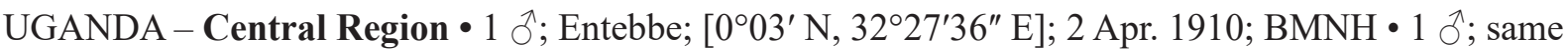
locality as for preceding; 15-22 Jun. 1912; C.C. Gowdey leg.; BMNH.

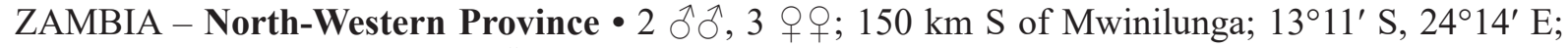
18 Oct. 2008; M. Halada leg.; OÖLM.

\section{Description}

Female

Size. $23.9-28.8 \mathrm{~mm}$.

CoLOR. Black except for the following, which are ferruginous: hindcoxa ventrally, hindtrochanter ventrally, hindfemur and proximal four-fifths of hindtibia. Cellular wing area hyaline, forewing with slightly fuscous spot beyond marginal cell.

VeSTITURE. Appressed and erect setae on clypeus and paraocular area golden, on collar and scutum brownish-golden. Appressed setae on propodeal enclosure silvery, erect setae silvery-golden. Erect propodeal setae crimped, oriented posteriorly. Clypeus without glabrous stripe. Scutellum densely and finely pubescent.

STRUCTURE. Free clypeal margin with broad, shovel-like process medially, not stepped above. Clypeus without indentation and carina. Scutellum convex. Metanotum raised, notably bituberculate. $2^{\text {nd }}$ recurrent vein joins markedly proximal from interstitium between submarginal cells II and III. Propodeal enclosure without any notable ridges. Foretarsomere I 2.1-2.6 $\times$ length of antepenultimate spine. Petiole length $3.4-3.6 \times$ its medial width.

\section{Male}

Size. $21.8-24.1 \mathrm{~mm}$.

CoLOR. Black except for the following, which are ferruginous: hindcoxa ventrally, hindtrochanter ventrally, hindfemur and proximal four-fifths of hindtibia. Cellular wing area hyaline, forewing with slightly fuscous spot beyond marginal cell.

Vestiture. Appressed and erect setae on clypeus and paraocular area golden, on collar and scutum brownish-golden. Appressed setae on propodeal enclosure silvery, erect setae silvery-golden. Erect propodeal setae crimped, oriented posteriorly. Clypeus without glabrous stripe. Scutellum densely and finely pubescent. 

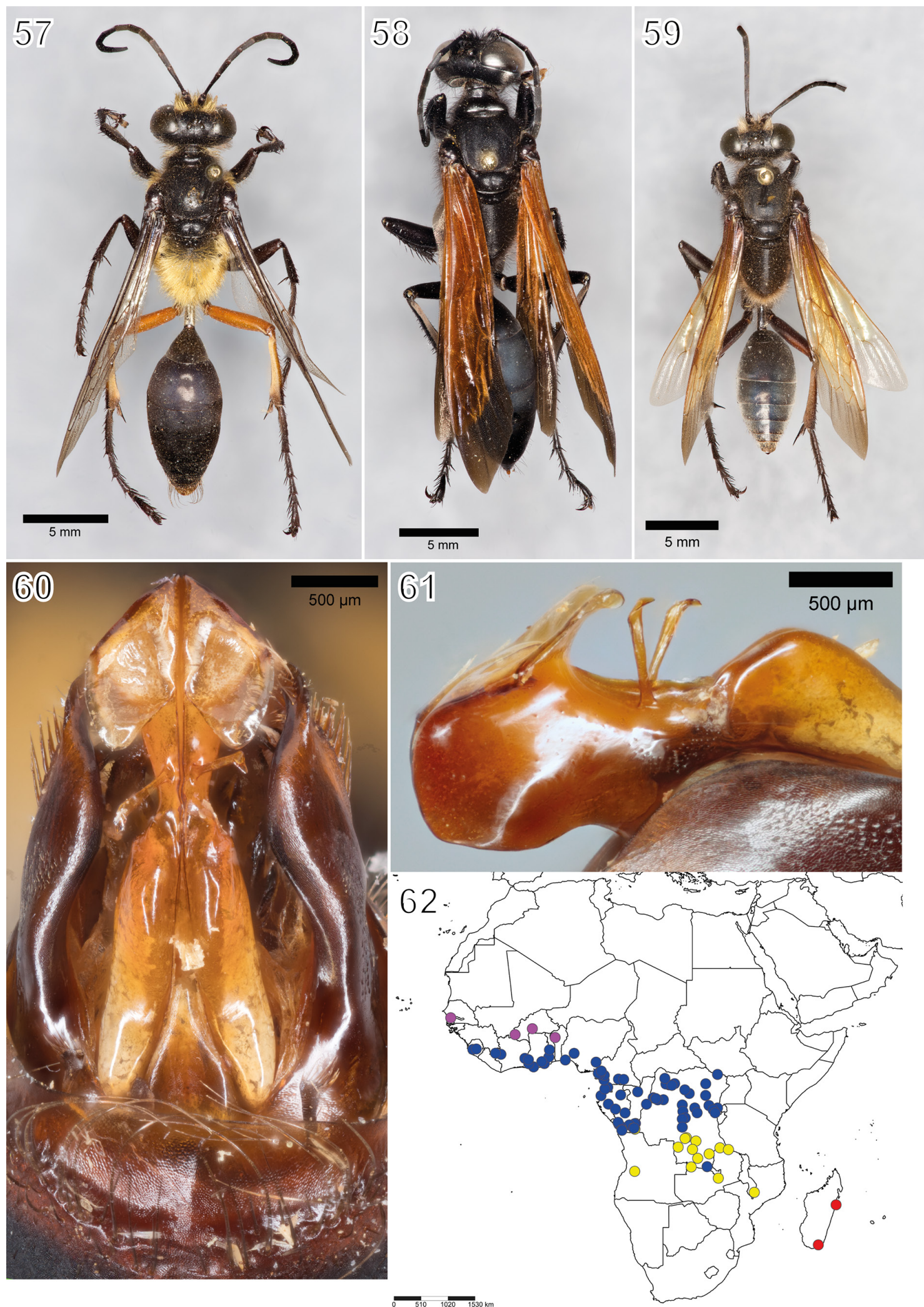

Figs 57-62. 57, 60-61. Sphex tomentosus Fabricius, 1787, đ. 58. S. torridus F. Smith, 1873, habitus of . 59. S. voeltzkowii Kohl, 1909, habitus of $\partial^{\lambda}$. 57. Habitus. 60. Dorsal view of genitalia. 61. Lateral view of penis valvae. 62. Geographic distribution of $S$. voeltzkowii (red), S. caeruleanus Drury, 1773 (blue), S. mweruensis (Arnold, 1947) (yellow) and S. hades sp. nov. (purple). 
Structure. Free clypeal margin simple. Scutellum convex. Metanotum raised, notably bituberculate. $2^{\text {nd }}$ recurrent vein joins markedly proximal from interstitium between submarginal cells II and III. Propodeal enclosure without any notable ridges. Metasomal tergum VII dorsally stepped, with curved long setae, its posterior margin convex. Posterior margin of metasomal sternum VII simple. Metasomal sternum VIII concave towards center, its posterior margin triangular. Penis valvae fused, dorsoventrally S-shaped, mediolaterally with long and thin process. Valviceps broadened, dorsally open and elongated towards anterior. Petiole length 3.4-3.6 $\times$ its medial width. Flagellomeres IV-VI with broad placoids covering their entire length.

\section{Variation}

In some males, the facial and propodeal setae are on the verge of being silvery.

\section{Distribution}

Western to eastern and southeastern Africa.

Sphex torridus F. Smith, 1873

Figs 56 (blue), 58

Sphex torridus F. Smith, 1873: 291, ․

\section{Differential diagnosis}

Within the argentatus group, this species (Fig. 58) can be recognized through its ferruginous or yellowbrownish wings and the erect setae on the face and propodeum being uniformly black. Females of S. voeltzkowii also have black erect facial and propodeal setae, but their wings are hyaline with a yellow tinge, and the coloration of the wing apices is different as well: forewing and hindwing margins are completely infuscate in $S$. torridus, whereas $S$. voeltzkowii only has a fuscous spot on the anterior part of the forewing apex, with the apical hindwing margin being hyaline. Additionally, males of S. voeltzkowii (Fig. 59) have a significant portion of the erect setae on the clypeus and paraocular area silvery or silvery-golden, while these are uniformly black in males of $S$. torridus. Sphex taschenbergi also has erect clypeal and propodeal setae that are black (Figs 52,54), but its wings are basally infuscate and otherwise hyaline.

\section{Material examined \\ Syntypes \\ MADAGASCAR • 2 + $;$ BMNH.}

\section{Other material}

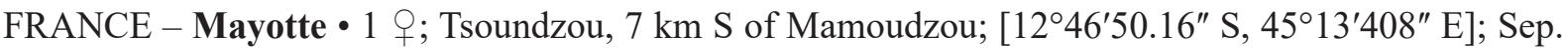
1986; R. and A. Viossat leg.; MNHN. - Mozambique Channel • $2{ }^{\lambda}{ }^{\lambda}, 1$ o ; Europa Island; [22 $22^{\prime} 06^{\prime \prime}$ S,

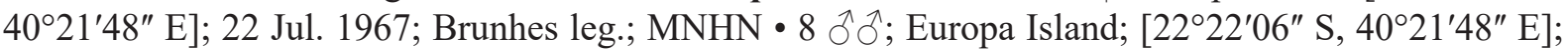
A. Voeltzkow leg.; ZMB.

MADAGASCAR • 1 đ, 2 우; ZMB. - Anosy Region • 1 क ; 9 km NE of Pt Dauphin, Forêt de Mandena;

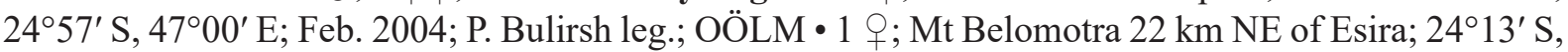
4653' E; Feb. 2004; P. Bulirsh leg.; OÖLM. - Atsimo-Andrefana Region - 1 q; Beza Mahafaly

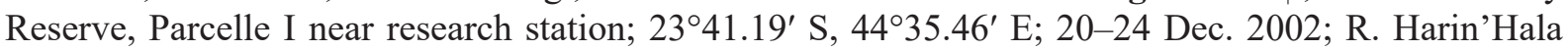
leg.; AMNH. - Atsinanana Region • $1 \delta^{\top}$; Toamasina; [18 $09^{\prime}$ S, 49 $25^{\prime}$ E]; DEI. - Boeny Region • 1 क; $40 \mathrm{~km}$ S of Marovoay; [16²8'17.2" S, 46³9'21.5" E]; 12 Mar. 1963; E.D. Cashatt leg.; USNM. -

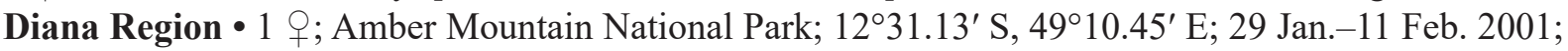
M. Irwin and R. Harin'Hala leg.; AMNH 1 1 ; same collection data as for preceding; MNHN 1 1 ; ; near 


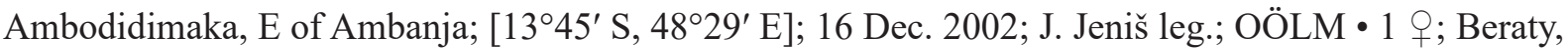

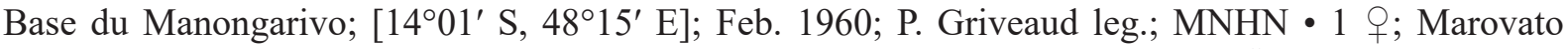

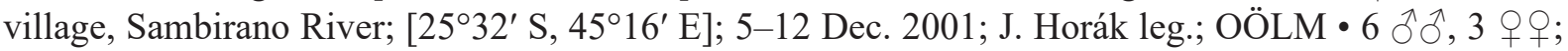

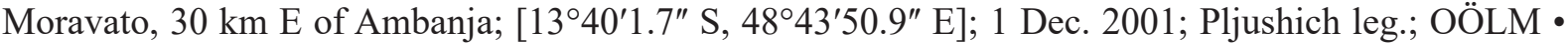
1 万ै; same collection data as for preceding; THD-017-OOLM; GenBank CO1 gene: MW538577; OÖLM.

- Ihorombe Region • $2 \partial^{\lambda} \partial^{\lambda}$; near Isalo National Park, in dry wash E of Interpretive Center; $22^{\circ} 37.60^{\prime} \mathrm{S}$, 45²1.49' E; 6-14 Dec. 2002; M. Irwin and R. Harin'Hala leg.; AMNH. - Melaky Region • 1 q; Antsingy Nature Reserve, Antsalova; [18³8' S, 4854' E]; 9 Jan. 1975; A. Peyrieras leg.; MNHN. - Sava Region •

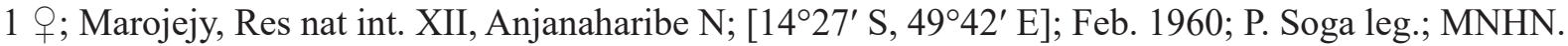

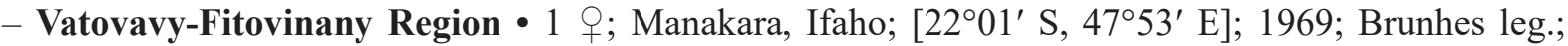

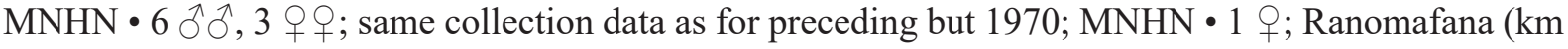
402); [21ำ $15^{\prime}$ S, $47^{\circ} 27^{\prime}$ E]; alt. 650 m; 26 Mar.-1 Apr. 1955; H. de Toulgoët leg.; MNHN.

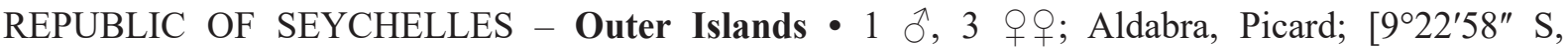

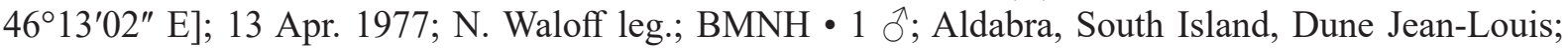
[9²6'43" S, 46²3'21.5" E]; 13-20 Mar. 1968; B. Cogan and A. Hutson leg.; BMNH • 1 q; Aldabra,

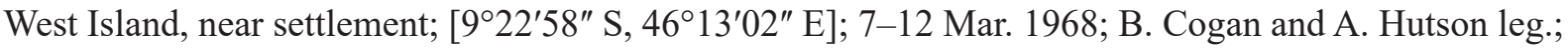

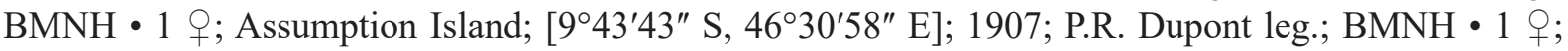
Astove Island; [1006' S, 4745' E]; 1907; P.R. Dupont leg.; BMNH.

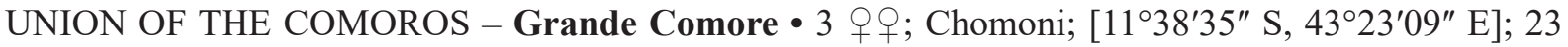

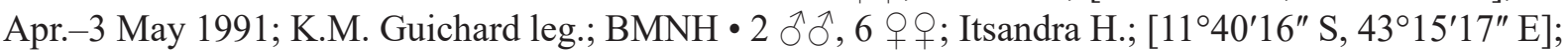
23 Apr.-3 May 1991; K.M. Guichard leg.; BMNH.

\section{Description}

Female

SIZE. $18.5-28.3 \mathrm{~mm}$.

CoLOR. Black except for basal half of mandible, which is ferruginous. Cellular wing area ferruginousbrown, apical margin fuscous.

VeSTITURE. Appressed setae on clypeus, paraocular area and collar silvery, on scutum and propodeal enclosure black. Erect setae on clypeus, paraocular area, collar and scutum black, on propodeal enclosure brown or black. Erect propodeal setae oriented anteriorly. Clypeus without glabrous spot. Scutellum densely and finely pubescent.

StRUCTURE. Free clypeal margin slightly notched medially, not stepped above. Clypeus without indentation or carina. Scutellum convex. Metanotum raised, notably bituberculate. Propodeal enclosure finely transversely carinate. $2^{\text {nd }}$ recurrent vein joins slightly proximal from interstitium between submarginal cells II and III. Propodeal enclosure without any notable ridges. Foretarsomere I 2.1-2.2× length of antepenultimate spine. Petiole length $1.7-2.0 \times$ its medial width.

Male

Size. 22.0-25.5 mm.

CoLOR. Black, except for ferruginous stripe in center of mandible. Cellular wing area yellowish-brown, base of fore- and hindwing infuscate. Forewing with fuscous spot beyond marginal cell.

VeSTITURE. Appressed setae on clypeus, paraocular area and collar silvery, on scutum and propodeal enclosure black. Erect setae on clypeus, paraocular area, collar and scutum black, on propodeal enclosure 
brown or black. Erect propodeal setae oriented anteriorly. Clypeus without glabrous spot. Scutellum densely and finely pubescent.

StruCture. Free clypeal margin simple. Scutellum convex. Metanotum raised, notably bituberculate. Propodeal enclosure finely transversely carinate. $2^{\text {nd }}$ recurrent vein joins slightly proximal from interstitium between submarginal cells II and III. Propodeal enclosure without any notable ridges. Posterior margin of metasomal tergum VII convex. Posterior margin of metasomal sternum VII simple, of metasomal sternum VIII triangular. Penis valvae without conspicuous modifications. Petiole length 2.7-3.0 $\times$ its medial width. Flagellomeres V + VI with broad placoids covering their entire length.

\section{Variation}

Unknown.

\section{Distribution}

Comoros, Europa Island, Madagascar, Seychelles.

Sphex voeltzkowii Kohl, 1909

Figs 59, 62 (red)

Sphex umbrosus var. voeltzkowii Kohl, 1909: 370, §, ㅇ. .

\section{Differential diagnosis}

Within the argentatus group; both sexes of this species can be recognized through the combination of the erect setae on the clypeus and the propodeum being at least partially black and the wings being slightly yellowish and basally not infuscate (Fig. 59). Similarly, S. taschenbergi and S. torridus have the erect clypeal and propodeal setae uniformly black, but the wings of $S$. taschenbergi are fuscous near the base and otherwise hyaline (Fig. 52), whereas the cellular wing area of S. torridus is markedly ferruginousbrown (Fig. 58). Females of $S$. voeltzkowii are furthermore unique within the argentatus group regarding their more or less distinct elevation near the dorsal clypeal margin.

\section{Material examined}

Lectotype (designated here)

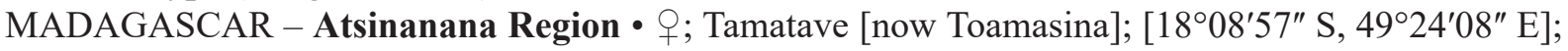
Nov. 1904; ZMB.

Paralectotype

MADAGASCAR - Atsinanana Region • $1 \partial^{\lambda}$; same collection data as for lectotype; ZMB.

\section{Other material}

AFRICA • 1 क ; BMNH.

MADAGASCAR - Anosy Region • 2 우우 ; Behara; [245ㄱ' S, 46²3' E]; Jan. 1938; A. Seyrig leg.; $\mathrm{MNHN} \bullet 1$ đ, 1 q; same collection data as for preceding but Nov. 1938; MNHN.

\section{Description}

Female

Size. 25.7-26.8 mm.

COLOR. Black except for the following, which are ferruginous: basal half of mandible and hindfemur posterodorsally. Cellular wing area yellowish. Forewing with fuscous spot beyond marginal cell. 
VeSTITURE. Appressed setae on clypeus, paraocular area and collar silvery, on scutum and propodeal enclosure black. Erect setae on clypeus, paraocular area, collar, scutum and propodeal enclosure black. Erect propodeal setae crimped, oriented perpendicularly and slightly curved towards anterior. Clypeus without glabrous spot. Scutellum densely and finely pubescent.

StRUCTURE. Free clypeal margin with three notches medially, not stepped. Clypeus without indentation, with elevation near dorsal margin. Scutellum convex. Metanotum raised, notably bituberculate. $2^{\text {nd }}$ recurrent vein joins slightly proximal from interstitium between submarginal cells II and III. Propodeal enclosure without any notable ridges. Foretarsomere I 2.6-2.8 $\times$ length of antepenultimate spine. Petiole length $1.6-2.0 \times$ its medial width.

\section{Male}

Size. 22.1-23.5 mm.

CoLOR. Black except for ferruginous stripe in center of mandible. Cellular wing area yellowish. Forewing with fuscous spot beyond marginal cell.

VESTITURE. Appressed setae on clypeus, paraocular area and collar silvery, on scutum silvery-golden, on propodeal enclosure brownish-golden. Erect setae on clypeus, scutum and propodeal enclosure black and brownish-golden, on paraocular area and collar black, brownish-golden and silvery. Erect propodeal setae crimped, oriented perpendicularly and slightly curved towards anterior. Clypeus with glabrous spot in lower center. Scutellum densely and finely pubescent.

STRUCTURE. Free clypeal margin simple. Scutellum convex. Metanotum raised, notably bituberculate. $2^{\text {nd }}$ recurrent vein nearly joins interstitium between submarginal cells II and III. Propodeal enclosure without any notable ridges. Posterior margin of metasomal tergum VII convex. Posterior margin of metasomal sternum VII simple, of metasomal sternum VIII triangular. Penis valvae without conspicuous modifications. Petiole length $2.0-2.5 \times$ its medial width. Flagellomere II with or without broad placoid covering its proximal two-thirds, flagellomeres $\mathrm{V}+\mathrm{VI}$ with broad placoids covering their proximal four-fifths.

\section{Variation}

Unknown.

\section{Distribution}

Madagascar.

\section{Species of the umtalicus group}

The umtalicus group contains the following six species: Sphex caeruleanus, S. hades sp. nov., S. haemorrhoidalis, S. mweruensis, S. umtalicus and $S$. victoria sp. nov.

The members of this group can be recognized mainly through a combination of the following two characters: The erect propodeal setae are oriented more or less perpendicularly or slightly curved toward anterior (Fig. 3-4), and the scutellum (which is often markedly impressed) is always covered with coarse and dense appressed setae, in contrast to the fine setae that are characteristic for the meridionalis group, of which the members sport similarly oriented erect propodeal setae. Species of the malagassus group are somewhat similar in that their erect propodeal setae are perpendicular, but without curvature, and can be distinguished through their appressed silvery-golden setae on the scutum and metanotum. In addition, they only occur on Madagascar and the surrounding islands, respectively. 
Males in the umtalicus group are additionally characterized by having a conspicuous process medially at the free clypeal margin. The only other species with a comparable structure are $S$. cinerascens and S. abyssinicus.

Species delimitation within the group is very difficult, since intraspecific variation is high and hybrid forms seem to be quite common, even though most of the taxa are apparently genetically distinct. Colorrelated characters are of greater diagnostic importance within this group, and the geographical origin of the specimen should always be taken into consideration to help with identification.

\section{Sphex caeruleanus Drury, 1773}

Figs 62 (blue), 63, 67

Sphex caeruleanus Drury, 1773: 74, pl. 39 fig. 4, $q$ (holotype: + , Africa, Bite of Benin, destroyed?).

Sphex pulchripennis Mocsáry, 1883: 35, $q$ (holotype or syntype: $q$, Ghana, Ashanti Region, no specific locality, TMB, not examined). Synonymized with Sphex caeruleanus by Menke in Bohart \& Menke 1976: 114.

\section{Differential diagnosis}

This species is characterized by its large size, predominantly greenish wing iridescence and uniformly black erect mesosomal setae (Fig. 63). All other members of the group have a body length of less than $26 \mathrm{~mm}$, and most of the time their wing iridescence is mainly violet or cyan-violet. Furthermore, female specimens are identifiable through their largely black clypeus, which is often ferruginous medially near the free margin and covered with appressed silvery setae (Fig. 67). Sphex victoria sp. nov. (Fig. 35) and S. mweruensis (Fig. 68), both of which sport silvery appressed clypeal setae in the female sex, have the clypeus almost or entirely ferruginous.

Males of $S$. caeruleanus differ from other group members in having the width of the process at the free clypeal margin $0.15-0.2 \times$ the distance between the inner orbits at the clypeal center and concavely emarginate apically. Sphex mweruensis is similar in the shape of its free clypeal margin and process, and can have the wings with a notable greenish iridescence as well, but is distinguishable through its silvery erect propodeal setae and because the width of the clypeal process is only around $0.1 \times$ the distance between the inner orbits.

\section{Material examined}

AFRICA 1 1 ; DEI $\bullet 1$ \% IRSN $・ 1$ \% ZMB.

CAMEROON ・ 1 q; 6 Aug. 1923; AMNH • 5 oㅇ; L. Conradt leg.; DEI ・ 1 क; P. Preuss leg.; ZMB • 4 우; Y. Sjöstedt leg.; NRS • 1 q; "Esudan-Mamfe"; Guillemain leg.; ZMB. - East Region • 2 우; Batouri District; $3.45^{\circ}$ N, $13.45^{\circ}$ E; 1 May-6 Jun. 1935; F.G. Merfield leg.; BMNH • 3 q $ᄋ$; D'Ja Posten; $3.15^{\circ} \mathrm{N}, 13.30^{\circ}$ E; 15 May-1 Jul. 1936; F.G. Merfield leg.; BMNH. - Littoral Region • 3 q $ᄋ$; Nlobe-Ndonga between Edéa and Douala; [ $3^{\circ} 50^{\prime} 41.3^{\prime \prime}$ N, $10^{\circ} 00^{\prime} 07.8^{\prime \prime}$ E]; Schäfer leg.; ZMB. - South Region - 1 q; Ekok, 24 mi. E of Tekmo; [ $3^{\circ} 12^{\prime} 19^{\prime \prime}$ N, 12 $25^{\prime} 25^{\prime \prime}$ E]; 11-12 Oct. 1966; E.S. Ross and K. Lorenzen leg.; CAS • 1 क; Longji; [304'40.7" N, 958'29.1" E]; H. Paschen leg.; ZMB. - Southwest Region • 1 đ̃; "Johann-Albrechts-Höhe" [Government Station Johann Albrecht Mountain]; Mar. 1896; L. Conradt leg.; ZMB • 2 우; Bibundi; [4¹3'09" N, 859'15" E]; 1-15 Feb. 1905; G. Tessmann leg.; ZMB • 1 क ; same collection data as for preceding but 15-30 Apr. 1905; ZMB • 1 \%; Mukonye Farm;

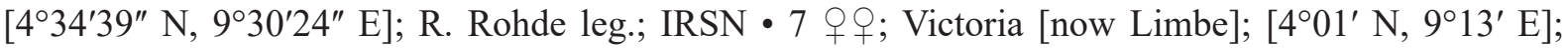
P. Preuss leg.; ZMB. 
DEMOCRATIC REPUBLIC OF THE CONGO • 1 \%; Goosens leg.; MRAC • 1 q; "Kasai”; L. Achten leg.; MRAC • 1 क ; same collection data as for preceding; MRAC • 1 क; "Ubangi: Tongu"; 4 Feb. 1932; H.J. Brédo leg.; MRAC • 1 q ; Bokote "Bolete Wa Bondele"; 24 Jan. 1926; R.P.G. Hulstaert leg.; MRAC• 1 q; Eala-Bokatora-Bikolo; Sep.-Oct. 1930; P. Staner leg.; MRAC • 1 q; Kasai, Lotima; 28 Jan. 1906; Waelbroeck leg.; MRAC. - Haut-Katanga • 1 + ; Elisabethville [now Lubumbashi]; [11 $40^{\prime} \mathrm{S}, 2^{\circ} 29^{\prime} \mathrm{E}$;;

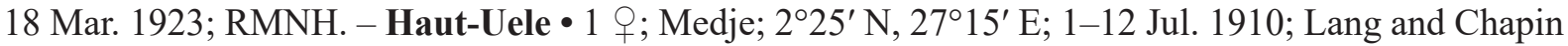
leg.; AMNH. - Kinshasa • 1 क; Leopoldville [now Kinshasa]; [4¹9'39" S, $15^{\circ} 18^{\prime} 48^{\prime \prime}$ E]; L. Achten leg.;

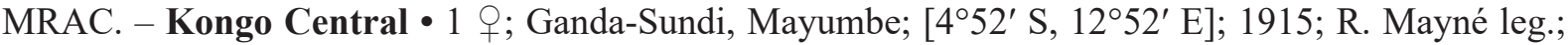

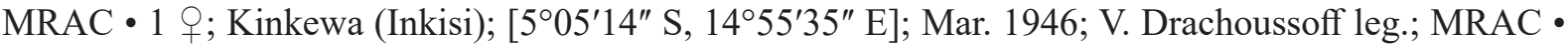

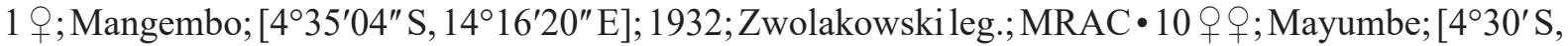
$12^{\circ} 30^{\prime}$ E]; 1917; R. Mayné leg.; MRAC • 1 क; Sanda near Kisantu; [ $\left.5^{\circ} 07^{\prime} 32.51^{\prime \prime} \mathrm{S}, 15^{\circ} 04^{\prime} 22.47^{\prime \prime} \mathrm{E}\right]$;

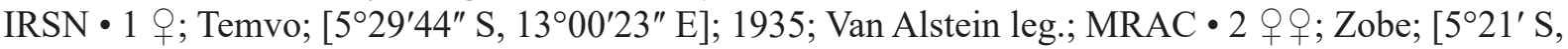
1301' E]; Jan. 1916; R. Mayné leg.; MRAC. - Maniema - 1 q; Lokandu, Ile Biawa; [2 $31^{\prime}$ S,

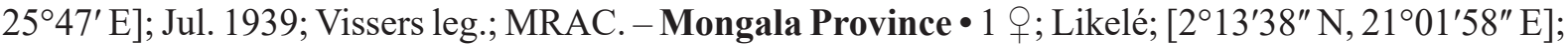
12 Jun. 1936; J. Ghesquière leg.; MRAC • 2 क 9 ; Yambata; [2²5'44" N, $21^{\circ} 57^{\prime} 46^{\prime \prime}$ E]; Feb.-Mar. 1914;

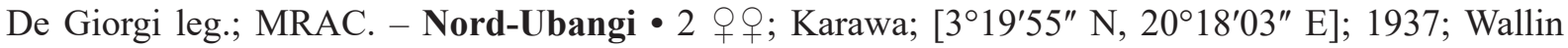

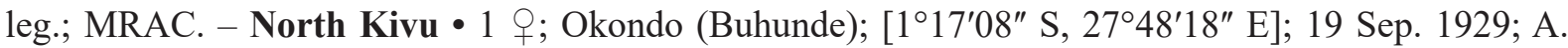
Collart leg.; MRAC • 1 \%; Oso River; [ $\left.4^{\circ} 00^{\prime} \mathrm{N}, 2^{\circ} 15^{\prime} \mathrm{E}\right]$; 18 Jan. 1915; J. Bequaert leg.; MRAC. Province of Équateur • 1 q; Bamania; [ $\left.0^{\circ} 01^{\prime} \mathrm{N}, 18^{\circ} 19^{\prime} \mathrm{E}\right]$; 1934; R.Fr. Longinus leg.; MRAC • 5 o $o$; Eala; [002'30" N, 18²0'06" E]; 1932; A. Corbisier leg.; MRAC • 2 우; same collection data as for preceding but Mar. 1932; MRAC • 1 \%; same collection data as for preceding but Jun. 1932; MRAC • 1 क; same collection data as for preceding but Jul. 1932; MRAC • 3 + 9 ; same collection data as for preceding but Apr. 1933; MRAC • 1 O ; same collection data as for preceding but Mar. 1935; MRAC • 1 q; same locality as for preceding; Oct. 1929; H.J. Brédo leg.; MRAC • 1 ${ }^{\text {; }}$ same collection data as for preceding but 5 Nov. 1931; MRAC • 1 q; same collection data as for preceding but Apr. 1932; MRAC • 1 q; same collection data as for preceding but 4 Apr. 1932; MRAC $\bullet 1$; ; same collection data as for preceding but May 1932; MRAC 4 $Q$ Q ; same locality as for preceding; May 1935; J. Ghesquière leg.; MRAC • 1 क ; same collection data as for preceding but Jan. 1936; MRAC • 1 \%; same collection data as for preceding but Apr. 1936; MRAC • 1 क ; same collection data as for preceding but Jul. 1936; MRAC • 1 o; same collection data as for preceding but Aug. 1936; MRAC 1 1 ; same collection data as for preceding but Oct. 1936; MRAC • 1 O ; same collection data as for preceding but Nov. 1936; MRAC •

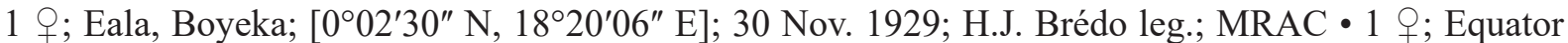
station [Bolenge]; [ $\left.0^{\circ} 00^{\prime} 0.36^{\prime \prime} \mathrm{N}, 18^{\circ} 13^{\prime} 0.12^{\prime \prime} \mathrm{E}\right]$; A. van Gèle leg.; IRSN • 1 क; Flandria; [0 $0^{\circ} 19^{\prime} 14^{\prime \prime} \mathrm{S}$, 1905'34" E]; Jan.-Feb. 1948; R.P.G. Hulstaert leg.; MRAC • 1 q; Ikengé; [006' S, 1846' E]; 1 Apr.

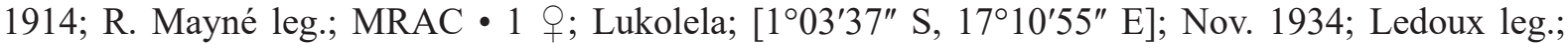
MRAC • 2 우; same locality as for preceding; Oct.-Dec. 1941; Breulheid leg.; MRAC. - Sankuru •

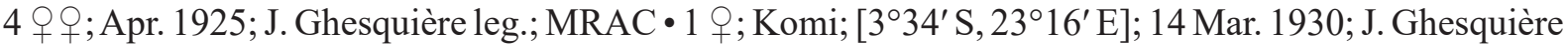
leg.; MRAC • 1 ㅇ; same collection data as for preceding but 26 Mar. 1930; MRAC • 1 \% ; same collection data as for preceding but Apr. 1930; MRAC 1 1 ; same collection data as for preceding but Apr.-May 1930; MRAC 11 우; same collection data as for preceding but May 1930; MRAC 7 우; same

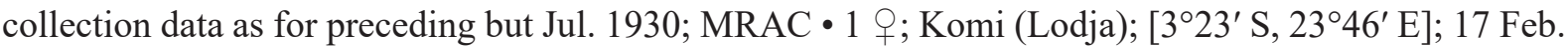
1930; J. Ghesquière leg.; MRAC • 1 क ; same collection data as for preceding but Mar. 1930; MRAC •

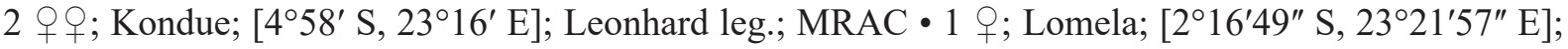

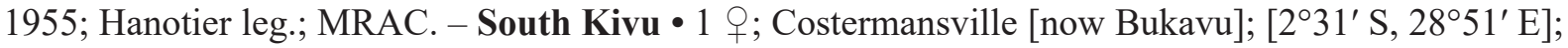
1939; Hautmann leg.; MRAC • 7 우; Kavumu à Kabunga km82 (Mingazi); [201' S, 28³1' E]; 1951; H. Bomans leg.; MRAC. - Sud-Ubangi • 1 P; Binga; [2²2' N, 20²9' E]; Jan.-Mar. 1932; H.J. Brédo

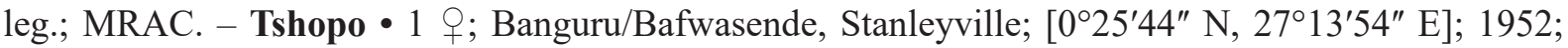
Abbeloos leg.; MRAC • 1 क; Basoko, Yamabuki; [1 ${ }^{\circ} 23^{\prime} \mathrm{N}, 2^{\circ}{ }^{\circ} 42^{\prime}$ E]; 16 Mar. 1948; P.L.G. Benoit leg.; MRAC • 2 우; Lukungu; [ $\left.1^{\circ} 42^{\prime} \mathrm{S}, 2^{\circ} 23^{\prime} \mathrm{E}\right]$; Ch. Haas leg.; IRSN • 2 우; Yangambi; [0 $0^{\circ} 46^{\prime} \mathrm{N}$,

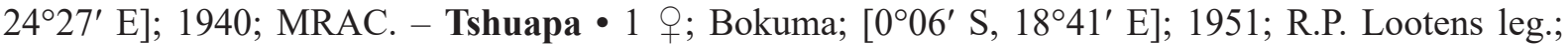


MRAC • 1 क; same collection data as for preceding but Dec. 1951; MRAC $\bullet 1$ \% ; same collection data as for preceding but 1952; MRAC $\bullet 16$ 우; same collection data as for preceding but Jul. 1952; MRAC • 3 우; same collection data as for preceding but 1953; MRAC - 1 영 Ikela; [1 $\left.{ }^{\circ} 11^{\prime} \mathrm{S}, 23^{\circ} 16^{\prime} \mathrm{E}\right]$; Oct. 1959; N. Leleup leg.; MRAC • 1 क; Terr. Boende; Yanga; [0¹8’ S, 2003’ E]; 5 Nov. 1949; E. Pauquet leg.; MRAC.

EQUATORIAL GUINEA - Centro Sur • 1 q; Monte Alén, Benito River; [1 ${ }^{\circ} 31^{\prime} 48^{\prime \prime}$ N, $10^{\circ} 06^{\prime} 36^{\prime \prime}$ E]; 16-31 Dec. 1906; G. Tessmann leg.; ZMB • 1 \%; same collection data as for preceding but 1-14 Jan. 1907; ZMB • 2 우; Uelleburg; [149’ N, 10³6' E]; Jun.-Aug. 1908; G. Tessmann leg.; ZMB.

GABON - Estuaire Province • 1 क; Libreville and environs; [0²3'24.36" N, 9²7'15.84" E]; MNHN. - Ngounié Province • 2 + $ᄋ$; “Chûtes de Samlia; Rivière N'Gami” [Ngounié River, chute de Samba]; [1 $\left.{ }^{\circ} 02^{\prime} 59.9^{\prime \prime} \mathrm{S}, 10^{\circ} 42^{\prime} \mathrm{E}\right]$; A. Mocquerys leg.; IRSN. - Ogooué-Ivindo • 1 क; Ipassa Research Station; [0³0'47" N, 1248'10" E]; 20 Apr.-16 May 1974; M. Donskoff and J. Le Breton leg.; MNHN.

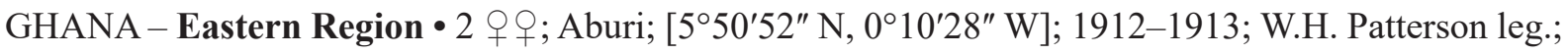
BMNH • 2 우; Kili District, Mt Atewa; [609'48" N, 0³6'41" W]; 15 Mar. 1969; O.W. Richards leg.; BMNH. - Western Region • 1 ; 7 miles N of Sefwi Asempanaye; 630' N, 253' W; 17 May. 1972;

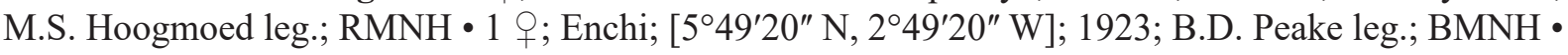

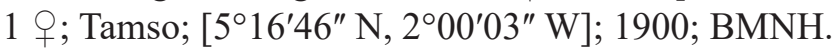

NIGER・ 1 \%; 1897?; Crosse leg.; BMNH.

NIGERIA • 1 đ’; 1919; BMNH. - Cross River State • 1 o ; Ikom; [605’ N, 8³7’ E]; 4 Apr. 1975; J.T. Medler leg.; BMNH. - Lagos State • 1 क; Lagos; [6²7'14" N, 3²3'40" E]; Nov. 1944; K.M. Guichard leg.; BMNH. - Osun State • 1 क; Erin-Odo; [7³5’ N, 453' E]; 9 Dec. 1973; J.T. Medler leg.; BMNH.

REPUBLIC OF CÔTE D'IVOIRE - Indénié-Djuablin Region • 1 ơं; Abengourou; [ $6^{\circ} 44^{\prime} \mathrm{N}, 3^{\circ} 29^{\prime} \mathrm{W}$ ];

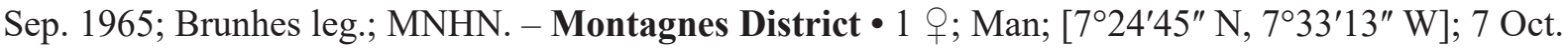
1961; J. Decelle leg.; MRAC • 1 क; Mt Nimba; [7³6’09" N, 8²8'5.6" W]; 24 Jun. 1958; P. Etuys leg.; RMNH.

REPUBLIC OF THE CONGO • 2 q ; " "Les sanas = yirard"; 27 Jan. 1977; J.-P. Grillot and C. Morin

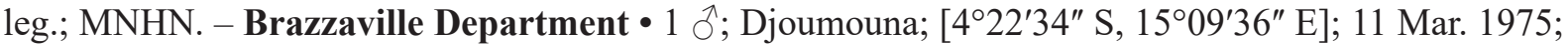

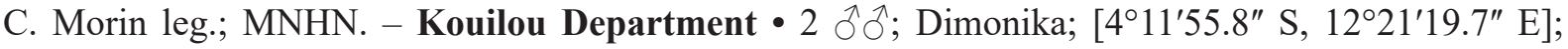
Jan. 1964; A. Descarpentries and A. Villiers leg.; MNHN • 1 q; same locality as for preceding; 20 Jan. 1977; J.-P. Grillot and C. Morin leg.; MNHN • 4 우; same collection data as for preceding but 18 May 1977; MNHN • 1 o, 1 q; same locality as for preceding; 7 Feb. 1978; C. Morin leg.; MNHN • 1 q; same collection data as for preceding but $8 \mathrm{Feb} .1978$; MNHN $\bullet 2$ o $o+$; same collection data as for preceding but 20 Feb. 1978; MNHN • 1 \%; same locality as for preceding; 23 Feb. 1978; J.-J. Menier

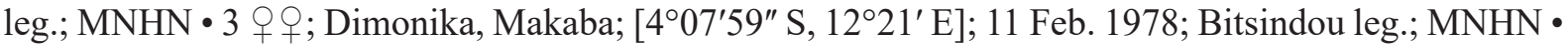
1 q; Dimonika, Piste de Kolela; [4¹1'55.8" S, 12²1'19.7" E]; 8 Feb. 1978; J.-J. Menier leg.; MNHN •

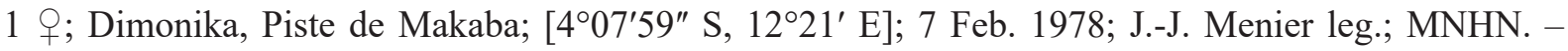

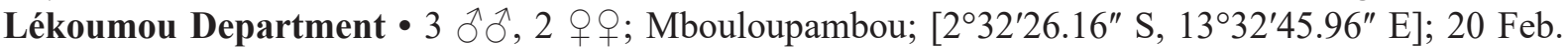
1980; C. Morin leg.; MNHN. - Sangha Department • 3 우; Ngongo; [ $1^{\circ} 02^{\prime} 58^{\prime \prime}$ N, $15^{\circ} 41^{\prime} 16^{\prime \prime}$ E]; 25 Feb. 1970; J.-P. Grillot leg.; MNHN.

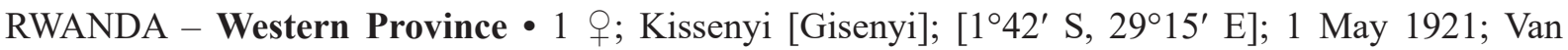
Saceghem leg.; MRAC. 
SIERRA LEONE - Eastern Province • 1 đ̧; Tungea; 12 Sep. 1912; J.J. Simpson leg.; BMNH. Southern Province • 1 \%; Gbangbama; [ $8^{\circ} 14^{\prime} 50^{\prime \prime}$ N, 12 $2^{\circ} 19^{\prime} 47^{\prime \prime}$ W]; 10 Oct. 1912; J.J. Simpson leg.;

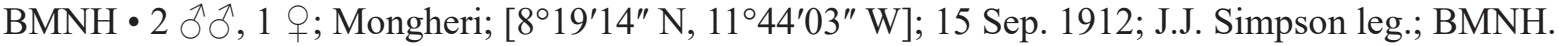

TOGO • 1 q; "Togo Hinterland”; 12 Jun. 1889; E. Kling leg.; ZMB • 2 우; same collection data as for preceding; ZMB. - Centrale Region • 1 +; Bismarckburg [near Yégué]; [ $8^{\circ} 10^{\prime} 32.74^{\prime \prime} \mathrm{N}, 0^{\circ} 41^{\prime} 09.42^{\prime \prime} \mathrm{E}$ ]; 21-22 Apr. 1893; L. Conradt leg.; ZMB • 1 क; same collection data as for preceding but 28 May-12 Jun. 1893; ZMB • 3 q 9 ; same collection data as for preceding but 30 Jun.-3 Jul. 1893; ZMB • 1 क; same collection data as for preceding; ZMB. - Plateaux Region • 1 + ; Misahöhe; [6 $6^{\circ} 57^{\prime}$ N, 0 0 35' E]; 16 Jun 1894; E. Baumann leg.; ZMB.

\section{Description}

Female

Size. $26.6-31.7 \mathrm{~mm}$.

COLOR. Black except for the following, which are ferruginous: basal half of mandible, lower part of clypeus (in most specimens), scape ventrally, foreleg from femur onward except for dark brown stripe ventrally on femur, and mid- and hindlegs from femur onward. Wings fuscous, with greenish or purplegreenish iridescence.

VeSTITURE. Appressed setae on clypeus and paraocular area brassy, on collar, scutum and propodeal enclosure black. Erect setae on clypeus, paraocular area, collar, scutum and propodeal enclosure black. Erect propodeal setae oriented perpendicularly, slightly curved towards anterior. Lower center of clypeus glabrous. Scutellum densely and coarsely pubescent.

StRUCTURE. Free clypeal margin medially with broad notched tooth, stepped above. Clypeus convex, without indentation or carina. Scutellum convex. Metanotum slightly raised, not bituberculate. $2^{\text {nd }}$ recurrent vein joins interstitium between submarginal cells II and III. Propodeal enclosure without any notable ridges. Foretarsomere I 2.9-3.3 $\times$ length of antepenultimate spine. Petiole length $2.2-2.3 \times$ its medial width.

\section{Male}

SizE. $27.4-28.3 \mathrm{~mm}$.

CoLOR. Black except for basal half of mandible, which is ferruginous. The following can be ferruginous as well: apical half of forefemur, basal half of foretibia, apical half of mid- and hindfemur, mid- and hindtibia, and hindtarsomeres I-III. Wings fuscous, with greenish iridescence.

VeSTITURE. Appressed setae on clypeus and paraocular area silvery, on collar, scutum and propodeal enclosure black. Erect setae on clypeus, paraocular area, collar, scutum and propodeal enclosure black. Erect propodeal setae oriented perpendicularly, slightly curved towards anterior. Lower center of clypeus glabrous. Scutellum densely and coarsely pubescent.

STRUCTURE. Free clypeal margin with concavely truncated broad tooth originating behind free margin. Scutellum convex. Metanotum slightly raised, not bituberculate. $2^{\text {nd }}$ recurrent vein joins markedly proximal from interstitium between submarginal cells II and III. Propodeal enclosure without any notable ridges. Posterior margin of metasomal tergum VII convex. Posterior margin of metasomal sternum VII simple, of metasomal sternum VIII triangular. Penis valvae without conspecific modifications. Petiole length 2.0-2.1 $\times$ its medial width. Flagellomeres IV-VI with broad placoids covering their entire length, or flagellomeres V-VI with moderately broad placoids covering their entire length. 


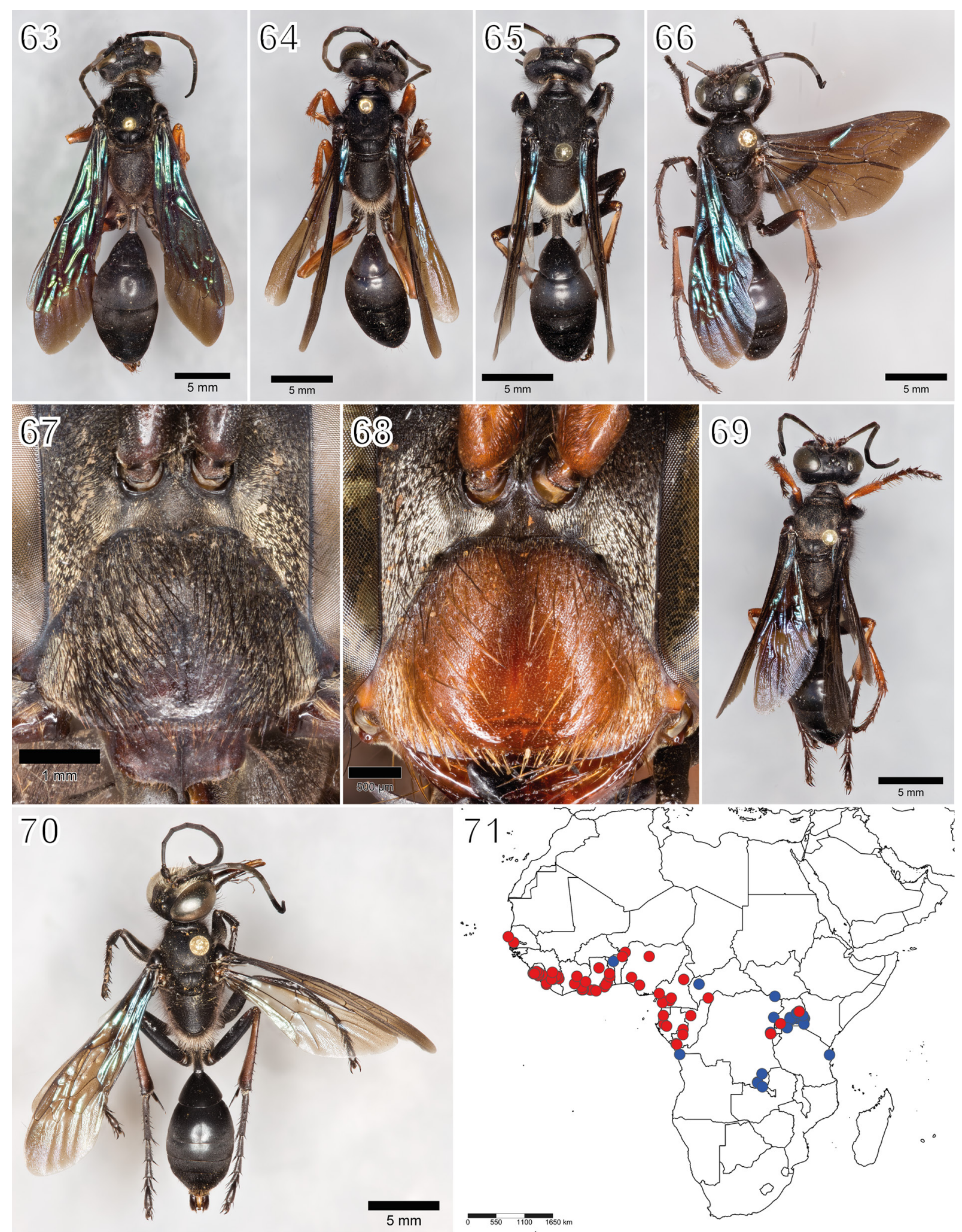

Figs 63-71. 63, 67. Sphex caeruleanus Drury, 1773. 64-65, 68. S. mweruensis (Arnold, 1947). 66. S. hades sp. nov. 69-70. S. haemorrhoidalis Fabricius, 1781. 63-64, 69. Habitus of 9 . 65-66, 70. Habitus of 7 . 67-68. Frontal view of $q$. 71. Geographic distribution of $S$. haemorrhoidalis (red) and S. victoria sp. nov. (blue). 


\section{Variation}

Males of S. caeruleanus seem to be highly variable regarding leg coloration and placoid pattern. The midleg can be ferruginous from the apical half of the femur onwards, with the hindfemur, hindtibia and first few tarsomeres also being ferruginous. Other specimens have only the inner side of the hindtibia dark ferruginous, or all legs black. Broad placoids can be present on flagellomeres IV-VI or V-VI, or narrow placoids on $\mathrm{V}-\mathrm{VI}$.

\section{Distribution}

Western to central Africa.

\section{Remarks}

Unfortunately, the holotype of $S$. caeruleanus could not be located and has probably been destroyed. The original description of this species is naturally quite vague, and it does not mention the conspicuous greenish iridescence which is present on the wings. However, Menke in Bohart \& Menke (1976) synonymized S. caeruleanus with S. pulchripennis, and we do not feel that there is enough evidence to disprove his assumption.

Regarding the identity of S. pulchripennis, we are confident that our interpretation of the species matches that of the original author. The description mentions the female clypeus being ferruginous at the apex and the wings shining green, the combination of which is unique among the African Sphex. Furthermore, the stated body length of $27 \mathrm{~mm}$ exceeds that of the other members of the group.

Sphex hades sp. nov.

urn:1sid:zoobank.org:act:6F6FF34B-032A-457F-A32A-1B83A1337A51

Figs 62 (purple), 66

\section{Differential diagnosis}

What separates this species from all other African Sphex is the fact that its males have the appressed clypeal setae black. Regarding other characters, $S$. hades sp. nov. (Fig. 66) resembles S. victoria sp. nov. (Fig. 74) in having the hindtibia and a substantial part of the mandible ferruginous, and the erect mesosomal setae as well as the wings being dark.

\section{Etymology}

'Hades' is an Ancient Greek noun in apposition and the name of the mythological Greek god of the underworld.

\section{Material examined}

\section{Holotype}

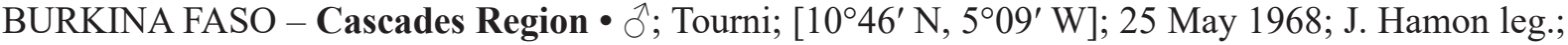
MNHN.

\section{Paratypes}

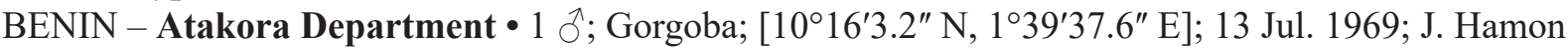
leg.; MNHN.

BURKINA FASO - Cascades Region $\bullet 1$; same collection data as for holotype; MNHN. - Centre-

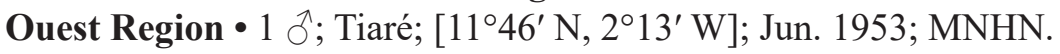

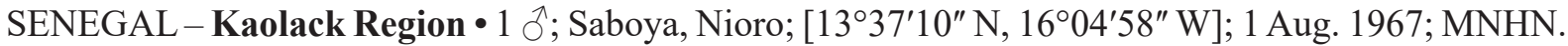




\section{Description}

Female

Unknown.

\section{Male}

Size. $19.5-22.6 \mathrm{~mm}$.

COLOR. Black except for the following, which are ferruginous: basal half of mandible and hindtibia. Wings fuscous, with cyan-violet iridescence.

VeSTITURE. Appressed setae on clypeus, collar, scutum and propodeal enclosure black, on paraocular area silvery. Erect setae on clypeus, paraocular area, collar, scutum and propodeal enclosure black. Erect propodeal setae oriented perpendicularly, slightly curved towards anterior. Lower center of clypeus glabrous. Scutellum densely and coarsely pubescent.

STRUCTURE. Free clypeal margin medially with tooth emerging from inner side. Scutellum convex. Metanotum slightly raised, not notably bituberculate. $2^{\text {nd }}$ recurrent vein joins slightly proximal from interstitium between submarginal cells II and III. Propodeal enclosure without any notable ridges. Posterior margin of metasomal tergum VII convex. Posterior margin of metasomal sternum VII simple, of metasomal sternum VIII triangular. Penis valvae without conspicuous modifications. Petiole length 2.0-2.6 $\times$ its medial width. Flagellomere $\mathrm{V}$ with moderately broad placoid covering its proximal fourfifths, flagellomere VI with moderately broad placoid covering between its proximal fifth and its proximal two-thirds.

\section{Variation}

Unknown.

\section{Distribution}

Western Africa.

Sphex haemorrhoidalis Fabricius, 1781

Figs 34, 69-70, 71 (red)

Sphex haemorrhoidalis Fabricius, 1781: 443, sex not stated [ㅇ].

Sphex nigripes var. volubilis Kohl, 1895: 64, + , $\widehat{~}$. Synonymized with Sphex haemorrhoidalis by R. Turner 1912: 369

\section{Differential diagnosis}

Theoretically, females of S. haemorrhoidalis (Fig. 69) can be recognized through their ferruginousgolden appressed paraocular and lateroclypeal setae (Fig. 34). As this is not always perfectly visible, the combination of a largely ferruginous clypeus and the absence of a silvery luster in the appressed paraocular setae can be used for recognition as well. The silvery luster is present in females of S. victoria sp. nov. (Fig. 35) and in those of S. caeruleanus (Fig. 67) and S. mweruensis (Fig. 68). Females of S. umtalicus (Fig. 33) have at most a small area on the paraocular area with a silvery luster that differs from the basic color of their black appressed paraocular and clypeal setae, and their clypeus is entirely black, with the exception of rare cases that have up to the lower clypeal half ferruginous.

Males (Fig. 70) are identifiable through the combination of at least some of the erect scutal setae being silvery, the clypeus being entirely black and the wings only slightly fuscous. In males of S. victoria sp. nov. (Fig. 74), S. caeruleanus and S. umtalicus, all scutal and propodeal setae are black. While 
the majority of the erect mesosomal setae are silvery in males of $S$. mweruensis (Fig. 65), they differ from those of $S$. haemorrhoidalis in usually having the clypeal margin ferruginous. Furthermore, male S. haemorrhoidalis differ from those of the other species within the group in usually having almost the entire clypeus covered with appressed setae, except the area near the free margin, instead of the glabrous spot being medially at least $15 \%$ of the total clypeal height.

\section{Material examined}

\section{Holotype}

AFRICA $\bullet$ + ; tropical Africa [probably West Africa]; BMNH, coll. Banks.

\section{Other material}

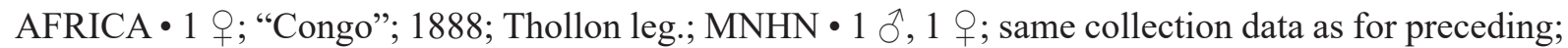
MNHN.

BENIN - Alibori Department • 1 q; Kandi, near Saa; [11 ${ }^{\circ} 07^{\prime} 43^{\prime \prime}$ N, $2^{\circ} 56^{\prime} 13^{\prime \prime}$ E]; Jun. 2001; F. Kantner leg.; THD-020-OOLM; GenBank CO1 gene: MW538572; OÖLM.

CAMEROON • 1 क; 12 Mar. 1892; H. Brauns leg.; TMP • 1 q, paralectotype of Sphex nigripes var. volubilis Kohl, 1895; Schäfer leg.; ZMB • 1 + , paralectotype of Sphex nigripes var. volubilis Kohl, 1895; "Sanaga"; Heim leg.; ZMB. - Adamawa Region • 1 O ; SE of Ngaoundéré, ranch; [ $\left.7^{\circ} 08^{\prime} \mathrm{N}, 13^{\circ} 42^{\prime} \mathrm{E}\right]$; 9-12 May 2006; J. Oehlke leg.; coll. Jacobs. - Centre Region • 1 क; Biyan; [ $3^{\circ} 24^{\prime}$ N, $11^{\circ} 14^{\prime}$ E];

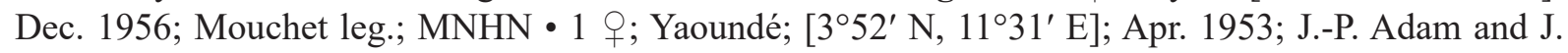
Rageau leg.; MNHN • 2 우; same collection data as for preceding but Oct. 1953; MNHN • 68 q $ᄋ$;

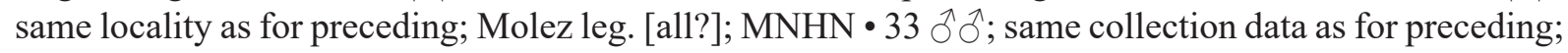

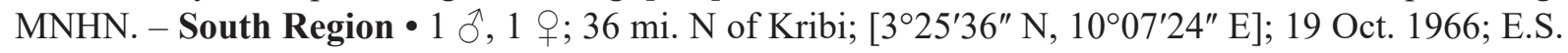
Ross and K. Lorenzen leg.; CAS • 1 क; Longji; [304'40.7" N, 958'29.1" E]; Apr. 1904; H. Paschen leg.; ZMB • 1 क; same collection data as for preceding; ZMB • 1 q; Nkoemvon; [ $\left.2^{\circ} 49^{\prime} \mathrm{N}, 11^{\circ} 08^{\prime} \mathrm{E}\right] ; 13$ Jul.-24 Aug. 1980; D. Jackson leg.; BMNH. - Southwest Region • 1 §, lectotype of Sphex nigripes var. volubilis Kohl, 1895 (designated here); Barombi Station; P. Preuss leg.; ZMB • 4 o $\odot$, paralectotypes of Sphex nigripes var. volubilis Kohl, 1895; same collection data as for lectotype of Sphex nigripes var. volubilis Kohl, 1895; ZMB.

CENTRAL AFRICAN REPUBLIC • 1 \%; M. Boulard leg.; MNHN. - Lobaye • 1 q; La Maboke [near M'Baiki]; [353' N, 1800' E]; 21 Aug. 1969; P. Teocchi leg.; MNHN.

ETHIOPIA - Amhara Region • 1 क; Jowaha, N of Debra Sina; 7 Aug. 1946; K.M. Guichard leg.; BMNH.

GABON - Estuaire Province • $1 \hat{\partial}, 1$ क; Crystal Mountains, Komo; [0 $\left.0^{\circ} 49^{\prime} 45.6^{\prime \prime} \mathrm{N}, 10^{\circ} 08^{\prime} 5.7^{\prime \prime} \mathrm{E}\right]$; 1-15 Oct. 1969; A. Villiers leg.; MNHN • 1 o $^{\top}$; Crystal Mountains, Muni; [049'45.6" N, $\left.10^{\circ} 08^{\prime} 5.7^{\prime \prime} \mathrm{E}\right]$;

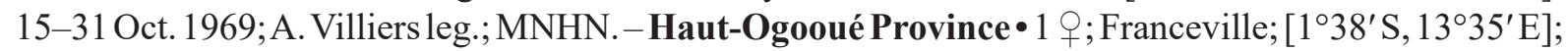
1888; P.S. de Brazza leg.; MNHN. - Moyen-Ogooué Province • 1 q; Lambaréné; [ $0^{\circ} 41^{\prime} 18^{\prime \prime}$ S,

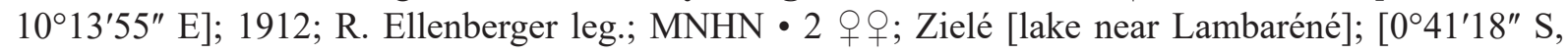
10¹3'55" E]; 22 May 1993; MNHN. - Ngounié Province • 1 q, paralectotype of Sphex nigripes var. volubilis Kohl, 1895; "Chûtes de Samlia, Rivière N'Gami” [actually chute de Samba, Ngounié River]; A. Mocquerys leg.; IRSN.

GHANA - Eastern Region - 1 \%; 6 km E of Akosombo; $6^{\circ} 15^{\prime}$ N, $0^{\circ} 05^{\prime}$ E; 28 Aug. 2012; M. and J. Schwarz leg.; OÖLM • 1 \%; same collection data as for preceding but 30 Aug. 2012; THD-034-OOLM;

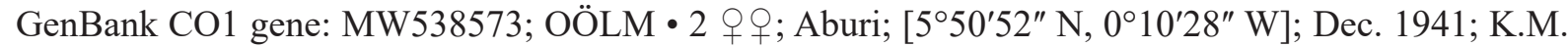
Guichard leg.; BMNH $\bullet 2$ ô $\widehat{\text {; }}$; same collection data as for preceding; BMNH. - Savannah Region •

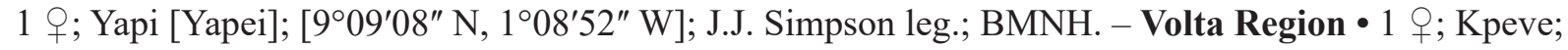


[6²1'20" N, 0²0'01" E]; Jun. 1942; K.M. Guichard leg.; BMNH. - Western Region • 1 q; Ankasa

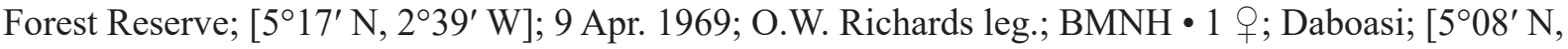

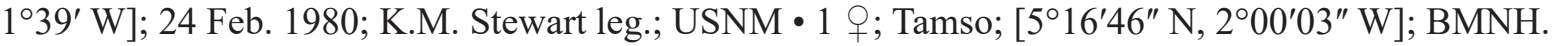

GUINEA • 1 +; Westermann leg.; ZMB. - Nzérékoré Region • 1 đ’; near Diéké; [7²0'54" N, 857'15" W]; 8-9 Feb. 2003; J. Oehlke leg.; coll. Jacobs • 1 q; Kéoulenta; [740’46" N, 8¹8'51" W]; 2 Jun. 1942; M. Lamotte leg.; MNHN • 1 क; Nzo; [740'46" N, 8¹8'51" W]; 2 Jun. 1942; M. Lamotte leg.; MNHN •

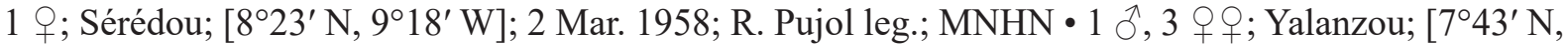
$\left.8^{\circ} 41^{\prime} \mathrm{W}\right]$; 2 Jun. 1942; M. Lamotte leg.; MNHN.

KENYA 1 1 ; ; valley of Upper Nzoia River, N of Kavirondo; 5-7 Jun. 1911; S.A. Neave leg.; MNHN.

LIBERIA • 2 q ; Dingano; 10 Aug. 1953; W. Peters leg.; BMNH • 1 क; Varh; 3 Aug. 1953; W. Peters

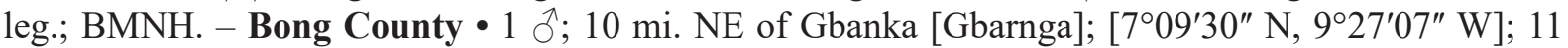

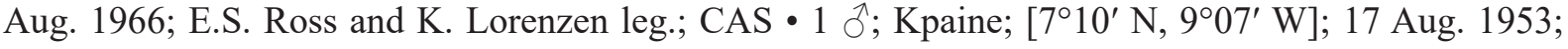
W. Peters leg.; BMNH • 1 क; Kpaine; [ $7^{\circ} 10^{\prime}$ N, $9^{\circ} 07^{\prime}$ W]; 17 Aug. 1953; W. Peters leg.; BMNH. Margibi County • 1 o $^{\prime}$; Harbel, Firestone Plantation; [ $\left.6^{\circ} 17^{\prime} \mathrm{N}, 10^{\circ} 21^{\prime} \mathrm{W}\right]$; 27 Jul. 1966; E.S. Ross and

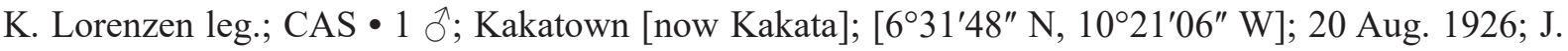
Bequaert leg.; MRAC.

NIGER - Dosso Region • 1 क; Gaya; [1153'03" N, 3²6'57" E]; 17 Oct. 1976; BMNH.

NIGERIA • 1 ○̊; Koya; Jan. 1975; J.T. Medler leg.; BMNH. - Kaduna State • 1 + ; Samaru; [1109'39" N, 7³8'44" E]; 25 Aug. 1970; Ward leg.; BMNH • 1 क; same collection data as for preceding but 4 Sep. 1970; BMNH • 1 क; Samaru, Fadama; [11 09'39" N, 7³8'44" E]; 15 Jul. 1970; BMNH. - Osun State • 1 ठ; Ile-Ife; [7²8'56" N, 4³3'37" E]; 6 Mar. 1975; J.T. Medler leg.; BMNH.

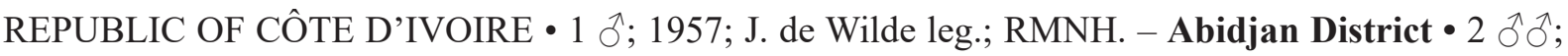
Adiopodoumé; [5²0'29.2" N, 407'59.2" W]; 1967; P.J. Hummelen leg.; RMNH • 1 ö; same locality as for preceding; 30 Aug. 1962; J. von Dinther leg.; RMNH • 1 §ో; same locality as for preceding; 17 Nov. 1967; Gillon leg.; MNHN • 1 क; Banco National Park; [5²3' N, 403' W]; R. Paulian and C. Delamare

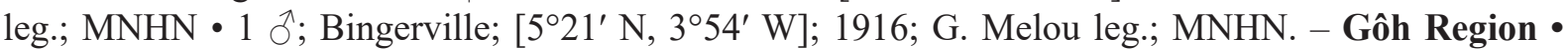

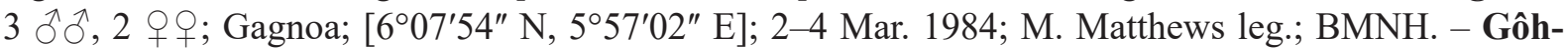
Djiboua District • 1 क; Oumé; [ $6^{\circ} 23^{\prime}$ N, $5^{\circ} 25^{\prime}$ W]; 20 Jan. 1991; W.J. Pulawski leg.; CAS. - Indénié-

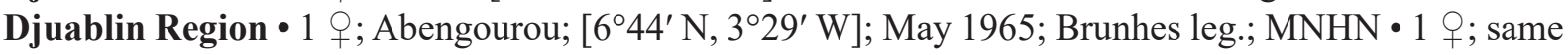
collection data as for preceding but Jun. 1965; MNHN. - Lagunes District • 1 \%; $40 \mathrm{~km} \mathrm{NW}$ of Abidjan; [546'27" N, 407'34" W]; 6 Jan. 1991; W.J. Pulawski leg.; CAS • 1 क; Nidiza [N'Douci or Nzida?];

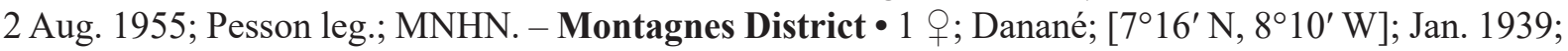
L. Chopard leg.; MNHN • 1 q; same locality as for preceding; Dec. 1930-Apr. 1931; Ch. Alluaud and P.A. Chappuis leg.; MNHN. - Vallée du Bandama District • 1 + ; Bouaké; [ $\left.7^{\circ} 41^{\prime} \mathrm{N}, 5^{\circ} 01^{\prime} \mathrm{W}\right]$; 1977; P.M. Elsen leg.; MRAC.

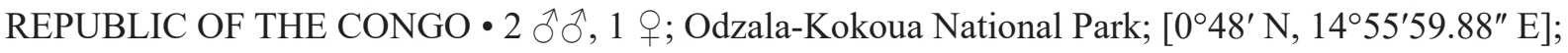
Oct. 1963; A. Descarpentries and A. Villiers leg.; MNHN. - Kouilou Department • 1 §; Dimonika, Makaba; [407'59" S, 12²1' E]; 28 Feb. 1978; J.-J. Menier leg.; MNHN • 1 O; Mvouti; [4¹4'47" S, $12^{\circ} 29^{\prime 2} 0^{\prime \prime}$ E]; 17 Feb. 1978; J.J. Menier leg.; MNHN. - Lékoumou Department • 1 +; Mbouloupambou; [2³2'26.16" S, 13³2'45.96" E]; 22 Apr. 1980; C. Morin leg.; MNHN.

RWANDA - Western Province • 1 q; Ishwa, Lake Albert; [2²3'04" S, 28 55'58” E]; Sep. 1935; H.J. Brédo leg.; MRAC. 


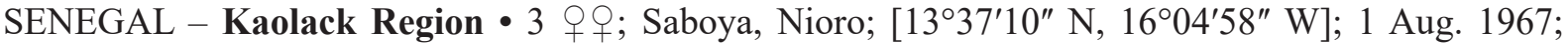

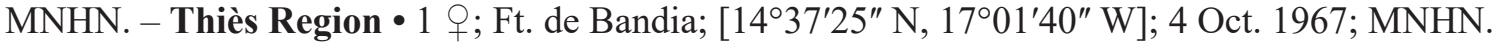

SIERRA LEONE • 1 q; W.G. Clements leg.; BMNH • 1 đે; ZMB • 1 क; Dumballa; 7 Sep. 1912; J.J. Simpson leg.; BMNH • 1 क; Yorke; 30 Sep. 1912; J.J. Simpson leg.; BMNH. - Eastern Province • 2 q $q$;

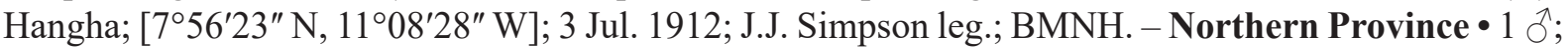
Kumrabai; [8³2'02" N, 1205'33" W]; 21 Sep. 1912; J.J. Simpson leg.; BMNH. - Southern Province •

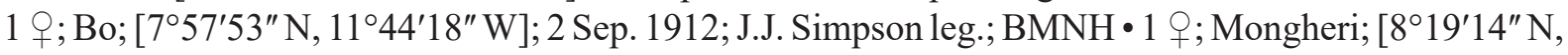

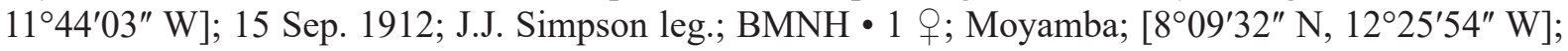

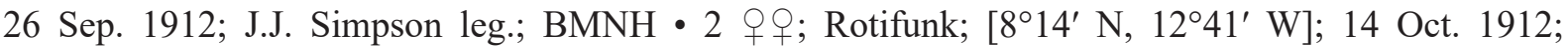

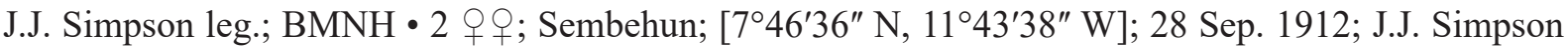

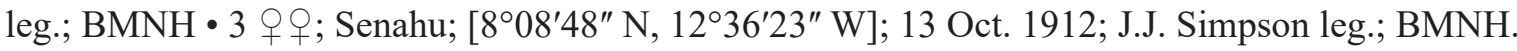

TOGO $\bullet 2$ 우, paralectotypes of Sphex nigripes var. volubilis Kohl, 1895; “Togo Hinterland”; 8 and 15 May 1889; E. Kling leg.; ZMB. - Centrale Region • 1 + , paralectotype of Sphex nigripes var. volubilis Kohl, 1895; Bismarckburg (near Yégué); 30 Jun.-3 Jul. 1893; L. Conradt leg.; ZMB. - Plateaux Region •

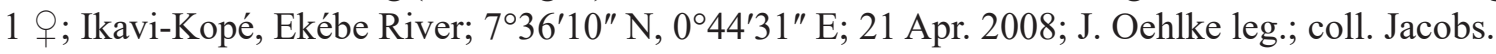

UGANDA - Eastern Region • 1 क; Mbale-Kumi Road, $3700 \mathrm{ft} \mathrm{S}$ of Lake Salisbury; [1 ${ }^{\circ} 33^{\prime} 33^{\prime \prime}$ N, 3357'15" E]; 15-17 Aug. 1911; S.A. Neave leg.; MNHN. - Western Region • 1 क; Fort Portal Road, Mbarara, Southern Toro ; [0³6'48" S, 30³9'30" E]; 22-24 Oct. 1911; S.A. Neave leg.; MNHN.

\section{Description}

Female

Size. 24.3-24.9 mm.

CoLoR. Black except for the following, which are ferruginous: basal half of mandible, clypeus, scape, pedicel, legs from apical fourth of femur onward excluding apical half of claw and apical metasomal segment. Wings fuscous, with violet-bluish iridescence.

VeSTITURE. Appressed setae on clypeus and paraocular area ferruginous-golden, on collar, scutum and propodeal enclosure black. Erect setae on clypeus, paraocular area, collar, scutum and propodeal enclosure black. Erect propodeal setae oriented perpendicularly, slightly curved towards anterior. Lower center of clypeus glabrous. Scutellum densely and coarsely pubescent.

STRUCTURE. Free clypeal margin medially with broad, notched tooth, stepped above. Clypeus without distinct indentation or carina. Scutellum convex. Metanotum slightly raised, not notably bituberculate. $2^{\text {nd }}$ recurrent vein joins interstitium between submarginal cells II and III. Propodeal enclosure without any notable ridges. Foretarsomere I 2.9-3.3 $\times$ length of antepenultimate spine. Petiole length $2.5-2.6 \times$ its medial width.

\section{Male}

SIZE. $19.2-23.5 \mathrm{~mm}$.

CoLOR. Black except for basal half of mandible and hindtibia, which are ferruginous. Fore- and hindwing slightly, their apical margin more conspicuously fuscous, forewing with violet iridescence.

Vestiture. Appressed setae on clypeus and paraocular area silvery, on collar black, on scutum and propodeal enclosure silvery or black. Erect setae on clypeus, paraocular area and collar black, on scutum usually silvery, on propodeal enclosure black or sometimes silvery. Erect propodeal setae oriented 
perpendicularly, slightly curved towards anterior. Clypeus glabrous only near free margin. Scutellum densely and coarsely pubescent.

StRUCTURE. Free clypeal margin medially with tooth emerging from inner side. Scutellum convex. Metanotum slightly raised, not notably bituberculate. $2^{\text {nd }}$ recurrent vein joins slightly proximal from interstitium between submarginal cells II and III. Propodeal enclosure without any notable ridges. Posterior margin of metasomal tergum VII convex. Posterior margin of metasomal sternum VII simple, of metasomal sternum VIII triangular. Petiole length $2.1-2.4 \times$ its medial width. Flagellomere V with narrow placoid covering its proximal four-fifths.

\section{Variation}

The female clypeus, flagellum, tarsi and metasomal apex can be ferruginous or black to a varying extent. In males, the erect scutal and propodeal setae are silvery or black to a varying extent, though there are always at least some silvery scutal setae.

\section{Distribution}

Western to central Africa.

\section{Remarks}

Kohl did not properly label the types of $S$. nigripes var. volubilis, and they are only indicated by the locality notes in the original description, where twelve specimens from four more or less well-defined areas are mentioned (Cameroon, Togo Hinterland, "Chûtes de Samlia", "Grand Bana"). We were able to find a few specimens from matching regions that have identification labels by Kohl, and in conjunction with the remaining specimens of this species in the ZMB from the same series, or other specimens there that were collected before 1895 in Cameroon or Togo, their number amounts exactly to ten. There is only one specimen from the type series (from "Chûtes de Samlia") in the IRSN, and under the assumption that there was only one from "Grand Bana" as well (the specimen was thought to be deposited in the NHMW, but is apparently not there), those ten ZMB specimens would likely constitute the remainder of the syntypes.

Sphex mweruensis (Arnold, 1947)

Figs 62 (yellow), 64-65, 68

Chlorion haemorrhoidalis var. mweruensis Arnold, 1947: 146, ๆ, ô.

\section{Differential diagnosis}

Females of this species are easy to recognize, as they are the only ones within the umtalicus group that have silvery erect setae on the posterior propodeal margin (Fig. 64). In males, the extent of the dorsal silvery setae is greater, as they cover all of the propodeal enclosure and occasionally parts of the scutum as well (Fig. 65). Males of $S$. haemorrhoidalis (Fig. 70) can be very similar, but always have the free clypeal margin black and only slightly concave, and the appressed setae barely leave a glabrous spot on the clypeus. Those of $S$. mweruensis have a glabrous spot in the lower clypeal center, the free margin is often (though not always) ferruginous and has a gentle but conspicuous concave emargination on either side of the medial process, which is apically concave itself. Males of $S$. caeruleanus have the free clypeal margin and the process shaped similarly, but the process is approximately twice as wide. Moreover, they are easily distinguished through their uniformly black erect propodeal setae.

\section{Material examined}

Lectotype (designated here)

ZAMBIA• • +; "Seremani Mweru”; 27 Jan. 1944; IRSN. 


\author{
Paralectotype \\ ZAMBIA - Northern Province • 1 đ̊; Mpulungu; 2 May 1944; IRSN.
}

\title{
Other material
}

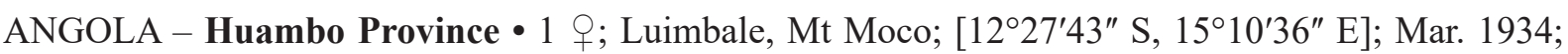
BMNH.

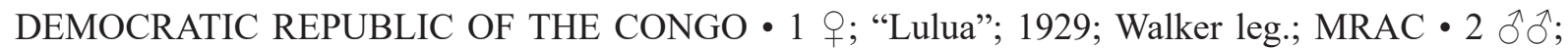
"Lulua: Juele River"; 1929; Walker leg.; MRAC • 2 o o ; Kibombo; Oct. 1930; H.J. Brédo leg.; MRAC. - Haut-Katanga • 1 \%; Elisabethville [now Lubumbashi]; [11 $40^{\prime}$ S, $27^{\circ} 29^{\prime}$ E]; Jan. 1938; H.-J. Brédo leg.; IRSN $\bullet 1 \delta^{\lambda}$; same collection data as for preceding but 10 Jan. 1938 ; IRSN $\bullet 1$; ; same locality as

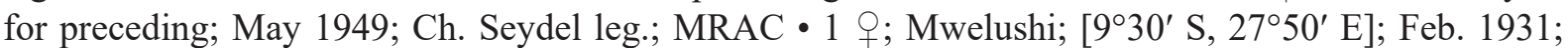

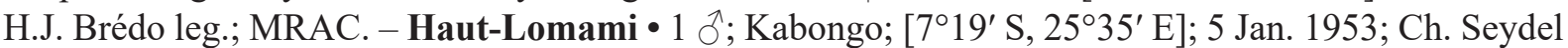

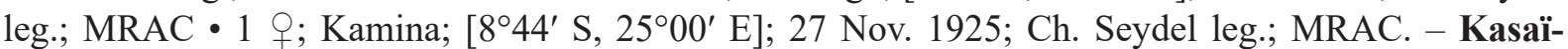

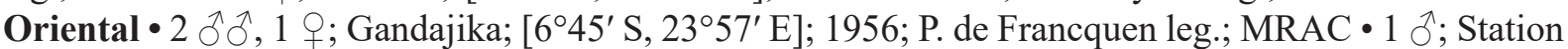

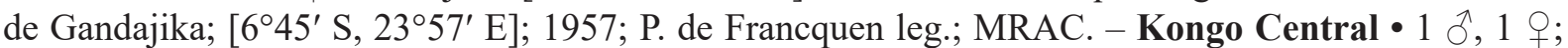

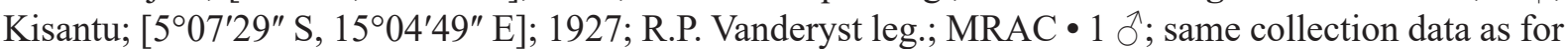

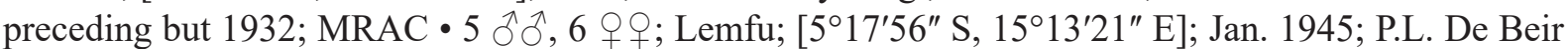
leg.; MRAC • 1 \&; same collection data as for preceding but May 1945; MRAC • 2 우; same collection data as for preceding but Jun. 1945; MRAC $\bullet 2 \widehat{\partial}, 5$ + \% ; same collection data as for preceding but Dec.

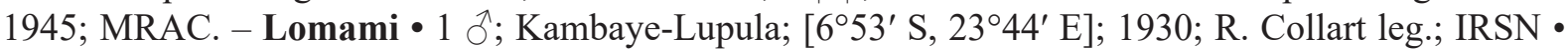

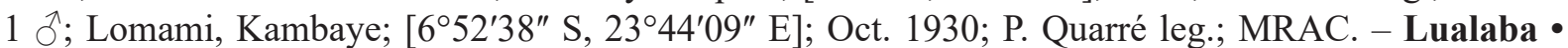

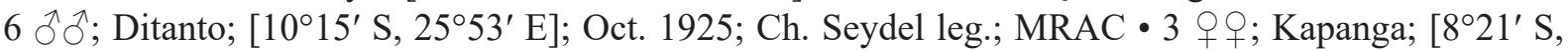
$22^{\circ} 34^{\prime}$ E]; Oct. 1932; F.G. Overlaet leg.; MRAC 1 ( ${ }^{\lambda}$; same collection data as for preceding but Dec. 1932; MRAC.

MALAWI - Southern Region • 1 क; Mlanje; [1601'53" S, 35³0' E]; 11 Dec. 1913; S.A. Neave leg.; BMNH.

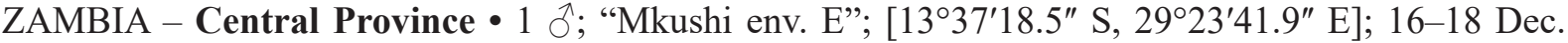
2004; M. Snižek leg.; OÖLM. - Northern Province • 3 q o+ ; Kaputa; [8²8' S, 2940' E]; 8 Jan. 1944;

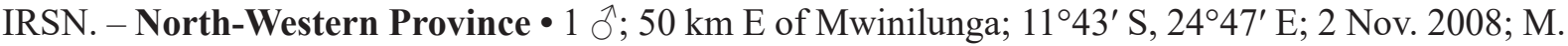
Halada leg.; THD-019-OOLM; GenBank CO1 gene: MW538574; OÖLM.

\section{Description}

Female

Size. 22.2-24.5 mm.

CoLOR. Black except for the following, which are ferruginous: basal half of mandible, clypeus, scape, pedicel, foreleg from apical half of femur, and mid- and hindlegs from femur onward excluding apical half of claw. Wings fuscous, with violet luster.

VestiTURE. Appressed setae on clypeus silvery-golden laterally, black otherwise, on paraocular area silvery, on collar, scutum and propodeal enclosure black. Erect setae on clypeus ferruginous laterally, black otherwise, on paraocular area, collar, scutum and propodeal enclosure black, on posterior margin of propodeum silvery. Erect propodeal setae oriented perpendicularly, slightly curved towards anterior. Central third of clypeus glabrous. Scutellum densely and coarsely pubescent.

STRUCTURE. Free clypeal margin medially with broad, notched tooth, slightly stepped above. Clypeus without indentation or carina. Scutellum convex. Metanotum slightly raised, not notably bituberculate. $2^{\text {nd }}$ recurrent vein nearly joins interstitium between submarginal cells II and III. Propodeal enclosure 
without any notable ridges. Foretarsomere I $2.7-3.4 \times$ length of antepenultimate spine. Petiole length $1.9-2.8 \times$ its medial width.

Male

Size. 21.5-24.8 mm.

CoLor. Black except for the following, which are ferruginous: basal half of mandible, free clypeal margin, midtibia anteriorly, midtarsomere I, hindtibia, hindtarsomeres I-II and basal half of claw. Wings fuscous, forewing with violet luster.

VeSTITURE. Appressed setae on clypeus and paraocular area silvery, on collar, scutum and propodeal enclosure black. Erect setae on clypeus, paraocular area and collar black, on scutum black or black and silvery, on propodeal enclosure silvery. Erect propodeal setae oriented perpendicularly, slightly curved towards anterior. Lower center of clypeus glabrous. Scutellum densely and coarsely pubescent.

STRUCTURE. Free clypeal margin medially with tooth emerging from inner side, and distinctly concave next to process. Scutellum convex. Metanotum slightly raised, not notably bituberculate. $2^{\text {nd }}$ recurrent vein joins slightly proximal from interstitium between submarginal cells II and III. Propodeal enclosure without any notable ridges. Posterior margin of metasomal tergum VII convex. Posterior margin of metasomal sternum VII simple, of metasomal sternum VIII triangular. Penis valvae without conspicuous modifications. Petiole length 2.1-2.5 $\times$ its medial width. Flagellomeres IV-VI with broad placoids covering their entire length.

\section{Variation}

In some males, the free margin can be black instead of ferruginous, the erect scutal setae can be uniformly black or partially silvery, and the propodeal enclosure can have erect black setae as well.

\section{Distribution}

Central Africa.

\section{Remarks}

The two specimens we consider part of the type series that are owned by the IRSN had both been labeled "paratype". We believe the labels were added later, possibly by Leclercq, and do not constitute an official designation by Arnold. Since we could not locate any publication that designates a lectotype for either of these two species, we treat them as syntypes and designate a lectotype here.

Sphex umtalicus Strand, 1916

Figs 3-4, 27, 33, 72, 77 (red)

Sphex umtalicus Strand, 1916: 105, đ̃.

Sphex nigripes var. pachyderma Strand, 1916: 106,, . Syn. nov.

Chlorion haemorrhoidalis var. kobrowi Arnold, 1928: 367, $q$ (holotype: + , South Africa, Gauteng, Johannesburg, TMP, not examined). Syn. nov.

Chlorion haemorrhoidalis var. basuto Arnold, 1947: 146, sex not stated (holotype or syntypes: Lesotho,

Berea District, Mamathes, SAM, not examined). Syn. nov.

\section{Differential diagnosis}

In most cases, specimens of $S$. umtalicus are easily identifiable within the species group through their black clypeus, uniformly black legs and fuscous wings with cyan-violet iridescence (Fig. 72). However, we observed intraspecific variations in this species as well as in $S$. haemorrhoidalis that can cause 
confusion between the two of them. Females of $S$. haemorrhoidalis normally have the clypeus ferruginous (Fig. 34), though it can also be black to a varying extent, sometimes almost completely. While the tibiae and part of the femora and tarsi are always ferruginous in female S. haemorrhoidalis (Fig. 69) and S. victoria sp. nov. (Fig. 73), a southern African population of S. umtalicus (previously described as var. basuto/kobrowi) also has these areas ferruginous, as well as the lower part of the female clypeus. The best way to differentiate between these females is the color of the appressed clypeal and paraocular setae, which is always dark brown in S. umtalicus and can have a slight silvery shimmer on the paraocular area (Fig. 33). The appressed clypeal setae of female $S$. haemorrhoidalis are inconspicuous, but the appressed lateroclypeal and paraocular setae have a ferruginous-golden shimmer (Fig. 34).

Males of the southern African population of S. umtalicus are characterized by having the wings mostly hyaline and all tibiae ferruginous, whereas those of the other species in the group have only the hindtibia ferruginous. Like all males of the species, they have silvery appressed clypeal setae and uniformly black erect mesosomal setae.

\section{Material examined}

\section{Holotype}

ZIMBABWE - Manicaland• ○’; Umtali [now Mutare]; A. Bodong leg.; DEI.

\section{Other material}

AFRICA • 1 क; "Afr. or. All. de"; MNHN • 1 \%; "East Africa"; IRSN • 1 +; "Eersteleng"; BMNH •

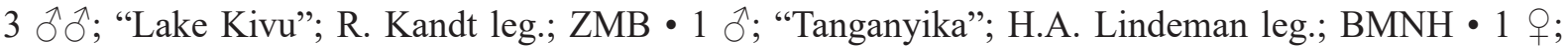
German East Africa; 1912; MNHN・1 $\%$; same collection data as for preceding; MNHN.

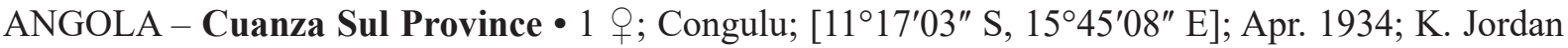
leg.; BMNH.

DEMOCRATIC REPUBLIC OF THE CONGO - Haut-Katanga • 1 q; Sakania; [12 $45^{\prime}$ S, 28 $8^{\circ} 33^{\prime} 36^{\prime \prime}$ E]; 15 Mar. 1938; IRSN • 1 क; Sakania (marais Lukabungu); [1245’ S, $28^{\circ} 34^{\prime}$ E]; 15 Mar. 1938; H.J. Brédo leg.; MRAC • 1 क ; Tera; [1208' S, 28³4' E]; Feb. 1948; R. Mouchamps leg.; MRAC. - Haut-Lomami • $1 \partial^{\top}$; "Upemba National Park, Kankunda (rive dr. Lupiala)"; [8³0' S, 26³0' E]; alt. 1300 m; 13-27 Nov.

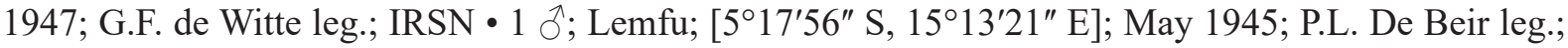
MRAC 1 1 ; Thysville [now Mbanza-Ngungu]; $5^{\circ} 30^{\prime}$ S, $15^{\circ} 00^{\prime}$ E; 2 Jun. 1915; Lang and Chapin leg.; AMNH. - Kongo Central Province $\bullet 2 \widehat{\jmath}$; Mayumbe; Nov. 1938; IRSN.

ETHIOPIA - Amhara Region • 1 đ̃ ; Jowaha, N of Debra Sina; 7 Aug. 1946; K.M. Guichard leg.; BMNH.

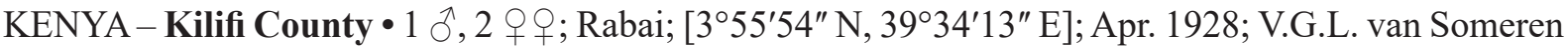
leg.; BMNH. - Kwale County • $3 \delta^{\top} \delta^{\top}$; Funzi Island, near Funzi workshop; $4.57749^{\circ} \mathrm{S}, 39.43825^{\circ} \mathrm{E}$; 4-10 Jul. 2012; ICIPE/NMK leg.; ICIPE • 1 क; Funzi Island, near Mliani; $4.53577^{\circ} \mathrm{S}, 39.46042^{\circ} \mathrm{E}$; 6 Jul. 2012; ICIPE/NMK leg.; ICIPE • 1 q; Funzi Island, near swimming pool; $4.58463^{\circ} \mathrm{S}, 39.43916^{\circ} \mathrm{E}$;

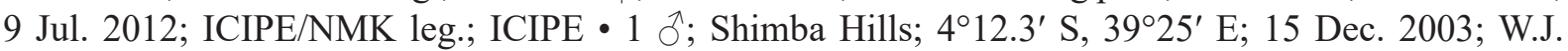

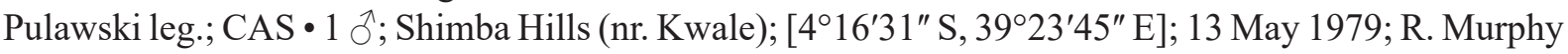
leg.; BMNH • 1 गं; Tiwi Beaches, ca 17 km S of Mombasa; 4¹4.4’ S, 39³6.2' E; 24-26 Jul. 1999; W.J. Pulawski and J.S. Schweikert leg.; CAS. - Makueni County 1 o'; Makueni, Ukamba; $\left[1^{\circ} 48^{\prime}\right.$ S, 37³7’ E]; May 1947; V.G.L. van Someren leg.; BMNH. - Nairobi County • 1 क ; near Nairobi, Vte. de Pongins et Cte de Lambertye; $\left[1^{\circ} 17^{\prime} \mathrm{S}, 36^{\circ} 49^{\prime} \mathrm{E}\right]$; MNHN. - Taita-Taveta County 4 q $q$; Kasigau;

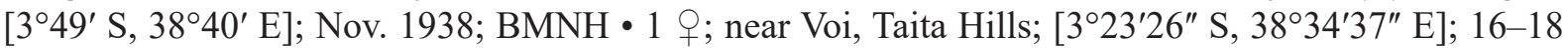
Nov. 1921; H.E. Box leg.; BMNH • 1 क; Voi; [3²3'26" S, 38³4'37" E]; 23 Nov. 1997; M. Snižek leg.; OÖLM • 3 우; Wundanyi; [3²3'54" S, 38²1'37" E]; 19-21 Nov. 1996; Mi. Halada leg.; OÖLM • 


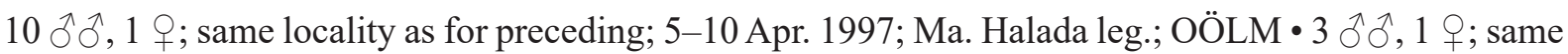
collection data as for preceding but 18-22 Mar. 1997; OÖLM.

LESOTHO - Berea District • 1 q; Mamathes; [290' S, 2751' E]; 28 Dec. 1946; C.F. Jacot-Guillarmod leg.; BMNH・1 1 ; same collection data as for preceding but 1 Jan. 1947; BMNH.

MALAWI - 1 ; NW shore of Lake Nyasa, between Florence Bay and Karonga; 30 Jun.-6 Jul. 1910;

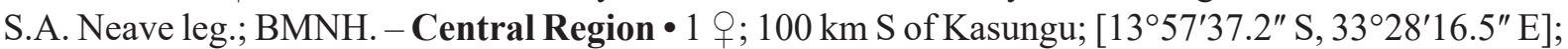

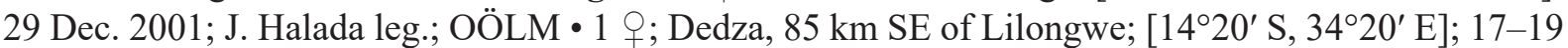

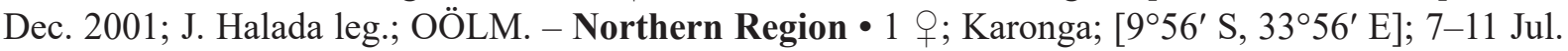
1910; S.A. Neave leg.; BMNH. - Southern Region - 1 \%; Mlanje; $\left[16^{\circ} 01^{\prime} 53^{\prime \prime}\right.$ S, 35³0' E]; 23 Jan. 1912; S.A. Neave leg.; BMNH 2 § ${ }^{\lambda}$; same collection data as for preceding but 4 Dec. 1912; BMNH • 2 of; same collection data as for preceding but 6 Dec. 1912; BMNH $\bullet 1$ \% ; same collection data as for preceding but 11 Dec. 1912; BMNH 11 ; ; same collection data as for preceding but 12 Dec. 1912;

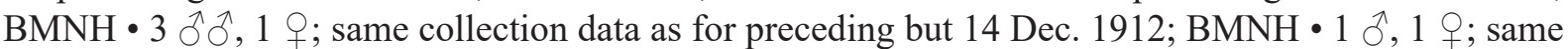
collection data as for preceding but 23 Dec. 1912; BMNH $\bullet 1$ ग; same collection data as for preceding

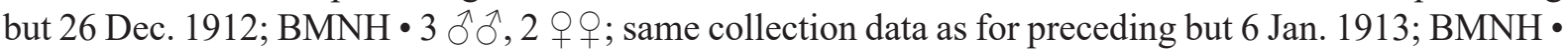

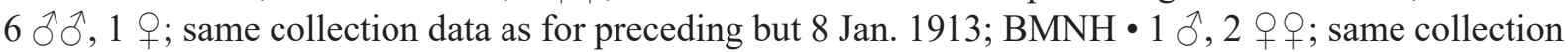
data as for preceding but 13 Jan. 1913; BMNH 1 J ; same collection data as for preceding but 14

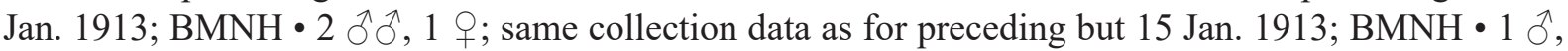

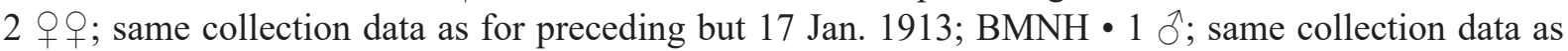
for preceding but 17 Jan. 1913; BMNH 1 \% ; same collection data as for preceding but 21 Jan. 1913;

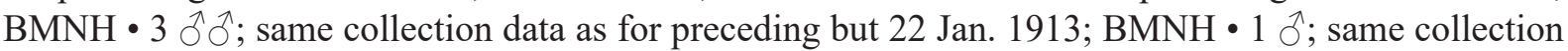
data as for preceding but 24 Jan. 1913; BMNH • 1 ô, 2 q 9 ; same collection data as for preceding but 27 Jan. 1913; BMNH • 1 q; same collection data as for preceding but 29 Jan. 1913; BMNH • 1 \%; same collection data as for preceding but $1 \mathrm{Feb} .1913$; BMNH $\bullet 2$ ô, 1 q ; same collection data as for preceding but $4 \mathrm{Feb} .1913$; BMNH $\bullet 1$ ô, 1 q; same collection data as for preceding but 6 Feb. 1913; $\mathrm{BMNH} \bullet 1$ J, 1 q; same collection data as for preceding but $8 \mathrm{Feb} .1913 ; \mathrm{BMNH} \bullet 1$ \%; same collection data as for preceding but $11 \mathrm{Feb} .1913 ; \mathrm{BMNH} \bullet 1 \hat{\jmath}, 1$; ; same collection data as for preceding but 14 Feb. 1913; BMNH • 5 ㅇ; ; same collection data as for preceding but $17 \mathrm{Feb} .1913$; BMNH • 1 ๆ; same collection data as for preceding but $24 \mathrm{Feb} .1913$; BMNH $\bullet 2$ 우; same collection data as for preceding but $27 \mathrm{Feb}$. 1913; BMNH 2 ô, 3 , 3 우; same collection data as for preceding but 3 Mar. 1913; BMNH •

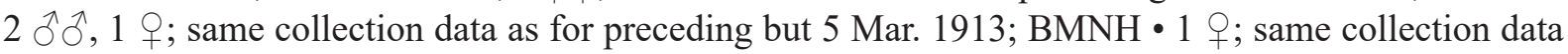
as for preceding but 20 Mar. 1913; BMNH $\bullet 1 \hat{\jmath}, 2$ + $ᄋ$; same collection data as for preceding but 26 Mar. 1913; BMNH • 1 \%; same collection data as for preceding but 17 Apr. 1913; BMNH • 1 \% ; same collection data as for preceding but 10 May 1913; BMNH $\bullet 2$ q $q$; same collection data as for

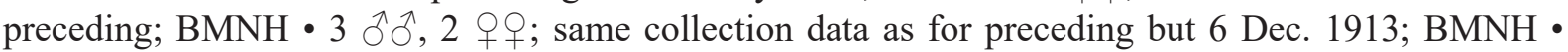

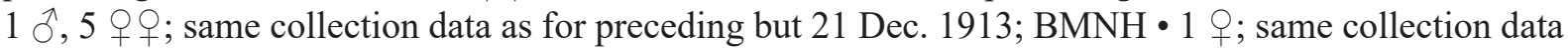
as for preceding but 29 Dec. 1913; BMNH -3 Q 9 ; same collection data as for preceding but 20 Jan.

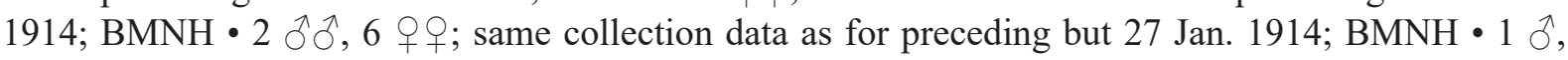
2 qo+; same collection data as for preceding but $10 \mathrm{Feb} .1914$; BMNH $\bullet 2 \widehat{\delta}$; same collection data as for preceding but $13 \mathrm{Feb}$. 1914; BMNH $\bullet 6$ 우; same collection data as for preceding but 16 Feb. 1914;

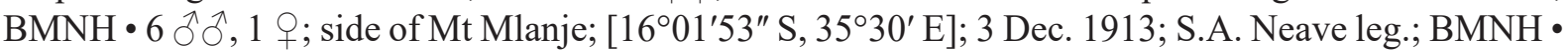
$2 \hat{\jmath}, 1$; ; same collection data as for preceding but 11 Dec. 1913; BMNH $\bullet 1$ \%; side of Mt Mulanje; [16 $\left.01^{\circ} 53^{\prime \prime} \mathrm{S}, 35^{\circ} 30^{\prime} \mathrm{E}\right]$; alt. 3000-4000 ft; 2 Dec. 1913; S.A. Neave leg.; MNHN • 5 우; SW of Lake

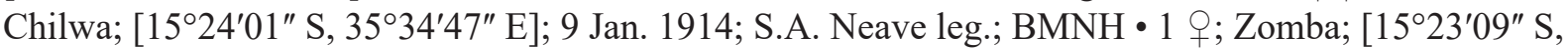
35¹9'07" E]; Nov. 1913; H.S. Stannus leg.; BMNH • 1 q; same collection data as for preceding but Dec. 1913; BMNH • 2 o ; ; same collection data as for preceding but Jan. 1914; BMNH • 1 q; same

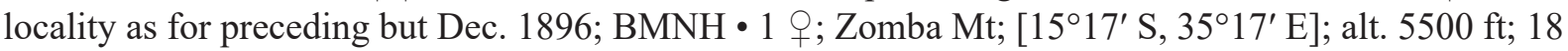
Feb. 1910; S.A. Neave leg.; BMNH. 
MOZAMBIQUE • 1 q; valley of Kola River, near E Mt Chiperone; 3 Apr. 1913; S.A. Neave leg.; BMNH • 1 q ; same collection data as for preceding but 6 Apr. 1913; BMNH $\bullet 2$ o $q$; same collection data as for preceding but 7 Apr. 1913; BMNH. - Manica Province 1 क; $65 \mathrm{~km} \mathrm{~W}$ of Chimoio; [18 $57^{\prime} 51^{\prime \prime} \mathrm{S}$, 32 50'10.1" E]; 10-11 Feb. 2003; J. Halada leg.; OÖLM • 1 क; Amatongas Forest; [19¹1' S,

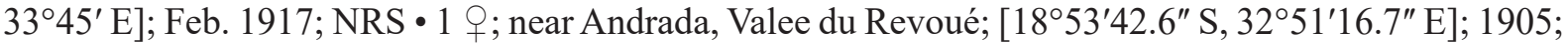

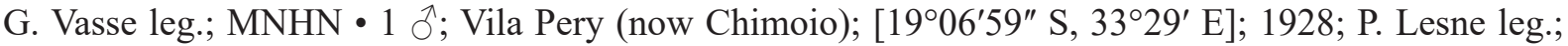

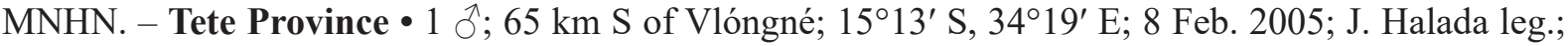

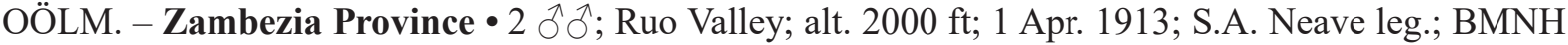
- 1 क; same collection data as for preceding; MNHN $\bullet 1$; ; same collection data as for preceding but 14 Dec. 1913; BMNH.

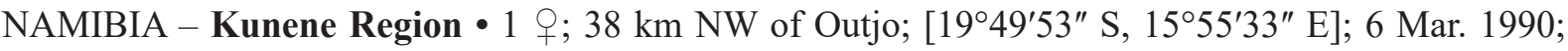

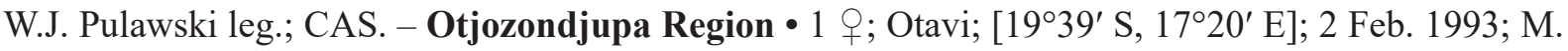
Schwarz leg.; OÖLM.

SOUTH AFRICA • 1 क; Pienaars River; 1898; R. v. Jutrzencka leg.; TMP. - Eastern Cape • 1 \%;

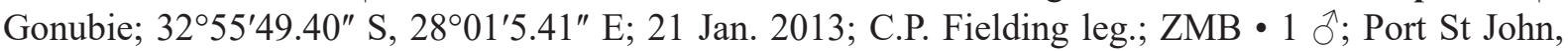

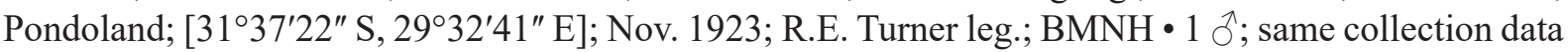
as for preceding but Jan. 1924; BMNH • 1 क; same collection data as for preceding but 6-25 Feb. 1924; BMNH. - Gauteng • 1 क; Boukenhoutkloof; 18 Dec. 1910; J.V. Niekerk leg.; TMP・ 2 §ð; Pretoria;

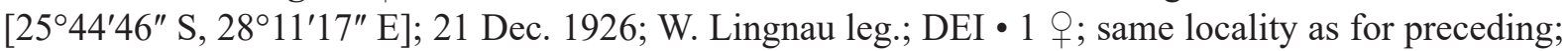

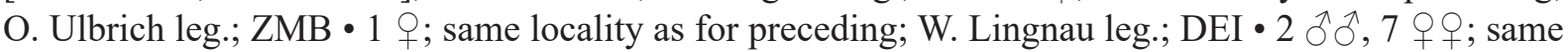

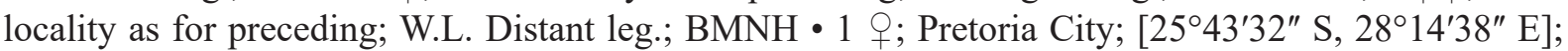
12 Feb. 2000; J. Halada leg.; OÖLM. - KwaZulu-Natal • 1 § ; MNHN • 1 ; ; 10 miles W of Durban; [2950'08.5" S, 3051'2.4" E]; 4 Dec. 1966; J.G. Rozen and D.J. Brothers leg.; AMNH・1 ơ; 20 km NE

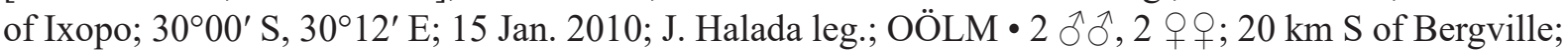

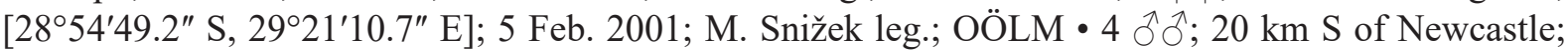

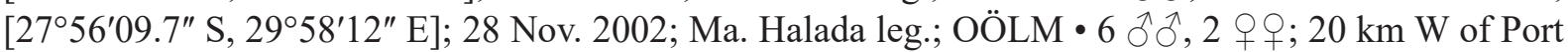

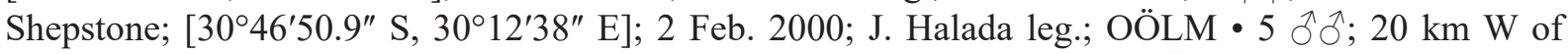

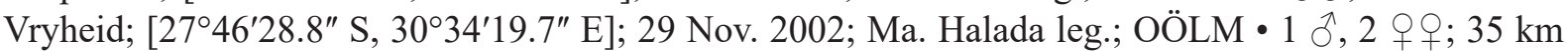
N of Port Edward; 3045' S, 3009' E; 28 Dec. 2009; J. Halada leg.; OÖLM • 1 क; Drakensberge, Royal

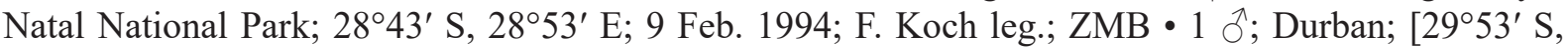

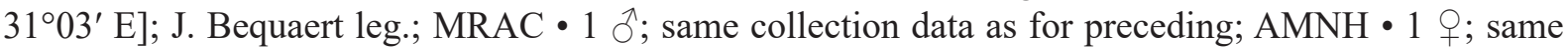
collection data as for preceding; BMNH • 1 O ; Durban, Congella; [29 $\left.53^{\circ} \mathrm{S}, 31^{\circ} 03^{\prime} \mathrm{E}\right]$; 2 May 1908;

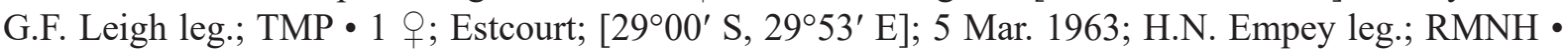

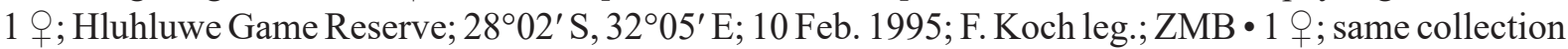
data as for preceding but 5 Dec. 1995; ZMB - 2 ภㅅ, 9 우우; Howick; [ $\left[29^{\circ} 28^{\prime} 40^{\prime \prime} \mathrm{S}, 30^{\circ} 13^{\prime} 50^{\prime \prime} \mathrm{E}\right]$; 1904; J.P. Cregoe leg.; BMNH • 1 ôn, 1 q; Ingogo, N of Newcastle; [2734'40.8" S, 2954'43.2" E]; 28 Nov. 2002; Ma. Halada leg.; OÖLM • 1 o ; Ithala Game Reserve; $27^{\circ} 30^{\prime} \mathrm{S}, 31^{\circ} 20^{\prime} \mathrm{E}$; $28-30$ Jan. 1995; F. Koch leg.; THD-002-ZMB; GenBank CO1 gene: MW538571; GenBank EF-1 $\alpha$ gene: MW558237;

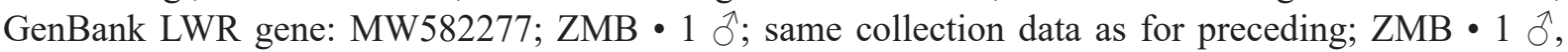

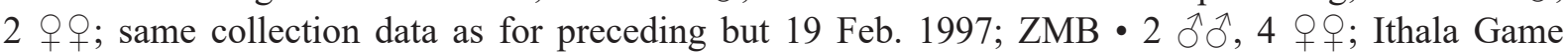

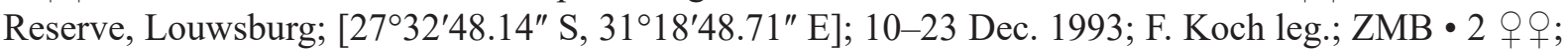
Ithala Game Reserve, sports ground; $27^{\circ} 30^{\prime}$ S, $31^{\circ} 20^{\prime}$ E; 5 Apr. 2001; F. Koch leg.; ZMB • 1 q ; Lake St

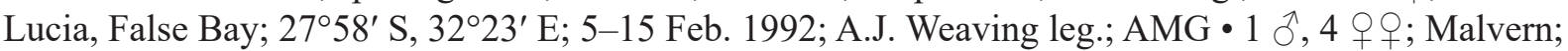

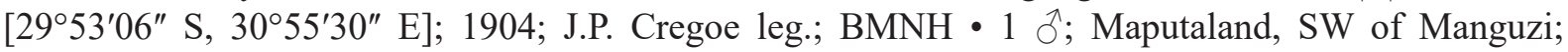

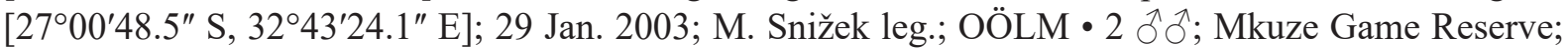
$27^{\circ} 40^{\prime}$ S, 32¹9' E; 22 Nov. 1999; F. Koch leg.; ZMB • 1 q; Ndumo Game Reserve; $26^{\circ} 55^{\prime}$ S, 32 $2^{\circ} 19^{\prime}$ E; 19 Nov. 1999; F. Koch leg.; ZMB • 1 q; Pinetown; [2949'14" S, 3053'12" E]; 18 Mar. 1909; G.F. Leigh

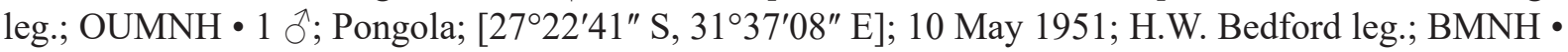




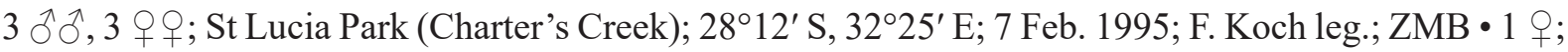

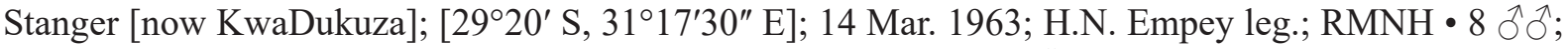
Utrecht; [27³9'36" S, 30¹9'48" E]; 28 Nov. 2002; M. Snižek leg.; OÖLM・ 1 + ; Weenen; [28 50'57" S,

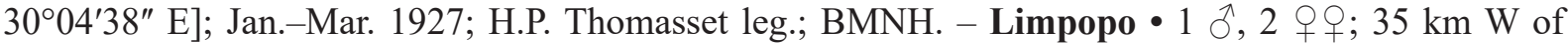

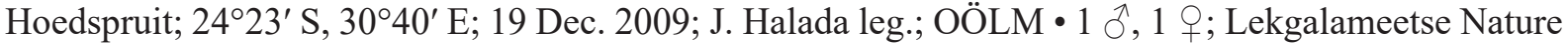

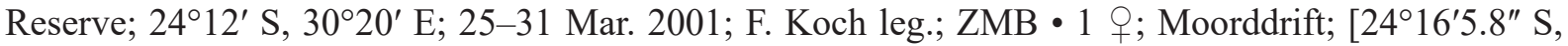

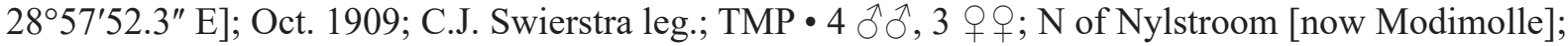

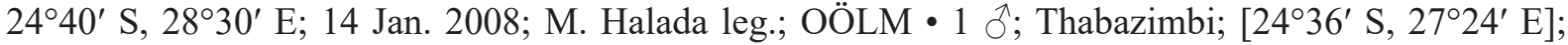
21 Dec. 2008; M. Snižek leg.; OÖLM. - Mpumalanga • 1 §ิ, 1 क; 20 km NW of Nelspruit [now

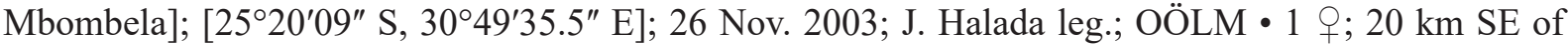

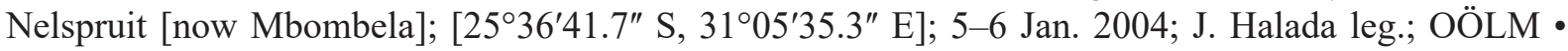

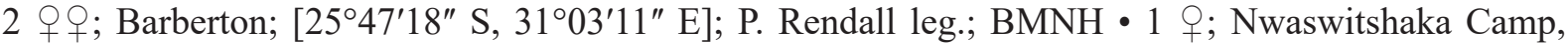

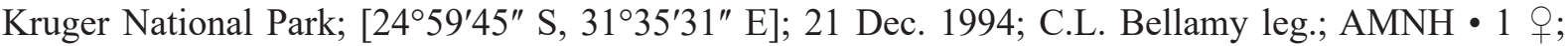
Waterval River; 8 Jan. 1903; J. Breyer leg.; TMP. - North West • 1 ô, 2 q $q$; Rustenburg; [2540'03" S,

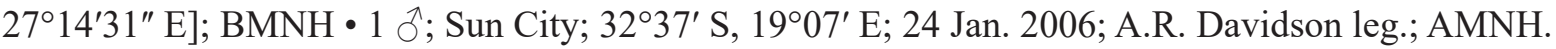

TANZANIA • 1 q; Tabora-Kigoma; Stamper leg.; MRAC. - Dar es Salaam Region • 1 \%; Dar es Salaam; [6 $6^{\circ} 48^{\prime}$ S, 39 $9^{\circ} 17^{\prime}$ E]; 1896; H. Mayer leg.; IRSN. - Katavi Region • 1 ô, 1 \%; $30 \mathrm{~km}$ NNW of Mpanda; 554' S, 3056' E; 30 Dec. 2006; M. Kadlecová leg.; OÖLM. - Kigoma Region • 1 đ̊; 25 km

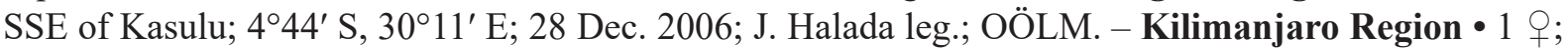
Kilimanjaro, Obstgarten-Steppe; [ $3^{\circ} 05^{\prime} 5.9^{\prime \prime}$ S, 37 20'38.5" E]; 24 Mar. 1905; Y. Sjöstedt leg.; BMNH. -

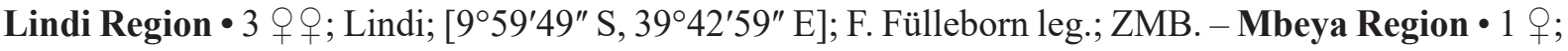
Langenburg [now Tukuyu]; [ $9^{\circ} 15^{\prime} \mathrm{S}, 33^{\circ} 39^{\prime} \mathrm{E}$ ]; end of Feb.-beginning of Mar. 1898; F. Fülleborn leg.;

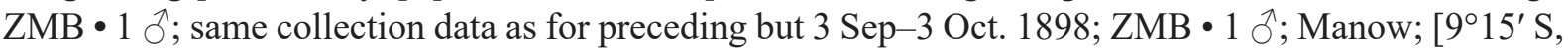

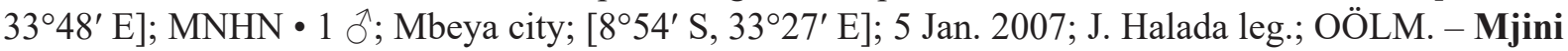

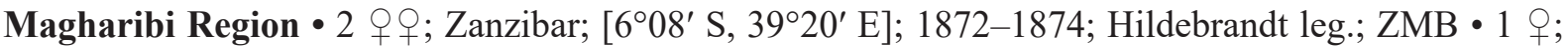
same locality as for preceding; Dec. 1932; A. Seyrig leg.; MNHN. - Rukwa Region • 2 q ; Msamwia, S of Ufipa, S Tanganyika ; [8 ${ }^{\circ} 25^{\prime}$ S, $31^{\circ} 50^{\prime}$ E] ; 10-12 Feb. 1909; Fromm leg.; ZMB. - Ruvuma Region • 1 , lectotype of Sphex nigripes var. pachyderma Strand, 1916 (designated by Oehlke \& Wudowenz 1973:

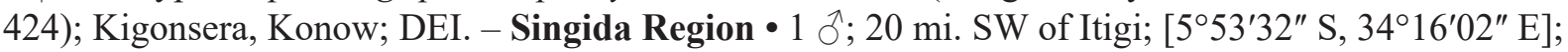
28 Jan. 1970; M.E. Irwin and E.S. Ross leg.; CAS. - Songwe Region • 1 \% ; 70 km NW of Tunduma; $8^{\circ} 58^{\prime}$ S, 32²5' E; 2 Jan. 2007; J. Halada leg.; OÖLM. - Tanga Region • 1 q; "Mnoa”; F. Fischer leg.;

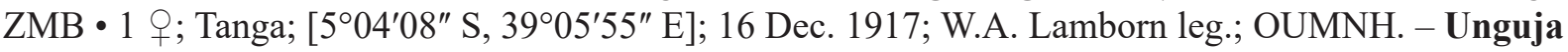

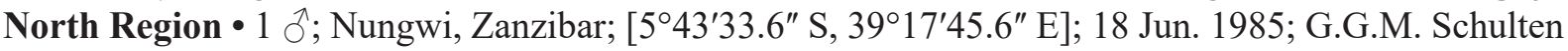
leg.; RMNH.

UGANDA - Eastern Region - 1 ${ }^{\lambda}$; between Jinja and Busia or Mbwago's E Busora (Some Forest); [0 $15^{\circ} 44^{\prime \prime} \mathrm{N}, 33^{\circ} 35^{\prime} 17^{\prime \prime}$ E]; 28 Jul.-1 Aug. 1911; S.A. Neave leg.; BMNH • 1 o'; Mt Kokanjero, SW of Elgon; [107'48" N, 34³3' E]; 7-9 Aug. 1911; S.A. Neave leg.; BMNH.

ZAMBIA • 1 \%; "Mpika - Fort Jameson”; Jan. 1908; S.A. Neave leg.; MRAC • 1 q; Chingola-Solwezi Road; 2 Dec. 2004; M. Snižek leg.; OÖLM. - Central Province • 2 ঐ̊

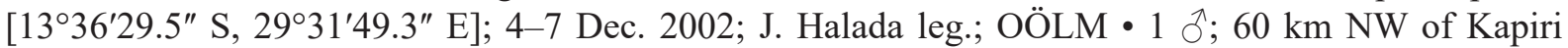

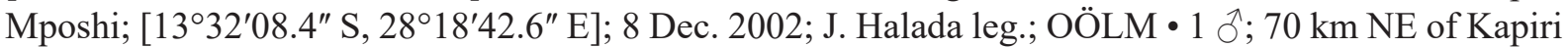
Mposhi; [134ㄴ S, $29^{\circ} 18^{\prime}$ E]; 21 Nov. 2005; M. Snižek leg.; OÖLM • 1 क; Broken Hill [now Kabwe];

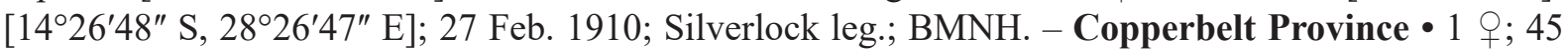

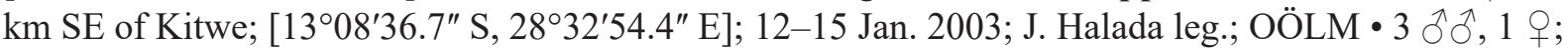
$50 \mathrm{~km} \mathrm{~W}$ of Chingola; [12² $25^{\prime} 06.3^{\prime \prime} \mathrm{S}, 2^{\circ} 23^{\prime} 50.9^{\prime \prime}$ E]; 9-11 Dec. 2002; J. Halada leg.; OÖLM • 2 우; same collection data as for preceding but 1-2 Jan. 2003; OÖLM 11 त ; $80 \mathrm{~km} \mathrm{~W}$ of Chingola;

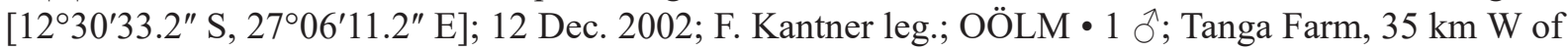


Chingola; [12³2'27.7" S, 27³1'59.4" E]; 6 Nov. 2005; M. Snižek leg.; OÖLM. - Eastern Province •

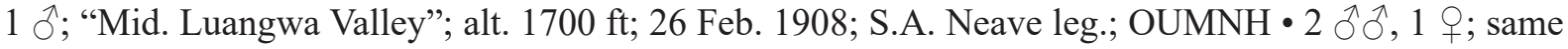
collection data as for preceding but 27 Feb. 1908; OUMNH • 4 우; "On road Ft. Jameson to Lundazi"; 7-14 Jun. 1910; S.A. Neave leg.; BMNH・1 o; "Up. Luangwa Valley”; alt. 1800-2000ft; 30 Mar. 1908; S.A. Neave leg.; OUMNH $\bullet 2$ q $ᄋ$; same collection data as for preceding but 31 Mar. 1908; OUMNH • $1 \delta^{\lambda}$; same collection data as for preceding but 1 Apr. 1908; OUMNH $\bullet 1$ क ; $6 \mathrm{~km}$ E of Sinda; $14^{\circ} 12^{\prime} \mathrm{S}$,

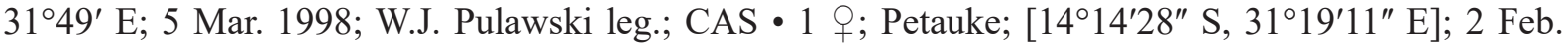
1905; S.A. Neave leg.; OUMNH. - Lusaka Province • 3 우; 25 km E of Lusaka; 15²1' S, 28 $8^{\circ} 30^{\prime}$ E; 3 Mar. 1995; W.J. Pulawski leg.; CAS. - Muchinga Province 2 우; "Mid. Chambezi Valley, Chinsali distr."; alt. $4000 \mathrm{ft}$; 25 Apr. 1908; S.A. Neave leg.; OUMNH • 1 \%; same collection data as for preceding but 27 Apr. 1908; OUMNH 1 \% ; same collection data as for preceding but 29 Apr. 1908; OUMNH • 1 क; Chinsali and neighbourhood; [10³2'29" S, 3204'53" E]; alt. 4300 ft; 9 Apr. 1908; S.A. Neave leg.; OUMNH. - Northern Province • 1 O; "L. Chambezi V.; Kasama distr."; alt. 3900 ft; 10 May 1908; S.A. Neave leg.; OUMNH • 1 q; 5 mi. S of Senga Hill; [9²6'44" S, 31 ${ }^{\circ} 13^{\prime} 21^{\prime \prime}$ E]; 1 Feb. 1970; M.E. Irwin and

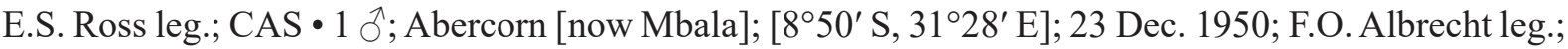
BMNH - 1 त; same collection data as for preceding but 10 Jan. 1951; BMNH. - Southern Province •

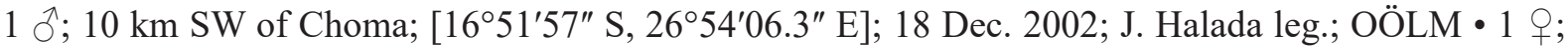

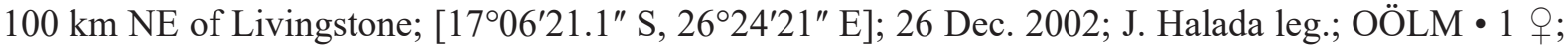

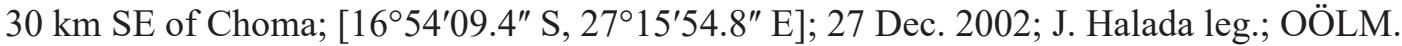

ZIMBABWE • 1 o; "Murntree"; 21 Jan. 1912; G. Arnold leg.; BMNH • 1 q; Victoria Falls; Feb. 1935;

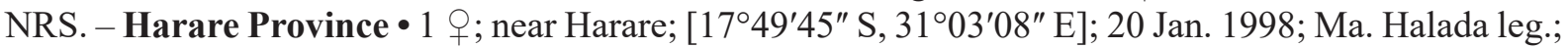

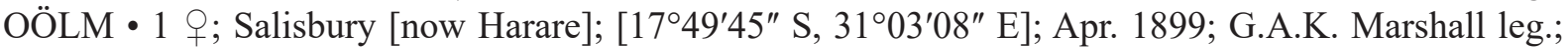
$\mathrm{BMNH} \bullet 2$ $\widehat{\partial}, 1$ q; same collection data as for preceding but Jan. 1900; BMNH • 1 q; same collection data as for preceding but 13 Dec. 1902; OUMNH -3 q 9 ; same locality as for preceding; A. Watsham leg.; BMNH. - Mashonaland Central • 1 o; 10 mi. E of Pakasa [Bakasa]; [16 25'39" S, 3050'32" E]; 28

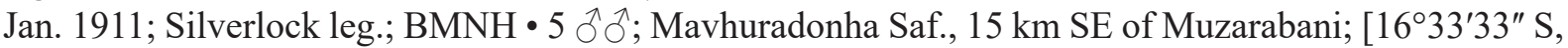
31 ${ }^{\circ} 14^{\prime} 22.8^{\prime \prime}$ E]; 17 Dec. 1998; J. Halada leg.; OÖLM. - Mashonaland East • 2 q $ᄋ$; Chishawasha, near

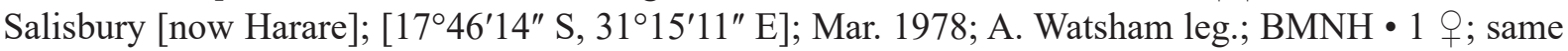
collection data as for preceding but Dec. 1978; BMNH 1 1 9 ; same collection data as for preceding but Feb. 1979; BMNH • 1 ; ; same collection data as for preceding but Dec. 1981; BMNH. - Mashonaland

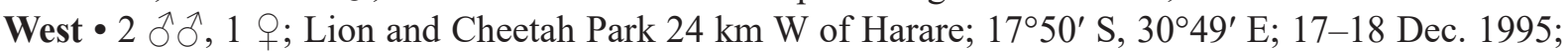
W.J. Pulawski leg.; CAS. - Midlands • 1 q; Selukwe [now Shurugwi]; [1940' S, 3000' E]; 1911; A. Ellenberger leg.; MNHN.

\section{Description}

Female

Size. 22.1-25.8 mm.

COLOR. Black except for basal half of mandible, which is ferruginous. Wings fuscous, with cyan-violet iridescence.

VeSTITURE. Appressed setae on clypeus black, on paraocular area silvery with brown luster, on collar, scutum and propodeal enclosure black. Erect setae on clypeus, paraocular area, collar, scutum and propodeal enclosure black. Erect propodeal setae oriented perpendicularly, slightly curved towards anterior. Central and lower part of clypeus glabrous. Scutellum densely and coarsely pubescent.

STRUCTURE. Free clypeal margin medially with broad, notched tooth, stepped above. Clypeus without indentation or carina. Scutellum convex. Metanotum slightly raised, not notably bituberculate. $2^{\text {nd }}$ recurrent vein joins at interstitium between submarginal cells II and III. Propodeal enclosure without 

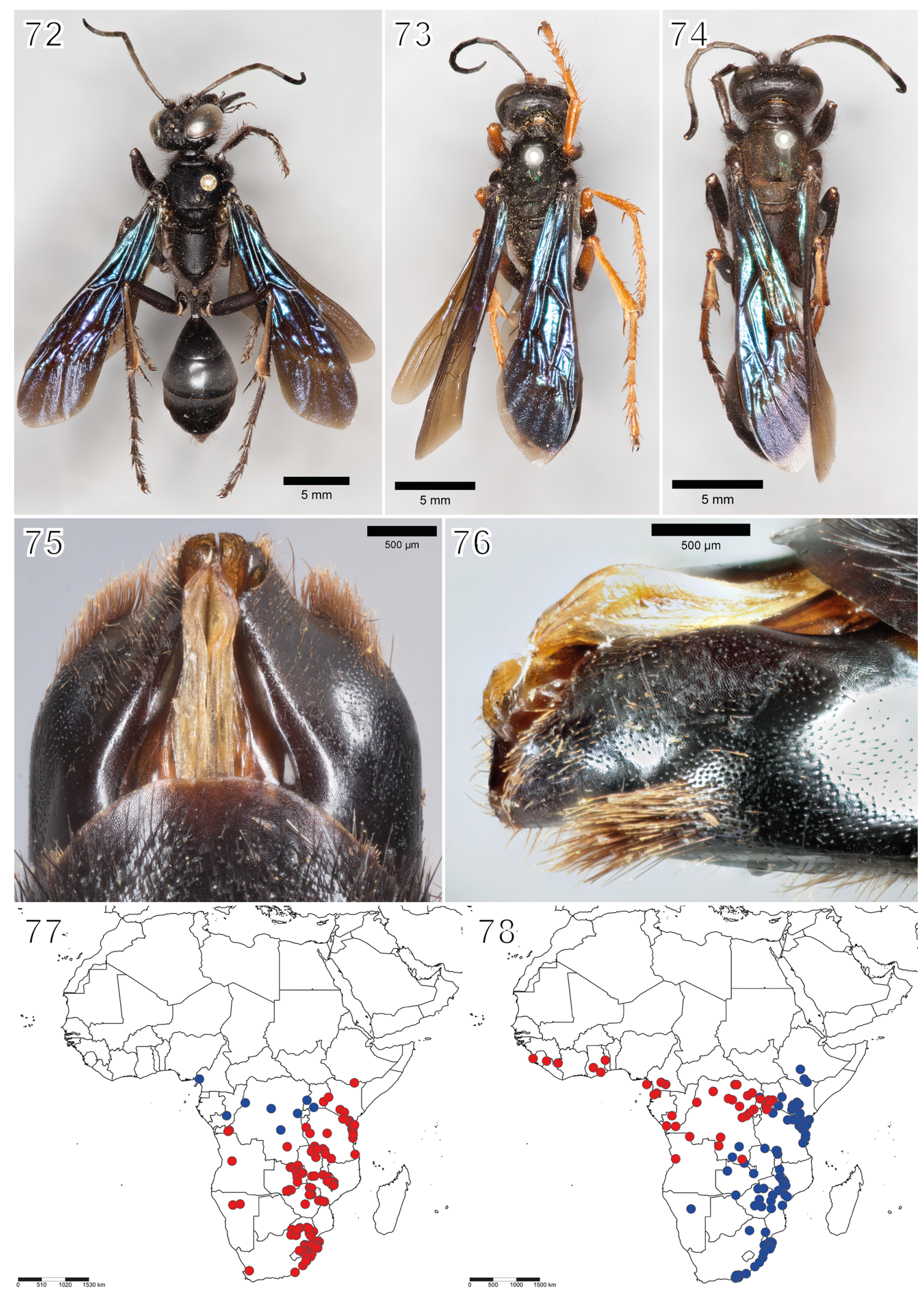

Figs 72-78. 72. Sphex umtalicus Strand, 1916. 73-74. S. victoria sp. nov. 75-76. S. ahasverus Kohl, 1890, male genitalia. 72-73. Habitus of 9.74 . Habitus of ${ }^{7}$. 75. Dorsal view. 76. Lateral view. 77. Geographic distribution of S. umtalicus (red) and S. ahasverus (blue). 78. Geographic distribution of S. camerunicus Strand, 1916 (red) and S. nigrohirtus Kohl, 1895 (blue). 
any notable ridges. Foretarsomere I 2.7-3.6 $\times$ length of antepenultimate spine. Petiole length 1.9-2.5× its medial width.

\section{Male}

SizE. $19.6-24.3 \mathrm{~mm}$.

Color. Black, except for ferruginous stripe in center of mandible. Wings fuscous, with cyan-violet iridescence.

VESTITURE. Appressed setae on clypeus and paraocular area silvery, on collar, scutum and propodeal enclosure black. Erect setae on clypeus, paraocular area, collar, scutum and propodeal enclosure black. Erect propodeal setae oriented perpendicularly, slightly curved towards anterior. Lower center of clypeus glabrous. Scutellum densely and coarsely pubescent.

STRUCTURE. Free clypeal margin medially with tooth emerging from inner side. Scutellum convex. Metanotum slightly raised, not notably bituberculate. $2^{\text {nd }}$ recurrent vein joins interstitium between submarginal cells II and III or slightly proximally. Propodeal enclosure without any notable ridges. Posterior margin of metasomal tergum VII convex. Posterior margin of metasomal sternum VII simple, of metasomal sternum VIII triangular. Petiole length 2.0-2.8 $\times$ its medial width. Flagellomere V and often VI with moderately broad placoid covering their entire length and tapering toward base and apex.

\section{Variation}

Specimens from Lesotho and Gauteng Province (var. basuto/kobrowi) can vary as follows:

\section{Female}

COLOR. Black except for the following, which are ferruginous: basal half of mandible, lower part of clypeus, scape, pedicel, fore and midlegs from apical half of femur, hindleg from femur onward, excluding apical half of claw, and apical metasomal segment. Wings fuscous, with violet luster.

VESTITURE. Appressed setae on clypeus brassy-ferruginous, on paraocular area silvery, on collar, scutum and propodeal enclosure black. Erect setae on clypeus ferruginous near free margin, black otherwise, on paraocular area, collar, scutum and propodeal enclosure black. Erect propodeal setae oriented perpendicularly, slightly curved towards anterior. Lower center of clypeus glabrous. Scutellum densely and coarsely pubescent.

STRUCTURE. Free clypeal margin medially with broad, notched tooth, stepped above. Clypeus with indistinct indentation in lower center, without carina. Scutellum convex. Metanotum raised, not notably bituberculate. $2^{\text {nd }}$ recurrent vein joins slightly proximal from interstitium between submarginal cells II and III. Propodeal enclosure without any notable ridges. Foretarsomere I 2.8-2.9 $\times$ length of antepenultimate spine. Petiole length $1.70-1.83 \times$ its medial width.

\section{Male}

COLOR. Black except for the following, which are ferruginous: basal half of mandible, femora apically, tibiae, and mid- and hindtarsomeres I-III. Wings hyaline, with yellowish tinge, apical margin of forewing infuscate.

VeSTITURE. Appressed setae on clypeus and paraocular area silvery, on collar and scutum black, on propodeal enclosure brownish-gray. Erect setae on clypeus, paraocular area, collar, scutum and propodeal enclosure black. Erect propodeal setae oriented perpendicularly, slightly curved towards anterior. Lower center of clypeus glabrous. Scutellum densely and coarsely pubescent. 
StruCture. Free clypeal margin medially with tooth emerging from inner side. Scutellum convex. Metanotum slightly raised, not notably bituberculate. $2^{\text {nd }}$ recurrent vein nearly joins interstitium between submarginal cells II and III. Propodeal enclosure without any notable ridges. Posterior margin of metasomal tergum VII convex. Posterior margin of metasomal sternum VII simple, of metasomal sternum VIII triangular. Petiole length $1.5-1.6 \times$ its medial width. Flagellomere V with moderately broad placoid covering its proximal four-fifths.

\section{Distribution}

Eastern, southeastern and southwestern Africa.

\section{Remarks}

As van der Vecht (1961) correctly stated, the name Sphex haemorrhoidalis Fabricius, 1781, which Arnold (1928) used for black-legged specimens, is in fact linked to a red-legged taxon from western Africa. Other authors like Leclercq $(1955,1961)$, Berland (1956) and Dollfuss (2008) have based their identifications on Arnold's mistake, and therefore many locality records and previous synonymizations were erroneous.

Although we were unable to examine the types of $S$. kobrowi (despite our request, it was apparently not included in the loan from TMP) or S. basuto, we nonetheless feel that our opinion on their synonymy is justified. First and foremost, we did study specimens which matched the descriptions and were most likely from the same collecting series as the types of both taxa, as they shared their locality, time frame and presumed collector. Labels indicated that Arnold had examined some of them as well. Secondly, we would have proposed to synonymize $S$. basuto with $S$. kobrowi even without additional evidence beyond their original descriptions. Judging from Arnold's diagnosis, both resemble each other very much and are only distinguished through $S$. basuto having "thinner" propodeal pubescence and a slightly different interocular distance as well as flagellomere length. The measurements for the varieties of $S$. haemorrhoidalis in Arnold (1947) all seem quite dubious, especially in the case of $S$. basuto and $S$. kobrowi, with differences in length of less than $4 \%$ being used to differentiate between them. In addition, the actual numbers given are virtually useless for identifications without material for comparison, as they are highly dependent on the angle from which the measurements are taken and the how the boundaries between areas are perceived, yet these details are nowhere mentioned.

Though distinct in coloration and with a slightly different ratio of petiole length to width, S. kobrowi appears to be genetically identical to $S$. umtalicus. A comparison of parts of the CO1 sequences of specimens conforming to both taxa showed their complete congruence.

Sphex victoria sp. nov. urn:lsid:zoobank.org:act:DDA85F4D-70BE-49E5-BA6E-5F9A97D64760

Figs 71 (blue), 73-74

\section{Differential diagnosis}

Females of S. haemorrhoidalis (Fig. 69) and S. victoria sp. nov. (Fig. 73) cannot always be distinguished satisfactorily. Especially in specimens from central Africa, where their populations overlap, it is often difficult to decide what color the luster of the appressed paraocular and clypeal setae is, which is one of the only useful diagnostics. The coloration of the legs can also be somewhat helpful, but is variable as well. Thus, the character states mentioned represent the more distinct manifestations that occur mostly in eastern and western Africa for S. victoria sp. nov. and S. haemorrhoidalis, respectively.

In the female of $S$. haemorrhoidalis, the appressed setae laterally on the clypeus and on the paraocular area have a ferruginous luster (Fig. 34). In contrast, it has a silvery luster in S. victoria sp. nov. 
(Fig. 35). Furthermore, S. victoria sp. nov. has at least the apical half of the hindfemora ferruginous, whereas in S. haemorrhoidalis the hindfemora are predominantly black. Females of S. mweruensis are distinguishable through having the erect setae at the posterior propodeal margin silvery (Fig. 64), while those of S. umtalicus have the clypeus (Fig. 33) and most of the body black (Fig. 72), and both sexes of S. caeruleanus can be recognized though their very distinctly green wing iridescence (Fig. 63).

Males (Fig. 74) are identifiable through the combination of having uniformly black setae on the thoracical dorsum, the mandibular base brightly ferruginous and the hindtibia at least partially ferruginous. Those of S. haemorrhoidalis (Fig. 70) and S. mweruensis (Fig. 65) have a substantial number of erect mesanotal setae silvery, and $S$. umtalicus has the legs uniformly black.

\section{Etymology}

This species was named after Lake Victoria, as most of the material was collected in its vicinity.

\section{Material examined}

Holotype

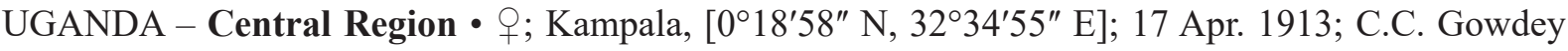
leg.; BMNH.

\section{Paratypes}

AFRICA • 1 9; "British East Africa"; 1906; Maurice de Rothschild leg.; MNHN.

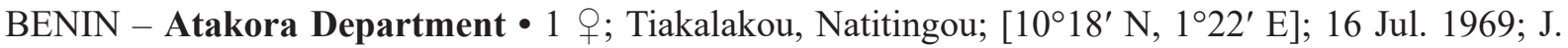
Hamon leg.; MNHN.

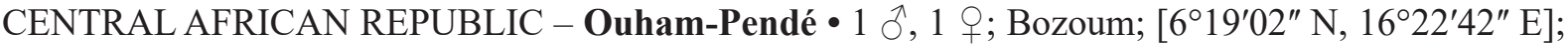
1-10 Jun. 1914; G. Tessmann leg.; ZMB 1 1 ; same collection data as for preceding but 11-20 Jun. 1914; ZMB.

DEMOCRATIC REPUBLIC OF THE CONGO - Haut-Katanga • 1 q; "Basse Pande"; 15 Apr. 1944;

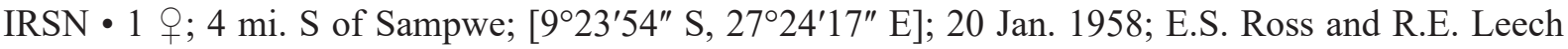

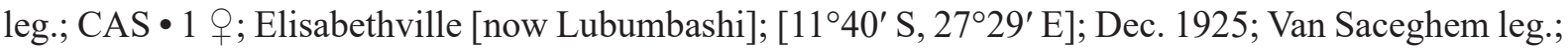

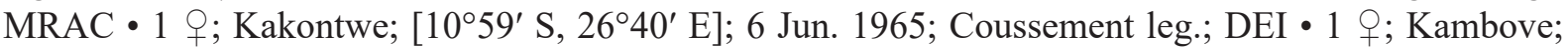

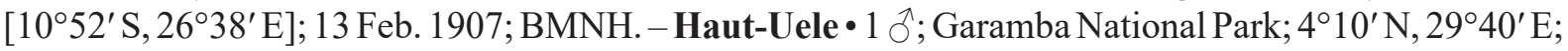
Jun.-Jul. 1912; Lang and Chapin leg.; AMNH $\bullet 1$ $\delta, 4$ + $\odot$; same collection data as for preceding but Jul. 1912; AMNH. - Kongo Central • 1 क ; Boma; $6^{\circ} 00^{\prime}$ S, $13^{\circ} 00^{\prime}$ E; 17 Jun. 1915; Lang and Chapin leg.;

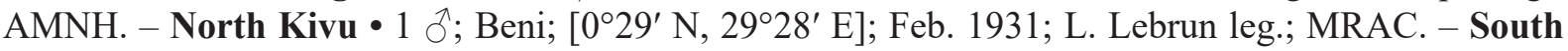
Kivu • 1 क; Kadjudju; [209’ S, 2854' E]; 1931; G. Babault leg.; MNHN.

KENYA • 3 + $\odot$; Kakumga [Kakamega] Forest; [0¹7'30" N, 3451'22" E]; 21-28 May 1911; S.A. Neave leg.; MNHN • 1 क; Kisima to Mumias Road; Aug. 1912; A.D. Milne leg.; MNHN • 1 q; Mogorr River; May 1913; A.O. Luckman leg.; BMNH • 2 o ○; Upper Kuja Valley; S of Kavirondo; alt. 4200 ft; 5-8 May 1911; S.A. Neave leg.; BMNH $\bullet 8 \hat{\jmath} \hat{\jmath}, 13$ q ${ }_{+}$; Yala River, S edge of Kakumga [Kakamega] Forest; 21-28 May 1911; S.A. Neave leg.; BMNH. - Bungoma County • 3 우; S foot and slopes of Mt Elgon; [047'46" N, 34³5'57" E]; 8-13 Jun. 1911; S.A. Neave leg.; BMNH. - Kakamega County •

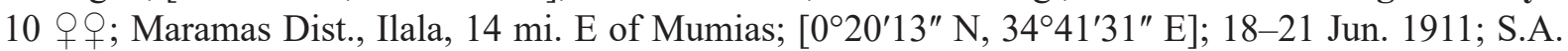

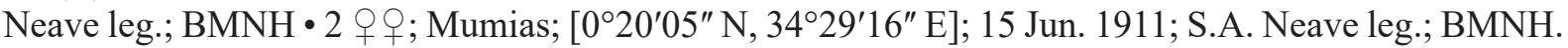
- Kisii County • 1 क ; Kisii District, S Kavirondo; [040’ S, 3445’ E]; 9-12 May 1911; S.A. Neave leg.; BMNH. 


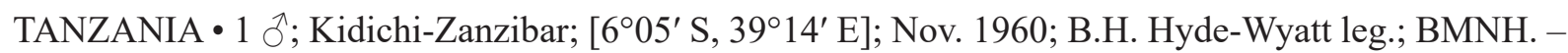

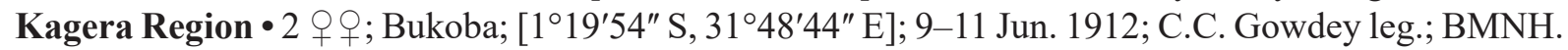

UGANDA • 1 đ’; Banks of Nile near Kakindu; alt. 3400 ft; 24-25 Aug. 1911; S.A. Neave leg.; BMNH •

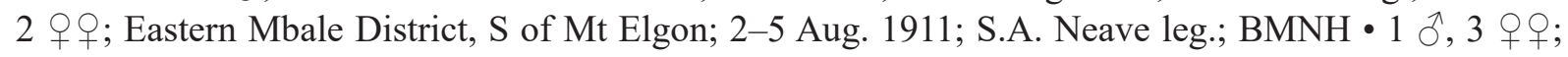
Milgana; 30 Apr. 1911; C.C. Gowdey leg.; BMNH. - Central Region • 1 q; 3-4 mi. NE of Entebbe;

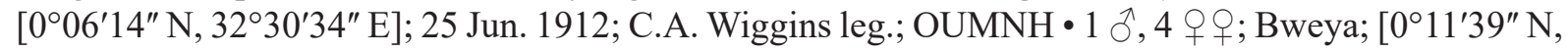
32³2'59" E]; 10 May 1913; C.C. Gowdey leg.; BMNH・3 우; same collection data as for preceding but 17 May 1913; BMNH -2 q $q$; same collection data as for preceding but 22 May 1913; BMNH •

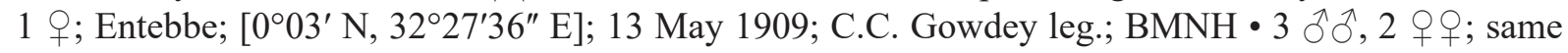

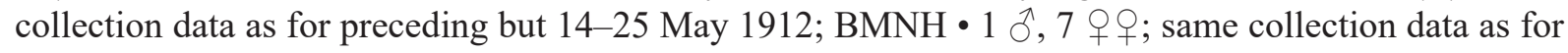
preceding but 30-31 May 1912; BMNH 1 1 ; same collection data as for preceding but 15-22 Jun. 1912; BMNH • 2 ㅇ; ; same collection data as for preceding but Aug. 1912; BMNH • 3 q $ᄋ$; same collection data as for preceding but 24-25 May 1913; BMNH • 2 q 9 ; same collection data as for preceding but 30 May 1913; BMNH 1 \%; same collection data as for preceding but 12 Jun. 1913; $\mathrm{BMNH} \bullet 1$ + ; same collection data as for preceding but 17 Jun. 1913; BMNH 1 1 ; ; same locality as for preceding; 29 May 1909; C.A. Wiggins leg.; OUMNH 1 § ; same collection data as for preceding but 5 Jul.-7 Aug. 1909; OUMNH 11 ; ; same collection data as for preceding but 6-13 May 1912; BMNH • 1 $\circ$; same locality as for preceding; May 1906; Maurice de Rothschild leg.; MNHN • 4 우; same collection data as for holotype; BMNH • 3 q o ; Mabira Forest; [0²9'03" N, 32 59'05" E]; 2 Jul. 1913; C.C. Gowdey leg.; BMNH • 1 क; Mabira Forest, Chagwe; [0²9'03" N, 3259'05" E]; 16-25 Jul. 1911;

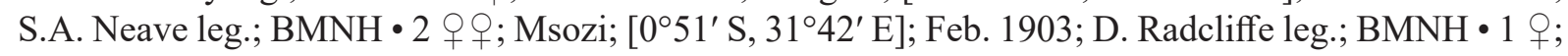
Mwera; [0²7'47" N, 32॰19'56" E]; 2 Aug. 1913; C.C. Gowdey leg.; BMNH. - Eastern Region • 4 q $O$;

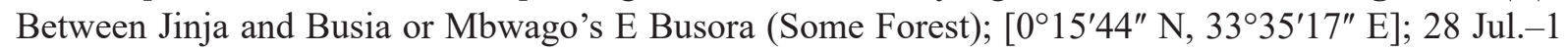
Aug. 1911; S.A. Neave leg.; BMNH • 1 क; Between Kumi and NE shore of Lake Kioga; [1 ${ }^{\circ} 32^{\prime} 09^{\prime \prime}$ N,

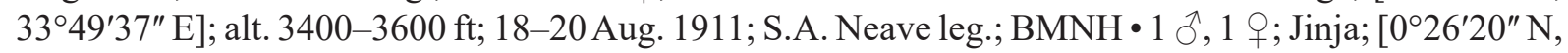

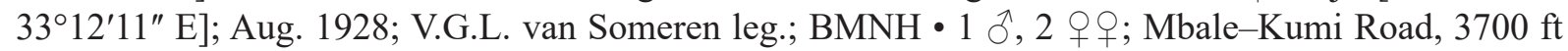

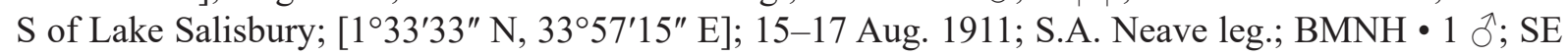

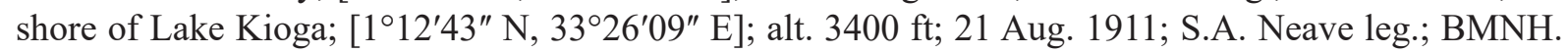
- Western Region • 1 \%; Fort Portal Road, Mbarara, Southern Toro; [0³6'48" S, 30³8'54" E]; 22-24 Oct. 1911; S.A. Neave leg.; BMNH • 4 ठิð, 2 우; SE of Ankole; 4-8 Oct. 1911; S.A. Neave leg.; BMNH.

\section{Description}

\section{Female}

Size. $23.0-25.0 \mathrm{~mm}$.

CoLOR. Black except for the following, which are ferruginous: basal half of mandible, clypeus, scape, pedicel and legs from apical half of femur onward, excluding apical half of claw. Wings fuscous, with cyan-violet iridescence.

VeSTITURE. Appressed setae on clypeus and paraocular area silvery, on collar, scutum and propodeal enclosure black. Erect setae on clypeus ferruginous, black towards dorsal margin, on paraocular area, collar, scutum and propodeal enclosure black. Erect propodeal setae oriented perpendicularly, slightly curved towards anterior. Lower center of clypeus glabrous. Scutellum densely and coarsely pubescent.

STRUCTURE. Free clypeal margin medially with broad, notched tooth, stepped above. Clypeus without indentation or carina. Scutellum convex. Metanotum slightly raised, not notably bituberculate. $2^{\text {nd }}$ recurrent vein nearly joins interstitium between submarginal cells II and III. Propodeal enclosure without any notable ridges. Foretarsomere I 2.7-3.2 $\times$ length of antepenultimate spine. Petiole length 1.6-2.2 $\times$ its medial width. 


\section{Male}

SizE. 23.2-23.7 mm.

CoLOR. Black except for the following, which are ferruginous: basal half of mandible and hindleg from tibia onward, excepting apical half of claw. Wings fuscous, with cyan-violet iridescence.

VeSTITURE. Appressed setae on clypeus and paraocular area silvery, on collar, scutum and propodeal enclosure black. Erect setae on clypeus, paraocular area, collar, scutum and propodeal enclosure black. Erect propodeal setae oriented perpendicularly, slightly curved towards anterior. Lower center of clypeus glabrous. Scutellum densely and coarsely pubescent.

StRUCTURE. Free clypeal margin medially concave, with tooth emerging from inner side. Scutellum convex. Metanotum slightly raised, not notably bituberculate. $2^{\text {nd }}$ recurrent vein nearly joins interstitium between submarginal cells II and III. Propodeal enclosure without any notable ridges. Posterior margin of metasomal tergum VII convex. Posterior margin of metasomal sternum VII simple, of metasomal sternum VIII triangular. Penis valvae without conspicuous modifications. Petiole length $2.1-2.4 \times$ its medial width. Flagellomeres IV-VI with broad placoids covering their entire length.

\section{Variation}

Unknown.

\section{Distribution}

Eastern to central Africa.

\section{Species of the nigrohirtus group}

The nigrohirtus group contains the following five species: Sphex ahasverus, S. camerunicus, S. incomptus, S. nigrohirtus and S. pulawskii sp. nov.

Members are characterized through posteriorly-oriented erect propodeal setae, which they share with the species of the bohemanni group (Fig. 1). Distinguishing features between both are covered in the introduction to that group.

Sphex ahasverus Kohl, 1890

Figs 75-76, 77 (blue)

Sphex ahasverus Kohl, 1890: 397,, .

Sphex observabilis R. Turner, 1918: 360, 9 . Syn. nov.

\section{Differential diagnosis}

Males and females of this species are unmistakable within their species group, as they have uniformly golden vestiture covering the pleurae and propodeum, which completely obscures the propodeal sculpture.

\section{Material examined}

Holotype or syntype

AFRICA • +; "South Australia", but actually Africa; NHMW.

Other material

CAMEROON - Southwest Region - 1 \%; "Johann-Albrechts-Höhe" [Government Station Johann Albrecht Mountain]; [440' N, 9²4' E]; 14 May 1896; L. Conradt leg.; ZMB • 1 क; Barombi Station; [4 $40^{\prime} \mathrm{N}, 9^{\circ} 24^{\prime}$ E]; P. Preuss leg.; ZMB. 
DEMOCRATIC REPUBLIC OF THE CONGO • 1 q; "Kasaï”; May 1922; Van Risseghem leg.;

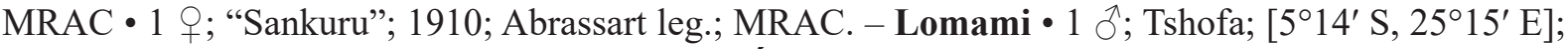

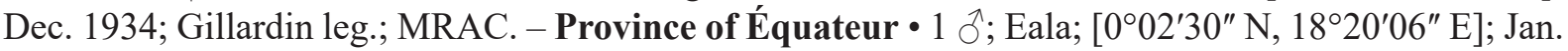
1936; J. Ghesquière leg.; MRAC • 1 సं; same collection data as for preceding but Aug. 1936; MRAC.

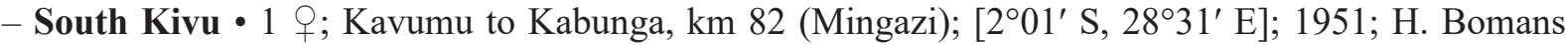

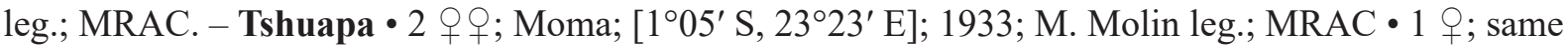
locality as for preceding; Jun. 1925; J. Ghesquière leg.; MRAC.

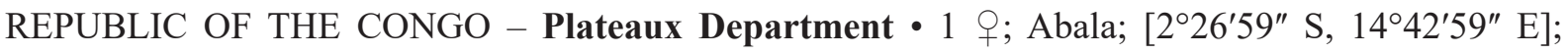
13 Sep. 1951; MNHN.

UGANDA - Central Region 1 1 , holotype or syntype of Sphex observabilis R. Turner, 1918; Tero Forest; [0 $0^{\circ} 50^{\prime} \mathrm{S}, 31^{\circ} 40^{\prime}$ E]; 26-30 Sep. 1911; S.A. Neave leg.; BMNH. - Western Region - 1 万'; Kabarole Kibale Forest National Park, Kanyawara (MUBFS); 0³3' N, 30²1' E; 23 Aug. 1997; C. Häuser leg.; THD-011-ZMB; GenBank CO1 gene: MW538553; GenBank LWR gene: MW582291; $\mathrm{ZMB} \cdot 1$; ; same collection data as for preceding; ZMB.

\section{Description}

Female

Size. $29.4-32.8 \mathrm{~mm}$.

VeSTITURE AND STRUCTURE. A redescription based on the holotype has already been published (Dörfel \& Ohl 2015). Some additional important characters of the species are treated here. Erect propodeal setae oriented posteriorly. Free clypeal margin slightly stepped medially, clypeus with slight indentation in lower center, without carina. $2^{\text {nd }}$ recurrent vein joins markedly proximal from interstitium between submarginal cells II and III. Foretarsomere I 2.6-2.9 $\times$ length of antepenultimate spine. Petiole length $1.4-1.6 \times$ its medial width.

\section{Male}

SIZE. 27.0-29.1 mm.

CoLOR. Black except for ferruginous stripe in center of mandible. Forewing slightly fuscous, hindwing mostly hyaline.

VestiTuRe. Appressed setae on clypeus, paraocular area, collar and propodeal enclosure silverygolden, on scutum black. Erect setae on clypeus and paraocular area black intermixed with silvery ones, on scutum black, on collar and propodeal enclosure silvery-golden. Erect propodeal setae oriented posteriorly. Free clypeal margin glabrous. Scutellum densely and coarsely pubescent. Metasomal sterna II-V with decreasingly dense, VI-VII with increasingly dense fringes of black setae.

STRUCTURE. Free clypeal margin simple. Scutellum convex. Metanotum slightly raised, not bituberculate. $2^{\text {nd }}$ recurrent vein joins markedly proximal from interstitium between submarginal cells II and III. Propodeal enclosure without any notable ridges. Posterior margin of metasomal tergum VII very gently notched. Posterior margin of metasomal sternum VII simple, of metasomal sternum VIII slightly concavely emarginate. Penis valvae fused, inner margin flattened and conspicuously raised, dorsally broadened. Petiole length $1.35-1.45 \times$ its medial width. Flagellomeres IV-VII with broad placoids covering their entire length.

\section{Variation}

Unknown. 


\section{Distribution}

Central Africa.

\section{Remarks}

Members of Sphex observabilis are indistinguishable from the type of $S$. ahasverus, allegedly from South Australia, which leads us to the conclusion that both species are synonymous. However, we do not believe that there are morphologically identical populations of the species in both Uganda and South Australia. The specimens Kohl (1890) described as S. ahasverus were apparently part of a batch which the Vienna Natural History Museum had received in 1804 from Leopold von Fichtel. A locality error is therefore conceivable, since a large part of the material came from Africa (Rogenhofer 1889). Even though R. Turner treated S. ahasverus in his key to the Australian Sphecini (1910) and described S. observabilis in 1918, he likely failed to notice the connection between the two. Thus, observabilis should become an invalid name, while ahasverus is to be used for the species even though it is most likely restricted to Africa in its geographical distribution.

\section{Sphex camerunicus Strand, 1916}

Figs 78 (red), 79-80, 83

Sphex bohemanni var. camerunicus Strand, 1916: 105, ㅇ. Sphex conradti Berland, 1927: 145, + . Syn. nov.

\section{Differential diagnosis}

Females can be recognized through a combination of having basally black mandibles, completely black erect propodeal setae and appressed silvery setae on the collar, as well as posterolaterally on the scutum (Fig. 83). In contrast, those of S. incomptus, S. nigrohirtus and S. pulawskii sp. nov. usually have the basal mandible half and the free clypeal margin medially ferruginous. Female $S$. nigrohirtus have uniformly black setae on the collar, scutum and propodeal enclosure (Fig. 84), whereas those of S. incomptus also have the majority of the propodeal setae silvery, in addition to the silvery appressed setae on the collar and the spot posterolaterally on the scutum (Fig. 85). Females of S. pulawskii sp. nov. resemble those of $S$. camerunicus in the color of the setae on the collar, but they are distinguished through their silvery appressed and erect setae at the posterior propodeal margin (Fig. 86). Female S. camerunicus are also considerably larger and have a deeper indentation on the clypeus.

Males of S. camerunicus differ in having broad placoids that completely cover flagellomeres IV-VII, and their clypeal indentation and carina are more pronounced as well. Excepting the unmistakable S. ahasverus, the other group members only have a narrow, often discontinuous placoid on flagellomere V. Furthermore, the valvicepes of $S$. camerunicus have a concave emargination dorsolaterally (Figs 7980), while this structure is absent in S. nigrohirtus (Figs 81-82), S. incomptus and S. pulawskii sp. nov.

\section{Material examined}

\section{Holotype}

CAMEROON • + ; L. Conradt leg.; DEI.

\section{Other material}

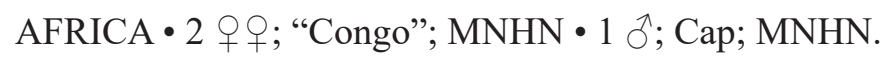

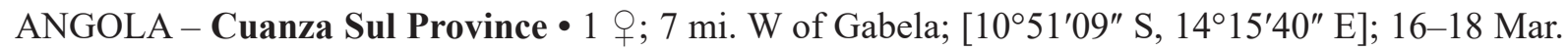
1972; BMNH. 


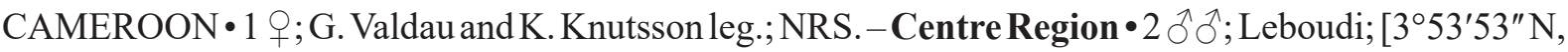

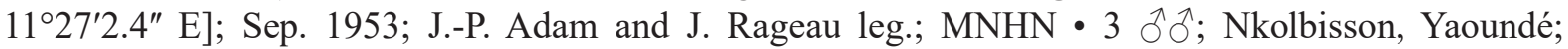

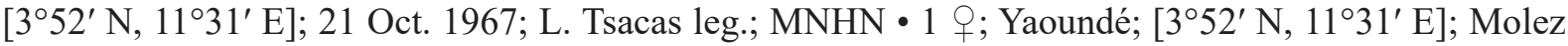
leg.; MNHN • $2 \delta^{\lambda}$; same collection data as for preceding; MNHN. - South Region • 1 q; Douma District; [ $3^{\circ} 30^{\prime} \mathrm{N}, 12^{\circ} 21^{\prime} \mathrm{E}$ ]; Schipper leg.; ZMB.

CENTRAL AFRICAN REPUBLIC • 1 \%; 27 Apr. 1918; M. Boulard leg.; MNHN.

DEMOCRATIC REPUBLIC OF THE CONGO • 1 q; "Kwawa (Bangala)"; 4 Nov. 1922; A. Collart leg.; MRAC • 1 q; "Kwilu"; Dec. 1913-Jan. 1914; P. Vanderijst leg.; MRAC. - Bas-Uele • 1 q; Bambesa;

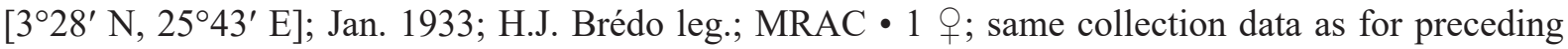
but 25 Sep. 1933; MRAC -5 우; same collection data as for preceding but 20 Oct. 1933; MRAC • 3 + 9 ; same collection data as for preceding but 30 Oct. 1933; MRAC $\bullet 1$ q; same collection data as for

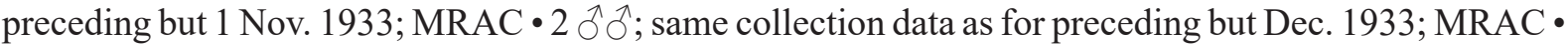
1 क; same locality as for preceding; 14 Aug. 1937; J. Vrijdagh leg.; MRAC 1 ô; same collection data as for preceding but 17 Mar. 1939; IRSN • 1 क; same locality as for preceding; 9 May 1938; P. Henrard leg.; MRAC • 1 ते; same locality as for preceding; Dec. 1934; J.V. Leroy leg.; MRAC • 1 क ; Dingila; [ $3^{\circ} 27^{\prime}$ N, 260ㅜ' E]; May 1933; H.J. Brédo leg.; MRAC • 1 q; Poko-Nala-Rungu; Jan. 1912;

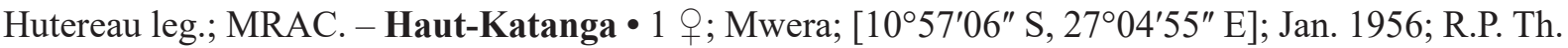
de Caters leg.; MRAC. - Ituri Province • 1 o; Nduye-Makara; [1 ${ }^{\circ} 51^{\prime} 33^{\prime \prime}$ N, $2^{\circ} 00^{\prime} 57^{\prime \prime}$ E]; Sep.-Oct. 1921; A. Pilette leg.; MRAC $11^{\top}$; Penge; [ $1^{\circ} 20^{\prime}$ N, $28^{\circ} 09^{\prime}$ E]; 21 Feb. 1919; J. Bequaert leg.; MRAC. - Kasaï-Central • 1 q; Kamponde; [6 $6^{\circ} 41^{\prime} 53^{\prime \prime}$ S, 22 $2^{\circ} 55^{\prime} 44^{\prime \prime}$ E]; 1945; MRAC. - Kongo Central •

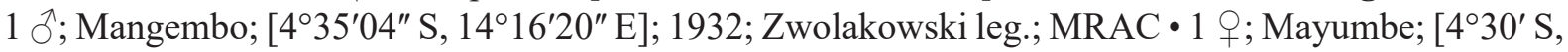
$12^{\circ} 30^{\prime}$ E]; 1917; R. Mayné leg.; MRAC. - Kwango • 1 ô, 1 q; Kingunda; [6³4' S, 16 $6^{\circ} 58^{\prime}$ E]; 17 Jan.

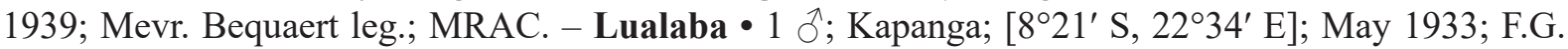
Overlaet leg.; MRAC. - Maniema • 1 ơ; Lubutu; [044' S, 26³5' E]; 22 Jan. 1915; J. Bequaert leg.; MRAC. - Mongala Province • 1 q; Lisala; $\left[2^{\circ} 09^{\prime}\right.$ N, $21^{\circ} 30^{\prime}$ E]; 1940; Leontowitch leg.; MRAC. North Kivu • $3 \widehat{\partial} \widehat{\partial}, 6$ + $\odot$; Parc National de Virungas, Oycha region; Apr.-May 1950; J. de Wilde leg.;

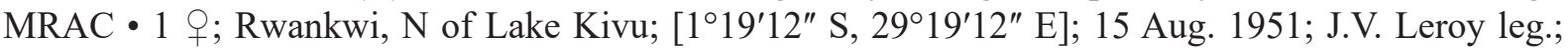

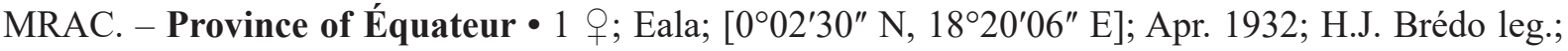
MRAC • 1 \%; same collection data as for preceding but 30 Oct. 1933; MRAC • 2 우; same locality as for preceding; Apr. 1933; A. Corbisier leg.; MRAC • 1 \%; same locality as for preceding; Apr. 1935; J. Ghesquière leg.; MRAC $\bullet 1$; same collection data as for preceding but Oct. 1935; MRAC. - South

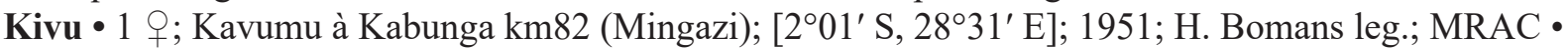

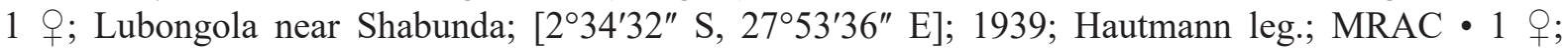
Mulungu; [ $2^{\circ} 53^{\prime}$ S, $27^{\circ} 07^{\prime}$ E]; 26 Sep. 1938; Hendrickx leg.; MRAC. - Tshopo • 1 P; Stanleyville [now

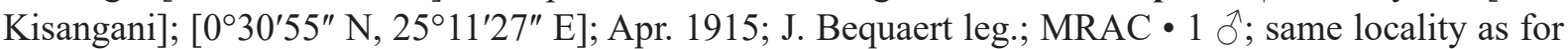
preceding; Jun. 1932; J. Vrydagh leg.; MRAC $\bullet 1$ đ;; same locality as for preceding; 10-13 Sep. 1928; A. Collart leg.; MRAC • 1 o ; Yangambi; [046’ N, 242ㄱ' E]; 1940; MRAC.

EQUATORIAL GUINEA • 1 \%; "Bénito"; MNHN • 1 q, lectotype of Sphex conradti Berland, 1927 (designated by Menke in Bohart \& Menke 1976: 115); Fernando-Po [Bioko]; [3³0' N, 842' E]; 1901; L. Conradt leg.; MNHN 2 우, paralectotypes of Sphex conradti Berland, 1927; same collection data as for lectotype of Sphex conradti Berland, 1927; MNHN. - Centro Sur • 1 q; Monte Alén, Benito River; [1 ${ }^{\circ} 31^{\prime} 48^{\prime \prime}$ N, 1006'36" E]; 16-30 Sep. 1906; G. Tessmann leg.; ZMB • 1 \%; Uelleburg; [1 ${ }^{\circ} 49^{\prime} \mathrm{N}$, $\left.10^{\circ} 36^{\prime} \mathrm{E}\right]$; 15-31 Jan. 1907; G. Tessmann leg.; ZMB • 1 q; same collection data as for preceding but 15-28 Feb. 1907; ZMB • 1 q; same collection data as for preceding but Jun.-Aug. 1908; ZMB • 1 ㅇ; same collection data as for preceding; ZMB.

GHANA - Ashanti Region • 1 q; Tafo; [6 $43^{\prime} 53^{\prime \prime}$ N, 136'49" W]; 26 Oct. 1970; B. Bolton leg.;

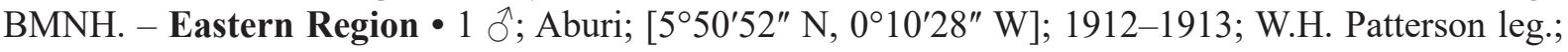
$\mathrm{BMNH} \bullet 1$ क; same locality as for preceding; Dec. 1941; K.M. Guichard leg.; BMNH. 
GUINEA - Nzérékoré Region • 1 क; Mt Nimba; [7³6'09" N, 8²8'5.6" W]; alt. 1000 m; 2 Jun. 1942; M. Lamotte leg.; MNHN • 1 q; Nion, Mt Nimba; [7³6'09" N, 8²8'5.6" W]; 2 Jun. 1942; M. Lamotte leg.; MNHN.

LIBERIA - Lofa County • 1 क; 20 mi. [sic.] Voinjama; 13 Aug. 1966; E.S. Ross and K. Lorenzen leg.; CAS.

REPUBLIC OF THE CONGO - Lékoumou Department • 1 ô; Mbouloupambou; [2³2'26.16" S, 13³2'45.96" E]; 11 Feb. 1980; C. Morin leg.; MNHN.

SIERRA LEONE • 1 क; A. Mocquerys leg.; IRSN • 1 q; W.G. Clements leg.; BMNH. - Eastern Province • 1 O; Bomaru; [800'47" N, 10³7'36" W]; 8 Aug. 1912; J.J. Simpson leg.; BMNH. - Western

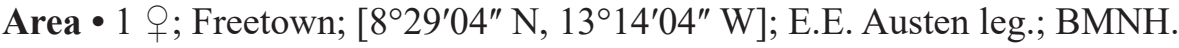

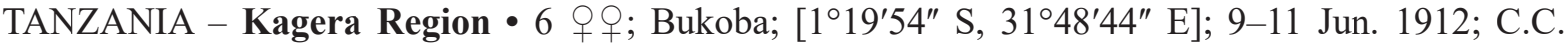
Gowdey leg.; BMNH.

TOGO - Centrale Region • 1 ㅇ; Bismarckburg [near Yégué]; [ $8^{\circ} 10^{\prime} 32.74^{\prime \prime}$ N, 0 $0^{\circ} 41^{\prime} 09.42^{\prime \prime}$ E]; 23-26 Nov. 1892; L. Conradt leg.; ZMB • 1 क ; same collection data as for preceding but 28 May-12 Jun 1893; ZMB $\bullet 1$ \%; same locality as for preceding; R. Büttner leg.; ZMB.

UGANDA • 1 , 1 क; Toro, Daro or Durro Forest; 25-29 Oct. 1911; S.A. Neave leg.; BMNH. - Central Region - 1 کౌ; "Entebbe, Forest within 4 mi. of Kitabi Hill”; 20-25 May 1912; C.A. Wiggins leg.; OUMNH • 1 ; ; same collection data as for preceding but 29 Jun. 1912; OUMNH 1 1 ; ; same collection data as for preceding but Sep. 1913; OUMNH • 1 9 ; same collection data as for preceding but Oct. 1913; OUMNH • 1 O ; Bweya; [0¹1'39" N, 32³2'59" E]; 10 May 1913; C.C. Gowdey leg.; BMNH •

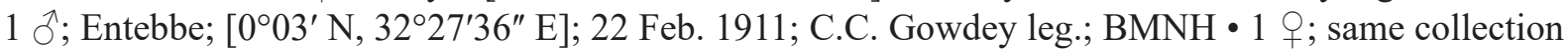
data as for preceding but 26 Aug. 1911; BMNH $\bullet 1$ ग, 4 우우; same collection data as for preceding but 14-25 May 1912; BMNH - 1 क; same collection data as for preceding but 30-31 May 1912; BMNH • 1 ; ; same collection data as for preceding but Sep. 1912; BMNH • 1 क ; same locality as for preceding; 10 Apr. 1912; C.A. Wiggins leg.; OUMNH • 1 § ; same collection data as for preceding but 11 Apr. 1912; OUMNH -2 우; same collection data as for preceding but 26 May 1912; OUMNH • 1 \%; Mabira;

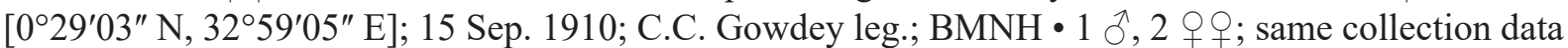
as for preceding but $20 \mathrm{Sep} .1910$; BMNH $1 \mathrm{O}^{\top}, 1$ q; Mawakota; [0³0' N, 32॰15' E]; Jul. 1928; V.G.L. van Someren leg.; BMNH • 1 q; Mpanga Forest, Mpigi; [0 $0^{\circ} 12^{\prime} 33^{\prime \prime}$ N, 32 $2^{\circ} 17^{\prime} 44^{\prime \prime}$ E]; 25-30 Nov. 2014;

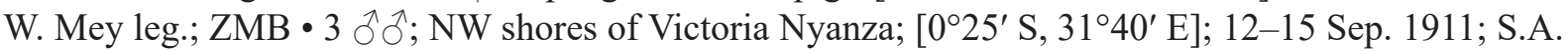
Neave leg.; BMNH • 1 क; Tero Forest; [ $0^{\circ} 50^{\prime}$ S, 31 ${ }^{\circ} 40^{\prime}$ E]; Jul. 1912; C.C. Gowdey leg.; BMNH 1 क ; Tero Forest, SE of Buddo; [0 $0^{\circ} 50^{\prime}$ S, 31 ${ }^{\circ} 40^{\prime}$ E]; 26-30 Sep. 1911; S.A. Neave leg.; BMNH. - Western Region • 1 ; ; 20-50 km NEE of Fort Portal; [042'29.4" N, 30³5'13.1" E]; 26 Nov. 2001; M. Snižek leg.; THD-023-OOLM; GenBank CO1 gene: MW538563; OÖLM • 5 우; Unyoro, Budongo Forest; [14' N, 3134' E]; 11-15 Dec. 1911; S.A. Neave leg.; BMNH.

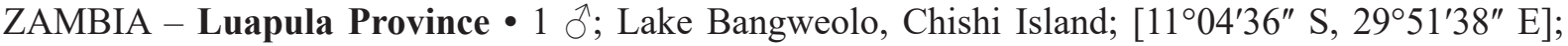
23-25 Jun. 1908; S.A. Neave leg.; OUMNH.

\section{Description}

Female

Size. 28.1-32.2 mm.

CoLOR. Black except for dark ferruginous stripe in center of mandible. Wings fuscous, with violet luster. 

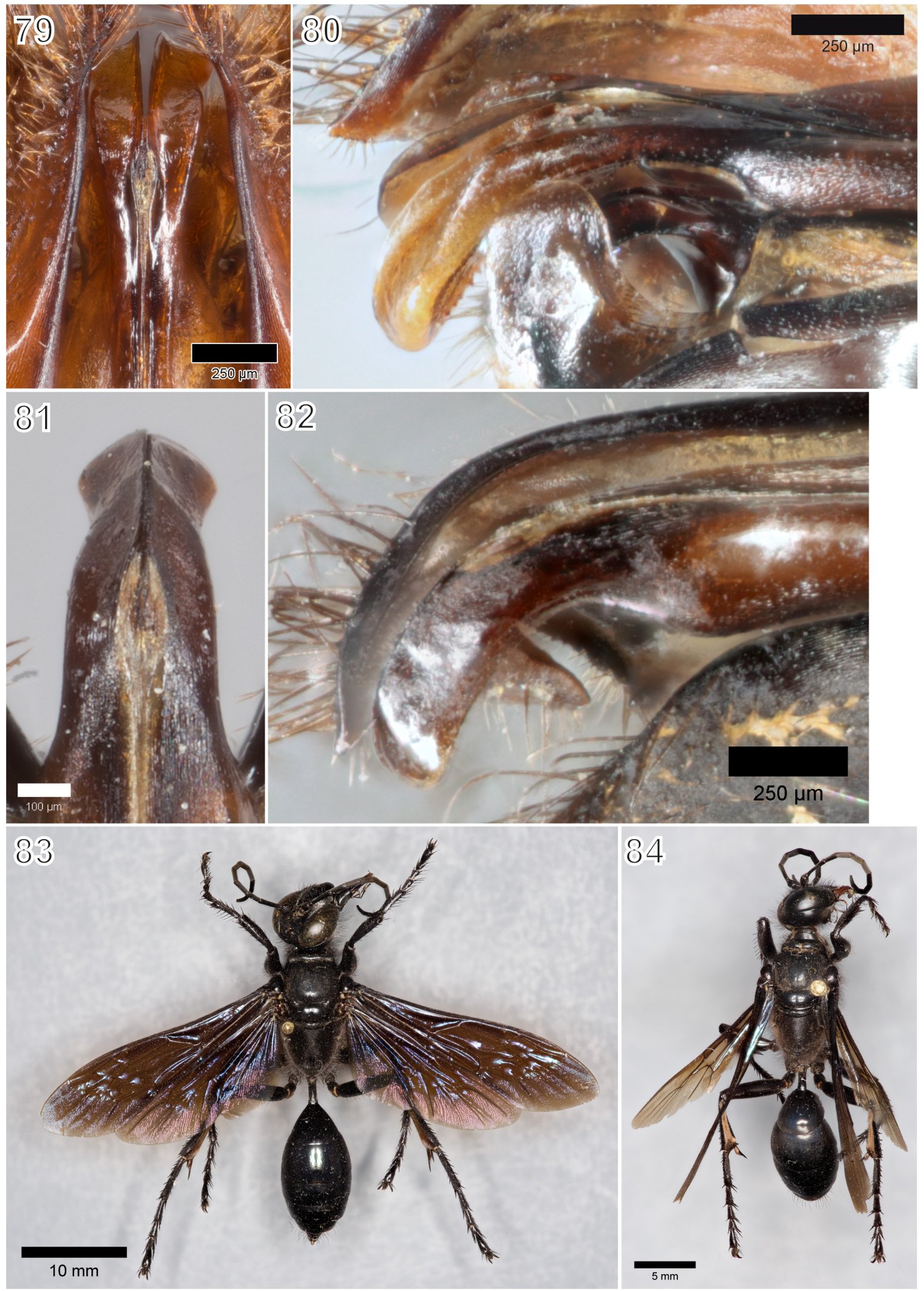

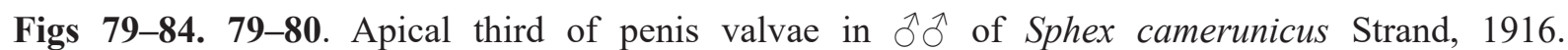
81-82. Apical third of penis valvae in $\hat{\partial}$ of $S$. nigrohirtus Kohl, 1895. 79, 81. Dorsal view. 80, 82. Lateral view. 83. S. camerunicus, habitus of $q$. 84. S. nigrohirtus, habitus of $q$. 
VeSTITURE. Appressed setae on clypeus, paraocular area and collar silvery, on scutum and propodeal enclosure black. Erect setae on clypeus, paraocular area, collar, scutum and propodeal enclosure black. Erect propodeal setae oriented posteriorly. Central third of clypeus glabrous. Scutellum densely and coarsely pubescent.

StruCture. Free clypeal margin with broad, shovel-like process medially, slightly stepped above. Clypeus with notable indentation in lower center and longitudinal carina above. Scutellum convex. Metanotum not raised, not bituberculate. $2^{\text {nd }}$ recurrent vein joins markedly proximal from interstitium between submarginal cells II and III. Propodeal enclosure without any notable ridges. Foretarsomere I 2.9-3.2 $\times$ length of antepenultimate spine. Petiole length 1.9-2.3 $\times$ its medial width.

\section{Male}

SizE. 25.3-30.9 mm.

COLOR. Black except for ferruginous stripe in center of mandible. Wings fuscous, with violet luster.

VeSTITURE. Appressed setae on clypeus, paraocular area and collar silvery, on scutum and propodeal enclosure black. Erect setae on clypeus, paraocular area, collar, scutum and propodeal enclosure black, on paraocular area intermixed with silvery ones. Erect propodeal setae oriented posteriorly. Central third of clypeus glabrous. Scutellum densely and coarsely pubescent. Metasomal sterna III-VI with increasingly dense fringes of black setae.

Structure. Free clypeal margin simple. Scutellum convex. Metanotum not raised, not bituberculate. $2^{\text {nd }}$ recurrent vein joins markedly proximal from interstitium between submarginal cells II and III. Propodeal enclosure without any notable ridges. Posterior margin of metasomal tergum VII convex. Posterior margin of metasomal sternum VII simple, of metasomal sternum VIII triangular. Penis valvae fused, inner margin flattened and dorsally raised in its apical fourth, outer margin more broadly raised. Petiole length 2.0-2.3 $\times$ its medial width. Flagellomeres IV-VII with broad placoids covering their entire length.

\section{Variation}

Unknown.

\section{Distribution}

Western and central Africa.

\section{Remarks}

This species and its synonym $S$. conradti were incorrectly synonymized with $S$. nigrohirtus by Arnold $(1928,1951)$ and Berland (1952). Our study of the types revealed that $S$. camerunicus is actually a valid species. The corresponding male is described here for the first time.

Sphex incomptus Gerstaecker, 1871

Figs 85, 91 (red)

Sphex incomptus Gerstaecker, 1871: 352, $\widehat{0}$.

Chlorion nyanzae R. Turner, 1918: 358, ㅇ, Ô. Synonymized with Chlorion incomptum by Arnold 1928: 368.

\section{Differential diagnosis}

The members of this species are recognizable through having the majority of the propodeal setae silvery (Fig. 85). Excepting the unmistakable $S$. ahasverus, which possesses rich golden vestiture, the other 
females in the nigrohirtus group have predominantly black erect propodeal setae (Figs 83-84, 86). Males of S. pulawskii sp. nov. have some of the propodeal setae silvery, but are differentiated through their conspicuous purple wing iridescence (Fig. 87).

\section{Material examined}

Holotype or syntype

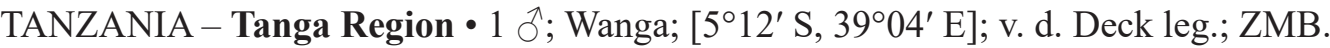

\section{Other material}

AFRICA $・ 1$; 26 Mar. 1912; C.A. Wiggins leg.; OUMNH.

ANGOLA • 1 q; Welwilsch leg.; BMNH. - Húlla Province • 1 q; 1886; Campana leg.; MNHN.

CAMEROON - Southwest Region • 1 क; Malende Mukuya, S of Kumba; [ $4^{\circ} 21^{\prime 2} 25^{\prime \prime}$ N, 9²5'58' E]; 17 May 2006; J. Oehlke leg.; coll. Jacobs.

DEMOCRATIC REPUBLIC OF THE CONGO • 2 우; "Sankuru"; 1910; Abrassart leg.; MRAC • 1 q; Lomami, Kabwe; Jul.-Aug. 1931; P. Quarré leg.; MRAC. - Bas-Uele • 1 क; Bambesa; [32ㅇ' N,

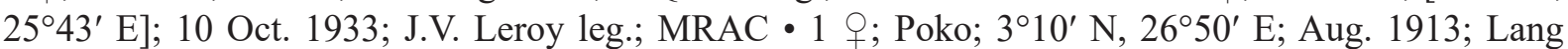

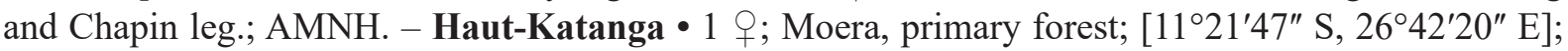
1910; Grauer leg.; NHMW. - Ituri Province • 1 क; Bunia; [1³4' N, 30¹5’ E]; 1938; P. Lefèvre leg.; MRAC • 1 + ; same locality as for preceding; 1 Feb. 1934; J.V. Leroy leg.; MRAC. - Kasaï • 1 \%; Ikeke;

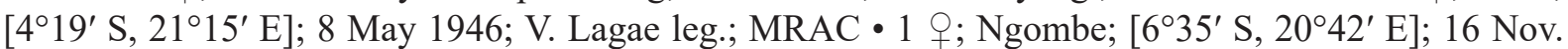
1921; H. Schouteden leg.; MRAC. - Kongo Central • 1 o; Mangembo; [ $4^{\circ} 35^{\prime} 04^{\prime \prime}$ S, $14^{\circ} 16^{\prime} 20^{\prime \prime}$ E];

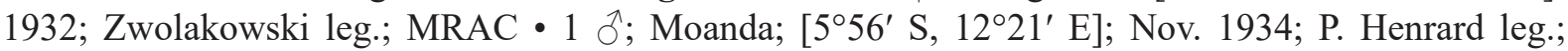

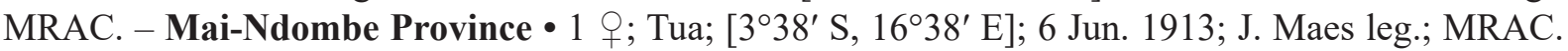

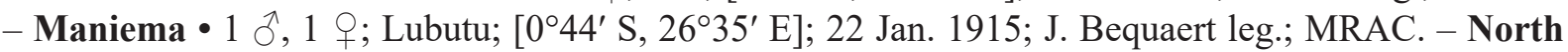

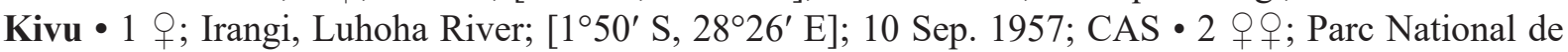
Virungas, Oycha region; Apr.-May 1950; J. de Wilde leg.; MRAC • 1 क; Ukaika; [0 $\left.30^{\prime} \mathrm{N}, 29^{\circ} 00^{\prime} \mathrm{E}\right]$; Dec. 1910; Grauer leg.; NHMW. - Province of Équateur • 1 o ; Eala; [002'30" N, $18^{\circ} 20^{\prime} 06^{\prime \prime}$ E]; 6 Oct. 1931; H.J. Brédo leg.; MRAC • 1 §̊; same collection data as for preceding but 19 Oct. 1931; MRAC •

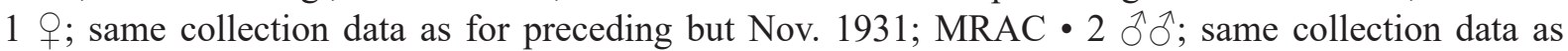
for preceding but 22 Nov. 1931; MRAC $\bullet 1$ ऊं; same collection data as for preceding but Mar. 1932; MRAC $\bullet 1 \hat{\partial}$; same collection data as for preceding but May 1932; MRAC $\bullet 2 \hat{\jmath} \bar{\partial}$; same locality as for preceding; Jun. 1932; A. Corbisier leg.; MRAC • 1 \%; same collection data as for preceding but 7 Jul. 1932; MRAC • 1 ; ; same collection data as for preceding but Nov. 1932; MRAC • 1 \% ; same collection data as for preceding but Dec. 1932; MRAC $\bullet 1$; ; same collection data as for preceding but 14 Mar.

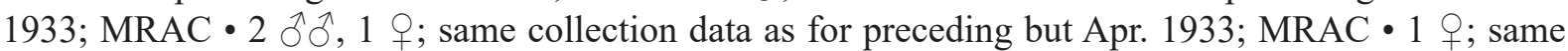
locality as for preceding; May 1935; J. Ghesquière leg.; MRAC. - Sankuru • 1 क; Kondue; [45ㅇ' S, $23^{\circ} 16^{\prime}$ E]; Leonhard leg.; MRAC. - Tshopo 1 क; Stanleyville [now Kisangani]; $0^{\circ} 30^{\prime} \mathrm{N}, 25^{\circ} 10^{\prime}$ E; 10 Apr. 1915; Lang and Chapin leg.; AMNH • 1 O ; Stanleyville [now Kisangani]; $0^{\circ} 30^{\prime}$ N, $25^{\circ} 10^{\prime}$ E; 11 Apr. 1915; Lang and Chapin leg.; AMNH. - Tshuapa • 1 q; Bokuma; [0 $\left.00^{\circ} \mathrm{S}, 18^{\circ} 41^{\prime} \mathrm{E}\right]$; Jul. 1952; R.P. Lootens leg.; MRAC.

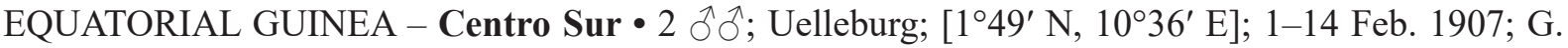
Tessmann leg.; ZMB.

GABON - Estuaire Province • 1 đ̊; Crystal Mountains, Muni; [049'45.6" N, 1008'5.7" E]; 15-31 Oct. 1969; A. Villiers leg.; MNHN • 1 क ; Libreville; [0²3'24.36" N, 9²7'15.84" E]; MNHN. 
REPUBLIC OF THE CONGO - Brazzaville Department • 1 \%; Djoumouna, Yaka-Yaka; [4²2'34" S, 1509'36" E]; 3 Feb. 1977; S. Kellner-Pillaut leg.; MNHN. - Kouilou Department • 1 q; Dimonika; [4¹1'55.8" S, 12²1'19.7" E]; Jan. 1964; A. Descarpentries and A. Villiers leg.; MNHN•1 1 ; same locality as for preceding; 18 May 1977; J.-P. Grillot and C. Morin leg.; MNHN. - Lékoumou Department • 1 q; Ogooué River; [2²6'40.7" S, 13²4'29.4" E]; 1911; F. Faure leg.; MNHN.

TANZANIA - Kagera Region • 1 + , paratype of Chlorion nyanzae R. Turner, 1918; Bukoba; [1 ${ }^{\circ} 19^{\prime} 54^{\prime \prime}$ S,

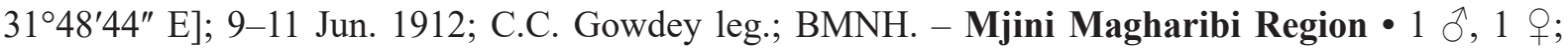
Kizimbani; [903'42.5" S, 39¹9'3.1" E]; 15 Jun. 1985; G.G.M. Schulten leg.; RMNH • 1 ô; Zanzibar;

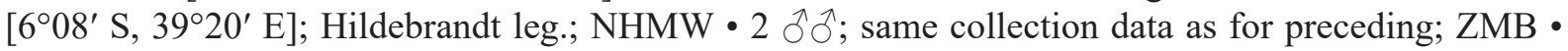
1 q; same locality as for preceding; Raffray leg.; NHMW. - Unguja South Region • 1 đ̇; Zanzibar, Jambiani; [6 $6^{\circ} 19^{\prime}$ S, 39³3' E]; 23 Mar. 1993; K.M. Guichard leg.; BMNH • 2 ô $0^{\top}$; Zanzibar, Mzingani Forest; [6¹0' S, 39¹8' E]; 28 Mar. 1993; K.M. Guichard leg.; BMNH.

UGANDA - Central Region • 1 q; "Entebbe, Forest within 4 mi. of Kitabi Hill”; Jan.-Feb. 1913; C.A.

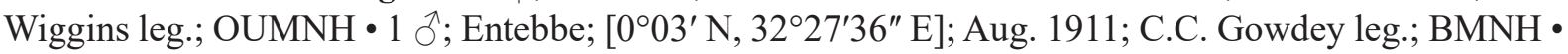
1 q; same collection data as for preceding but 14-25 May 1912; BMNH 1 क ; same collection data as for preceding but 1-4 Jun. 1912; BMNH -5 우; same collection data as for preceding but Aug. 1912; BMNH • 1 \%; same collection data as for preceding but Oct. 1912; BMNH $\bullet 1$; ; same locality as for preceding; 12-20 Jan. 1912; S.A. Neave leg.; BMNH 1 ô, holotype of Chlorion nyanzae R. Turner, 1918; same collection data as for preceding; BMNH • 1 क; Mabira; [0 $\left.0^{\circ} 9^{\prime} 03^{\prime \prime} \mathrm{N}, 32^{\circ} 59^{\prime} 05^{\prime \prime} \mathrm{E}\right]$; 15 Sep.

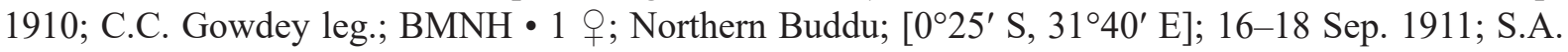

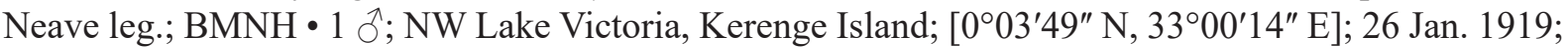

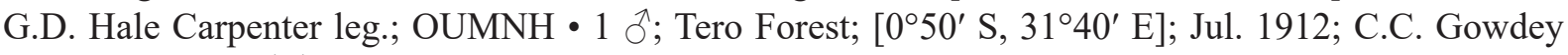

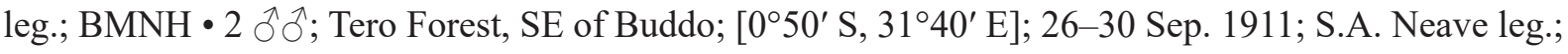
BMNH. - Western Region • 1 q; Kabarole Kibale Forest National Park, Kanyawara (MUBFS); 0 $33^{\prime}$ N, 30²1' E; 23 Aug. 1997; C. Häuser leg.; ZMB • 1 đ’; Kichwamba, Ankole; [0¹3'08.2" S, 3005'50.3" E]; 1-5 May 1968; P.J. Spangler leg.; USNM • 1 ㅇ; near Rwera, SW of Hoima; [1 ${ }^{\circ} 02^{\prime} 25^{\prime \prime}$ S, 30¹8'47" E]; 30 Nov. 2001; M. Snižek leg.; OÖLM.

ZIMBABWE - Mashonaland Central - 1 ; ; Mavhuradonha Saf., $15 \mathrm{~km}$ SE of Muzarabani; [16³3'33" S, 3114'22.8" E]; 17 Dec. 1998; J. Halada leg.; THD-035-OOLM; GenBank LWR gene: MW582292; OÖLM.

\section{Description}

Female

SizE. $23.7-25.7 \mathrm{~mm}$.

COLOR. Black except for basal half of mandible and free clypeal margin, which are ferruginous.

ForEwING. Slightly fuscous, with violet iridescence. Basal half of hindwing hyaline, apical margin slightly infuscate.

VeSTITURE. Appressed setae on clypeus, paraocular area, collar and propodeal enclosure silvery, on scutum laterally silvery, otherwise black. Erect setae on clypeus and paraocular area black, on collar silvery intermixed with black ones, on scutum black intermixed with silvery ones, on propodeal enclosure silvery. Erect propodeal setae oriented posteriorly. Lower center of clypeus glabrous. Scutellum densely and coarsely pubescent.

StRUCTURE. Free clypeal margin with broad, shovel-like process medially, not stepped above. Clypeus with slight indentation in lower center and longitudinal carina above. Scutellum convex. Metanotum not raised, not bituberculate. $2^{\text {nd }}$ recurrent vein joins markedly proximal from interstitium between 


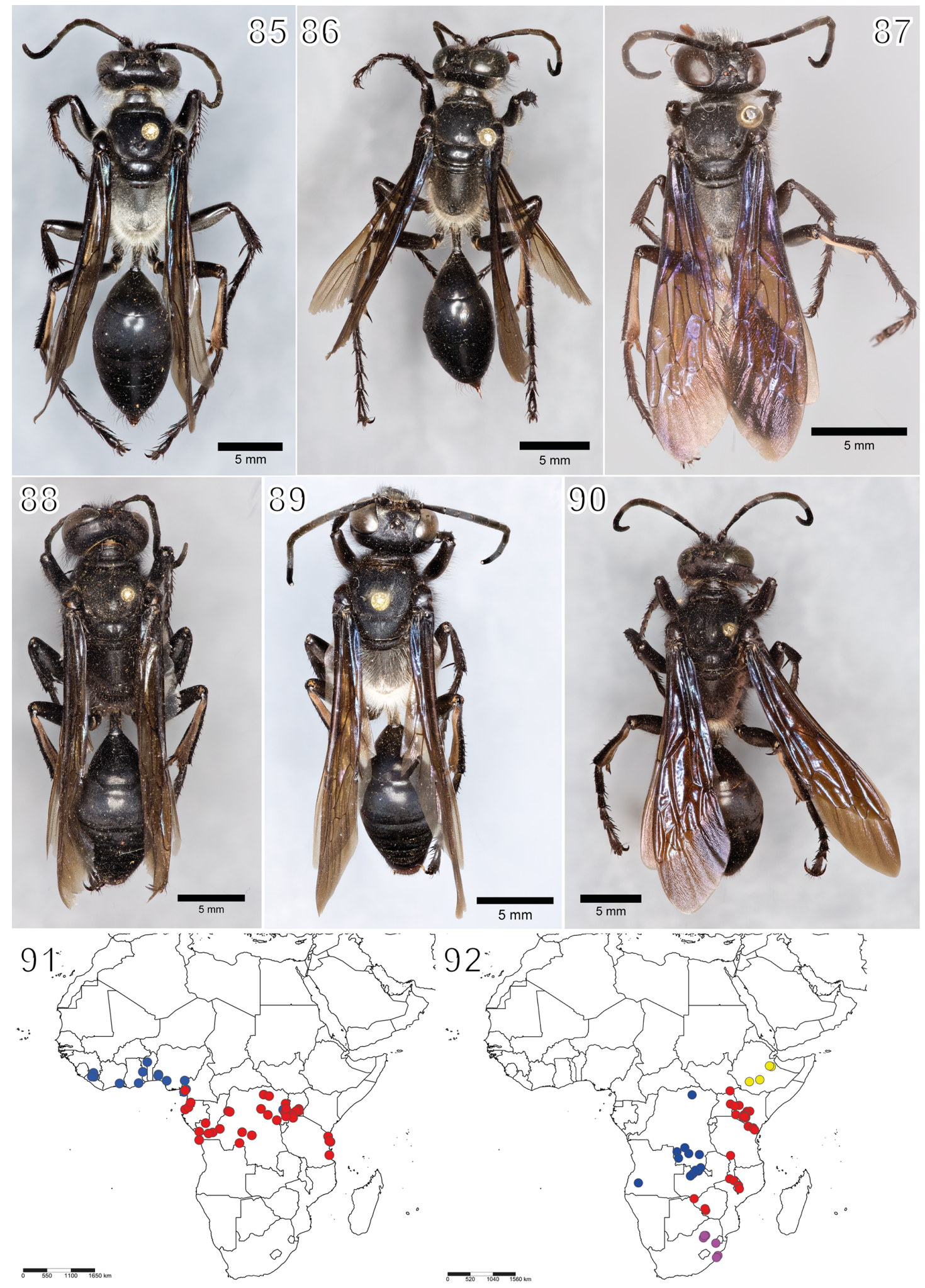

Figs 85-92. 85-86. Habitus of $q$. 87-90. habitus of $\widehat{\jmath}$. 85. Sphex incomptus Gerstaecker, 1871. 8687. S. pulawskii sp. nov. 88. S. abbotti abbotti W. Fox, 1891. 89. S. abbotti nivarius subsp. nov. 90. S. bohemanni Dahlbom, 1845. 91. Geographic distribution of S. incomptus (red) and S. pulawskii sp. nov. (blue). 92. Geographic distribution of $S$. a. abbotti (red), S. a. nivarius subsp. nov. (blue), S. abyssinicus (Arnold, 1928) (yellow) and S. bohemanni (purple). 
submarginal cells II and III. Propodeal enclosure without any notable ridges. Foretarsomere I 2.8-3.0× length of antepenultimate spine. Petiole length $1.7-2.3 \times$ its medial width.

Male

SizE. $20.5 \mathrm{~mm}$.

CoLOR. Black except for ferruinous stripe in center of mandible. Forewing slightly fuscous, with violet iridescence. Basal half of hindwing hyaline, apical margin slightly infuscate.

VeSTITURE. Appressed setae on clypeus, paraocular area, collar and propodeal enclosure silvery, on scutum laterally silvery, otherwise black. Erect setae on clypeus, paraocular area and scutum black intermixed with silvery ones, on collar silvery intermixed with black ones and on propodeal enclosure silvery. Erect propodeal setae oriented posteriorly. Lower center of clypeus glabrous. Scutellum densely and coarsely pubescent. Metasomal sterna II-VI with increasingly dense fringes of black setae.

StruCture. Free clypeal margin simple. Scutellum convex. Metanotum not raised, not bituberculate. $2^{\text {nd }}$ recurrent vein joins markedly proximal from interstitium between submarginal cells II and III. Propodeal enclosure without any notable ridges. Posterior margin of metasomal tergum VII gently notched. Posterior margin of metasomal sternum VII simple, of metasomal sternum VIII concavely emarginate. Penis valvae without conspicuous modifications. Petiole length $2.2-2.9 \times$ its medial width. Flagellomere $\mathrm{V}$ with narrow placoid covering its proximal four-fifths and tapering distally.

\section{Variation}

Unknown.

\section{Distribution}

Central to eastern Africa.

\section{Remarks}

There seems to have been some confusion regarding the differentiation between this taxon and the closely related $S$. nigrohirtus, which we were able to clear up through the study of their types. When Kohl (1895) compared them with each other, he correctly stated that one of the few differences between the males of both species is the color of the clypeal and thoracical setae. In S. incomptus, the black erect setae on the clypeus, scutum, scutellum and metanotum are intermixed with silvery ones, while the erect setae on the collar and propodeum are exclusively silvery. In contrast, $S$. nigrohirtus has the erect setae in these areas almost uniformly black. Even though Arnold was apparently aware of this fact and mentioned it in the redescriptions of both species (1928), he nevertheless assigned several specimens with uniformly black vestiture to $S$. incomptus without actually citing any characters to disassociate them from S. nigrohirtus. Later, Leclercq (1955) would continue this path with his establishment of the subspecies $S$. incomptus anonymus for specimens with only black pilosity. Our study of the types showed that they are actually members of $S$. nigrohirtus. Subsequently, specimens of $S$. nigrohirtus were prone to be misidentified as belonging to $S$. incomptus, which is evident in the material examined by Dollfuss (2008), where most of the locality records given for $S$. incomptus in reality correspond to S. nigrohirtus.

Sphex nigrohirtus Kohl, 1895

Figs 13, 78 (blue), 81-82, 84

Sphex nigrohirtus Kohl, 1895: 66, §.

Sphex incomptus anonymus Leclercq, 1955: 12, §. Syn. nov. 


\section{Differential diagnosis}

Females of this taxon are easily recognizable, as they differ from all others in the group through having the mesosomal setae uniformly black (Fig. 84). Those of S. camerunicus (Fig. 83), S. incomptus (Fig. 85) and S. pulawskii sp. nov. (Fig. 86) have at least the appressed setae posteriorly on the collar and at a spot posterolaterally on the scutum silvery. Males of S. nigrohirtus can be more difficult to identify, as not all of them have uniformly black mesosomal setae. Those of $S$. camerunicus are distinguished through their dorsolaterally concave valvicepes (Figs 79-80) and broad placoids on flagellomeres IVVII, whereas S. nigrohirtus has the valvicepes dorsolaterally flat or convex (Figs 81-82) and usually only a narrow placoid on flagellomere V. Sphex incomptus differs in having predominantly silvery propodeal setae and only slightly infuscate wings. Finally, males of S. pulawskii sp. nov. can be differentiated from this species through their conspicuous purple wing iridescence without a cyan tone (Fig. 87), while S. nigrohirtus always has cyan iridescence, at least on the basal half of the forewing.

\section{Material examined}

Lectotype (designated here)

TANZANIA - Tanga Region - đ̄; "Usambara and Bondei”; Feb.-Mar. 1880; C.W. Schmidt leg.; ZMB.

\section{Paralectotype}

TANZANIA $\bullet 1$; same collection data as for lectotype; ZMB.

\section{Other material}

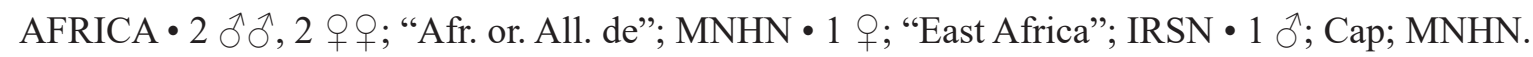

DEMOCRATIC REPUBLIC OF THE CONGO-Haut-Katanga • 1 今 ; Elisabethville [now Lubumbashi];

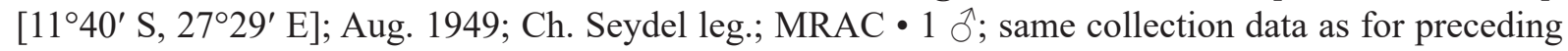
but 30 Sep. 1949; MRAC - 3 q $O$; same collection data as for preceding but 24 Jun. 1951; MRAC • 2 우; same collection data as for preceding but 17 May 1953; MRAC $\bullet 1$; ; same collection data as for preceding but May 1953; MRAC. - Haut-Lomami 1 o , holotype of Sphex incomptus anonymus Leclercq, 1955; "Upemba National Park, Kankunda (rive dr. Lupiala)"; [8³0' S, 26³0' E]; alt. 1300 m; 13-27 Nov. 1947; G.F. de Witte leg.; MRAC. - Lualaba - 1 q; Kapanga; [8 ${ }^{\circ} 21^{\prime}$ S, 22 $2^{\circ} 34^{\prime}$ E];

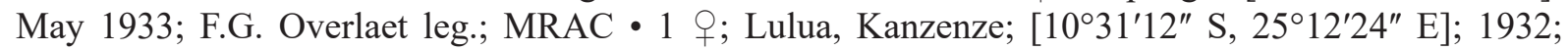
R.P. Lefebure leg.; MRAC.

ETHIOPIA - Oromia Region • 1 §̧; Hawash River, S of Adama; 7 Jun. 1946; K.M. Guichard leg.; BMNH • 1 क; near Wachile; 432' N, 3903' E; 30-31 May 2015; J. Halada leg.; OÖLM. - Southern

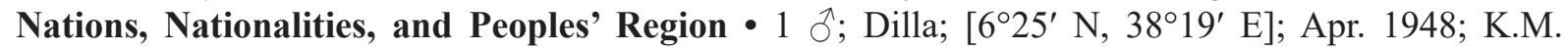
Guichard leg.; BMNH.

KENYA • 3 q $q$; Mogorr River; May 1913; A.O. Luckman leg.; BMNH. - Homa Bay County • 1 ; Ungoye, ICIPE Field Station; $0.61325^{\circ}$ S, 34.8908 E; 13-27 Apr. 2006; R. Copeland leg.; ICIPE • 1 우; Ungoye, ICIPE Field Station, inside seasonally swampy forest; $0.61517^{\circ} \mathrm{S}, 34.9200^{\circ} \mathrm{E} ; 22$ Jun.-6 Jul. 2006; R. Copeland leg.; ICIPE. - Kajiado County • 1 o ; Ngong; [1²1'09" S, 36 40'11" E]; Apr. 1941; V.G.L. van Someren leg.; BMNH. - Kilifi County • 1 +; Gedi National Park; [3¹8'33.84" S, 4001'1.92" E]; 2-6 Nov. 1972; M. Boulard leg.; MNHN • 1 O; Rabai; [355'54" N, 39³4'13" E]; Apr. 1928; V.G.L. van Someren leg.; BMNH • 2 ๙ิ ${ }^{\lambda}, 1$ q; same collection data as for preceding but Nov.-Dec. 1933; BMNH. - Kirinyaga County • 1 क ; Njuki-ini Forest, near Forest station; $0.51660^{\circ} \mathrm{S}$, 37.41843 ${ }^{\circ}$ E; 30 Jan.-12 Feb. 2007; R. Copeland leg.; ICIPE. - Kitui County • 1 ; ; Kasaala area; $2.7836^{\circ} \mathrm{S}, 38.22517^{\circ} \mathrm{E}$; 3-17 May 2016; R. Copeland leg.; ICIPE • 1 q; Ngaia Forest, bottom of forest; $0.32442^{\circ}$ S, $38.5038^{\circ}$ E; 24 Dec. 2011-7 Jan. 2012; R. Copeland leg.; ICIPE. - Kwale County • 1 ';

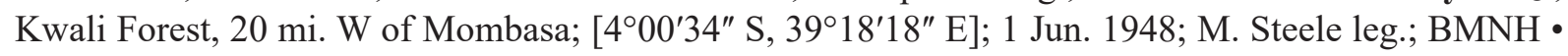




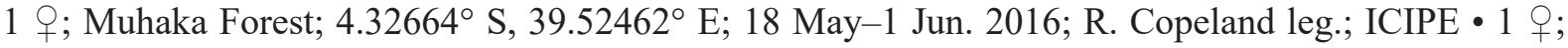
Shimba Hills; 4¹2.3' S, 39²5.2' E; 27 Jun. 1999; W.J. Pulawski and J.S. Schweikert leg.; CAS • 1 ô, 1 क; same locality as for preceding; 15 Dec. 2003; W.J. Pulawski leg.; CAS. - Meru County • 1 क ; Meru; [002'46" N, 37³9'21" E]; Sep. 1927; V.G.L. van Someren leg.; BMNH • 1 ô; same collection data as for preceding but Jul. 1943; BMNH. - Muranga County • $3 \hat{\jmath} \delta^{2}, 3$ 우; E foot and slopes of Aberdare Mts; [0³8'21" S, 36²7'28" E]; 24-27 Feb. 1911; S.A. Neave leg.; BMNH. - Nyeri County •

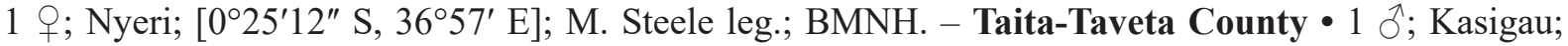
[349' S, 3840' E]; Nov. 1938; V.G.L. van Someren leg.; BMNH • 1 क; Mwatate area, below Bura Bluff, riverine forest; $3.48444^{\circ} \mathrm{S}, 38.33251^{\circ} \mathrm{E}$; 7-21 Feb. 2012; R. Copeland leg.; ICIPE • 1 क ; Voi; [3²3'26" S,

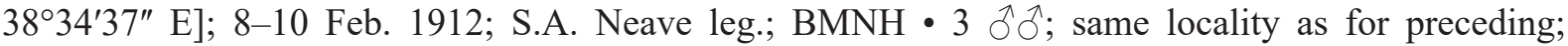

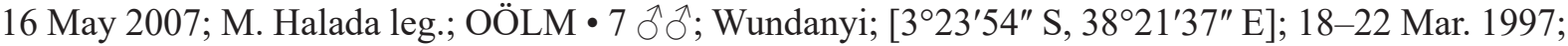

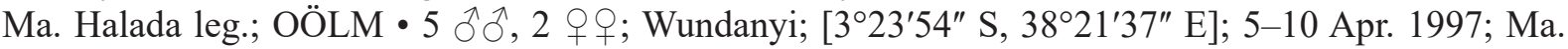
Halada leg.; OÖLM. - Trans-Nzoia County • 1 \%; Mt Elgon E side, Elgon Saw mill; [1 ${ }^{\circ} 05^{\prime} 19.6^{\prime \prime} \mathrm{N}$, 3445'15.5" E]; 1932-1933; C. Arambourg, P.-A. Chappuis and R. Jeannel leg.; MNHN.

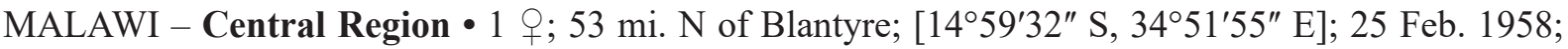

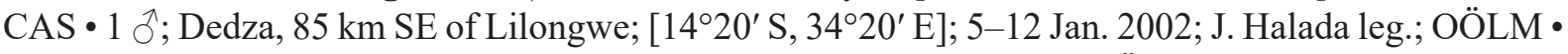
1 o; Nkhotakota; [12 $56^{\prime} \mathrm{S}, 34^{\circ} 18^{\prime} \mathrm{E}$ ]; 2 Jan. 2002; J. Halada leg.; OÖLM. - Northern Region • 1 क; Karonga; [956' S, 3356' E]; 7-11 Jul. 1910; S.A. Neave leg.; BMNH. - Southern Region • 1 q; Btwn. Ft. Mangoche and Chikala Boma; 20-25 Mar. 1910; S.A. Neave leg.; BMNH 11 ○; Mlanje; [1601'53" S, 35³0' E]; 8-9 Feb. 1912; S.A. Neave leg.; BMNH • 1 क; same collection data as for preceding but 12 Feb. 1912; BMNH 1 o ; same collection data as for preceding but 23 Feb. 1912; $\mathrm{BMNH} \bullet 1$ \%; same collection data as for preceding but $26 \mathrm{Feb} .1912 ; \mathrm{BMNH} \bullet 1$ q; same collection data as for preceding but 3 Dec. 1912; BMNH $\bullet 1$ त; same collection data as for preceding but 4 Dec. 1912; BMNH • 1 ; ; same collection data as for preceding but 14 Dec. 1912; BMNH 2 \% $ᄋ$; same collection data as for preceding but 27 Dec. 1912; BMNH • 3 $\lesssim$; ; same collection data as for preceding but 6 Jan. 1913; BMNH 1 1 ; same collection data as for preceding but 7 Jan. 1913; BMNH $\bullet$ 2 Q $ᄋ$; same collection data as for preceding but $15 \mathrm{Jan} .1913$; BMNH $\bullet 1$ \%; same collection data as for preceding but $17 \mathrm{Jan} .1913$; BMNH $\bullet 1$; ; same collection data as for preceding but 21 Jan. 1913; BMNH • 1 \%; same collection data as for preceding but 27 Jan. 1913; BMNH • 1 \%; same collection data as for preceding but $1 \mathrm{Feb} .1913$; BMNH 1 1 ; same collection data as for preceding but 4 Feb. 1913; BMNH • 2 우; same collection data as for preceding but 11 Feb. 1913; BMNH • 1 o ; same collection data as for preceding but $20 \mathrm{Feb} .1913$; BMNH $\bullet 1$ 万; same collection data as for preceding

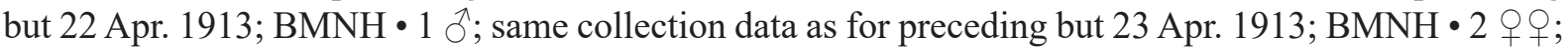
same collection data as for preceding but $5 \mathrm{Sep} .1913$; BMNH $\bullet 1$ O , 1 क ; same collection data as for preceding but 7 Nov. 1913; BMNH $\bullet 1$ ग, 1 \%; same collection data as for preceding but 6 Dec. 1913; $\mathrm{BMNH} \bullet 1$ \%; same collection data as for preceding but 21 Dec. 1913; BMNH 11 ; ; same collection data as for preceding but $10 \mathrm{Feb} .1914$; BMNH 1 ते; same collection data as for preceding but 16 Feb. 1914; BMNH • 1 q; Mlanji Boma; [1601'53" S, 35³0' E]; 26-30 Apr. and 3-5 May 1910; S.A. Neave

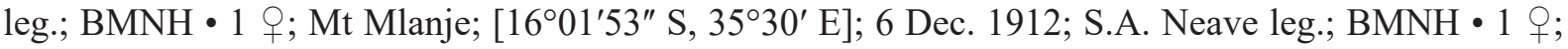

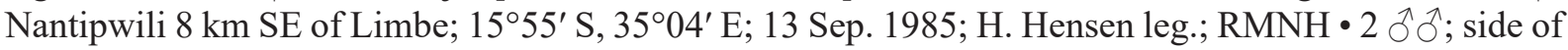

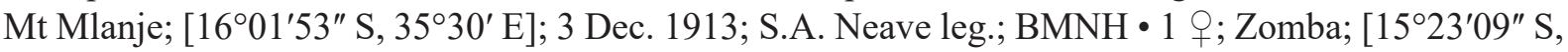
$35^{\circ} 19^{\prime} 07^{\prime \prime}$ E]; Dec. 1894; BMNH.

MOZAMBIQUE • 1 § , 1 क; valley of Kola River, near E Mt Chiperone; 6 Apr. 1913; S.A. Neave leg.; $\mathrm{BMNH} \bullet 1$ Oे, 1 ; ; same collection data as for preceding but 21 Nov. 1913; BMNH. - Manica Province •

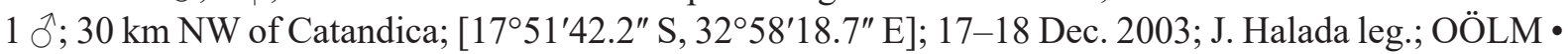
1 ㅇ; Zambezi, near Tambara, Cangare Lake; [1657'36.67" S, 3405'2.23" E]; 3 May 1929; P. Lesne leg.; MNHN. - Sofala Province • 1 q; near Beira, Manga; [1950' S, 34²51' E]; 27 Jul. 1929; P. Lesne leg.; 


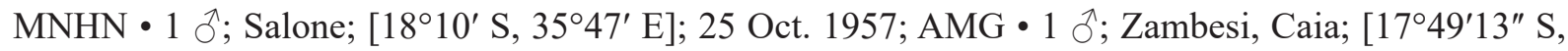
3520'39" E]; 18 Apr. 1912; H. Swale leg.; BMNH.

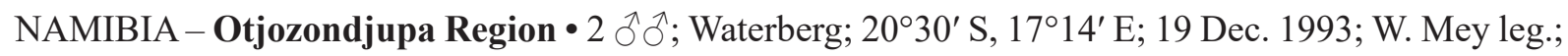
ZMB.

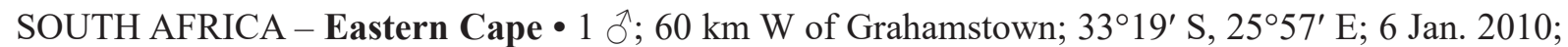

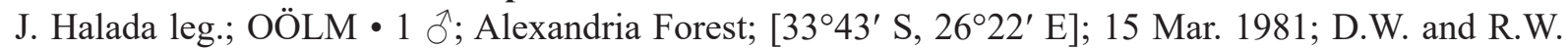

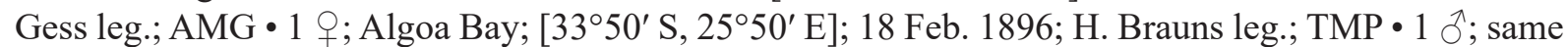
collection data as for preceding but $23 \mathrm{Feb} .1896$; TMP $\bullet 1$; same collection data as for preceding;

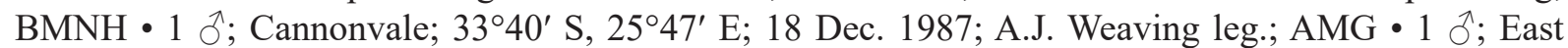

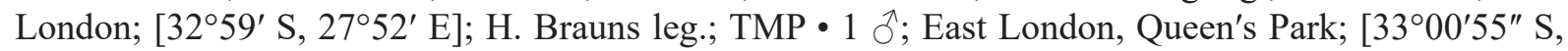

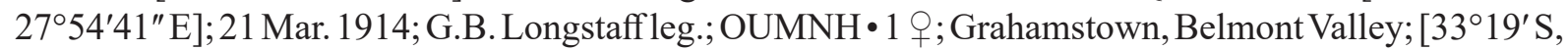

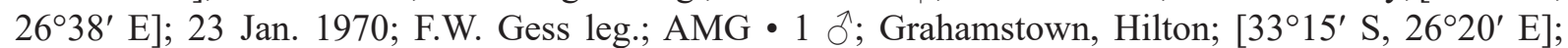

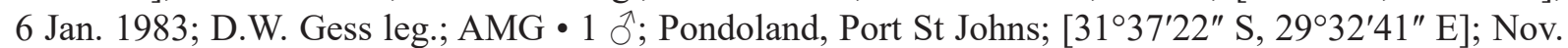
1923; R.E. Turner leg.; BMNH • 2 ôं; same collection data as for preceding but 6-25 Feb. 1924;

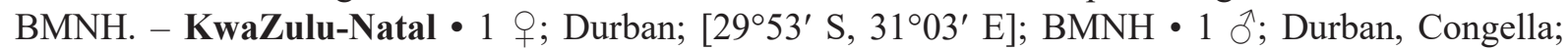

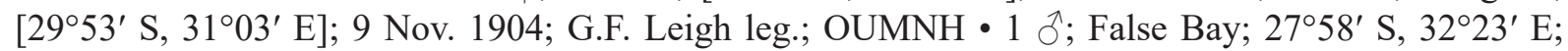
9-11 Mar. 1990; A.J. Weaving leg.; AMG • 1 o $^{\top}$; Ithala Game Res., Savannah; 2730' S, 31 $20^{\prime}$ E; 4-10

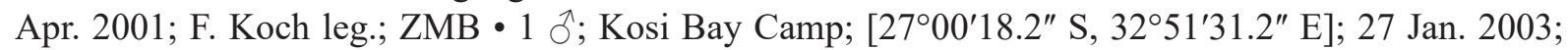

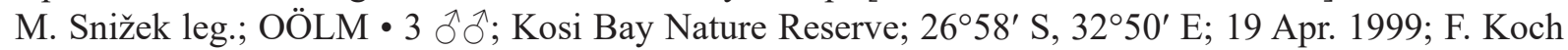

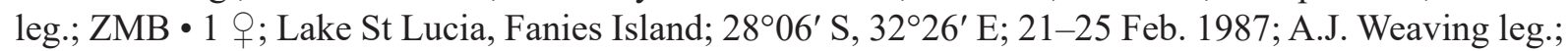

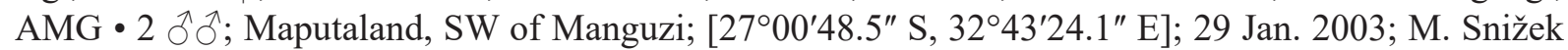

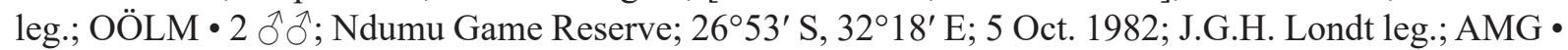

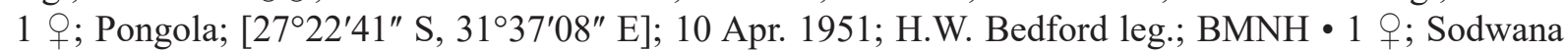
Bay National Park; $27^{\circ} 37^{\prime}$ S, 32 $2^{\circ} 41^{\prime}$ E; 1 Feb. 1994; F. Koch leg.; ZMB • 1 Oे; same collection data as for preceding but 7-10 Apr. 2001; ZMB • 1 O ; St Michaels; 3049'121" S, 30²4'119" E [sic]; 14 Jan.

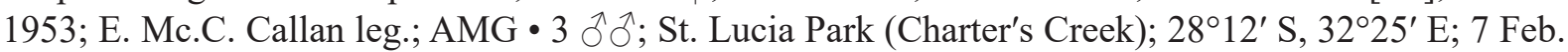

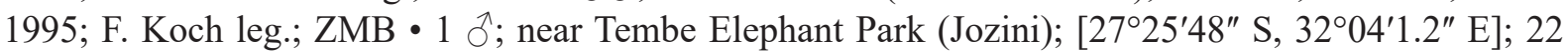
Jan. 2003; V. Krivan leg.; OÖLM • 1 ơ ; Umlalazi River near Mtunzini; $28^{\circ} 56^{\prime}$ S, $31^{\circ} 15^{\prime}$ E; 2 Oct. 1982;

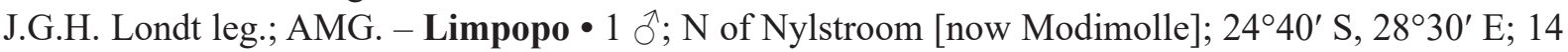
Jan. 2008; M. Halada leg.; THD-013-OOLM; GenBank CO1 gene: MW538567; GenBank LWR gene:

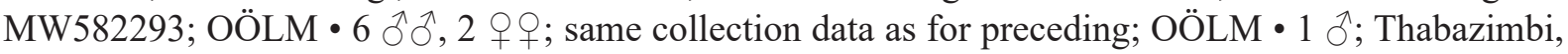

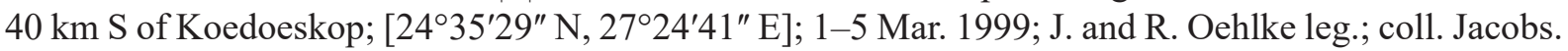

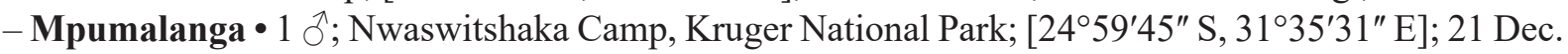
1994; C.L. Bellamy leg.; AMNH. - Western Cape • 2 우; George, 8 mi. E of Silver Kloof; [33 $57^{\prime} 46^{\prime \prime}$ S, $22^{\circ} 27^{\prime} 42^{\prime \prime}$ E]; 28 Feb. 1914; G.B. Longstaff leg.; OUMNH • 1 \%; Knysna, 14 mi. from road to George, Homtini Kloof; [3402'10" S, 2302'49" E]; 24 Feb. 1914; G.B. Longstaff leg.; OUMNH.

TANZANIA • 1 क; "East Africa"; F. Stuhlmann leg.; ZMB • 1 क; "German East Africa"; E. Fischer leg.; ZMB • 1 क; Lake Rukwa, E of Ukimbu; 25-27 Aug. 1899; Glauning leg.; ZMB. - Arusha Region • 4 우; 5 mi. S of Momela Lodge; [3 $3^{\circ} 18^{\prime} 10^{\prime \prime}$ S, 36 $56^{\circ} 51^{\prime \prime}$ E]; 9 Jan. 1970; M.E. Irwin and E.S. Ross leg.; CAS • 1 q; Momela, Mt Meru; $3^{\circ} 15^{\prime}$ S, 36 $56^{\circ}$ E; $12-13$ Jan. 1972; C.F. Huggins leg.; BMNH • 1 क; Momella Lakes, Mount Meru; [ $3^{\circ} 18^{\prime} 10^{\prime \prime}$ S, 36 $6^{\circ} 1^{\prime} 51^{\prime \prime}$ E]; 20-31 Jan. 1964; W. Forster leg.; CAS •

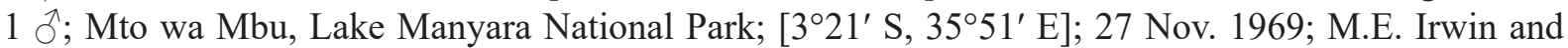
E.S. Ross leg.; CAS. - Dar es Salaam Region - 1 \%; Dar es Salaam; [6 $6^{\circ} 48^{\prime}$ S, 39 $9^{\circ} 17^{\prime}$ E]; Aug.-Sep. 1902; Meinhof leg.; ZMB. - Kilimanjaro Region 1 1 , paralectotype of Sphex kilimandjaroensis Cameron, 1908; Kilimanjaro, Kibonoto; [305'5.9" S, 37²0'38.5" E]; alt. 1300-1900 m; 11 May 1905; Y. Sjöstedt leg.; BMNH $\bullet 1$; ; same collection data as for preceding but 3 May; NRS $\bullet 1$; ; Kihuiro [now Kihurio]; [4²7'53.5" S, 3804'28.4" E]; 15 Jan. 1906; Schröder leg.; ZMB. - Mbeya Region • 


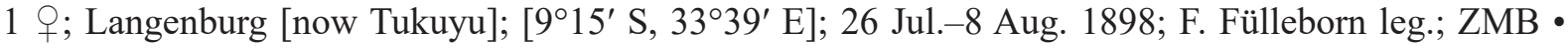

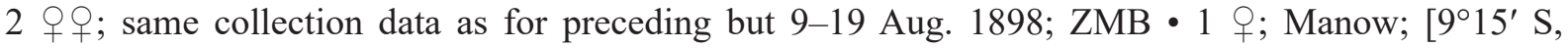
$33^{\circ} 48^{\prime}$ E]; MNHN. - Mjini Magharibi Region - $1 \partial^{\prime}$; Zanzibar; [6 $6^{\circ} 08^{\prime}$ S, $39^{\circ} 20^{\prime}$ E]; ZMB. - Mwanza

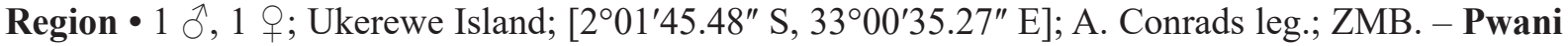

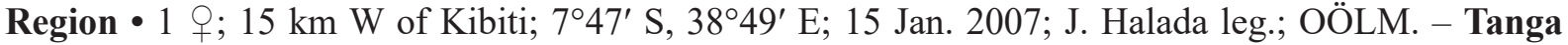

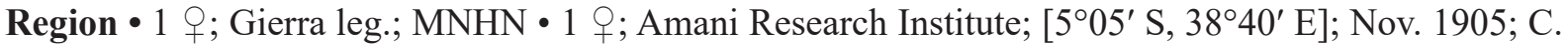
Schröder leg.; ZMB • 1 O ; same locality as for preceding; Feb. 1908; J. Vosseler leg.; ZMB • 1 q; same collection data as for preceding but $20 \mathrm{Feb} .1908 ; \mathrm{ZMB} \bullet 1$ đ'; Usambara, Nguela (Ngwelo); [504'59" S, 3845'59.5" E]; Rolle leg.; ZMB.

UGANDA - Western Region - 1 ऽं; Semliki Plains, near S shore of Lake Albert; [0 $0^{\circ} 59^{\prime} 17^{\prime \prime} \mathrm{N}$, 30³0'09" E]; alt. 2200 ft; 25-27 Nov. 1911; S.A. Neave leg.; BMNH.

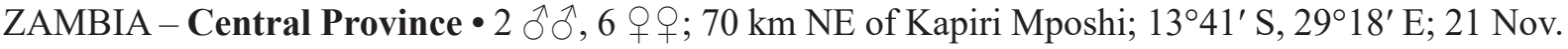

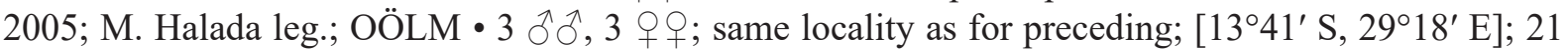
Nov. 2005; M. Snižek leg.; OÖLM • 1 \&; "Mid. Luangwa Valley”; alt. 1700 ft; 28 Feb. 1908; S.A. Neave leg.; OUMNH. - Eastern Province • 1 ○; "Up. Luangwa Valley”; alt. 1800-2000ft; 17 Mar. 1908; S.A. Neave leg.; OUMNH. - Muchinga Province • 2 + ; "L. Chambezi V., Mpika distr.”; alt. 3900 ft; 18 May 1908; S.A. Neave leg.; OUMNH • 1 P; "Mid. Chambezi Valley, Chinsali distr."; alt. 4000 ft; 28 Apr. 1908; S.A. Neave leg.; OUMNH. - Northern Province • 1 क; "High Plateau S Lake Tanganyika (dense forest)"; alt. 4500 ft; 24-25 Aug. 1908; S.A. Neave leg.; OUMNH • 1 q; "L. Chambezi V., Kasama distr.”; alt. 3900 ft; 12-13 May 1908; S.A. Neave leg.; OUMNH • 1 O, paratype of Sphex incomptus anonymus Leclercq, 1955; Abercorn [now Mbala]; [850' S, 31 ${ }^{\circ} 28^{\prime}$ E]; Feb. 1943; IRSN • 1 क; same collection data as for preceding but Oct. 1943; IRSN • 1 \%; E shore of Lake Bangweolo; alt. 3800 ft; 25 May 1908; S.A. Neave leg.; OUMNH. - North-Western Province • 1 ; ; 150 km S of Mwinilunga; $13^{\circ} 11^{\prime}$ S, $24^{\circ} 14^{\prime}$ E; 18 Oct. 2008; M. Halada leg.; OÖLM. - Southern Province • 1 ㅇ Victoria Falls; [1751' S, 2552' E]; 11 Nov. 1967; E.S. Ross and A.R. Stephen leg.; CAS.

ZIMBABWE - Manicaland • 1 \%; Mt Chirinda, Melsetter [now Chimanimani District]; [20 $24^{\prime} 36^{\prime \prime}$ S, $32^{\circ} 40^{\prime} 08^{\prime \prime}$ E]; 6 Oct. 1905; G. Marshall leg.; OUMNH - 1 o; Mt Selinda; [20 25'23.3" S,

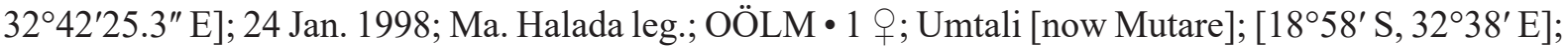
4 Apr. 1931; P.A. Sheppard leg.; TMP. - Mashonaland Central • 6 ô ${ }^{\Uparrow}$; Mavhuradonha Saf., 15 km SE of Muzarabani; [16³3'33" S, 31²14'22.8" E]; 17 Dec. 1998; J. Halada leg.; OÖLM. - Mashonaland

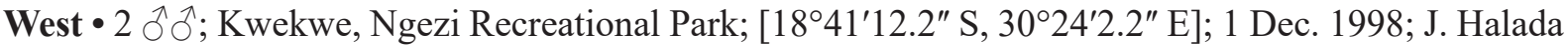
leg.; OÖLM • 1 क; Lomagundi [now Makonde District]; [16 $37^{\circ} 44^{\prime \prime}$ S, 3008'25" E]; 1 Mar. 1938; R.H.R. Stevenson leg.; RMNH. - Masvingo Province - 1 ô; Mushandike National Park, Masvingo; [2003'45" S, 3049'25" E]; 10 Dec. 1998; J. Halada leg.; OÖLM. - Matabeleland South • 1 \%; near Nalatale Ruins, 70 km SW of Gweru; [1952'57.5" S, 29³1'30.7" E]; Dec. 2011; J. Halada leg.; OÖLM.

\section{Description}

Female

Size. 22.5-27.9 mm.

CoLOR. Black except for the following, which are ferruginous: basal half of mandible, free clypeal margin and apical segment of metasoma. Forewing fuscous, with cyan-violet iridescence, hindwing slightly fuscous.

VeSTITURE. Appressed setae on clypeus and paraocular area silvery, on collar, scutum and propodeal enclosure black. Erect setae on clypeus, paraocular area, collar, scutum and propodeal enclosure black. Erect propodeal setae oriented posteriorly. Clypeus medially with broad vertical glabrous stripe. Scutellum densely and coarsely pubescent. 
STRUCTURE. Free clypeal margin with broad, gently notched, shovel-like process medially, not markedly stepped above. Clypeus with notable indentation in lower center and longitudinal carina above. Scutellum convex. Metanotum not raised, not bituberculate. $2^{\text {nd }}$ recurrent vein joins markedly proximal from interstitium between submarginal cells II and III. Propodeal enclosure without any notable ridges. Foretarsomere I 2.5-3.2 $\times$ length of antepenultimate spine. Petiole length 1.4-2.1 $\times$ its medial width.

\section{Male}

SizE. $15.2-24.1 \mathrm{~mm}$.

CoLOR. Black except for ferruginous stripe in center of mandible. Forewing fuscous, with cyan-violet iridescence, hindwing slightly fuscous.

VESTITURE. Appressed setae on clypeus, paraocular area, collar, posterolaterally on scutum and posteriorly of propodeal enclosure silvery, black on remainder of scutum and propodeal enclosure. Erect setae on clypeus, collar, scutum and propodeal enclosure black, uniformly black or intermixed with silvery ones on paraocular area and at posterior propodeal margin. Erect propodeal setae oriented posteriorly. Lower center of clypeus glabrous. Scutellum densely and coarsely pubescent. Metasomal sterna II-VII with increasingly dense fringes of black setae.

StRUCTURE. Free clypeal margin simple. Clypeus with notable carina in upper center. Scutellum convex. Metanotum not raised, not bituberculate. $2^{\text {nd }}$ recurrent vein joins markedly proximal from interstitium between submarginal cells II and III. Propodeal enclosure without any notable ridges. Posterior margin of metasomal tergum VII gently notched. Posterior margin of metasomal sternum VII simple, of metasomal sternum VIII concavely emarginate. Penis valvae without conspicuous modifications. Petiole length 2.6-2.8 $\times$ its medial width. Flagellomere V with narrow placoid covering its proximal four-fifths, and tapering medially or distally.

\section{Variation}

In males, the color of the appressed setae posterolaterally on the collar, scutum and propodeum as well as the erect setae at the posterior propodeal margin varies between silvery and black.

\section{Distribution}

Eastern to southeastern Africa.

\section{Remarks}

As mentioned above in the Remarks on S. incomptus, the subspecies proposed by Leclercq in 1955, $S$. incomptus anonymus, is actually a synonym of $S$. nigrohirtus, which resulted from confusing statements by Arnold (1928).

Sphex pulawskii sp. nov. urn:lsid:zoobank.org:act:0BD5A35B-90FD-4CB0-9340-394AE7E5D6FB

Figs 86-87, 91 (blue)

\section{Differential diagnosis}

Within the nigrohirtus group, females of $S$. pulawskii sp. nov. can be recognized through their silvery appressed setae posterolaterally on the collar and silvery appressed and erect setae at the posterior propodeal margin, in combination with the remaining erect propodeal setae being black (Fig. 86). Those of $S$. incomptus have most of the propodeum covered with silvery setae (Fig. 85), whereas female S. nigrohirtus have uniformly black mesosomal setae (Fig. 84). 
Males can be more difficult to identify, as some specimens of $S$. nigrohirtus share the combination of erect propodeal setae that are black on the enclosure and silvery at the posterior margin. The easiest characteristic to distinguish them is the absence of cyan iridescence on the wings of $S$. pulawskii sp. nov., being rich purple instead (Fig. 87).

\section{Etymology}

We dedicate this species to Dr Wojciech J. Pulawski, who collected some of the specimens and who is responsible for creating and maintaining the amazing Catalog of Sphecidae that has made our work much more manageable.

\section{Material examined}

\section{Holotype}

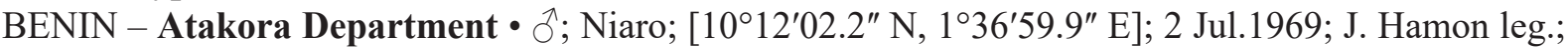
MNHN.

\section{Paratypes}

AFRICA • 1 क; 2 Feb. 1894; ZMB.

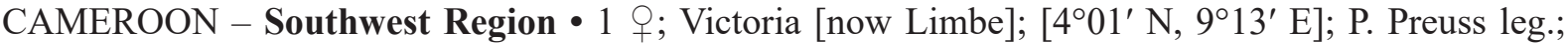

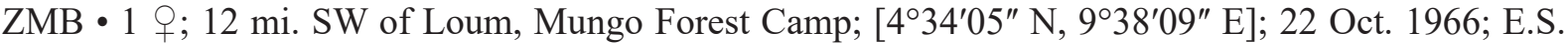
Ross and K. Lorenzen leg.; CAS.

DEMOCRATIC REPUBLIC OF THE CONGO - North Kivu • 1 \%; Mutsora; [0¹9'20" N, 2944'43" E]; 22 Apr. 1905; Hackars leg.; MRAC.

GHANA - Eastern Region • 2 우; Aburi; [550'52" N, 0¹0'28" W]; Dec. 1941; K.M. Guichard leg.; BMNH.

LIBERIA-Bong County • 1 \%; 10 mi. NE of Gbanka [Gbarnga]; [709'30" N, 9²7'07" W]; 11 Aug. 1966; E.S. Ross and K. Lorenzen leg.; CAS. - Gbarpolu County • 1 q; Kolobanu [Kolobani]; [ $7^{\circ} 12^{\prime} 07^{\prime \prime} \mathrm{N}$, $\left.9^{\circ} 52^{\prime} 38^{\prime \prime} \mathrm{W}\right]$; 19 Oct. 1926; J. Bequaert leg.; MRAC. - Lofa County • 1 क; 36 mi. S of Voinjama; [756'44" N, 9³2'10" W]; 13 Aug. 1966; E.S. Ross and K. Lorenzen leg.; CAS.

NIGERIA - Cross River State • 1 o; Obudu Cattle Ranch; [6 $6^{\circ} 22^{\prime}$ N, $9^{\circ} 22^{\prime}$ E]; 3 May 1973; J.T. Medler leg.; BMNH. - Edo State • 1 O$^{\text {; }}$; Benin; [620'17" N, 5³7'32" E]; 30 Jul. 195?; T. Davey leg.; BMNH. -

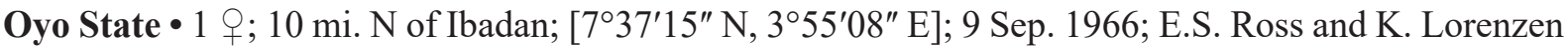

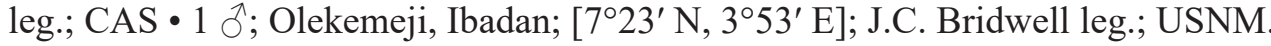

REPUBLIC OF CÔTE D'IVOIRE • 1 ' ; "Samplen"; 1910; A. Chevalier leg.; MNHN. - Lagunes

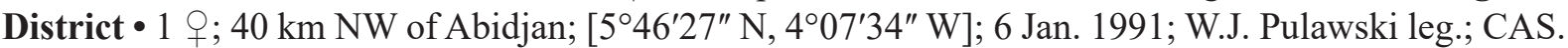

SIERRA LEONE • 1 \%; MNHN.

TOGO - Centrale Region - 1 o; Bismarckburg [near Yégué]; [8¹0'32.74" N, 041'09.42" E]; 20 Jul.-20 Sep. 1890; R. Büttner leg.; ZMB • 1 क; same collection data as for preceding; ZMB.

\section{Description}

\section{Female}

SIZE. 20.8-26.9 mm.

CoLOR. Black except for the following, which are ferruginous: basal half of mandible and free clypeal margin. Wings fuscous, with purple iridescence. 
Vestiture. Appressed setae on clypeus, paraocular area, collar, posterolaterally on scutum and at posterior propodeal margin silvery, on remainder of scutum and propodeal enclosure black. Erect setae on clypeus, frons, collar, scutum and propodeal enclosure black, on posterior margin of propodeum silvery. Central third of clypeus glabrous. Scutellum densely and coarsely pubescent.

STRUCTURE. Free clypeal margin with broad, gently notched, shovel-like process medially, not markedly stepped above. Clypeus with notable indentation in lower center and longitudinal carina above. Scutellum convex. Metanotum not raised, not markedly bituberculate. $2^{\text {nd }}$ recurrent vein joins markedly proximal from interstitium between submarginal cells II and III. Propodeal enclosure without any notable ridges. Foretarsomere I $2.8-2.9 \times$ length of antepenultimate spine. Petiole length $1.8-2.2 \times$ its medial width.

\section{Male}

SIZE. 20.6-21.7 mm.

COLOR. Black except for ferruginous stripe in center of mandible. Wings slightly fuscous, with markedly purple iridescence.

VeSTITURE. Appressed setae on clypeus, paraocular area, collar and posterior margin of propodeum silvery, on scutum and propodeal enclosure black. Erect setae on clypeus, paraocular area, collar and scutum black, on propodeal enclosure silvery intermixed with black, on posterior margin of propodeum silvery. Lower center of clypeus glabrous. Scutellum densely and coarsely pubescent. Metasomal sterna II-VI with increasingly dense fringes of black setae.

Structure. Free clypeal margin simple. Clypeus with longitudinal carina in upper center. Scutellum convex. Metanotum not raised, not bituberculate. $2^{\text {nd }}$ recurrent vein joins markedly proximal from interstitium between submarginal cells II and III. Propodeal enclosure without any notable ridges. Posterior margin of metasomal tergum VII slightly notched. Posterior margin of metasomal sternum VII simple, of metasomal sternum VIII concavely emarginate. Penis valvae without conspicuous modifications. Petiole length $2.2-2.7 \times$ its medial width. Flagellomere V with narrow placoid covering its proximal four-fifths and tapering medially or distally.

\section{Variation}

Unknown.

\section{Distribution}

Western Africa.

\section{Remarks}

After careful consideration, we came to the conclusion that while the distinguishing characters of this species are very subtle, its classification as a separate taxon is warranted nonetheless, the reasons for which are explained below.

Our studies show that the similar $S$. nigrohirtus is restricted to the southern and eastern parts of the African continent. However, S. pulawskii sp. nov. is found in western Africa, and it is characterized by having the appressed and erect setae on the propodeum, posterolaterally of the enclosure, colored silvery instead of black. Additionally, western females also have the appressed setae silvery posteriorly on the collar and posterolaterally on the scutum, whereas those from eastern and southern Africa have them black.

Males, however, are not so easy to define. The color of the appressed setae posterolaterally on collar, scutum and propodeum and the erect setae on the posterior propodeal margin varies between black and 
silvery in S. nigrohirtus even among specimens from the same locality. There is one character that, although difficult to objectively define, is obvious in western African males: the iridescence on the wings. In these, it is uniformly purple, resembling the color Byzantium, whereas the tone is more akin to Spanish violet and always contains shades of cyan in S. nigrohirtus.

\section{Species of the bohemanni group}

The bohemanni group contains the following six species and three subspecies: Sphex abbotti, S. abbotti nivarius subsp. nov., S. abyssinicus, S. bohemanni, S. pseudopraedator sp. nov., S. schoutedeni, S. schoutedeni malawicus subsp. nov., S. stadelmanni and $S$. stadelmanni rufus subsp. nov.

Members are characterized by posteriorly-oriented erect propodeal setae (Fig. 1), which they share with the species of the nigrohirtus group. The most prominent attributes that separate them are found on the female clypeus. In the bohemanni group, its appressed setae are brown or black (with a golden or brassy luster in some species, but not extending all the way to the clypeal center) (Figs 14-18), and the clypeus itself has an inconspicuous indentation at most and lacks a notable longitudinal carina. In contrast, females of the nigrohirtus group have entirely silvery appressed setae on the clypeus, a marked indentation in the lower center and a short but notable longitudinal carina in the upper center (Fig. 13); or their entire clypeus and paraocular area is covered densely with appressed rich golden setae in the case of $S$. ahasverus. Male specimens of the nigrohirtus group can be recognized through their smaller size and slender stature, as well as the more or less concavely emarginate posterior margin of sternum VIII, whereas males of the bohemanni group are built more stoutly and have the posterior half of sternum VIII usually entire and often shaped like a semicircle (Fig. 20). Notably, all male members of this group, except $S$. pseudopraedator sp. nov., are distinguishable to species level (but not to subspecies level) through a combination of the structure of sternum VII (Figs 19-20) and the genitalia, more specifically that of the pensivalvae (Figs 21-26). In the other groups, these have no easily discernible differences in the majority of members.

With the exception of S. pseudopraedator sp. nov., males of this group also have an extremely short (in the case of $S$. abyssinicus longer and acute), but very broad process emerging medially from the inner side of the free clypeal margin. Sphex ahasverus of the nigrohirtus group also possesses this feature. It is often very inconspicuous and not to be confused with the distinctly visible, less broad, tooth-like process present in males of the umtalicus group (Fig. 27).

Sphex abbotti abbotti W. Fox, 1891

Figs 15, 20, 88, 92 (red)

Sphex abbotti W. Fox, 1891: 42, $q$ (as Abbotii, incorrect original capitalization and spelling).

Sphex kilimandjaroensis Cameron, 1908: 262, §̂,, . Syn. nov.

Chlorion neavei Arnold, 1928: 370, ô, o +. Syn. nov.

\section{Differential diagnosis}

The males of this species possess a very recognizable feature, which is the markedly notched posterior margin of sternum VII (Fig. 20), a characteristic that they only share with S. bohemanni (Fig. 19) among species with uniformly black propodeal setae. Both can easily be distinguished by the fact that the notch is only about half as deep as the visible length of sternum VII in S. bohemanni, whereas it is significantly deeper in $S$. abbotti s. lat., so that the posteromedian part of sternum VII is almost or entirely covered by sternum VI. While $S$. abbotti nivarius subsp. nov. is identical to $S$. abbotti abbotti in these regards, the subspecies can be delimited based on having silvery erect propodeal setae (Fig. 89). 
Female specimens look very similar to those of $S$. stadelmanni stadelmanni, as both have uniformly black appressed clypeal and paraocular setae. However, the mandibles and free clypeal margin of S. stadelmanni stadelmanni are to a significant extent ferruginously-colored (Fig. 16), whereas they are nearly completely black in $S$. abbotti abbotti (Fig. 15). Furthermore, S. stadelmanni stadelmanni is known only from eastern South Africa and southwestern Mozambique (Fig. 99 [yellow]), while S. abbotti abbotti is found predominantly in east Africa (Fig. 92 [red]). Females of other species in the bohemanni group are characterized by a differently-colored luster of their appressed clypeal and paraocular setae (Figs 14, 17-18).

\section{Material examined}

Holotype

TANZANIA - Kilimanjaro Region • 9 ; Kilimanjaro; L. Abbott leg.; ANSP.

Other material

AFRICA・ 2 ふぇ, 1 ค; "Nyassa"; MNHN.

KENYA • 2 ơ ; near Cherangani Hills, 40 mi. E of Mt Elgon; [ $\left.1^{\circ} 15^{\prime} 05^{\prime \prime} \mathrm{N}, 35^{\circ} 26^{\prime} 33^{\prime \prime} \mathrm{E}\right]$; alt. $6200 \mathrm{ft}$; 15 Oct. 1924; C.R.S. Pitman leg.; BMNH. - Kiambu County 2 ठ $\jmath^{\jmath} ; 42$ km NW of Nairobi; $1^{\circ} 4.6^{\prime}$ S, 36³6.1' E; 3 Jun. 1949; W.J. Pulawski and J.S. Schweikert leg.; CAS • 1 đ', 1 ค; Kikuyu; 25 Feb. 1902; F. Thomas leg.; ZMB ・ 1 ○, 2 우; Kikuyu; 19 Apr. 1902; F. Thomas leg.; ZMB • 2 q ; ; Kikuyu, Kijabe; [056'42" S, 36³5'42" E]; Feb. 1912; Ch. Alluaud and Jeannel leg.; MNHN. - Kisii County • ${ }^{\wedge}$; Kisii District, S of Kavirondo; [ $0^{\circ} 40^{\prime} \mathrm{S}, 34^{\circ} 45^{\prime}$ E]; 9-12 May 1911; BMNH. - Laikipia County • 2 + $q$;

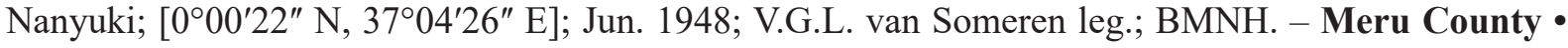
$1 \mathrm{o}^{\top}$; Meru; [002'46" N, 37³9'21" E]; Jul. 1943; V.G.L. van Someren leg.; BMNH. - Nairobi County •

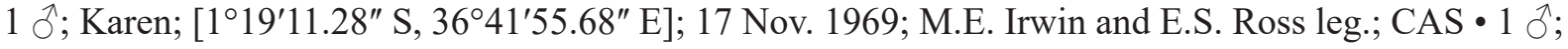

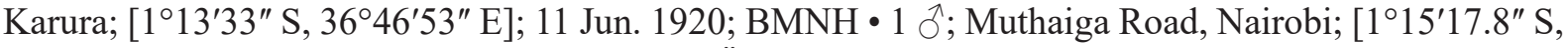
36²9'54.3" E]; 4 Apr. 2007; M. Halada leg.; OÖLM • 1 +; Nairobi; [1 ${ }^{\circ} 17^{\prime}$ S, 36²49' E]; Nov. 1925; G. Babault leg.; MNHN • 1 đ̃, 1 क ; same locality as for preceding; Dec. 1909; Ch. Alluaud leg.; MNHN • $1 \hat{\delta}$; same locality as for preceding; F.J. Jackson leg.; BMNH. - Nakuru County 1 o ; Naivasha; [043' S, 36²6' E]; Sep. 1906; Maurice de Rothschild leg.; MNHN. - Narok County • 1 क; Ngare Narok, Masai Reserve; [1 ${ }^{\circ} 02^{\prime} 40^{\prime \prime}$ S, 36 $6^{\circ} 09^{\prime} 47^{\prime \prime}$ E]; 21 Dec. 1913; A.O. Luckman leg.; BMNH. - Nyeri County • 1 क; Nyeri; [0²5'12" S, 36 $57^{\prime}$ E]; Dec. 1948; V.G.L. van Someren leg.; BMNH. - TaitaTaveta County • 1 ; Chawia Forest; 3.47908 ${ }^{\circ}$ S, 38.34162 ${ }^{\circ}$ E; 26 Dec. 2011-9 Jan. 2012; R. Copeland

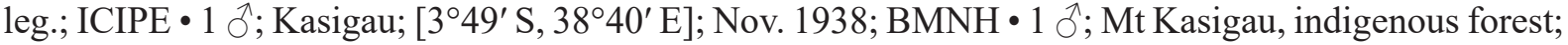
3.82700 ${ }^{\circ}$ S, 38.64875 ${ }^{\circ}$ E; $16-30$ Nov. 2011; R. Copeland leg.; THD-032-ICIPE; GenBank CO1 gene: MW538552; GenBank LWR gene: MW582289; ICIPE • 1 đ̊; Mt Mbololo; 5 May 1979; R. Murphy leg.; BMNH 1 ते; same locality as for preceding; Oct. 1938; V.G.L. van Someren leg.; BMNH •

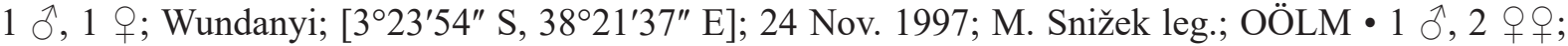
same locality as for preceding; 18-22 Mar. 1997; Ma. Halada leg.; OÖLM • 2 ô $\hat{\sigma}, 1$ O; same collection data as for preceding but 5-10 Apr. 1997; OÖLM • 2 ठో ; same locality as for preceding; 19-21 Nov. 1996; Mi. Halada leg.; OÖLM.

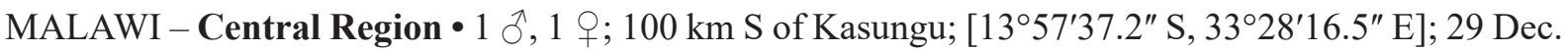

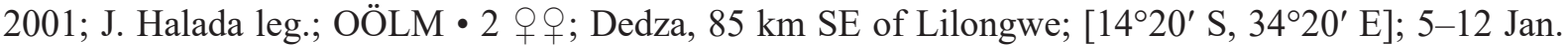

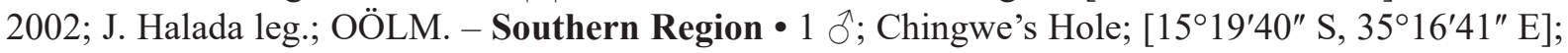
2 Nov. 1970; Schulter leg.; AMG • 1 q; Mlanje; [1601'53" S, 35³0' E]; 15 Jan. 1913; S.A. Neave leg.;

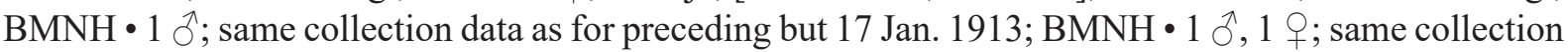
data as for preceding but 22 Jan. 1913; BMNH 1 1 ${ }^{\Uparrow}$, holotype or syntype of Chlorion neavei Arnold, 1928; same collection data as for preceding but 14 Mar. 1913; BMNH • 1 $\delta^{\lambda}$; same collection data as for preceding but 29 Dec. 1913; BMNH 1 ô, 1 q; same collection data as for preceding but 2 Jan. 1914; 


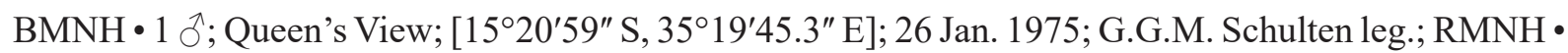

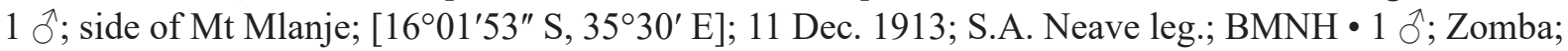

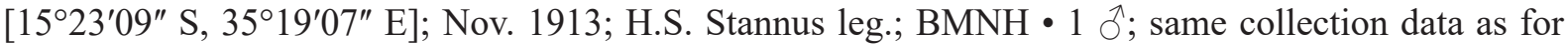
preceding but Jan. 1914; BMNH.

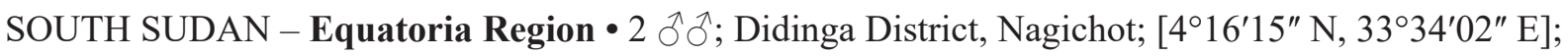
alt. 6700 ft; Dec. 1925-Jan. 1926; G.D.H. Carpenter leg.; BMNH.

TANZANIA - Arusha Region • 1 đo; Ngarenanyuki, Meru lowland; Jan.; Y. Sjöstedt leg.; NRS. - Kilimanjaro Region - 1 क; Kilimanjaro; W.L. Abbott leg.; USNM -1 , lectotype of Sphex kilimandjaroensis Cameron, 1908 (designated here); Kilimanjaro; 30 Oct. [1905?]; Y. Sjöstedt leg.; NRS •

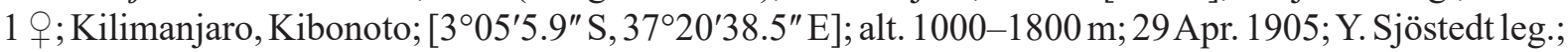
NRS 1 \% , paralectotype of Sphex kilimandjaroensis; same locality as for preceding; alt. 1300-1900 m; 26 Apr. 1905; Y. Sjöstedt leg.; BMNH 1 ô, paralectotype of Sphex kilimandjaroensis; same collection data as for preceding but 4 May 1905; BMNH $\bullet 1$, paralectotype of Sphex kilimandjaroensis; same collection data as for preceding but 9 May $1905 ; \mathrm{BMNH} \bullet 1 \hat{\jmath}, 1 \mathrm{q} ;$ same collection data as for preceding but 11 May 1905; NRS 1 ภे, paralectotype of Sphex kilimandjaroensis; Kilimanjaro, Kibonoto Kulturzone;

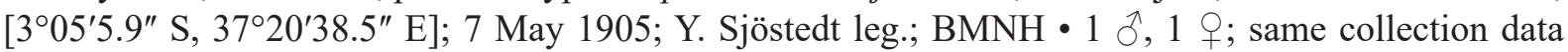
as for preceding but 12 May 1905; NRS $\bullet 1 \hat{\partial}, 1$ \%; same collection data as for preceding but 15 May

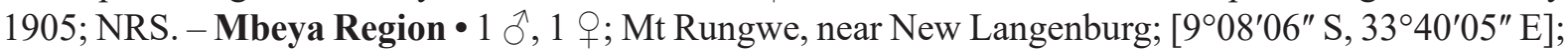
18-21 Nov. 1910; S.A. Neave leg.; BMNH・1 9 ; Usangu Dist.; 29 Nov.-15 Dec. 1910; S.A. Neave leg.;

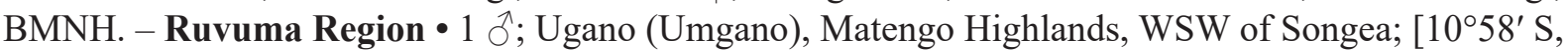
345'ㄹ' E]; alt. 1500-1700m; 23 Nov. 1937; F. Zimmer leg.; NHMW.

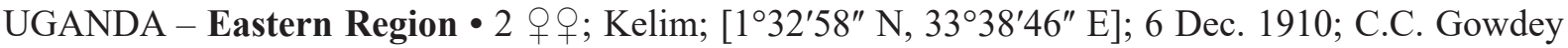

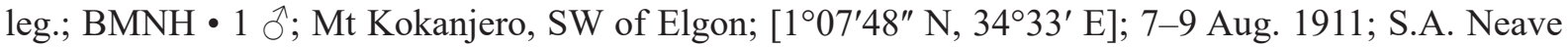
leg.; BMNH.

ZIMBABWE • 1 đ;; 10 Nov. 1938; R.H.R. Stevenson leg.; AMG. - Bulawayo • 1 §;; Bulawayo, Matobo;

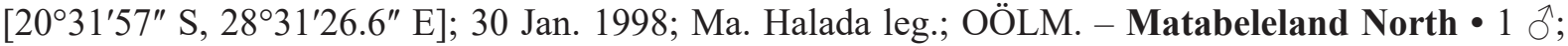

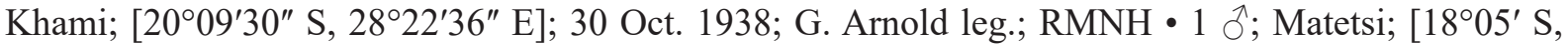
$26^{\circ} 07^{\prime}$ E]; Dec. 1933; R.H.R. Stevenson leg.; BMNH.

\section{Description}

Female

SizE. $26.8-29.5 \mathrm{~mm}$.

CoLOR. Black except for ferruginous stripe in center of mandible. Wings uniformly fuscous, with cyanpurple iridescence.

VESTITURE. Appressed and erect setae on clypeus, paraocular area, collar, scutum and propodeal enclosure black. Erect propodeal setae oriented posteriorly. Lower center of clypeus glabrous. Scutellum densely and coarsely pubescent.

STRUCTURE. Free clypeal margin medially with two broad, indistinct processes, stepped above. Clypeus with indentation in lower center, longitudinal carina in upper center barely noticeable. Scutellum convex. Metanotum not raised, not notably bituberculate. $2^{\text {nd }}$ recurrent vein joins markedly proximal from interstitium between submarginal cells II and III. Propodeal enclosure without any notable ridges, very indistinctly rugose in anterior third. Foretarsomere I $2.7-3.35 \times$ length of antepenultimate spine. Petiole length $1.6-1.8 \times$ its medial width. 
Male

Size. $22.5-32.2 \mathrm{~mm}$.

COLOR. Black except for the following, which are ferruginous: stripe in center of mandible, tergum VII and sterna VII-VIII. Wings slightly fuscous, with cyan-purple iridescence.

VeSTITURE. Appressed setae on clypeus and paraocular area brassy, on collar, scutum and propodeal enclosure black. Erect setae on clypeus, paraocular area, collar, scutum and propodeal enclosure black. Erect propodeal setae oriented posteriorly. Free clypeal margin glabrous. Scutellum densely and coarsely pubescent. Metasomal sterna II-VII with increasingly dense fringes of black setae.

StRUCTURE. Free clypeal margin simple. Scutellum convex. Metanotum not raised, not bituberculate. $2^{\text {nd }}$ recurrent vein joins markedly proximal from interstitium between submarginal cells II and III. Propodeal enclosure without any notable ridges. Posterior margin of metasomal tergum VII convex. Posterior margin of metasomal sternum VII deeply incised, of metasomal sternum semicircular. Penis valvae dorsally open, their outer margins anteriorly and inner margins posteriorly raised into broad lobes. Petiole length 1.5-1.8 $\times$ its medial width. Flagellomeres IV-VI with broad placoids covering their entire length.

\section{Variation}

Unknown.

\section{Distribution}

Eastern Africa.

\section{Remarks}

Our studies showed that the synonymisation of $S$. kilimandjaroensis by Turner (1918) and Berland (1927) with S. bohemanni was erroneous. The holotype of S. bohemanni is unambiguously characterized by a considerably less deeply incised posterior margin of sternum VII, as well as more narrow placoids on flagellomeres IV-VI.

Photographs of the holotype of $S$. abbotti have been examined. The combination of uniformly black setae on the head and thorax, a black clypeal margin and only a few red spots on the mandibles are very good indicators that this species is identical to $S$. kilimandjaroensis and $S$. neavei, especially in conjunction with the locality data. Thus, $S$. abbotti should become the valid name.

One female from the type series of $S$. kilimandjaroensis in the BMNH is not conspecific with the remainder of the specimens from the type series, but actually a member of $S$. nigrohirtus. Thus, its locality data are included in the list of material for that species instead of here.

Sphex abbotti nivarius subsp. nov. urn:1sid:zoobank.org:act:CBE2E9E4-17B4-46AA-8751-D8D095806598

Figs 14, 89, 92 (blue)

\section{Differential diagnosis}

Having a deep incision on the posterior margin of sternum VIII, males of this subspecies are wellcharacterized and can only be confused with those of $S$. abbotti abbotti (Fig. 20). The most notable difference between the two taxa is that male $S$. abbotti nivarius subsp. nov. have the erect (and in some cases additionally the appressed) propodeal setae silvery (Fig. 89) instead of black (Fig. 88). 
Females are more difficult to distinguish and greatly resemble those of $S$. schoutedeni schoutedeni, with which they share the silvery erect propodeal setae. Because their geographical distribution is roughly the same, assigning the females to the males was quite difficult. The only trait that separates them and simultaneously coincides with that of the respective male is the color of the appressed propodeal setae, which is black in S. schoutedeni schoutedeni and silvery in some females of S. abbotti nivarius subsp. nov., though it is black in others. The most reliable way to identify females with uniformly black appressed propodeal setae is the ratio of petiole length to width, which is around 2.0 in $S$. abbotti nivarius subsp. nov. and smaller than 1.7 in S. schoutedeni schoutedeni. Despite the similarities in the respective females, the male genitalia and sterna leave no doubt that $S$. schoutedeni and $S$. abbotti are clearly two definite species.

\section{Etymology}

'Nivarius' is a Latin adjective meaning 'snowy', which refers to the silvery-white setae on the propodeum that distinguish this subspecies.

\section{Material examined}

\section{Holotype}

ZAMBIA - North-Western Province - ${ }^{\top}$; E of Mufumbwe, NW of Kasempa; [1322'37" S, 2520'52.1" E]; 15 Nov. 2005; M. Snižek leg.; THD-016-OOLM; GenBank CO1 gene: MW538559; OÖLM.

\section{Paratypes}

ANGOLA - Huíla Province 1 9 ; 16 mi. W of Paiva Couceiro; [1449' S, 14³3’ E]; 9 Dec. 1966; E.S. Ross and K. Lorenzen leg.; CAS.

DEMOCRATIC REPUBLIC OF THE CONGO - Bas-Uele • 1 +; Bambesa; [ $\left.3^{\circ} 26^{\prime} 44.2^{\prime \prime} \mathrm{N}, 25^{\circ} 41^{\prime} 27^{\prime \prime} \mathrm{E}\right]$; 4 Oct. 1937; J. Vrydagh leg.; IRSN. - Haut-Katanga - 1 q; Elisabethville [now Lubumbashi];

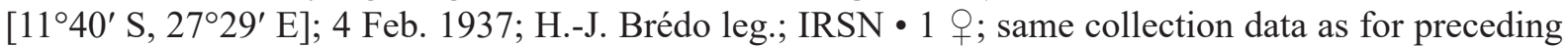
but 26 May 1937; IRSN • 1 क; same collection data as for preceding but 24 Dec. 1937; IRSN • 1 q; same locality as for preceding; Apr. 1928; M. Bequaert leg.; MRAC $\bullet 1$ i ; same locality as for preceding;

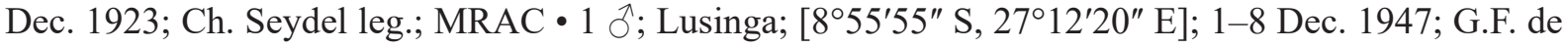

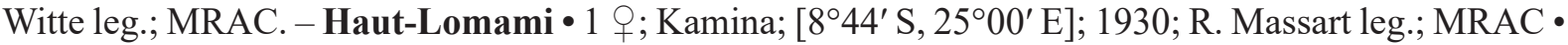

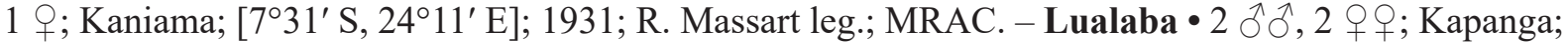

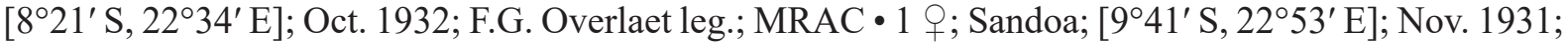
F.G. Overlaet leg.; MRAC.

ZAMBIA - North-Western Province $\bullet 2{ }^{\lambda} \partial^{\lambda}, 1$ क; $150 \mathrm{~km} \mathrm{SW}$ of Solwezi; $13^{\circ} 02^{\prime} \mathrm{S}, 25^{\circ} 45^{\prime} \mathrm{E}$; 15 Nov. 2005; M. Halada leg.; OÖLM • 1 q; $30 \mathrm{~km} \mathrm{E} \mathrm{of} \mathrm{Solwezi;} 12^{\circ} 21^{\prime} \mathrm{S}, 2^{\circ} 01^{\prime} \mathrm{E} ; 8$ Nov. 2005;

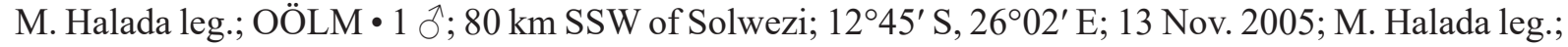
OÖLM • 1 O; Solwezi, 90 km E of Chisasa; [12¹1' S, 26²4' E]; 9 Sep. 2005; M. Snižek leg.; OÖLM • 1 ; near Solwezi; [12¹1' S, 26²4' E]; 1-3 Dec. 2002; J. Halada leg.; OÖLM.

\section{Description}

Female

SizE. $23.5-31.6 \mathrm{~mm}$.

COLOR. Black except for basal half of mandible and free clypeal margin, which are ferruginous. Wings uniformly fuscous, with violet-bluish iridescence.

VESTITURE. Appressed setae on clypeus silvery-golden, black towards center, on paraocular area silvery, on collar and scutum black, on propodeal enclosure black, near posterior margin silvery or black. Erect setae on clypeus, paraocular area, collar and scutum black, on propodeal enclosure silvery. Erect 
propodeal setae oriented posteriorly. Lower center of clypeus glabrous. Scutellum densely and coarsely pubescent.

STRUCTURE. Free clypeal margin medially with two broad, indistinct processes, not stepped above. Clypeus with indentation in lower center, without longitudinal carina. Scutellum convex. Metanotum not raised, not notably bituberculate. $2^{\text {nd }}$ recurrent vein joins markedly proximal from interstitium between submarginal cells II and III. Propodeal enclosure without any notable ridges. Foretarsomere I 2.8-3.3× length of antepenultimate spine. Petiole length $1.8-2.1 \times$ its medial width.

\section{Male}

Size. $25.5-29.7 \mathrm{~mm}$.

COLOR. Black except for the following, which are ferruginous: basal half of mandible, tergum VII and sterna VII-VIII. Wings slightly infuscate, with violet-bluish iridescence.

VeSTITURE. Appressed setae on clypeus and paraocular area brassy, anteriorly on collar, on scutum predominantly and anteriorly on propodeal enclosure black, posterolaterally on scutum and posteriorly on propodeum silvery. Erect setae on clypeus, paraocular area, collar and scutum black, on propodeal enclosure silvery. Erect propodeal setae oriented posteriorly. Free clypeal margin glabrous. Scutellum densely and coarsely pubescent. Metasomal sterna II-VII with increasingly dense fringes of black setae.

STRUCTURE. Free clypeal margin medially with very indistinct process emerging from inner side. Scutellum convex. Metanotum not raised, not bituberculate. $2^{\text {nd }}$ recurrent vein joins markedly proximal from interstitium between submarginal cells II and III. Propodeal enclosure without any notable ridges Posterior margin of metasomal tergum VII convex. Posterior margin of metasomal sternum VII deeply incised, of metasomal sternum VIII semicircular. Penis valvae fused, apical third dorsally open, with several anteriorly-oriented fin-like processes. Petiole length 1.7-2.0 $\times$ its medial width. Flagellomeres IV-VI with broad placoids covering their entire length.

\section{Variation}

Males can have the appressed facial setae pale silvery instead of brassy, or the appressed propodeal setae uniformly silvery instead of predominantly black.

\section{Distribution}

Central Africa.

Sphex abyssinicus (Arnold, 1928)

Figs 17, 25-26, 92 (yellow)

Chlorion abyssinicum Arnold, 1928: 372, ð̂.

\section{Differential diagnosis}

The male of this species can easily be identified through its uniquely rectangular-shaped eighth sternum (Fig. 25) and enlarged valviceps (Fig. 26). Females have been undescribed until now, but we were able to tentatively assign four female specimens to $S$. abyssinicus based on morphological considerations and geographical distribution. As is the case with many female members of the bohemanni group, they are hard to distinguish from closely related species, but the color of the luster of the appressed facial setae is a useful diagnostic. In this species, it is ferruginous, as well as golden laterally on the clypeus and the paraocular area (Fig. 17), which separates it from most others. Females of S. abbotti abbotti can 
look very similar (Fig. 15), but their facial setae lack a golden shimmer, whereas those of S. schoutedeni malawicus subsp. nov. possess it (Fig. 18), but are not known to occur north of Zambia.

\section{Material examined}

Holotype

ETHIOPIA • ô; 1911; R.E. Turner leg.; BMNH.

\section{Paratype}

ETHIOPIA $\bullet 1$; same collection data as for holotype; BMNH.

\section{Other material}

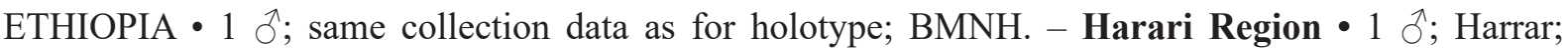

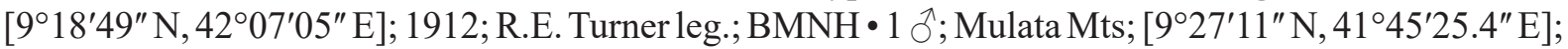
22 Oct. 1925; AMNH. - Oromia Region - 1 क; 23 km NW of Mena; 6³5' N, 3944' E; 28 May 2015; J. Halada leg.; OÖLM. - Southern Nations, Nationalities, and Peoples' Region • 1 q; Nera Dorze; 6 ${ }^{\circ} 10^{\prime} 8.4^{\prime \prime}$ N, 37³4'7.9" E; Apr. 2013; M. Ströhle leg.; OÖLM • 1 + ; same collection data as for preceding; THD-033-OOLM; GenBank LWR gene: MW582276; OÖLM.

\section{Description}

\section{Female}

SIZE. 24.5-30.2 mm.

CoLOR. Black, except for ferruginous stripe in center of mandible. Wings uniformly fuscous, with turquoise luster.

VeSTITURE. Appressed setae on clypeus, paraocular area, collar, scutum and propodeal enclosure black, on clypeus and paraocular area with ferruginous-golden luster. Erect setae on clypeus, paraocular area, collar, scutum and propodeal enclosure black. Erect propodeal setae oriented posteriorly. Lower center of clypeus glabrous. Scutellum densely and coarsely pubescent.

STRUCTURE. Free clypeal margin medially with two broad, indistinct processes, stepped above. Clypeus with indentation in lower center, without longitudinal carina. Scutellum convex. Metanotum not raised, not notably bituberculate. $2^{\text {nd }}$ recurrent vein joins markedly proximal from interstitium between submarginal cells II and III. Propodeal enclosure without any notable ridges. Foretarsomere I $2.8-2 \times$ length of antepenultimate spine. Petiole length $1.4-1.7 \times$ its medial width.

\section{Male}

SizE. $30.9 \mathrm{~mm}$.

CoLOR. Black except for basal half of mandible, which is ferruginous. Wings slightly fuscous, with violet iridescence.

VeSTITURE. Appressed setae on clypeus and paraocular area brassy, on collar, scutum and propodeal enclosure black. Erect setae on clypeus, paraocular area, collar, scutum and propodeal enclosure black. Erect propodeal setae oriented posteriorly. Lower center of clypeus glabrous. Scutellum densely and coarsely pubescent. Metasomal sterna II-VII with increasingly dense fringes of black setae.

STRUCTURE. Free clypeal margin medially with short, broad process emanating from inferior part. Scutellum convex. Metanotum slightly raised, not bituberculate. $2^{\text {nd }}$ recurrent vein joins markedly proximal from interstitium between submarginal cells II and III. Propodeal enclosure without any notable ridges. Posterior margin of metasomal tergum VII convex. Posterior margin of metasomal sternum VII 
simple, of metasomal sternum VIII concavely emarginate and broadly truncated apically. Penis valvae fused, inner margin flattened and dorsally markedly raised, outer margin bulging near apex. Petiole length 1.3-1.4 $\times$ its medial width. Flagellomeres IV-VI with broad placoids covering their entire length.

\section{Variation}

Unknown.

\section{Distribution}

Ethiopia.

Sphex bohemanni Dahlbom, 1845

Figs 19, 90, 92 (purple)

Sphex bohemanni Dahlbom, 1845: 436, §ิ.

Sphex transvaalensis Cameron, 1910: 140, ${ }_{0}$. Synonymized with Chlorion bohemanni by R. Turner 1918: 361.

\section{Differential diagnosis}

Males of this species (Fig. 90) can easily be recognized through their distinctly notched posterior margin of sternum VII (Fig. 19). They share this character with those of S. abbotti s. lat. (Fig. 20), but can be distinguished based on the depth of the incision. In $S$. abbotti, the incision is acute-angled and covers most or all of the visible part of the sternum, whereas it is obtuse-angled in $S$. bohemanni and reaches only approximately the halfway point of the visible sternum.

\section{Material examined}

\section{Holotype}

SOUTH AFRICA - KwaZulu-Natal • O; $^{\text {; }}$ Port Natal [now Durban]; NRS.

\section{Other material}

AFRICA・ 2 ふぇં; ZMB.

SOUTH AFRICA - Gauteng 1 ô, holotype or syntype of Sphex transvaalensis Cameron, 1910;

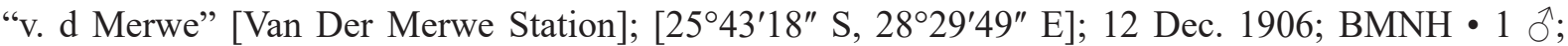

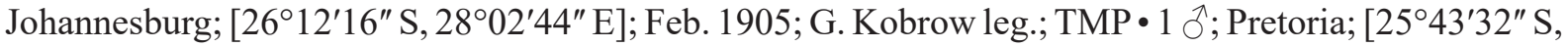

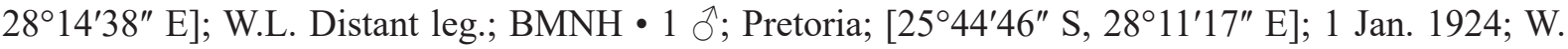

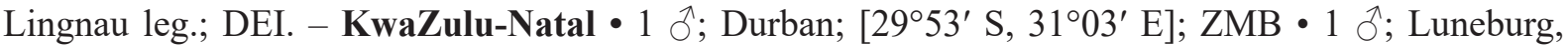

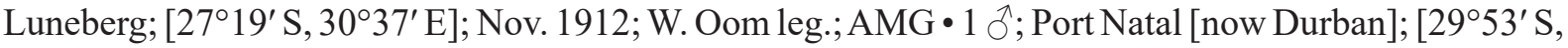

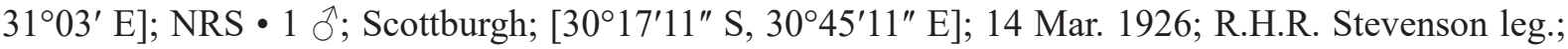
$\mathrm{BMNH}$

\section{Description}

Female

Unknown.

\section{Male}

Size. 27.3-30.6 mm.

COLOR. Black except for basal half of mandible, which is ferruginous. Wings fuscous, forewing with violet iridescence. 
VeSTITURE. Appressed setae on clypeus and paraocular area silvery, on collar, scutum and propodeal enclosure black. Erect setae on clypeus, paraocular area, collar, scutum and propodeal enclosure black, at posterior margin of propodeum black or silvery. Erect propodeal setae oriented posteriorly. Clypeus without glabrous spot. Scutellum densely and coarsely pubescent. Metasomal sterna III-VII with increasingly dense fringes of black setae.

StruCture. Free clypeal margin simple. Scutellum convex. Metanotum not raised, not bituberculate. $2^{\text {nd }}$ recurrent vein joins markedly proximal from interstitium between submarginal cells II and III. Propodeal enclosure without any notable ridges. Posterior margin of metasomal tergum VII convex. Posterior margin of metasomal sternum VII distinctly notched, of metasomal sternum VIII triangular. Petiole length 1.4-1.6 $\times$ its medial width. Flagellomeres IV-VI with narrow placoids, the first two covering proximal four-fifths of flagellomere length, last one of similar extent as first two, shorter or altogether absent.

\section{Variation}

Unknown.

\section{Distribution}

South Africa (Gauteng and KwaZulu-Natal provinces).

\section{Remarks}

While male specimens of $S$. bohemanni display a very conspicuous character for identifications as noted above, female specimens are treated as unknown, since they are presumably practically indistinguishable from those of several other African species in this group, namely $S$. abbotti abbotti and S. stadelmanni stadelmanni. They also occur in the same approximate geographic region as S. stadelmanni stadelmanni.

Sphex pseudopraedator $\mathrm{sp}$. nov. urn:1sid:zoobank.org:act:12625520-0846-455C-9F27-1DF41BBC53C8

Figs 1, 93, 98

\section{Differential diagnosis}

Sphex pseudopraedator sp. nov. (Fig. 93) can easily be distinguished from other members of the group through its yellow-tinged cellular wing area.

\section{Etymology}

Pseudo- is a prefix derived from the Greek adjective 'pseudếs' meaning 'false', 'pseudopraedator' thus refers to the fact that this species has been incorrectly identified in the past as the Oriental S. luteipennis Mocsáry, 1883, which is a synonym of $S$. praedator F. Smith, 1858.

\section{Material examined}

Holotype

ZAMBIA - Copperbelt Province • 9 ; $50 \mathrm{~km} \mathrm{~W}$ of Chingola; [12² $\left.25^{\prime} 06.3^{\prime \prime} \mathrm{S}, 2^{\circ} 23^{\prime} 50.9^{\prime \prime} \mathrm{E}\right] ; 1-2 \mathrm{Jan}$. 2003; J. Halada; OÖLM.

\section{Paratypes}

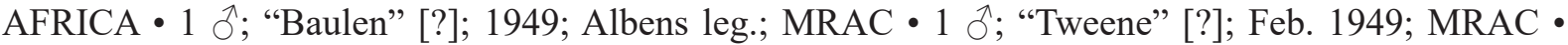
2 우; Cape of Good Hope; MNHN.

ANGOLA - Huíla Province • 1 q; 5 mi. NE of Negola; [1404'55" S, 14³2'52" E]; 25 Mar. 1972; BMNH. 


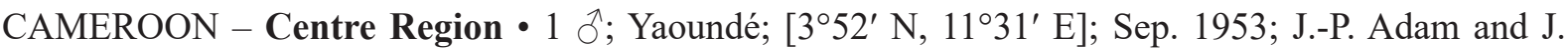
Rageau leg.; MNHN 1 ก, 1 \&; same collection data as for preceding; MNHN.

CENTRAL AFRICAN REPUBLIC - Kémo • 1 q; Sibut; [544'16" N, 1905'12" E]; MNHN.

DEMOCRATIC REPUBLIC OF THE CONGO • 1 \%; Katanga, N'Zenke; Mar. 1925; Ch. Seydel leg.;

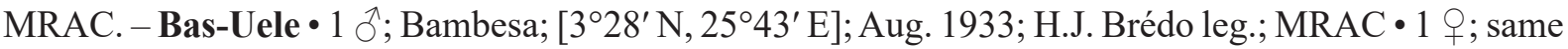
collection data as for preceding but 10 Sep. 1933; MRAC $\bullet 1$ \%; same collection data as for preceding but 15 Sep. 1933; MRAC • 1 ô, 2 q + ; same collection data as for preceding but 20 Sep. 1933; MRAC • 1 q; same collection data as for preceding but 25 Sep. 1933; MRAC $\bullet 1$; same collection data as for

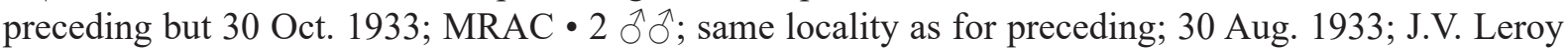
leg.; MRAC $\bullet 4$ 우; same collection data as for preceding but 10 Oct. 1933; MRAC • 1 क; same collection data as for preceding but Dec. 1934; MRAC 1 त; same locality as for preceding; Jun. 1937; J. Vrijdagh leg.; MRAC. - Haut-Katanga • 1 क; Elisabethville [now Lubumbashi]; [11 ${ }^{\circ} 40^{\prime} \mathrm{S}, 2^{\circ} 29^{\prime} \mathrm{E}$ ]; Oct. 1924; Ch. Seydel leg.; MRAC • 1 \%; same collection data as for preceding but 12 Jun. 1949; USNM •

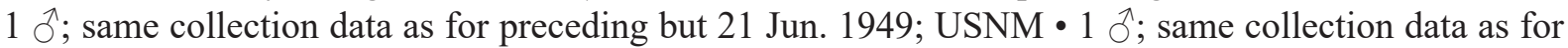
preceding but 5 Feb. 1953; MRAC. - Haut-Uele 1 of; Doruma; [ $4^{\circ} 43^{\prime}$ N, $27^{\circ} 41^{\prime}$ E]; 1934; R.P. De Graer leg.; MRAC. - Ituri Province • 1 \%; Mahagi-Niarembe; [2 $14^{\prime} 49^{\prime \prime} \mathrm{N}, 31^{\circ} 06^{\prime} 41^{\prime \prime}$ E]; Sep. 1935;

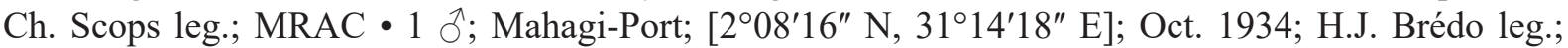
MRAC. - Kwango • 1 § ; Kimuanga; 27 Jan. 1930; Mevr. Bequaert leg.; MRAC. - Lualaba • 1 O ;

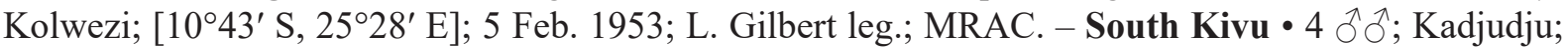
[209' S, 2854' E]; 1932; G. Babault leg.; MNHN.

KENYA - Embu County • 1 đ̊; Kirimiri Forest; $0.42563^{\circ}$ S, 37.54660 E; 28 Apr.-12 May 2011; R. Copeland leg.; ICIPE. - Kisii County 1 1 ${ }^{\top}$; Kisii District, S of Kavirondo; [0 $0^{\circ} 40^{\prime} \mathrm{S}, 34^{\circ} 45^{\prime}$ E]; 9-12 May 1911; S.A. Neave leg.; BMNH. - Trans-Nzoia County • 1 o; Kitale; $\left[1^{\circ} 01^{\prime} \mathrm{N}, 35^{\circ} 00^{\prime} \mathrm{E}\right]$; 1932-1933; C. Arambourg, P.-A. Chappuis and R. Jeannel leg.; MNHN.

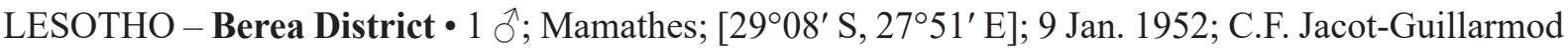
leg.; BMNH. - Leribe • 1 क ; 3 Jan. 1932; C. Jacot-Guillarmod leg.; TMP.

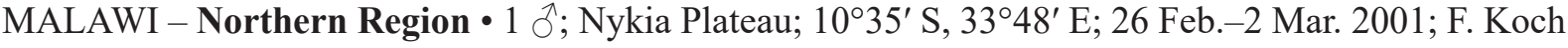

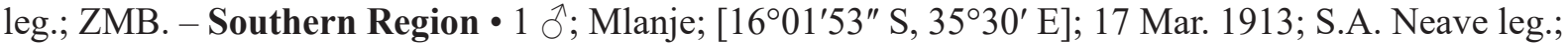

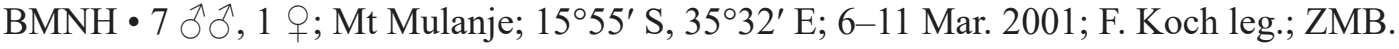

MOZAMBIQUE - Manica Province • 1 ớ; near Andrada, Valee du Revoué; [ $\left.18^{\circ} 53^{\prime} 41^{\prime \prime} \mathrm{S}, 32^{\circ} 51^{\prime} 16^{\prime \prime} \mathrm{E}\right]$; 1905; G. Vasse leg.; MNHN. - Manicaland • 1 q; Vumba; [1906' S, 3247’ E]; 20 Feb. 1931; P.A. Sheppard leg.; TMP.

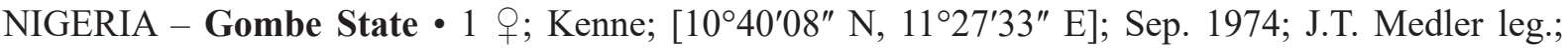

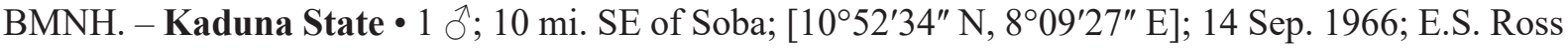
and K. Lorenzen leg.; CAS. - Taraba State 1 o ; Gashaka Gumti National Park, 30 km SE of Serti; $7^{\circ} 21^{\prime}$ N, 11³2' E; 24 Apr.-8 May 2011; J. Halada leg.; RMNH.

REPUBLIC OF CÔTE D'IVOIRE - Abidjan District • 2 q 9 ; Banco National Park; [ $\left.5^{\circ} 23^{\prime} \mathrm{N}, 4^{\circ} 03^{\prime} \mathrm{W}\right]$; R. Paulian and C. Delamare leg.; MNHN.

RWANDA - Western Province • 1 q; Ishwa, Lake Albert; [2²3’04" S, 2855'58” E]; Sep. 1935; H.J. Brédo leg.; MRAC. 


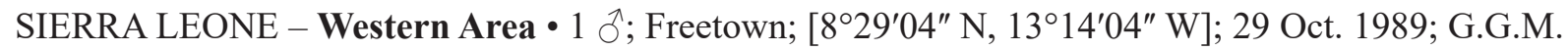

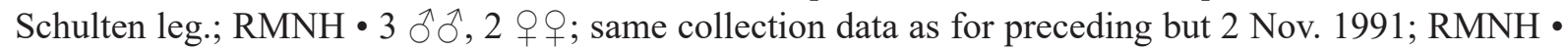

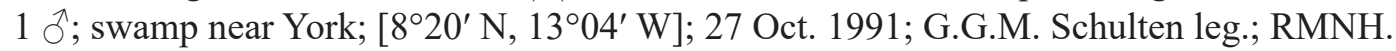

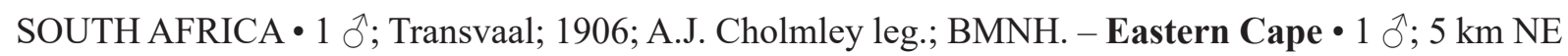
of Mount Frere, roadside; [3051'32" S, 2901'31.7" E]; 13 Feb. 1974; A.B. Gurney leg.; USNM • 1 o;

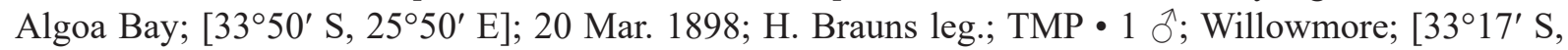
$23^{\circ} 29^{\prime}$ E]; Dec. 1919; H. Brauns leg.; TMP. - Gauteng - 2 ô $^{\lambda}$; E of Johannesburg; [26 $12^{\prime} 16^{\prime \prime}$ S,

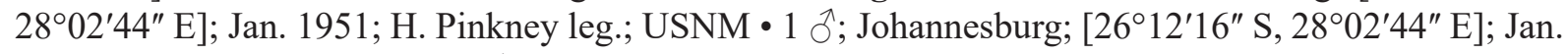

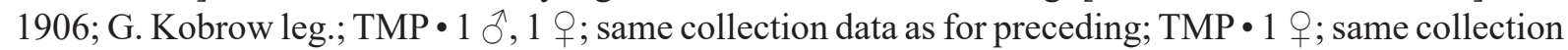

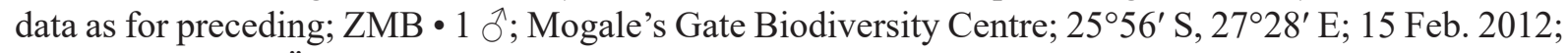
M. Ströhle leg.; OÖLM • 1 क; near Johannesburg; [26²1'16" S, $28^{\circ} 02^{\prime} 44^{\prime \prime}$ E]; 1906; A.J. Cholmley leg.;

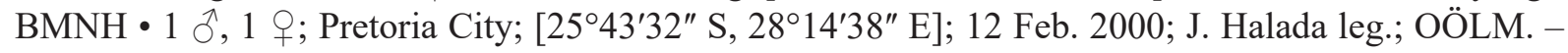

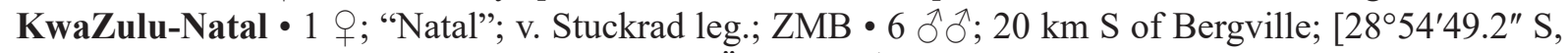
29²1'10.7" E]; 5 Feb. 2001; M. Snižek leg.; OÖLM • 1 đ'; 20 km W of Port Shepstone; [3046'50.9" S, $30^{\circ} 12^{\prime} 38^{\prime \prime}$ E]; 2 Feb. 2000; J. Halada leg.; OÖLM • 1 क; Camperdown; [2944' S, 30³2' E]; 10 Mar. 1908; G.F. Leigh leg.; TMP • 1 đ̊; Drakensberge, Royal Natal National Park; 2843' S, 2853' E; 9 Feb. 1994; F. Koch leg.; THD-000-ZMB; GenBank CO1 gene: MW538560; ZMB • 1 đ; same collection data as for preceding; THD-027-ZMB; GenBank LWR gene: MW582290; ZMB • 1 §ं; same collection data as for preceding; ZMB 1 क; Durban, Yellowwood Park; [28 $55^{\prime}$ S, 30 $30^{\circ} 6^{\prime}$ E]; 7 Mar. 1976; F.J. Herbst

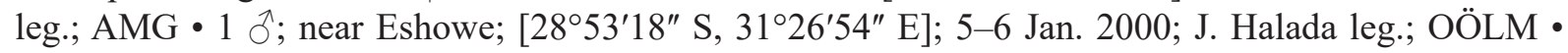

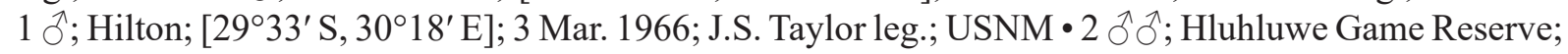
$28^{\circ} 02^{\prime} \mathrm{S}, 32^{\circ} 05^{\prime} \mathrm{E}$; $10 \mathrm{Feb} .1995$; F. Koch leg.; ZMB 11 \%; same collection data as for preceding but

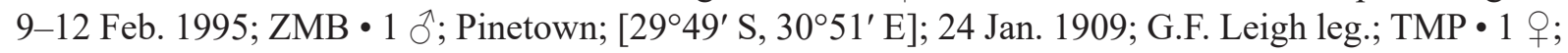

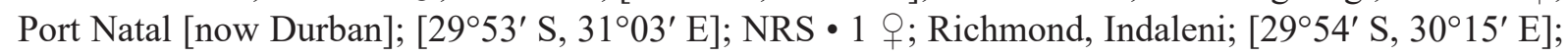
16 Jan. 1953; J.W. Hunt leg.; MRAC 11 ; same locality as for preceding; J.W. Hunt leg.; MRAC •

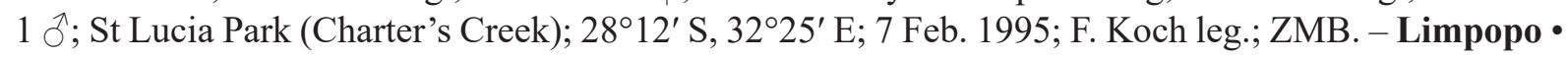
1 क; Lekgalameetse Nature Reserve; $24^{\circ} 12^{\prime}$ S, 30 30' $20^{\prime}$ E; 26 Mar.-1 Apr. 2001; F. Koch leg.; ZMB •

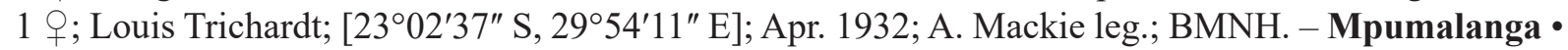

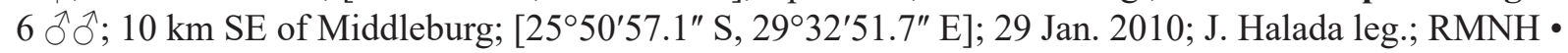

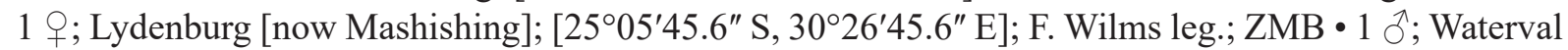

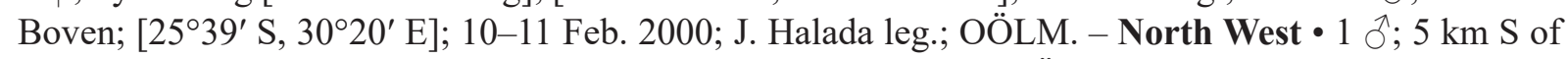

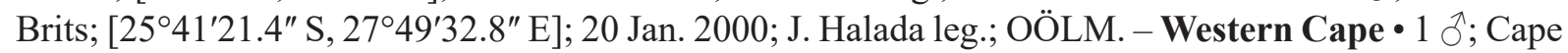
Town; [335' S, $18^{\circ} 25^{\prime}$ E]; Oct. 1949; S. Straw leg.; MRAC.

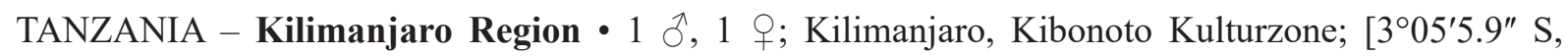
37²0'38.5" E]; 7 May 1905; Y. Sjöstedt leg.; NRS • 1 क; same collection data as for preceding but 12 May 1905; BMNH • 1 \%; same collection data as for preceding; NRS • 1 \%; same collection data as for

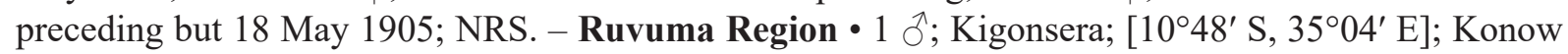
leg.; DEI.

TOGO - Centrale Region • 1 क; Bismarckburg [near Yégué]; [8¹0’32.74" N, 041'09.42" E]; 30 Jun.-3 Jul. 1893; L. Conradt leg.; ZMB.

UGANDA - Central Region • 1 क; Bweya; [0¹1'39" N, 32³2'59" E]; 22 May 1913; C.C. Gowdey leg.; BMNH • 1 q; Entebbe; [0 $0^{\circ} 03^{\prime} \mathrm{N}, 32^{\circ} 27^{\prime} 36^{\prime \prime}$ E]; 30 May 1913; C.C. Gowdey leg.; BMNH. -

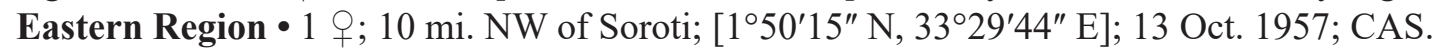

ZAMBIA - Copperbelt Province -1 ; same collection data as for holotype; OÖLM. - Lusaka

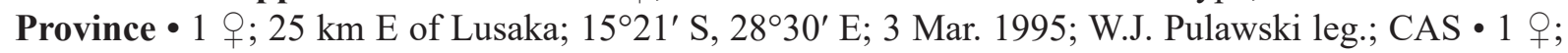


Lusaka International Airport; 15¹9' S, 28²7' E; 26 Mar. 1998; W.J. Pulawski leg.; CAS. - Northern

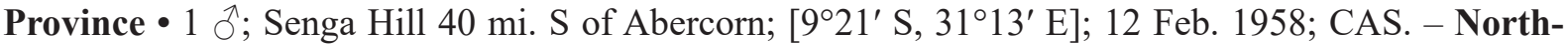

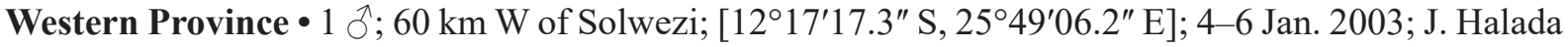

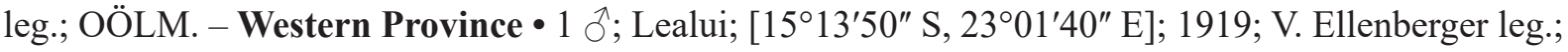
MNHN.

ZIMBABWE • 1 ภ, 1 क; Sanyati Valley; Sep.-Dec. 1925; R.H.R. Stevenson leg.; TMP • 1 q; Vumba

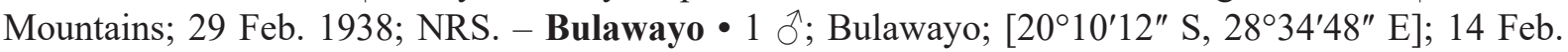
1924; R.H.R. Stevenson leg.; USNM. - Bulawayo Province • 1 đo; Feb. 1924; R.H.R. Stevenson leg.;

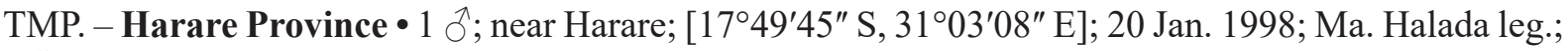

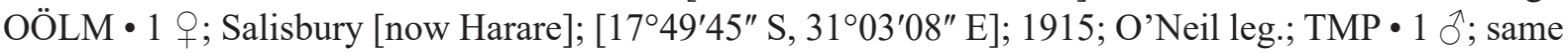
locality as for preceding; Feb. 1978; A. Watsham leg.; BMNH. - Manicaland • 1 ते; Mount Selinda;

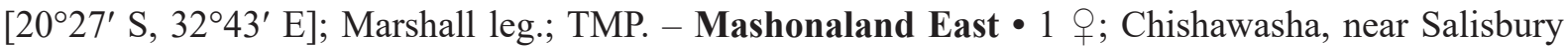

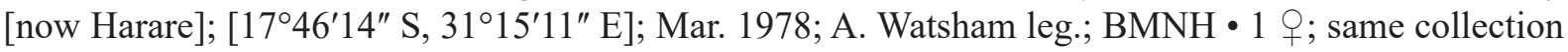
data as for preceding but Mar. 1982; BMNH.

\section{Description}

Female

SizE. 25.1-28.9 mm.

COLOR. Black except for ferruginous stripe in center of mandible. Cellular wing area yellowish, apical margin of forewing fuscous.

VeSTITURE. Appressed and erect setae on clypeus, paraocular area, collar and scutum black, appressed setae on propodeum black. Erect setae on propodeal enclosure black interspersed with golden ones, on posterior propodeal margin golden. Erect propodeal setae oriented posteriorly. Lower center of clypeus glabrous. Scutellum densely and coarsely pubescent.

STRUCTURE. Free clypeal margin with two notches near borders of approximate central third of clypeus. Free margin not stepped medially, clypeus with slight indentation in lower center, without carina. Scutellum convex. Metanotum not raised, not bituberculate. $2^{\text {nd }}$ recurrent vein joins slightly proximal from interstitium between submarginal cells II and III. Propodeal enclosure without any notable ridges. Foretarsomere I 3.1-3.5 $\times$ length of antepenultimate spine. Petiole length $2.2-2.4 \times$ its medial width.

\section{Male}

Size. 22.7-28.1 mm.

Color. Black except for ferruginous stripe in center of mandible. Cellular wing area of yellowish, apical margin of forewing fuscous.

VeSTITURE. Appressed setae on clypeus and paraocular area silvery, on collar, scutum and propodeal enclosure black. Erect setae on clypeus, paraocular area, collar, scutum and propodeal enclosure black, erect setae towards posterior margin of propodeum golden or silvery. Erect propodeal setae oriented posteriorly. Lower center of clypeus glabrous. Scutellum densely and coarsely pubescent. Metasomal sterna III-VII with increasingly dense fringes of black setae.

STRUCTURE. Free clypeal margin medially slightly emarginate. Scutellum convex. Metanotum not raised, not bituberculate. $2^{\text {nd }}$ recurrent vein joins markedly proximal from interstitium between submarginal cells II and III. Propodeal enclosure without any notable ridges. Posterior margin of metasomal tergum VII convex. Posterior margin of metasomal sternum VII simple, of metasomal sternum VIII very slightly concavely emarginate. Petiole length $2.3-2.7 \times$ its medial width. Flagellomeres IV-VI with broad placoids covering their entire length. 


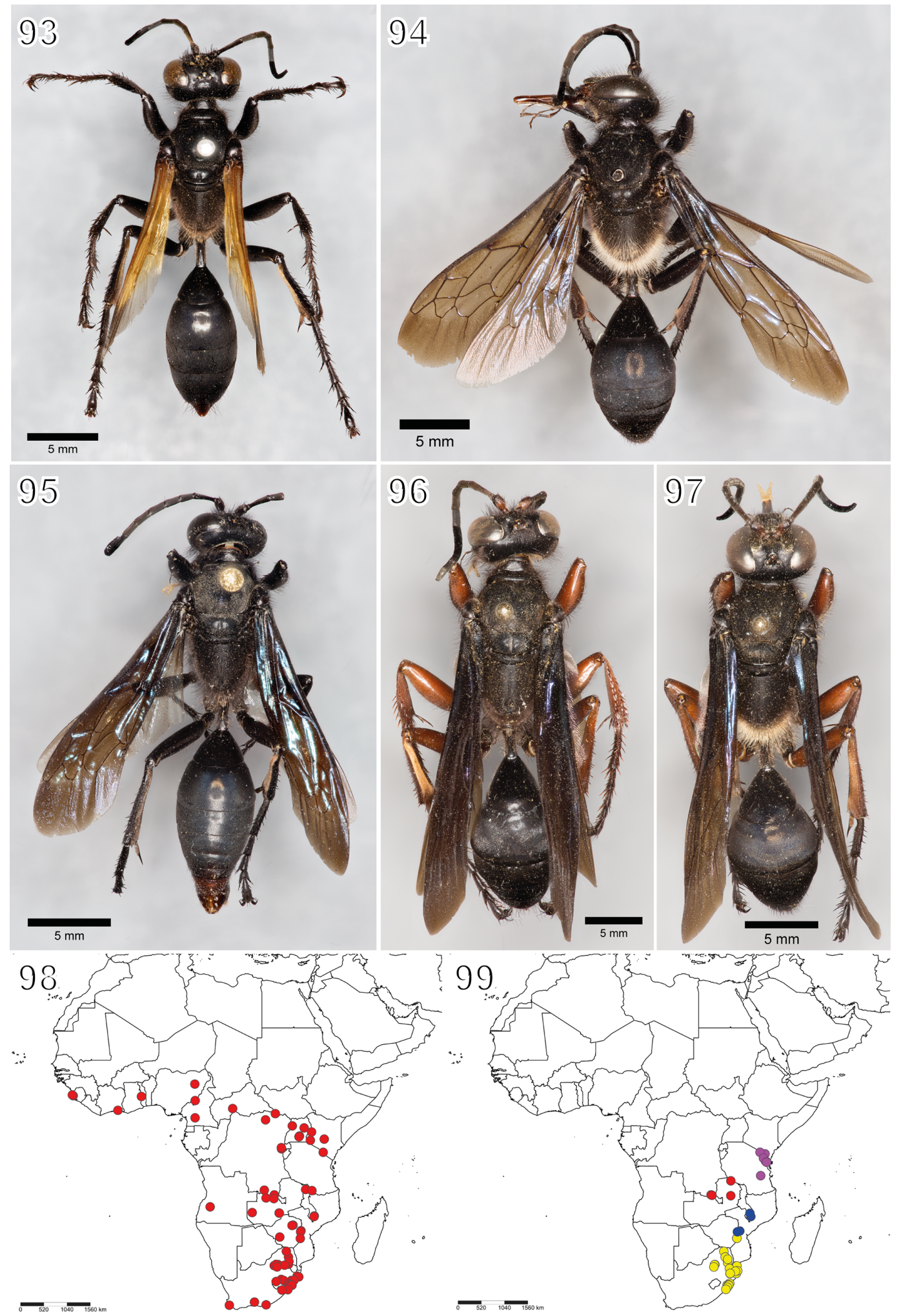

Figure 93-99. 93, 96. Habitus of $\uparrow .94-95$, 97. Habitus of $\partial$. 93. Sphex pseudopraedator sp. nov. 94. S. schoutedeni schoutedeni Kohl, 1913. 95. S. stadelmanni stadelmanni Kohl, 1895. 9697. S. stadelmanni rufus subsp. nov. 98. Geographic distribution of S. pseudopraedator sp. nov. 99. Geographic distribution of $S$. schoutedeni schoutedeni (red); S. schoutedeni malawicus subsp. nov. (blue); S. stadelmanni stadelmanni (yellow); S. stadelmanni rufus subsp. nov. (purple). 


\section{Variation}

In males, the color of the erect mesosomal setae ranges from almost uniformly black with only a few golden ones at the posterior propodeal margin to the erect propodeal setae being predominantly silverygolden with a few darker ones in between and the erect setae on the collar, scutum, scutellum and metanotum being partially or mostly silvery as well.

\section{Distribution}

Widespread in Africa.

\section{Remarks}

This species' female is similar to $S$. praedator, and its members have been repeatedly misidentified as S. luteipennis, a synonym of S. pradedator (Hensen 1991). However, as noted first by van der Vecht (1973), and later by Bohart \& Menke (1976) and Dollfuss (2008), the specimens from Africa must be of a different species. Our study of the holotype of $S$. praedator and several specimens showed that they can be easily distinguished: in S. pseudopraedator sp. nov., the erect propodeal setae are uniformly oriented posteriorly and mostly straight, while in S. praedator, they are perpendicular or oriented slightly anteriorly, and the tips are often curved towards the anterior. This can best be seen on the anterior third of the propodeum. Moreover, the males of both species are very conspicuously different in their wing color, with $S$. pradedator having the wings markedly fuscous with a greenish iridescence, whereas those of S. pseudopraedator sp. nov. have a similar coloration as in the female, with the cellular area yellowish.

\section{Sphex schoutedeni schoutedeni Kohl, 1913}

Figs 94, 99 (red)

Sphex schoutedeni Kohl, 1913: 205, ô.

\section{Differential eiagnosis}

Males of this species are easiest to recognize based on the structure of their posterior sterna. Lacking an incision on the posterior margin of sternum VII and having sternum VIII semicircular and simple, they resemble S. stadelmanni stadelmanni (Fig. 95). While that species has uniformly black erect setae at least on the propodeal enclosure, these setae are mostly silvery in S. schoutedeni schoutedeni (Fig. 94). Close examination of the valvicepes also reveals notable structural differences between these species (Figs 21-24). The subspecies $S$. schoutedeni malawicus subsp. nov. is very similar to the nominotypical form, but has the erect propodeal setae black, as in S. stadelmanni stadelmanni.

Females are much more difficult to identify. Due to the absence of visible sternal or genitalia characters, the color of their erect and appressed propodeal setae is one of the only useful diagnostics. Sphex abbotti nivarius subsp. nov. (Fig. 89) has silvery erect propodeal setae as well, and members of that somewhat variable subspecies which possess black appressed propodeal setae are almost indistinguishable. However, their petiole is around twice as long as it is wide, whereas that of female S. schoutedeni schoutedeni is at most $1.7 \times$ as long as wide.

\section{Material examined}

\section{Holotype}

ZAMBIA - Muchinga Province • $\lesssim$; Mpika; Jan. 1908; S.A. Neave leg.; MRAC.

\section{Other material}

DEMOCRATIC REPUBLIC OF THE CONGO - Haut-Katanga -1 ô; Elisabethville [now

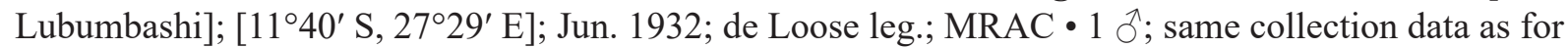

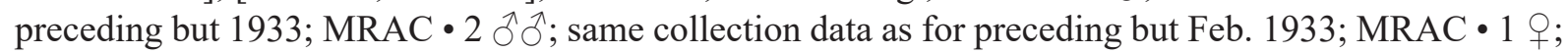


same collection data as for preceding but Apr. 1933; MRAC $\bullet 1$ त; same locality as for preceding; 2 Jan.

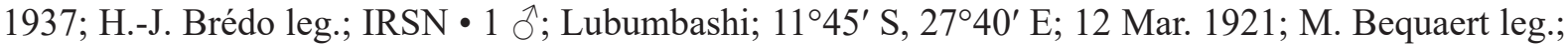
MRAC $\bullet 1$ § ; Kasepa, river; 1920; M. Bequaert leg.; MRAC.

ZAMBIA - Muchinga Province • 1 \&; "L. Chambezi V., Mpika distr.”; alt. 3900 ft; 19 May 1908; S.A.

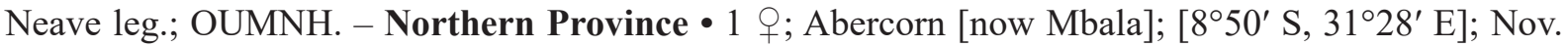
1943; IRSN • 1 §ं; same locality as for preceding; 14 Feb. 1951; F.O. Albrecht leg.; BMNH.

\section{Description}

Female

Size. 23.4-28.9 mm.

CoLOR. Black, except for basal half of mandible and free clypeal margin which are ferruginous. Wings uniformly fuscous, with purple-bluish iridescence.

VeSTITURE. Appressed setae on clypeus black, at lateral clypeal margin and on paraocular area brassy. Appressed setae on collar, scutum, propodeal enclosure and posterior propodeal margin black. Erect setae on clypeus, paraocular area, collar and scutum black, on propodeal enclosure and posterior propodeal margin silvery. Erect propodeal setae oriented posteriorly. Lower center of clypeus glabrous. Scutellum densely and coarsely pubescent.

STRUCTURE. Free clypeal margin medially with two broad, indistinct processes, stepped above. Clypeus with slight indentation in lower center, without carina. Scutellum convex. Metanotum not raised, not bituberculate. $2^{\text {nd }}$ recurrent vein joins markedly proximal from interstitium between submarginal cells II and III. Propodeal enclosure without any notable ridges. Foretarsomere I 2.6-2.9 $\times$ length of antepenultimate spine. Petiole length $1.5-1.7 \times$ its medial width.

\section{Male}

Size. 25.4-25.8 mm.

CoLOR. Black except for the following, which are ferruginous: basal half of mandible and free clypeal margin partially. Wings slightly infuscate, with violet iridescence.

VestiTURE. Appressed setae on clypeus and paraocular area brassy, on collar, scutum and propodeal enclosure black. Erect setae on clypeus, paraocular area, collar and scutum black, on propodeal enclosure brassy intermixed with black, on posterior margin of propodeum silvery. Erect propodeal setae oriented posteriorly. Clypeus with glabrous spot medially at free margin. Scutellum densely and coarsely pubescent. Metasomal sterna II-VII with increasingly dense fringes of black setae.

STRUCTURE. Free clypeal margin simple. Scutellum convex. Metanotum not raised, not bituberculate. $2^{\text {nd }}$ recurrent vein joins markedly proximal from interstitium between submarginal cells II and III. Propodeal enclosure without any notable ridges. Posterior margin of metasomal tergum VII convex. Posterior margin of metasomal sternum VII simple, of metasomal sternum VIII semicircular. Fused penis valvae laterally not widened, with notch, valviceps oriented perpendicularly. Petiole length $1.4-1.6 \times$ its medial width. Flagellomeres IV-VI with broad placoids covering their entire length.

\section{Variation}

In a male specimen from Angola, the appressed propodeal setae are silvery instead of black. Females often have the erect silvery setae on the propodeal enclosure interspersed with some black ones.

\section{Disribution}

Central Africa. 
Sphex schoutedeni malawicus subsp. nov.

urn:lsid:zoobank.org:act:59F1F204-D7F6-44E0-A5D8-11478E9D2A52

Figs 18, 23-24, 99 (blue)

\section{Differential diagnosis}

Males of this subspecies are most easily identifiable through the structure of their genitalia. Like S. schoutedeni schoutedeni, they have the fused penis valvae dorsally open, laterally not widened and with a notch (Figs 23-24). In the similar S. stadelmanni stadelmanni (Figs 21-22), the valvae are laterally widened, but lack a notch, and the valvicipites are curved posteroventrally and not perpendicularlyoriented as in S. schoutedeni s. lat. The males of the other species and subspecies of the bohemanni group all differ in having sternum VII or VIII modified in a conspicuous way (Figs 19-20, 25), whereas S. schoutedeni malawicus subsp. nov. has sternum VII without a notch and sternum VIII convex and entire. Finally, the nominotypical $S$. schoutedeni schoutedeni is distinguishable through having partially silvery propodeal setae (Fig. 94), while S. s. malawicus subsp. nov. has them black, with the exception of some erect golden setae at the posterior propodeal margin.

Females can be recognized based on the color of their clypeus and its vestiture. They, like all members of the bohemanni group, have the appressed setae black in the clypeal center, but the presence of golden appressed lateroclypeal and paraocular setae combined with a slightly ferruginous free clypeal margin is unique (Fig. 18).

\section{Etymology}

This subspecies is named after the country of Malawi, where most of the material was collected.

\section{Material examined}

\section{Holotype}

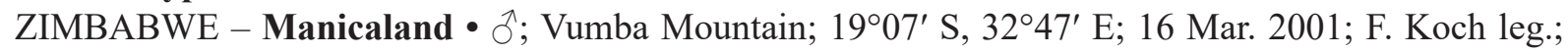
THD-028-ZMB; GenBank CO1 gene: MW538575; GenBank LWR gene: MW582287; ZMB.

\section{Paratypes}

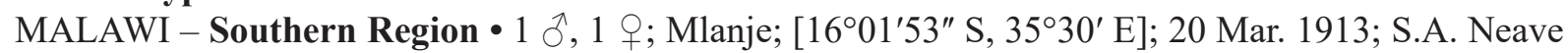
leg.; BMNH $\bullet 1 \hat{\jmath}, 1$ क; same collection data as for preceding but 14 Apr. 1913; BMNH・ $1 \hat{\partial}, 1$ q; same collection data as for preceding but 29 Apr. 1913; BMNH $\bullet 1$; ; same collection data as for preceding

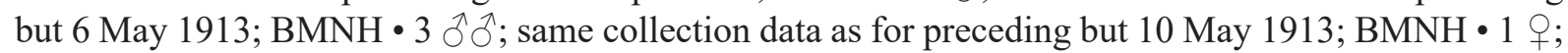
same collection data as for preceding but 19 May 1913; BMNH 11 o , 1 q; same collection data as for preceding but 13 Jun. 1913; BMNH 1 ภ , 4 $O+$; same collection data as for preceding but 1 Jul. 1913;

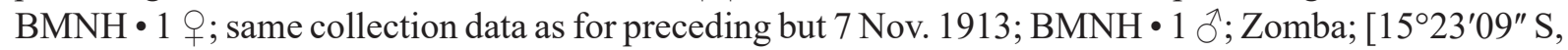

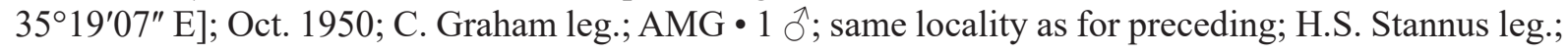
BMNH.

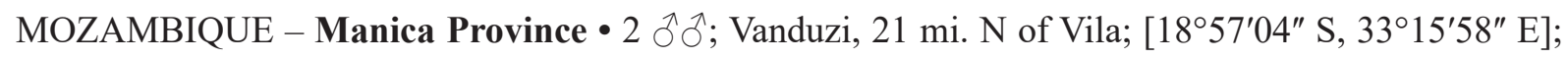
14 Mar. 1958; Pery leg.; CAS.

TANZANIA - Ruvuma Region - 1 ; ;gano (Umgano), Matengo Highlands, WSW of Songea; [1058' S, 345' E]; alt. 1500-1700m; 23 Nov. 1937; F. Zimmer leg.; NHMW • 3 o $\circ$; same locality as for preceding; 20-30 Nov. 1935; Zerny leg.; NHMW • 5 + $\%$; same collection data as for preceding but 1-10 Dec. 1935; NHMW 1 ó; same collection data as for preceding but 1-12 Jun. 1936; NHMW.

ZAMBIA - Central Province • 1 ô; "Alala Plateau, Mkushi distr.”; alt. about 4000 ft; 16 Nov. 1905; S.A. Neave leg.; OUMNH.

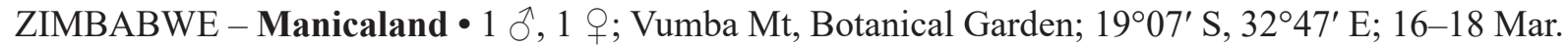
2001; F. Koch leg.; ZMB. 


\section{Description}

Female

Size. $26.7-27.1 \mathrm{~mm}$.

CoLOR. Black, except for basal half of mandible and free clypeal margin, which are ferruginous. Wings uniformly fuscous, with cyan-violet iridescence.

VeSTITURE. Appressed setae on clypeus and paraocular area black, towards lateral clypeal margins and on paraocular area with golden luster. Appressed setae on collar, scutum and propodeal enclosure black. Erect setae on clypeus, paraocular area, collar, scutum and propodeal enclosure black. Erect propodeal setae oriented posteriorly. Lower center of clypeus glabrous. Scutellum densely and coarsely pubescent.

STRUCTURE. Free clypeal margin medially with two broad, indistinct processes, stepped above. Clypeus with indentation in lower center, without carina. Scutellum convex. Metanotum not raised, not bituberculate. $2^{\text {nd }}$ recurrent vein joins markedly proximal from interstitium between submarginal cells II and III. Propodeal enclosure without any notable ridges. Foretarsomere I $2.7 \times$ length of antepenultimate spine. Petiole length $1.7-1.8 \times$ its medial width.

\section{Male}

Size. $26.2-27.1 \mathrm{~mm}$.

CoLor. Black, except for basal half mandible, which is ferruginous. Wings slightly fuscous, with cyanviolet iridescence.

VeSTITURE. Appressed setae on clypeus and paraocular area golden, on collar, scutum and propodeal enclosure black. Erect setae on clypeus black, on paraocular area black intermixed with golden ones, on collar, scutum and propodeal enclosure black, on posterior margin of propodeum silvery-golden. Erect propodeal setae oriented posteriorly. Clypeus with glabrous spot medially at free margin. Scutellum densely and coarsely pubescent. Metasomal sterna II-VII with increasingly dense fringes of black setae.

STRUCTURE. Free clypeal margin simple. Scutellum convex. Metanotum not raised, not bituberculate. $2^{\text {nd }}$ recurrent vein joins markedly proximal from interstitium between submarginal cells II and III. Propodeal enclosure without any notable ridges. Posterior margin of metasomal tergum VII convex. Posterior margin of metasomal sternum VII simple, of metasomal sternum VIII semicircular. Fused penis valvae laterally not widened, with notch, valviceps oriented perpendicularly. Petiole length $1.7-1.9 \times$ its medial width. Flagellomeres IV-VI with broad placoids covering their entire length.

\section{Variation}

Unknown.

\section{Distribution}

Southeastern Africa.

Sphex stadelmanni stadelmanni Kohl, 1895

Figs 16, 21-22, 95, 99 (yellow)

Sphex stadelmanni Kohl, 1895: 67

Chlorion stadelmanni var. integrum Arnold, 1928: 372, o, ô (syntypes: South Africa, KwaZulu-Natal, Scottburgh; Zimbabwe, Manicaland, Chirinda Forest; Mozambique, "Rikatla", R. Stevenson leg.; SAM, TMP, not examined). Syn. nov. 


\section{Differential diagnosis}

Within their species group, members of this species (Fig. 95) closely resemble those of S. abyssinicus and $S$. schoutedeni malawicus subsp. nov., as all three are black with primarily dark vestiture. The males of $S$. stadelmanni s. lat. are most easily distinguishable through the structure of their genitalia. The penis valvae are fused and curved towards the dorsal side, where they form a chalice-like configuration with a slightly widened margin (Figs 21-22). The valvicipites are curved posteroventrally. In S. schoutedeni and its subspecies $S$. s. malawicus subsp. nov., the penis valvae lack a widened margin, and the valvicipites do not curve back toward the ventral side, but continue in dorsal direction (Figs 23-24). In S. abyssinicus, the fused part of the valvae is dorsoventrally enlarged (Fig. 26) and sternum VIII is conspicuously rectangular (Fig. 25).

Males of S. abbotti abbotti (Fig. 88) and S. bohemanni (Fig. 90) are also similar, but can be identified via their incised sternum VII (Figs 19-20). Those of the subspecies S. stadelmanni rufus subsp. nov. (Fig. 97) are characterized by having the central and lower part of the clypeus, as well as trochanter, femur and tibia, ferruginous instead of black.

Females of $S$. stadelmanni stadelmanni are very difficult to distinguish from those of S. abbotti abbotti, as both share uniformly black appressed clypeal and paraocular setae without a differently-colored luster (Figs 15-16). However, the ferruginous color of the mandibles is more extensive and also present on the free clypeal margin in $S$. stadelmanni stadelmanni. In contrast, $S$. abbotti abbotti has the clypeal margin and most of the mandibular base black. In some of the other females of the bohemanni group, the appressed setae on the paraocular area and clypeus are also black, but show a luster of a different color when viewed from certain angles (Figs 14, 17-18).

\section{Material examined}

Holotype

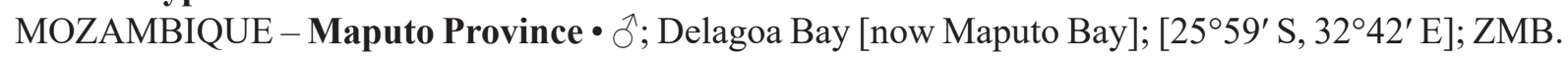

\section{Other material}

ESWATINI - Manzini Region • 1 ô, 1 क; Middleveld, Malkerns Research Station; [26 31'41.7" S, 31¹1'55.5" E]; 16 Mar. 1979; G.G.M. Schulten leg.; RMNH・ 1 §’; same collection data as for preceding but 19 Apr. 1979; RMNH.

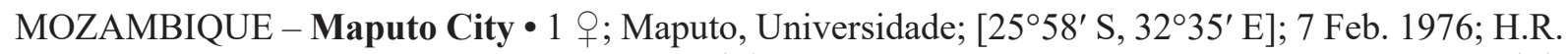

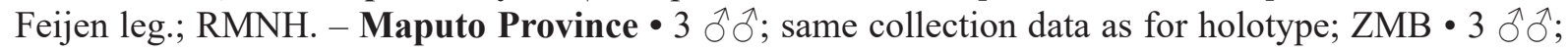
same locality as for holotype; R. Monteiro leg.; ZMB.

SOUTH AFRICA - Gauteng • 1 q; Johannesburg, Bloksberg; [2612'16" S, 2802'44" E]; 1907; C.H.

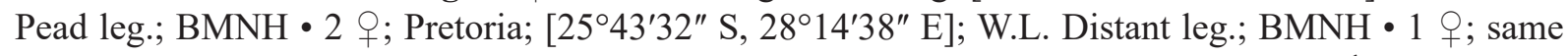
locality as for preceding; 1 Jan. 1924; W. Lingnau leg.; DEI. - KwaZulu-Natal • 1 §; $20 \mathrm{~km} \mathrm{~S}$ of

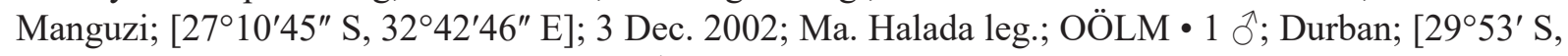

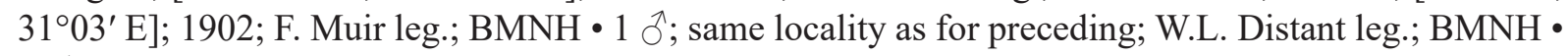

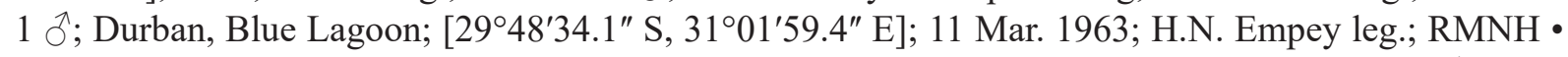

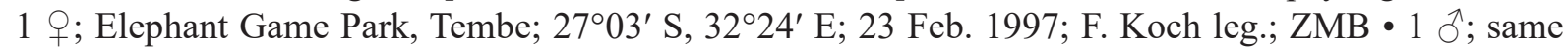
collection data as for preceding but 19 Nov. 1999; ZMB 1 क; Ithala Game Res.; $27^{\circ} 30^{\prime} \mathrm{S}, 31^{\circ} 20^{\prime} \mathrm{E}$;

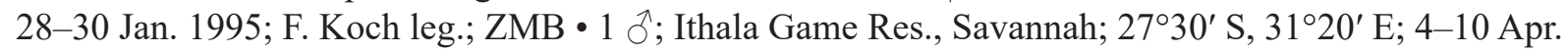

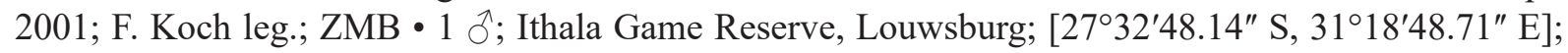
10-23 Dec. 1993; F. Koch leg.; THD-008-ZMB; GenBank LWR gene: MW582288; ZMB • 1 ふै; same collection data as for preceding; ZMB • 1 đ’; Kosi Bay Nature Reserve; $26^{\circ} 58^{\prime} \mathrm{S}, 32^{\circ} 50^{\prime} \mathrm{E}$; 19 Apr. 1999; F. Koch leg.; THD-009-ZMB; GenBank CO1 gene: MW538556; ZMB • 1 đ’; Malvern; [2953'06" S, 
3055'30" E]; 1904; J.P. Cregoe leg.; BMNH • 1 ô, 1 क; Mbazwana; [2730' S, 32³4'44.4" E]; 6 Dec.

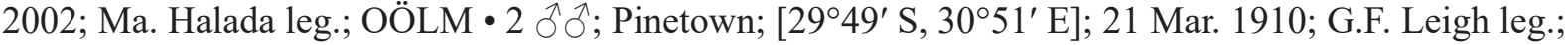

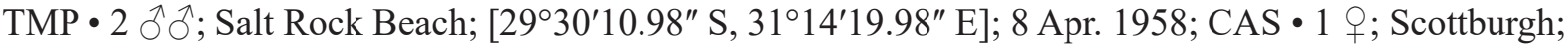

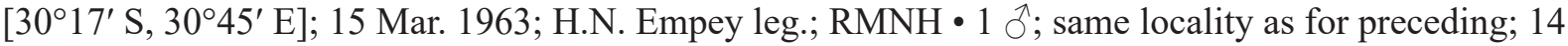
Mar. 1926; R.H.R. Stevenson leg.; BMNH • 1 क; Shongweni Dam; [2951'15" S, 3042'50" E]; 15 Jan. 1976; F.J. Herbst leg.; AMG • 1 đ; ; same locality as for preceding; 25 Feb. 1971; F.L. Farquharson leg.; AMG • 2 우; Umtentweni; 3043' S, 30²8' E; 20 Apr. 1973; H.N. Empey leg.; AMG. - Limpopo •

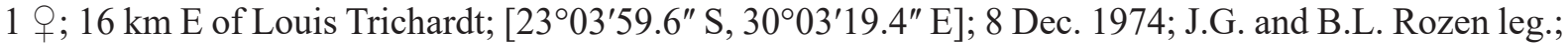
AMNH • 1 क ; Lekgalameetse Nature Reserve; $24^{\circ} 12^{\prime}$ S, 30²0' E; 25-31 Mar. 2001; F. Koch leg.; ZMB • 1 त; same collection data as for preceding; ZMB $\bullet 1$ \%; same collection data as for preceding but 26 Mar.1 Apr. 2001; ZMB • 13 $\widehat{\jmath}, 3$ 우; same collection data as for preceding but 30 Oct.-3 Nov 2010; ZMB.

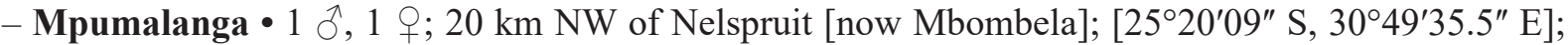

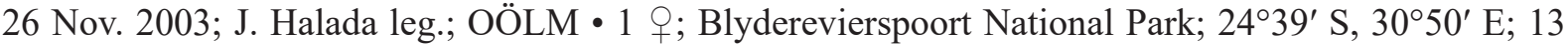
Dec. 1995; F. Koch leg.; ZMB • 2 우; same collection data as for preceding but 10 Nov. 1999; ZMB • 1 q; same collection data as for preceding but 13 Nov. 1999; ZMB • 1 \%; same collection data as for preceding but 1-3 Apr. 2001; ZMB.

ZAMBIA - Northern Province • 1 क; "L. Chambezi V.; Kasama distr.”; alt. 3900 ft; 4-6 May 1908; S.A. Neave leg.; OUMNH.

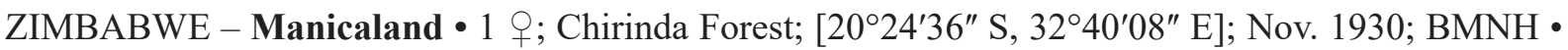
1 क; same collection data as for preceding but Dec. 1935; BMNH $\bullet 1$; ; same locality as for preceding; [20²4'37" S, 3241'57" E]; 20 Oct. 1949; USNM.

\section{Description}

Female

Size. 25.5-28.2 mm.

CoLOR. Black except for basal half of mandible, free clypeal margin and occasionally femora, which are dark ferruginous. Wings uniformly fuscous, with cyan-purple iridescence.

VESTITURE. Appressed and erect setae on clypeus, paraocular area, collar, scutum and propodeal enclosure black. Erect propodeal setae oriented posteriorly. Lower center of clypeus glabrous. Scutellum densely and coarsely pubescent.

STRUCTURE. Free clypeal margin medially with two broad, indistinct processes, stepped above. Clypeus with indentation in lower center, longitudinal carina in upper center barely noticeable. Scutellum convex. Metanotum not raised, not notably bituberculate. $2^{\text {nd }}$ recurrent vein joins markedly proximal from interstitium between submarginal cells II and III. Propodeal enclosure without any notable ridges. Foretarsomere I 2.4-2.6 $\times$ length of antepenultimate spine. Petiole length $1.3-1.7 \times$ its medial width.

Male

Size. 22.9-27.0 $\mathrm{mm}$.

CoLor. Black except for basal half of mandible, which is ferruginous. Wings slightly fuscous, with cyan-purple iridescence.

VeSTITURE. Appressed setae on clypeus and paraocular area brassy, on collar, scutum and propodeal enclosure black. Erect setae on clypeus, paraocular area, collar, scutum and propodeal enclosure black, on posterior margin of propodeum brassy in some specimens. Erect propodeal setae oriented posteriorly. 
Free clypeal margin glabrous. Scutellum densely and coarsely pubescent. Metasomal sterna II-VII with increasingly dense fringes of black setae.

STRUCTURE. Free clypeal margin simple. Scutellum convex. Metanotum not raised, not bituberculate. $2^{\text {nd }}$ recurrent vein joins markedly proximal from interstitium between submarginal cells II and III. Propodeal enclosure without any notable ridges. Posterior margin of metasomal tergum VII convex. Posterior margin of metasomal sternum VII simple, of metasomal sternum VIII semicircular. Fused penis valvae laterally widened, without notch, valviceps oriented posterodorsally. Petiole length $1.6-2.2 \times$ its medial width. Flagellomeres IV-VI with broad placoids covering their entire length.

\section{Variation}

Unknown.

\section{Distribution}

Southeastern Africa.

\section{Remarks}

Of Sphex stadelmanni stadelmanni, only the holotype is known. It differs from members of the subspecies S. s. integer (Arnold, 1928), which was described about 30 years later, in a single character: S. s. stadelmanni possesses a very notable broad incision on the posterior margin of tergum VII, while $S$. s. integer has tergum VII entire. We have studied several other males from the same locality as the type, presumably collected in a similar time period, but all of them, as well as every other specimen we examined that belonged to $S$. stadelmanni s. lat. were either $S$. s. integer or $S$. s. rufus subsp. nov. The morphological disparity in the holotype of S. stadelmanni stadelmanni might have been the result of malformation during ontogeny. This hypothesis is corroborated by the fact that all of the other terga of the specimen appear to be deformed in a similar way, albeit asymmetrically and only on the right side. It seems as though in this case, the emarginations are placed more centrally on a tergum the further posterior it is, so the symmetrical incision on the final tergum could fit with this theory. We propose that $S$. s. integer becomes a synonym of $S$. s. stadelmanni, as the holotype of $S$. s. stadelmanni is presumably merely an aberration of the same species, though the name has priority. Perhaps future DNA analyses will reveal more information, but with the methods used in this project we cannot hope to generate meaningful data from this over 120 -year-old specimen.

Sphex stadelmanni rufus subsp. nov. urn:1sid:zoobank.org:act:4873E4CA-248F-4293-852F-0144E8E2E1D9

Figs 96-97, 99 (purple)

\section{Differential diagnosis}

Females of S. stadelmanni rufus subsp. nov. (Fig. 96) are easy to recognize within the bohemanni group because of their predominantly ferruginous clypeus, femora and tibiae. These areas are largely black in other species, with at most the free clypeal margin being ferruginous.

In males (Fig. 97), the ferruginous color on the clypeus is less extensive and sometimes barely visible, but the leg coloration is concurrent with that of the female and often sufficient for identification as well. Another character that differs from $S$. stadelmanni stadelmanni is the color of the appressed facial setae, which is silvery-white in the nominate species, but brassy in this subspecies. Both are identical in genital structure, and the shape of their penis valvae (Figs 21-22) reliably separates them from other species in the group. 


\section{Etymology}

'Rufus' is a Latin adjective meaning 'red', which refers to the conspicuously-colored legs and clypeus of this taxon.

\section{Material examined}

\section{Holotype}

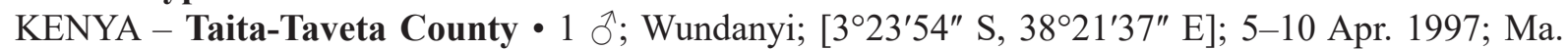
Halada leg.; THD-014-OOLM; GenBank CO1 gene: MW538562; OÖLM.

\section{Paratypes}

KENYA - Taita-Taveta County $\bullet 5 \hat{\partial} \hat{\partial}, 1$; same collection data as for holotype; OÖLM $\bullet 3 \hat{\delta} \hat{\delta}$, 2 + $ᄋ$; same collection data as for holotype but 18-22 Mar. 1997; OÖLM.

TANZANIA • 1 q; "German East Africa”; F. Fischer leg.; ZMB. - Kilimanjaro Region • 1 ภ, 2 q $q$; "Kilimanjaro"; [ $3^{\circ} 04^{\prime} 33^{\prime \prime}$ S, 37²1'12" E]; Jan. 1906; Schröder leg.; ZMB • 1 q; Gonja; [4¹6'09.3" S, 3802'54.9" E]; 10 Jan. 1906; Schröder leg.; ZMB. - Morogoro Region • 1 q; "Usaramo, Rufidji, Khutusteppe"; [747'59.4" S, 37²9'13.1" E]; Oct.-Nov. 1896; Götze leg.; ZMB. - Tanga Region • 1 O; early 1904; A. Karasek leg.; ZMB • 1 Ō, 1 +; Dec. 1905; Schröder leg.; ZMB • 1 \%; "East Usambara”;

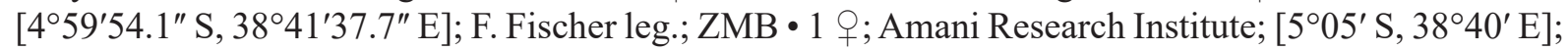
2 Mar. 1908; J. Vosseler leg.; ZMB • 1 o; Bumbuli; [452' S, 38²8’ E]; 15 Dec. 1902; Meinhof leg.; ZMB.

\section{Description}

Female

Size. $25.0-31.5 \mathrm{~mm}$.

CoLOR. Black except for the following, which are ferruginous: basal half of mandible, clypeus excepting dorsal and lateral margin, basal half of scape, trochanters, femora and tibiae. Wings uniformly fuscous, with purple-bluish iridescence.

VeSTITURE. Appressed setae on clypeus and paraocular area black with ferruginous-golden luster, on collar, scutum and propodeal enclosure black. Erect setae on clypeus, paraocular area, collar, scutum and propodeal enclosure black. Erect propodeal setae oriented posteriorly. Lower center of clypeus glabrous. Scutellum densely and coarsely pubescent.

STRUCTURE. Free clypeal margin medially with two broad, indistinct processes, stepped above. Clypeus with inconspicuous indentation in lower center, longitudinal carina in upper center barely noticeable. Scutellum convex. Metanotum slightly raised, not notably bituberculate. $2^{\text {nd }}$ recurrent vein joins markedly proximal from interstitium between submarginal cells II and III. Propodeal enclosure without any notable ridges. Foretarsomere I 2.5-2.8 $\times$ length of antepenultimate spine. Petiole length $1.3-1.9 \times$ its medial width.

\section{Male}

SizE. $24.5-28.2 \mathrm{~mm}$.

CoLor. Black except for the following, which are ferruginous: basal half of mandible, longitudinal stripe medially on clypeus, basal half of scape, trochanters, femora and tibiae. Wings slightly fuscous, with violet iridescence. 
VestiTURE. Appressed setae on clypeus and paraocular area brassy, on collar, scutum and propodeal enclosure black, on posterior margin of propodeum silvery-golden. Erect setae on clypeus black, on paraocular area black intermixed with silvery ones, on collar, scutum and propodeal enclosure black, on posterior margin of propodeum silvery-golden. Erect propodeal setae oriented posteriorly. Free clypeal margin medially glabrous. Scutellum densely and coarsely pubescent. Sterna II-VII laterally with increasingly dense fringes of black setae, sternum VIII uniformly with shorter, erect, ferruginous-brown setae which are longer laterally and posteriorly.

STRUCTURE. Free clypeal margin simple. Scutellum convex. Metanotum slightly raised, not bituberculate. $2^{\text {nd }}$ recurrent vein joins markedly proximal from interstitium between submarginal cells II and III. Posterior margin of metasomal tergum VII convex. Propodeal enclosure without any notable ridges. Posterior margin of metasomal sternum VII simple, of metasomal sternum VIII semicircular. Petiole length 1.9-2.1 $\times$ its medial width. Flagellomeres IV-VI with broad placoids covering their entire length, flagellomere VII sometimes with moderately broad placoid covering approximately its basal half.

\section{Variation}

Unknown.

\section{Distribution}

Eastern Africa.

\section{Species of the gaullei group}

The gaullei group contains the following five species: Sphex decipiens, S. gaullei, S. jansei, S. pruinosus and $S$. schmideggeri sp. nov.

Members of this group can be recognized through a combination of anteriorly-oriented, curved erect propodeal setae and a convex scutellum. The orientation of the setae is best visible near the lateral margins of the propodeal enclosure and separates this group from the umtalicus- and meridionalis groups, where the erect propodeal setae are oriented perpendicularly but slightly curved towards anterior as well. Almost all of the species of the gaullei group can still be distinguished through their silvery or golden propodeal setae, whereas most members of the other two groups have them black. Sphex decipiens is a notable exception and can thus easily be mistaken as belonging to the meridionalis group, since, in accordance with the members of that group, it lacks the very dense and coarse setae on the scutellum that are present in the umtalicus group.

The presence of a convex scutellum can be used to delimit members of the gaullei group from those of the satanas group, which share the presence of anteriorly-oriented erect propodeal setae. The difference between the two is very easily recognizable in females (Fig. 5-6), but less pronounced in males. Most of them can be distinguished through the color of the clypeus and the color and density of its vestiture, as all males of the satanas group have the clypeus black (sometimes with the exception of having the free margin ferruginous) and the appressed and erect setae silvery-white without any conspicuous glabrous spots. In contrast, $S$. jansei, S. gaullei and $S$. schmideggeri sp. nov. have approximately the lower clypeal half brightly ferruginous, and the first two of them also sport golden erect facial setae. Sphex decipiens has the clypeus uniformly black, but its erect facial setae are also black in contrast to the silvery-white setae of the males in the satanas group. In addition, all males from the gaullei group have a notable glabrous area on the central and lower part of the clypeus, with one exception. The clypeus of male $S$. pruinosus resembles that of the satanas group members, so the easiest character for distinguishing this species is its elongated petiole, the length of which is around $3.5 \times$ its medial width (see Introduction for measurement details). In comparison, this coefficient is between 1.6 and 2.8 for all other males from both groups. 
Sphex decipiens Kohl, 1895

Figs 28, 100-101, 112 (red)

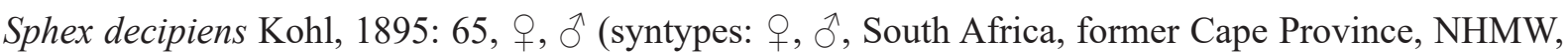
examined).

\section{Differential diagnosis}

This species is very distinct from the other members of the gaullei group, as both sexes have the clypeus black except near the free clypeal margin (Figs 28, 101), the scutellum black, the appressed facial setae silvery and the erect propodeal setae black. Therefore, $S$. decipiens is more likely to be mistaken for a species of the umtalicus group or, especially in the case of male specimens, one of the meridionalis group. Still, sequence data show that it is closely related to $S$. jansei.

One of the most diagnostically useful characters present in both sexes of members of the umtalicus group is a densely pubescent scutellum. Species of the meridionalis- and gaullei groups only have very sparse vestiture on the scutellum. As mentioned in the introductory remarks, this can even be seen when most of the setae have been lost due to abrasion, as the scutellar impression will usually still show the original state. Additionally, members of S. decipiens (Fig. 100) are distinguished from those of the meridionalis group by having the erect propodeal setae oriented anteriorly, as is the case for all species in the gaullei- and satanas groups. In members of the meridionalis- and umtalicus groups, the setae are more perpendicular and merely curved towards anterior (Figs 3-4). As this can be difficult to see, though, it may be easier to rely on other characters like the singular narrow placoid present only on flagellomere $\mathrm{V}$ of the antenna in males of the meridionalis group, whereas males of $S$. decipiens have a placoid on flagellomere IV and on occasion also on parts of flagellomeres III and V.

\section{Material examined}

\section{Syntypes}

SOUTH AFRICA・1 ô, 1 +; “Capland”; NHMW.

\section{Other material}

AFRICA 11 क; Cap, Cafrerie; $1865 ;$ MNHN $\bullet 1$ ते; same collection data as for preceding; MNHN.

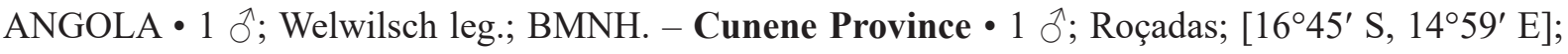
30 Mar. 1972; BMNH.

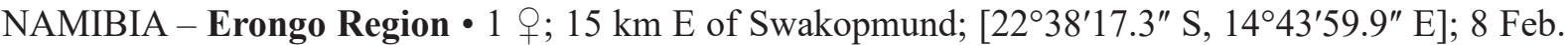
1993; J. Gusenleitner leg.; OÖLM • 1 ô; $^{\wedge} 20 \mathrm{~km} \mathrm{NW}$ of Uis; $21^{\circ} 04^{\prime} \mathrm{S}, 1^{\circ} 41^{\prime} \mathrm{E}$; 24 Mar. 2014; J. Halada

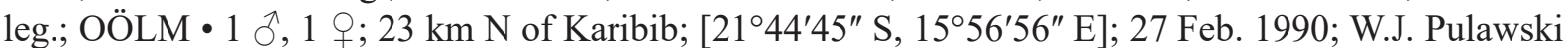

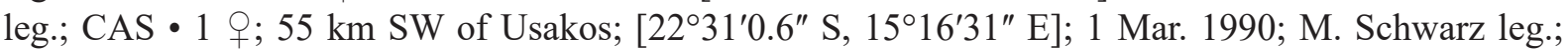

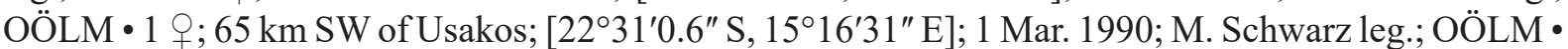

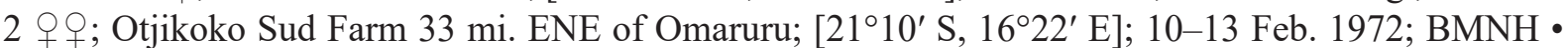
1 ; Phillips Ranch, 5 mi. N of Usakos; [21 ${ }^{\circ} 55^{\prime} 35^{\prime \prime}$ S, 15³4'37" E]; 24 Oct. 1967; E.S. Ross and A.R. Stephen leg.; CAS. - Hardap Region • 1 क; 15 km S of Rehoboth; $23^{\circ} 28^{\prime}$ S, $17^{\circ} 07^{\prime}$ E; 20 Mar. 2014;

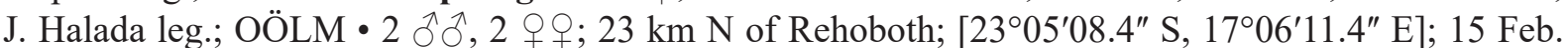

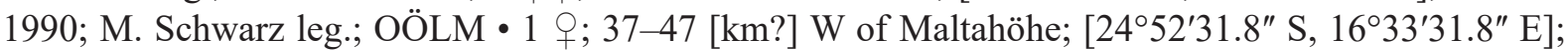

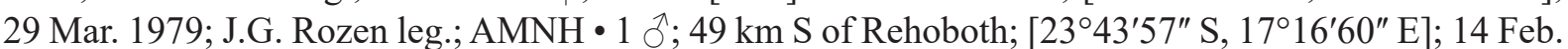

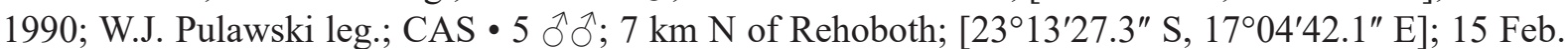

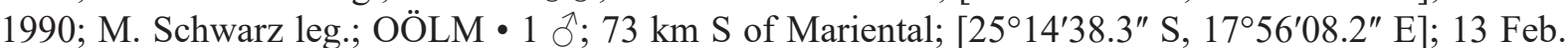

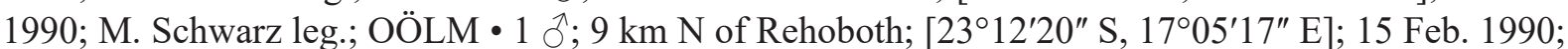

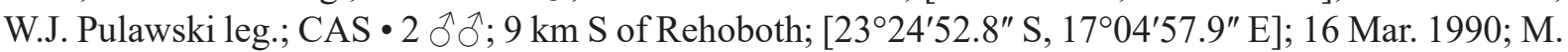




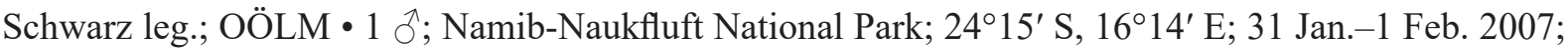

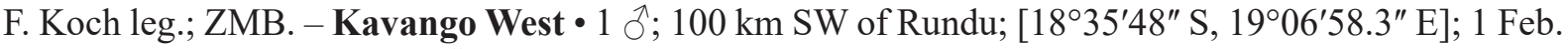
1993; J. Gusenleitner leg.; OÖLM • 1 q; 80 km SW of Rundu; [18 $28^{\prime} 17.9^{\prime \prime}$ S, 19¹4'1.9" E]; 25 Jan. 1993; M. Schwarz leg.; OÖLM. - Khomas Region • 1 đo; $18 \mathrm{~km} \mathrm{~W}$ of 1237/C26 junction; $23^{\circ} 09^{\prime}$ S, 16²' E; 11 Mar. 1999; F.W. and S.K. Gess leg.; AMG • 1 o ; Regenstein 15 mi. SSW of Windhoek;

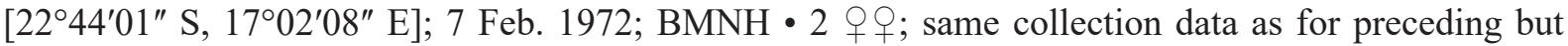
8 Feb. 1972; BMNH • 1 क; S of Okasewa; 22 34'53" S, 18¹1'15" E; $12-17$ Feb. 2010; J. Oehlke leg.; coll. Jacobs. - Kunene Region • 1 क; 15 km SE of Outjo; [20¹1'47.9" S, 16²16'01" E]; 3 Mar. 1990; M. Schwarz leg.; OÖLM • 1 क; Otjitambi Farm 27 mi. ESE of Kamanjab; [1949’ S, 15¹0' E]; 14 Feb. 1972; BMNH • 2 우; same collection data as for preceding but 13-15 Feb. 1972; BMNH. - Oshikoto

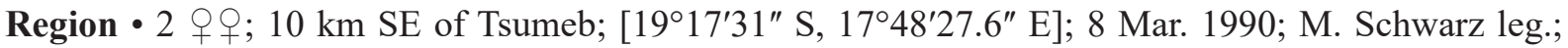

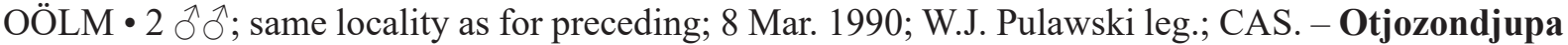

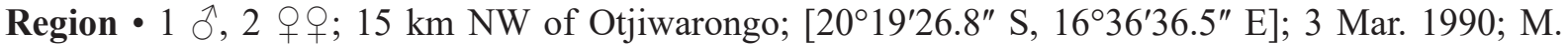

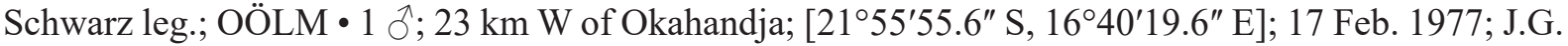

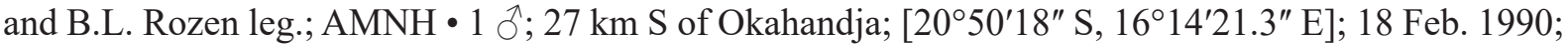

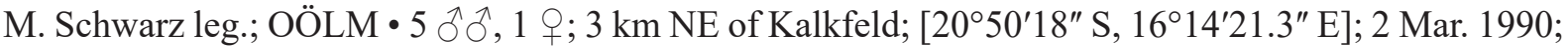

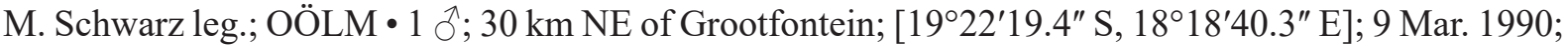

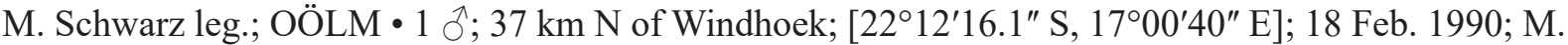

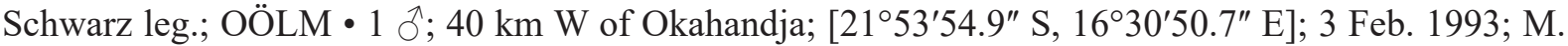
Schwarz leg.; OÖLM • 1 क; 90 km NE of Grootfontein; [1859'50.5" S, 1844'22.3" E]; 16 Jan. 1993;

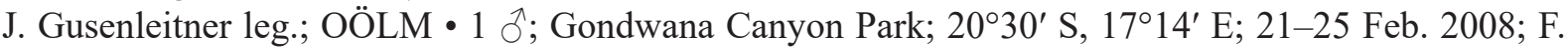

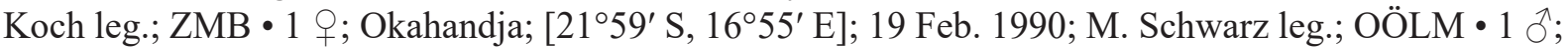

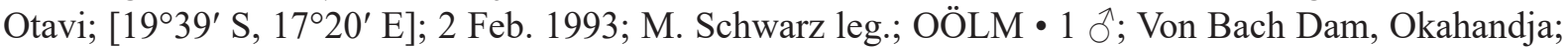

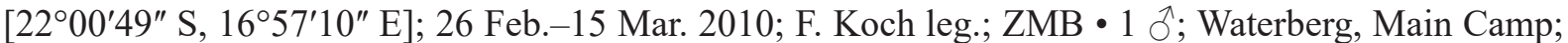

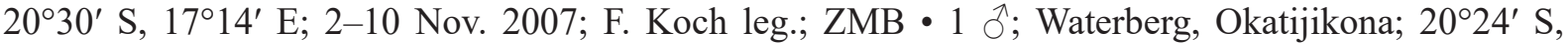
17²4' E; 27-28 Feb. 2010; F. Koch leg.; ZMB.

SOUTH AFRICA • 1 đ; Cape Province, Huguenot; Feb. 1932; BMNH. - Eastern Cape • 1 đ;

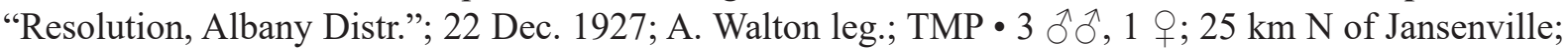

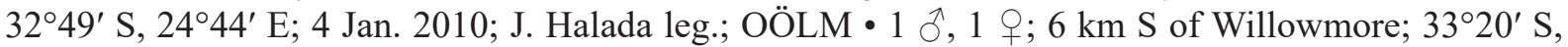

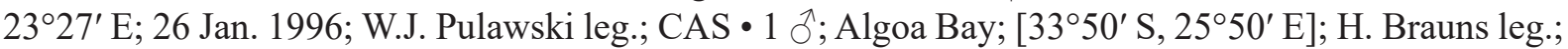

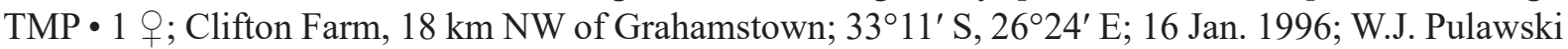

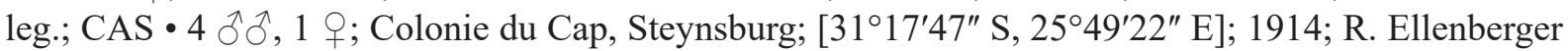

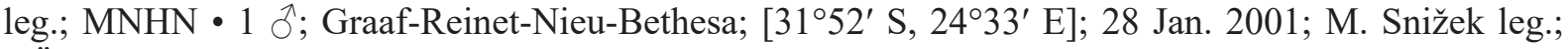

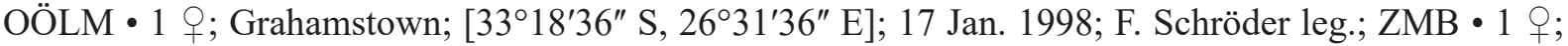

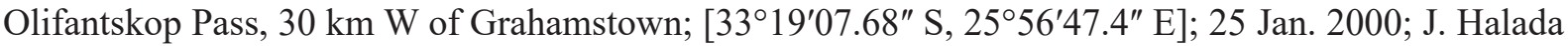

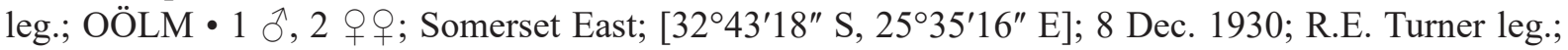

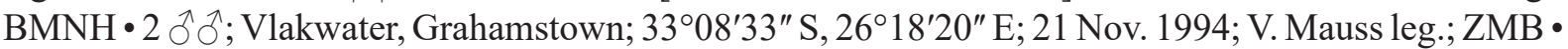

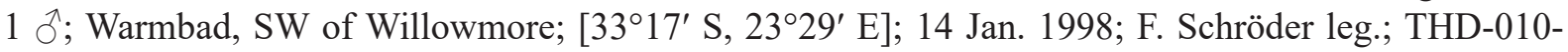

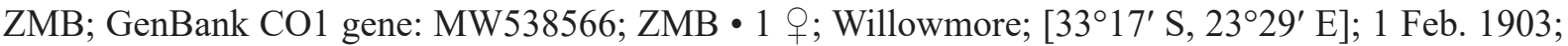
H. Brauns leg.; BMNH • 1 क; same collection data as for preceding but 17 Feb. 1903; TMP • 1 q; same collection data as for preceding but $25 \mathrm{Feb} .1903$; BMNH $\bullet 1$ đ̊; same collection data as for preceding but 6 Dec. 1906; TMP • 1 o ; same collection data as for preceding but 20 Dec. 1911; TMP • 1 § ; same collection data as for preceding but $10 \mathrm{Jan} .1912 ; \mathrm{BMNH} \bullet 1$ \%; same locality as for preceding; 1904; G.

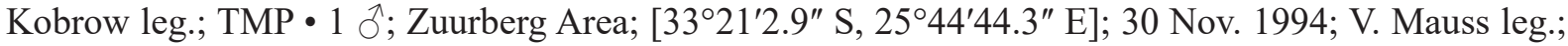

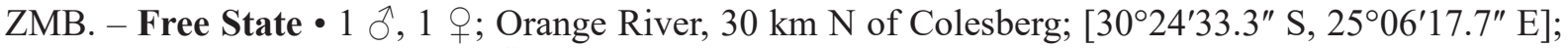

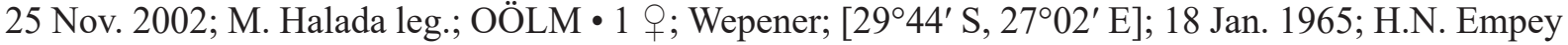
leg.; RMNH. - North West • 1 ○े, 2 우; 20 km W of Bothaville, Klerksdorp, Vaal River; [26 $57^{\prime} 0.9^{\prime \prime}$ S,

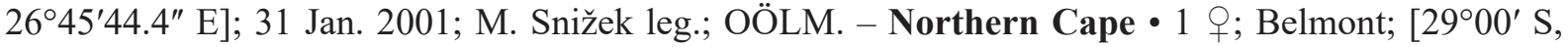

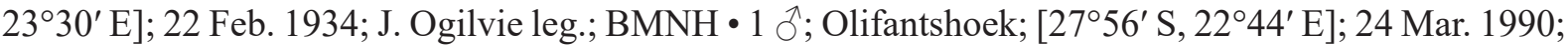




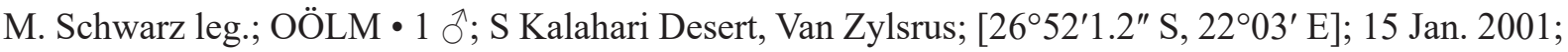
M. Snižek leg.; OÖLM. - Western Cape • $1 \delta^{\lambda}$; 18 mi. SE of Touwsrivier; [33⒉ $7^{\prime} 52.7^{\prime \prime}$ S, 20¹8'52.4" E]; 12 Nov. 1966; J.G. Rozen leg.; AMNH • 1 ơ; Barrydale; [3354'29" S, 2043' E]; 16 Dec. 2002; M.

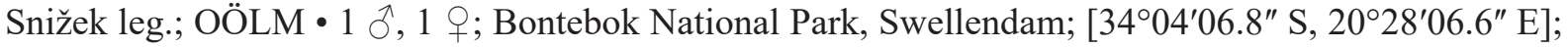
2-3 Jan. 1993; F. Koch leg.; ZMB • 1 क ; same collection data as for preceding; THD-001-ZMB; GenBank EF-1 $\alpha$ gene: MW558235; GenBank LWR gene: MW582286; ZMB • 1 §; Cape Town, Kloof

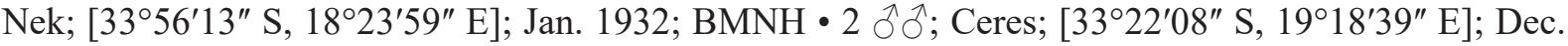

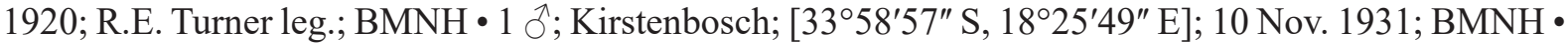

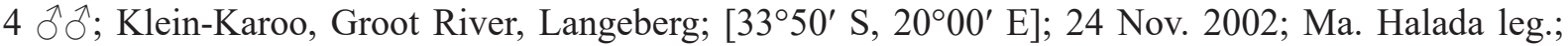

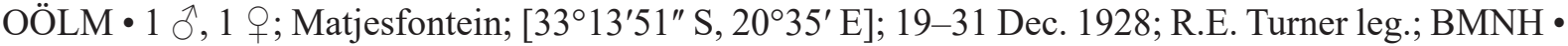

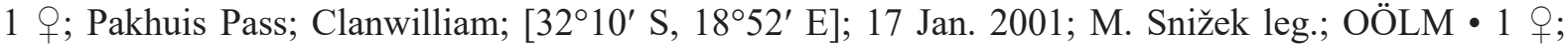

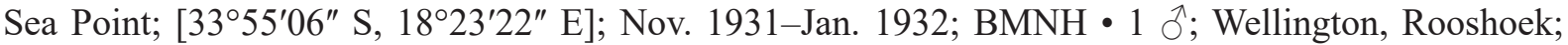

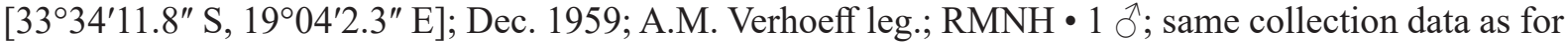
preceding but Jan. 1960; RMNH • 1 क; Worcester; [3338'47" S, 19²6'54" E]; Dec. 1933; R.E. Turner leg.; BMNH.

\section{Description}

\section{Female}

Size. 20.4-23.9 mm.

CoLOR. Black except for the following, which are ferruginous: basal half of mandible, free clypeal margin, occasionally antenna from scape up to flagellomere $\mathrm{V}$ and occasionally legs from distal half of femur onward. Wings uniformly fuscous, with violet iridescence.

VeSTITURE. Appressed setae on clypeus and paraocular area silvery, on collar, scutum and propodeal enclosure black. Erect setae on clypeus, paraocular area, collar, scutum and propodeal enclosure black. Erect propodeal setae oriented anteriorly. Clypeus with glabrous spot in lower center. Scutellum sparsely and finely pubescent.

StRUCTURE. Free clypeal margin simple, stepped medially. Clypeus without indentation or carina. Scutellum convex. Metanotum slightly raised, not notably bituberculate. $2^{\text {nd }}$ recurrent vein joins markedly proximal from interstitium between submarginal cells II and III. Propodeal enclosure without any notable ridges. Foretarsomere I 1.7-2.0 $\times$ length of antepenultimate spine. Petiole length 1.9-2.0 $\times$ its medial width.

\section{Male}

SizE. $16.6-20.5 \mathrm{~mm}$.

COLOR. Black, except for ferruginous stripe in center of mandible. Wings fuscous, with violet iridescence.

VestiTURE. Appressed setae on clypeus and paraocular area silvery, on collar, scutum and propodeal enclosure black. Erect setae on clypeus, paraocular area, collar, scutum and propodeal enclosure black. Erect propodeal setae oriented anteriorly. Lower part of clypeus glabrous. Scutellum densely and finely pubescent.

StRUCTURE. Free clypeal margin simple. Scutellum convex. Metanotum slightly raised, not notably bituberculate. $2^{\text {nd }}$ recurrent vein joins markedly proximal from interstitium between submarginal cells II and III. Propodeal enclosure without any notable ridges. Posterior margin of metasomal tergum VII convex. Posterior margin of metasomal sternum VII simple, of metasomal sternum VIII very slightly concavely emarginate. Penis valvae without conspicuous modifications. Petiole length $1.9-2.2 \times$ its medial width. Flagellomere III with narrow placoid covering it to varying extent, flagellomere IV with 


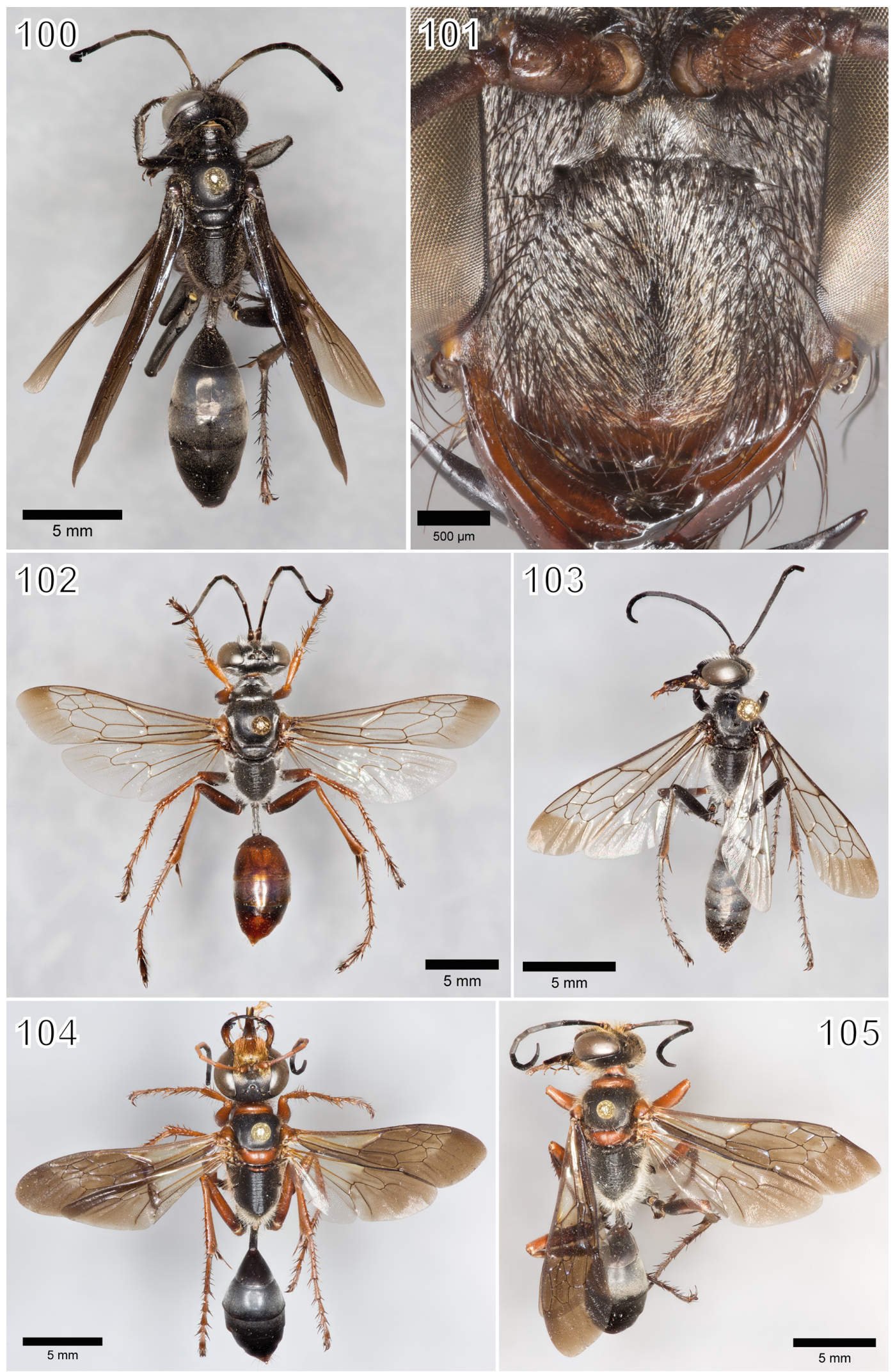

Figs 100-105. 100-101. Sphex decipiens Kohl, 1895. 102-103. S. pruinosus Germar, 1817. 104105. S. gaullei Berland, 1927. 100, 103, 105. Habitus of ${ }^{\top}$. 101. Frontal view of $q$. 102, 104. Habitus of $q$. 
narrow placoid nearly covering entire length, flagellomere $\mathrm{V}$ with placoid absent or narrow, covering it to varying extent.

\section{Variation}

In females, antennae and legs can be partially ferruginous, and males can have the lower clypeal area more or less ferruginous.

\section{Distribution}

Southern and southwestern Africa.

Sphex gaullei Berland, 1927

Figs 6, 31-32, 104-105, 112 (yellow)

Sphex gaullei Berland, 1927: 151, ㅇ, ô.

Sphex rufiscutis laevigatus Arnold, 1951: 145, ㅇ. Junior primary homonym of Sphex laevigatus Rossi, 1794. Syn. nov.

\section{Differential diagnosis}

This species is very similar to $S$. jansei and has frequently been mistaken for it in the past, as until now there were no identification keys that featured both species. The morphological differences between the two are subtle, but undeniable.

Females of S. jansei (Fig. 106) and S. gaullei (Fig. 104) are characterized through their ferruginous clypeus with golden setae, their convex, sparsely pubescent, ferruginous scutellum, and their almost completely black scutum, petiole and metasoma. Sphex schmideggeri sp. nov. (Fig. 108) is similar, but has the scutum, petiole and metasomal tergum I predominantly ferruginous. The main characters that distinguish $S$. gaullei from $S$. jansei are the color of the erect propodeal setae, which is brownish-gray in S. gaullei and silvery-white in $S$. jansei, and the extent of the reddish coloration on the body. Sphex jansei has the pronotal lobe mostly black, with the posterior margin ferruginous at most, and some specimens also have a black collar. In S. gaullei, the collar and pronotal lobe are predominantly ferruginous.

Regarding males, S. jansei (Fig. 107) and S. gaullei (Fig. 105) both have approximately the lower half of the clypeus ferruginous and the remainder black, with the erect propodeal setae being silvery. What separates them is the fact that $S$. gaullei has the distal half of the fore- and hindwing notably infuscate (Fig. 32). In contrast, the wings of S. jansei are hyaline in the cellular area and only the apical margin is fuscous (Fig. 30). Both species have the erect facial setae golden, whereas male S. schmideggeri sp. nov. have them silvery.

\section{Material examined}

Lectotype (designated here)

CENTRAL AFRICAN REPUBLIC - Nana-Grébizi • $\bigcirc$; Fort-Crampel [now Kaga-Bandoro]; MNHN.

\section{Paralectotypes}

CENTRALAFRICAN REPUBLIC - Nana-Grébizi • 1 ô; same collection data as for holotype; MNHN • 1 ; ; same collection data as for holotype; BMNH.

\section{Other material}

BURKINA FASO - Cascades Region • 1 q; Tingréla, Banfora; [10³8’31.7" N, 449'50.9" W]; 20 Dec.

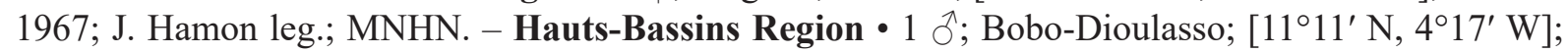




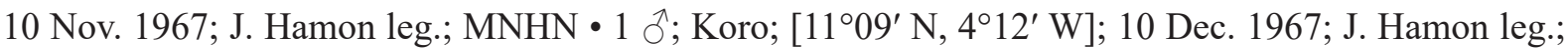
MNHN • 1 đ̃; same collection data as for preceding but 13 Dec. 1967; MNHN.

DEMOCRATIC REPUBLIC OF THE CONGO - Nord-Ubangi - 2 q $q$; Yakoma; [4 $05^{\circ} 53^{\prime \prime} \mathrm{N}$, $22^{\circ} 26^{\prime} 45^{\prime \prime}$ E]; 12 Feb. 1932; H.J. Brédo leg.; MRAC • 6 우; same collection data as for preceding but 17 Feb. 1932; MRAC.

ETHIOPIA 1 1ठ, 1 q; 1882; Raffray leg.; MNHN.

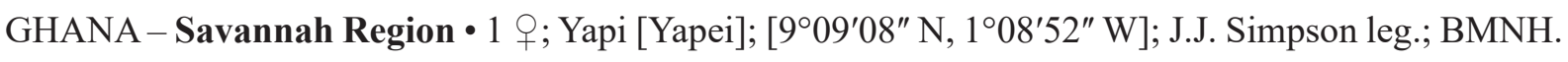

MALI - Bamako Region • 1 क; Barainba, NE of Bamako; [12³9’ N, 800’ W]; Aug. 1991; G. Popov leg.; BMNH. - Mopti Region • 1 + , holotype of Sphex rufiscutis laevigatus Arnold, 1951; near Sofara; [1401' N, 4²14' W]; 3 Sep. 1944; K.M. Guichard leg.; BMNH.

NIGERIA • 1 क; Michika-Bama; 6 Dec. 1979; G. Popov leg.; BMNH. - Oyo State • 1 q; Fashola near Oyo; [754' N, 347' E]; 15 Nov. 1975; J.T. Medler leg.; USNM.

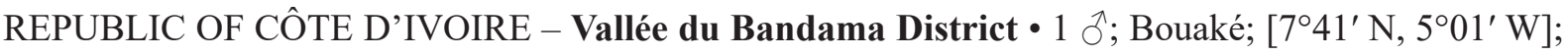
1977; P.M. Elsen leg.; MRAC.

RWANDA - Western Province • 1 q; Ishwa, Lake Albert; [2²3'04" S, 28 55'58" E]; Sep. 1935; H.J. Brédo leg.; MRAC.

SENEGAL・ 1 đ̊; "Between Tambacounda and Kaffrine"; 27-30 Dec. 1967; M. Coruet leg.; MNHN.

SUDAN - South Kordofan • 1 q; Talodi, Nouzi, Nuba Mountains; [10³7'56" N, 30²2'46" E]; Nov. 1921; F. Moysey leg.; BMNH.

\section{Description}

Female

Size. $19.7-23.1 \mathrm{~mm}$.

COLOR. Black except for the following, which are ferruginous: basal half of mandible, labrum, clypeus, scape, pedicel, flagellomeres I-V, collar, tegula, pronotal lobe, prepectus, scutellum, metanotum, forecoxa and legs from trochanter onward, except apical half of claw. Basal wing half often with yellow tinge, apical half fuscous with violet iridescence.

VeSTITURE. Appressed setae on clypeus, paraocular area and scutum golden, on propodeal enclosure silvery-golden. Erect setae on clypeus and paraocular area ferruginous-golden, on collar and scutum ferruginous, on propodeal enclosure brownish-gray. Erect propodeal setae oriented anteriorly. Lower center of clypeus glabrous. Scutellum densely and finely pubescent.

STRUCTURE. Free clypeal margin slightly notched medially, stepped above. Clypeus without indentation or carina. Scutellum convex. Metanotum raised, slightly bituberculate. $2^{\text {nd }}$ recurrent vein joins markedly proximal from interstitium between submarginal cells II and III. Propodeal enclosure with eight to twelve sharply distinct transversal ridges. Foretarsomere I 1.7-1.8 $\times$ length of antepenultimate spine. Petiole length $2.0-2.4 \times$ its medial width.

\section{Male}

Size. $17.0-21.2 \mathrm{~mm}$.

COLOR. Black except for the following, which are ferruginous: basal half of mandible, labrum, scape, pedicel ventrally, proximal third of flagellomere I ventrally, lower half of clypeus, legs from femur or 
tibia onward up to tarsomere II, collar, tegula and dorsal part of scutellum. Basal wing half hyaline, apical half fuscous with violet iridescence.

VeSTITURE. Appressed setae on clypeus, paraocular area, collar, scutum and propodeal enclosure silvery. Erect setae on clypeus and paraocular area golden, on collar ferruginous-golden, on scutum and propodeal enclosure silvery. Erect propodeal setae oriented anteriorly. Lower part of clypeus glabrous. Scutellum densely and finely pubescent.

StRUCTURE. Free clypeal margin simple. Scutellum convex. Metanotum raised, slightly bituberculate. $2^{\text {nd }}$ recurrent vein joins markedly proximal from interstitium between submarginal cells II and III. Propodeal enclosure with indistinct ridges. Posterior margin of metasomal tergum VII convex. Posterior margin of metasomal sternum VII simple, of metasomal sternum VIII concavely emarginate. Penis valvae without conspicuous modifications. Petiole length $2.2-2.4 \times$ its medial width. Flagellomeres III + IV with narrow placoids covering their entire length.

\section{Variation}

Unknown.

\section{Distribution}

Western to central Africa.

Sphex jansei Cameron, 1910

Figs 2, 29-30, 106-107, 112 (purple)

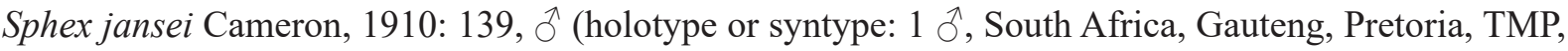
not examined).

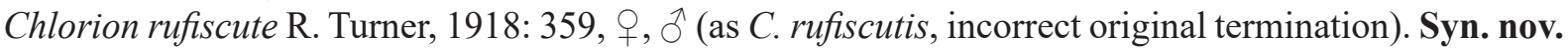
Sphex mochii Giordani Soika, 1942: 197, o (as S. Mochii, incorrrect original capitalization) (holotype:

, Ethiopia, Oromia Region, km 46 on Harrar-Dire Daua Road, depository?, not examined). Syn. nov.

\section{Differential diagnosis}

Females of $S$. jansei (Fig. 106) are distinguished through their hyaline cellular wing area (Fig. 29) and silvery-white propodeal setae, whereas those of $S$. gaullei (Fig. 104) and S. schmideggeri sp. nov. (Fig. 108) have the propodeal setae brownish-grey. The cellular wing area of $S$. gaullei is somewhat infuscate (Fig. 31), while that of $S$. schmideggeri sp. nov. has a distinct yellow tinge.

Males of S. jansei (Fig. 107), as well as those of S. gaullei (Fig. 105), are distinguished from those of the remaining group members by their combination of a notable ferruginous clypeus and golden erect facial setae. Sphex jansei has the cellular forewing area hyaline and only a fuscous spot on the anterior part of the apical hindwing margin (Fig. 30), whereas S. gaullei has most of the cellular forewing area and all of the hindwing apex infuscate (Fig. 32).

\section{Material examined}

AFRICA $• 3$ + $9 ; \mathrm{ZMB}$.

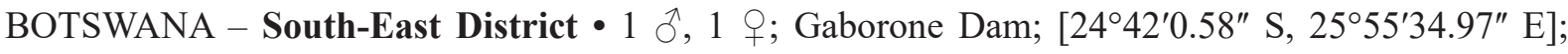
28 Dec. 1977; H.R. Feijen leg.; RMNH.

CENTRAL AFRICAN REPUBLIC - Ouham-Pendé • 1 क; Bozoum; [6 $6^{\circ} 19^{\prime} 02^{\prime \prime}$ N, 16 $16^{\circ} 22^{\prime} 42^{\prime \prime}$ E]; 30 Apr. 1914; S. Tessmann leg.; ZMB. 
KENYA - Homa Bay County • 1 đ; Gembe Hills, near seasonal stream; $0.48933^{\circ} \mathrm{S}, 34.24333^{\circ} \mathrm{E}$; $15-$ 22 Jan. 2005; R. Copeland leg.; ICIPE. - Nakuru County • 1 गे; Mount Longonot Crater; [0 $54^{\prime} 55^{\prime \prime}$ S, 36²7'25" E]; Dec. 1911; Ch. Alluaud and Jeannel leg.; MNHN.

MALAWI • 1 ภ, 1 \%; NW shore of Lake Nyasa, between Florence Bay and Karonga; 30 Jun.-6 Jul. 1910; S.A. Neave leg.; BMNH. - Northern Region • 1 '; Karonga; [956' S, 3356' E]; 7-11 Jul. 1910;

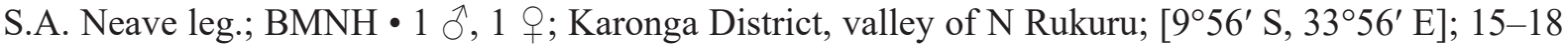

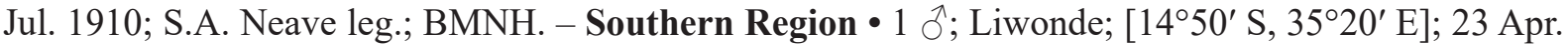
1975; G.G.M. Schulten leg.; RMNH.

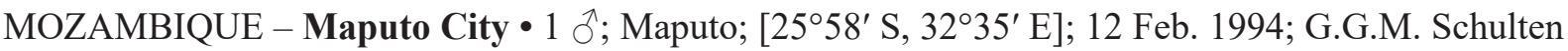

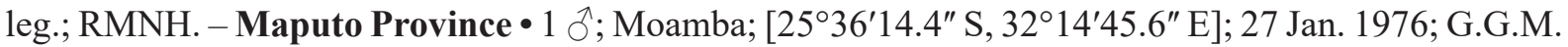

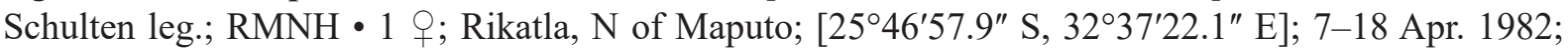
G.G.M. Schulten leg.; RMNH.

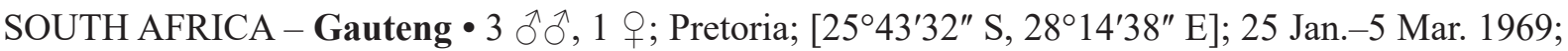

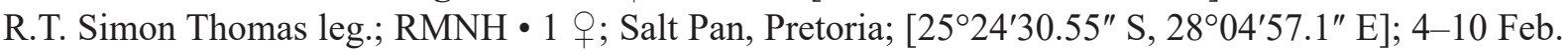
1929; G. van Son leg.; TMP • 1 क; same collection data as for preceding but 14 Mar. 1956; TMP. -

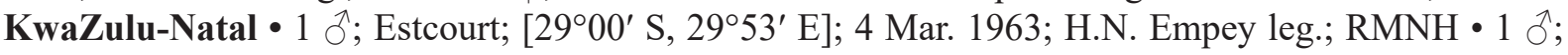
Ithala Game Res.; 2730' S, 3120' E; 28-30 Jan. 1995; F. Koch leg.; THD-025-ZMB; Genbank CO1 gene: MW538561; ZMB • 1 q; Lake St Lucia, False Bay; [2800'31.3" S, 32²1'39.9" E]; 13-17 Feb.

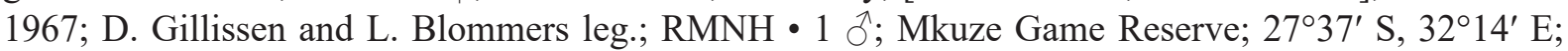
26 Feb.-3 Mar. 1987; A.J. Weaving leg.; AMG • 1 ð , 2 우; same collection data as for preceding but 8-12 Mar. 1987; AMG • 1 O; Weenen; [2850'57" S, 3004'38" E]; Feb. 1925; H.P. Thomasset leg.; BMNH •

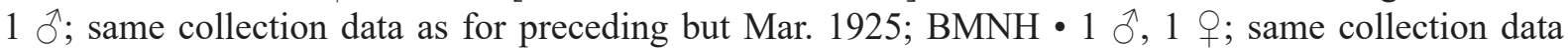
as for preceding but Jan.-Mar. 1927; BMNH. - Limpopo - 1 ô; Punda Milia, Kruger National Park;

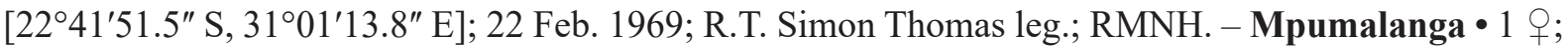

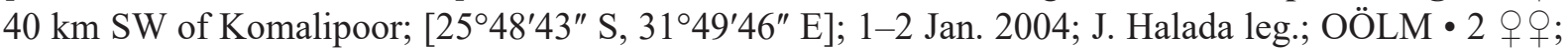

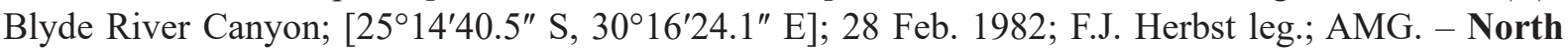

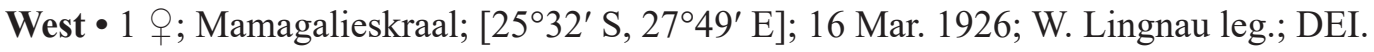

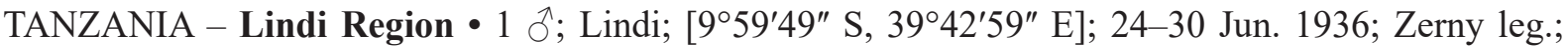

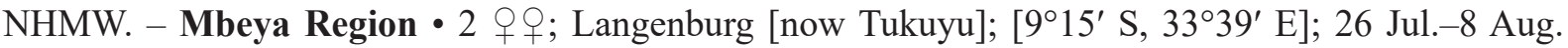
1898; F. Fülleborn leg.; ZMB • 1 क; same collection data as for preceding but 3 Sep.-4 Nov. 1898; ZMB. - Morogoro Region • 1 q; $50 \mathrm{~km} \mathrm{SW}$ of Morogoro; 6 ${ }^{\circ} 50^{\prime} \mathrm{S}, 37^{\circ} 15^{\prime} \mathrm{E}$; 12 Jan. 2007; J. Halada leg.; OÖLM. - Rukwa Region • 1 क; Kafokola, Rukwa Valley, SW Tanganyika; [ $8^{\circ} 00^{\prime} \mathrm{S}, 32^{\circ} 00^{\prime} \mathrm{E}$ ]; 9 Jun. 1952; O.W. Richards leg.; BMNH.

ZAMBIA • 1 q; "Luangwa to Petauke"; 14-17 Sep. 1910; S.A. Neave leg.; BMNH 1 1 , holotype of Chlorion rufiscute R. Turner, 1918; "Sinapunge"; 13 Feb. 1911; Silverlock leg.; BMNH・ 1 ○े, 1 q; same collection data as for preceding; BMNH. - Eastern Province $\bullet 1$; ; near mouth of Lusangazi River;

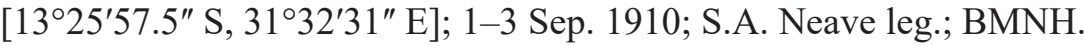

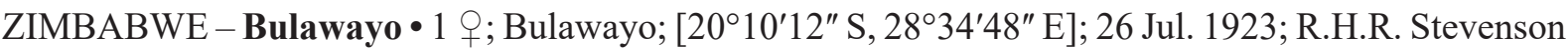

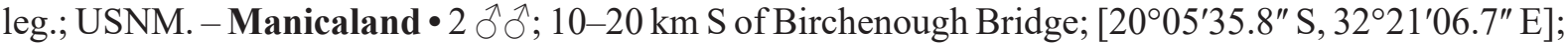
24 Dec. 1998; M. Snižek leg.; OÖLM. - Mashonaland West • 1 q; 15 km NW of Makuti; [16 ${ }^{\circ} 12^{\prime}$ S, $29^{\circ} 09^{\prime}$ E]; 12 Apr. 1985; J. Gusenleitner leg.; NHMW • 1 đ; Sanyati Camp, Lake Kariba; $\left[17^{\circ} \mathrm{S}, 28^{\circ} \mathrm{E}\right]$; 8-10 Jan. 1985; A.J. Weaving leg.; AMG. - Matabeleland North • 1 ภे; Lonely Mine; [19³0'06" S,

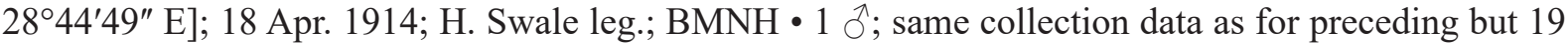


Sep. 1914; BMNH • 1 क; Matetsi; [1805' S, 2607' E]; 1 Apr. 1934; R.H.R. Stevenson leg.; RMNH • 1 क; same collection data as for preceding but 28 Oct. 1934; BMNH 1 o'; Victoria Falls; [17 $56^{\prime}$ S, 2550' E]; 14 Jan. 1969; F.J. Herbst leg.; AMG. - Matabeleland South • 1 o; Beit Bridge; [22 $13^{\circ}$ S,

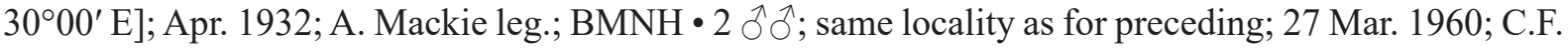
Jacot-Guillarmod leg.; AMG.

\section{Description}

\section{Female}

Size. 21.2-25.0 mm.

CoLOR. Black except for the following, which are ferruginous: basal half of mandible, scape, pedicel, flagellomeres I-IV, clypeus, legs from trochanter onward, excluding apical half of claw, and occasionally collar dorsally, tegula, scutellum, dorsal part of metanotum and apical segment of metasoma. Cellular area of fore- and hindwing hyaline, apical margin fuscous.

VestituRE. Appressed and erect setae on clypeus and paraocular area golden, on collar, scutum and propodeal enclosure silvery. Erect propodeal setae oriented anteriorly. Clypeus medially with vertical glabrous stripe. Scutellum densely and finely pubescent.

StRUCTURE. Free clypeal margin stepped medially, with slight indentation above. Scutellum convex. Metanotum slightly raised, not markedly bituberculate. $2^{\text {nd }}$ recurrent vein joins markedly proximal from interstitium between submarginal cells II and III. Propodeal enclosure without any notable ridges. Foretarsomere I 1.8-2.0 $\times$ length of antepenultimate spine. Petiole length $2.4-2.7 \times$ its medial width.

\section{Male}

SizE. 20.1-22.8 mm.

CoLOR. Black except for the following, which are ferruginous: basal half of mandible, scape, pedicel ventrally, lower part of clypeus, legs from trochanter onward, excluding posterodorsal portion of trochanter and femur as well as apical half of claw, tegula and dorsal part of scutellum. Cellular area of forewing hyaline, apical margin fuscous. Hindwing hyaline.

VeSTITURE. Appressed setae on clypeus and paraocular area silvery-golden, on collar, scutum and propodeal enclosure silvery. Erect setae on clypeus and paraocular area golden, on collar, scutum and propodeal enclosure silvery. Erect propodeal setae oriented anteriorly. Clypeus medially with vertical glabrous stripe. Scutellum densely and finely pubescent.

STRUCTURE. Free clypeal margin simple. Scutellum convex. Metanotum slightly raised, not bituberculate. $2^{\text {nd }}$ recurrent vein joins markedly proximal from interstitium between submarginal cells II and III. Propodeal enclosure with indistinct ridges. Posterior margin of metasomal tergum VII convex. Posterior margin of metasomal sternum VII simple, of metasomal sternum VIII concavely emarginate. Penis valvae without conspicuous modifications. Petiole length $2.3-2.8 \times$ its medial width. Flagellomeres III + IV with narrow placoids covering their entire length.

\section{Variation}

Unknown.

\section{Distribution}

Southeastern to eastern Africa. 

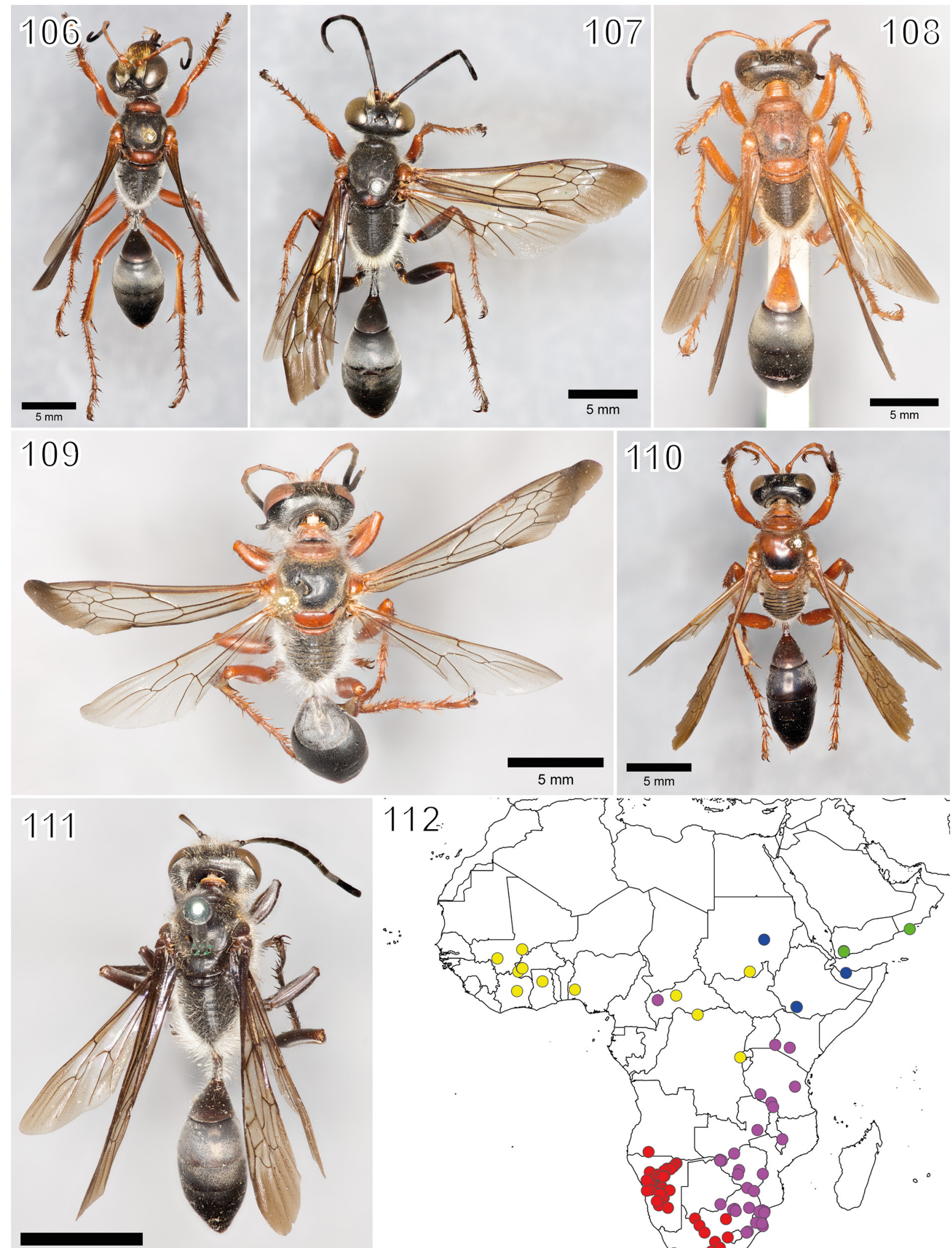

$5 \mathrm{~mm}$
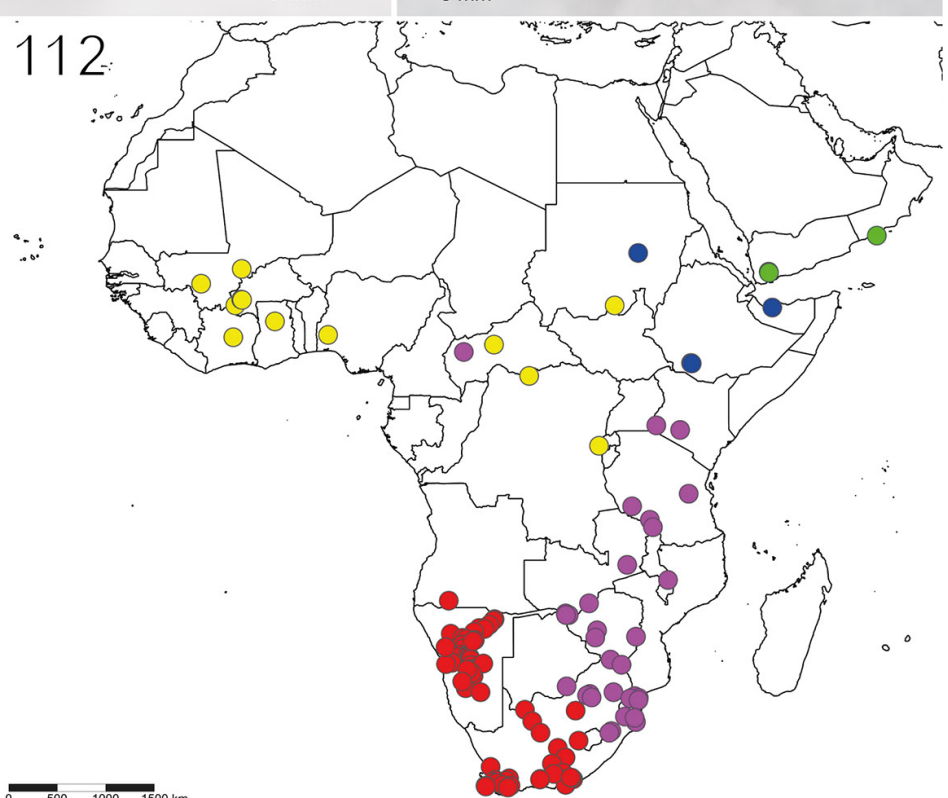

Figs 106-112. 106-107. Sphex jansei Cameron, 1910. 108. S. schmideggeri sp. nov. 109. S. pseudosatanas sp. nov. 110-111. S. rufoclypeatus sp. nov. 106, 108-110. Habitus of ${ }^{+}$. 107, 111. Habitus of ${ }^{\lambda}$. 112. Geographic distribution of S. decipiens Kohl, 1895 (red), S. pruinosus Germar, 1817 (blue), S. gaullei Berland, 1927 (yellow), S. jansei (purple) and S. schmideggeri sp. nov. (green). 


\section{Remarks}

Besides the original description, there are almost no other publications that mention diagnostic characters for this species. Brauns (1917) placed it in the genus Isodontia Patton, 1880, which, as correctly noted by Arnold (1928), must be incorrect due to the length of the petiole being described as only one-fourth longer than the hind coxa. Arnold also indicated that $S$. jansei may be synonymous to $S$. rufiscutis, but he was not able to certify this because of "the description of the colour [...] [being] too confused". He stated that the type specimen of $S$. jansei could no longer be found in the TMP. Despite receiving material from the collection and having specifically requested the type specimen to be sent, it was not included in the loan, and further inquiries remained unanswered. Thus, we were forced to rely solely on the original description.

Part of the reason why it is difficult to interpret the color characters given by Cameron (1910) is that there likely were some important words omitted in the text; an attempt at correcting this issue is presented here:

"Black; ... mandibles, except the teeth, [red, as well as] the apex of clypeus, the centre broadly (the red colour extending near to the middle in the centre), the sides narrowly, antennal scape, tegulae, and the legs, except the [following which are all black:] coxae, greater part of the trochanters, a streak on the basal outer half of the fore femora, the basal three-fourths of the middle behind, and the hinder with more than three-fourths, ..."

Assuming these changes concur with Cameron's intended description, the characters mentioned very closely match those we observed in males of $S$. rufiscutis and even rule out conspecificity with the similar $S$. gaullei, which is found in the equatorial regions of Africa, on account of the wing coloration. The geographic distribution of $S$. jansei and $S$. rufiscutis fit together as well, and all other species from southern Africa, an area from which we examined a large amount of material, are quite distinct. Therefore, we propose that jansei becomes the valid name for this species.

The type material of Sphex mochii Giordani Soika, 1942, is apparently lost, so the original description by Giordani Soika (1942) is the only basis we can use to discern its taxonomic identity.

The original description stated that the female of S. mochii is similar to that of S. rufiscutis (R. Turner, 1918) (= S. jansei Cameron, 1910). The scutellum was said to be more convex than in S. jansei, and the scutellum and metanotum are medially impressed. The petiole was described as being much shorter than in S. pruinosus. The wings of S. mochii were stated to be hyaline with the apical margin of the forewing infuscate, and the color pattern described corresponds with that of $S$. jansei and three other African species that are superficially similar (S. gaullei, S. pseudosatanas sp. nov. and S. occidentalis sp. nov.). Even so, the description cannot refer to $S$. pseudosatanas sp. nov., as this species has the scutellum markedly flatter than in S. jansei. Additionally, S. occidentalis sp. nov. can be ruled out due to its having uniformly fuscous wings. Finally, $S$. gaullei does not entirely match the description either, because a large part of its cellular wing area is infuscate (Fig. 31). Also, the scutellum of S. jansei and S. gaullei is more or less identical. We conclude that S. mochii is identical to $S$. jansei (= rufiscutis), which is in contrast to Giordani Soika's original interpretation.

Sphex pruinosus Germar, 1817

Figs 102-103, 112 (blue)

Sphex pruinosus Germar, 1817: 261, đ̊ (as S. pruinosa, incorrect original termination).

Sphex scioensis Gribodo, 1879: 343, ㅇ (as S. Scioensis, incorrect original capitalization) (holotype not examined). Synonymized with Sphex pruinosus by Kohl 1890: 391. 


\section{Differential diagnosis}

Among the African Sphex, this species is easily recognized. Both sexes have a very long petiole (Figs 102-103), its length usually being more than three times its medial width. The only other species with a comparably long petiole is $S$. cinerascens (Fig. 38), both sexes of which have a conspicuous process medially on the free clypeal margin, similar to the one present in males of the umtalicus group. Sphex cinerascens has a yellow tinge on the basal half of the forewing, and its metasoma is completely black, whereas $S$. pruinosus lacks the yellow tinge and, in females, often has the anterior half of the metasoma somewhat ferruginous.

\section{Material examined}

\section{Holotype or syntype}

CROATIA - Split-Dalmatia County • ${ }^{\wedge}$; Spalatro [now Split]; ZMB.

\section{Other material}

AFRICA・ 1 đ̊; "Africa”; DEI.

ETHIOPIA - Southern Nations, Nationalities, and Peoples' Region $\bullet 1$; $10 \mathrm{~km} \mathrm{SE}$ of Konso;

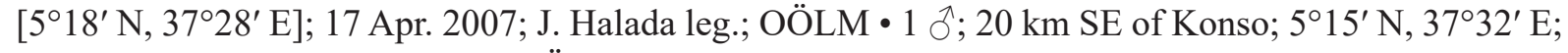
11-13 May 2015; J. Halada leg.; OÖLM.

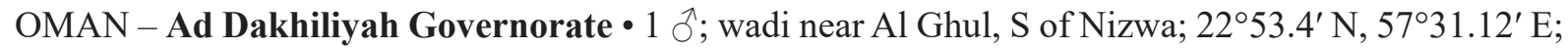
10 Dec. 2003; M. Ohl leg.; THD-036-ZMB; GenBank EF-1 $\alpha$ gene: MW558236; GenBank LWR gene: MW582275; ZMB.

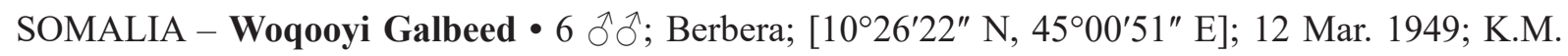
Guichard leg.; BMNH.

SUDAN - Khartoum State • 1 \%; Khartoum; [15³0'02" N, 32³3'36" E]; 4 Apr. 1964; L. Razoux

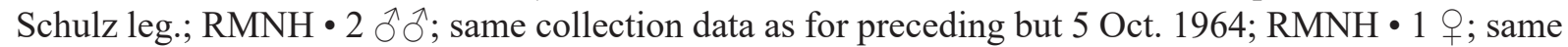
locality as for preceding; 18 Nov. 1986; G.G.M. Schulten leg.; RMNH・1 $O$; same collection data as for preceding but 4 Dec. 1991; RMNH.

\section{Description}

\section{Female}

Size. $21.3-23.2 \mathrm{~mm}$.

CoLOR. Black except for the following, which are ferruginous: basal half of mandible, scape, tegula, legs from femora onward and metasoma. Wings hyaline, apical margin of forewing fuscous.

VeSTITURE. Appressed and erect setae on clypeus, paraocular area, collar, scutum and propodeal enclosure silvery. Erect propodeal setae oriented anteriorly. Clypeus without glabrous spot. Scutellum densely and finely pubescent.

Structure. Free clypeal margin with small notch medially, not stepped above. Clypeus without indentation and carina. Scutellum convex. Metanotum slightly raised, not notably bituberculate. $2^{\text {nd }}$ recurrent vein joins markedly proximal from interstitium between submarginal cells II and III. Propodeal enclosure with 5-8 transversal ridges. Foretarsomere I 1.4-2.0 $\times$ length of antepenultimate spine. Petiole length $3.2-3.3 \times$ its medial width.

\section{Male}

SizE. $15.6-18.2 \mathrm{~mm}$. 
COLOR. Black except for ferruginous stripe in center of mandible. Wings hyaline, apical margin of forewing fuscous.

VeSTITURE. Appressed and erect setae on clypeus, paraocular area, collar, scutum and propodeal enclosure silvery. Erect propodeal setae oriented anteriorly. Clypeus usually without glabrous spot. Scutellum densely and finely pubescent.

StRUCTURE. Free clypeal margin simple. Scutellum convex. Metanotum slightly raised, not notably bituberculate. $2^{\text {nd }}$ recurrent vein joins markedly proximal from interstitium between submarginal cells II and III. Propodeal enclosure with 3-5 indistinct transversal ridges. Posterior margin of metasomal tergum VII convex. Posterior margin of metasomal sternum VII simple, of metasomal sternum VIII concavely emarginate. Petiole length $3.0-3.8 \times$ its medial width. Flagellomeres III-VI with broad placoids covering their entire length.

\section{Variation}

Unknown.

\section{Distribution}

Northeastern Africa, Mediterranean region, Arabian Peninsula to India.

Sphex schmideggeri sp. nov. urn:Isid:zoobank.org:act:EA676305-B0D0-492E-8270-485BEADA1E3B

Figs 108, 112 (green)

\section{Differential diagnosis}

This species (Fig. 108) somewhat resembles S. paulinierii (Fig. 39) in its color pattern, having the wings more or less yellowish with dark apices, and having the petiole, scutellum, metanotum and metasomal segment I ferruginous. However, both species are structurally very different, as $S$. paulinierii is much larger, has the erect setae on the propodeal enclosure very short and has the structure otherwise known as a spiracular groove modified in a way that it is disassociated from the spiracle; we therefore opted to place it in its own subgenus. Sphex schmideggeri sp. nov., on the other hand, has the typical traits of a gaullei group member, namely anteriorly-oriented erect propodeal setae and a convex scutellum.

From the other members of the group, it is distinguished through its ferruginous petiole and metasomal base. Specifically, females differ from those of S. decipiens and S. pruinosus (Fig. 102) in having the clypeus nearly completely ferruginous and the appressed and erect facial setae golden. Those of $S$. jansei (Fig. 106) and S. gaullei (Fig. 104) are distinguishable through having the cellular forewing area hyaline without any tinge (Fig. 29) and apically infuscate (Fig. 31), whereas S. schmideggeri sp. nov. has the cellular forewing area hyaline with a yellow tinge. Males are unique among those of the gaullei group in their combination of having a largely ferruginous clypeus and silvery-white appressed and erect facial setae.

\section{Etymology}

We dedicate this species to Dr Christian Schmid-Egger, who was very kind to provide us with specimens from his collection and their sequence data.

\section{Material examined}

\section{Holotype}

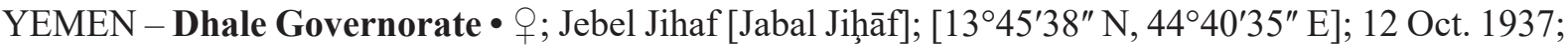
H. Scott and E.B. Britton leg.; BMNH. 
Paratypes

OMAN - Dhofar Governorate 1 o'; Jabal Samhan, $13 \mathrm{~km} \mathrm{~N}$ of Mirbat; $17^{\circ} 06^{\prime} 40^{\prime \prime}$ N, 54 $42^{\prime} 40^{\prime \prime}$ E; 4 Sep. 2019; C. Schmid-Egger leg.; BC-ZSM-HYM-30269-D08; coll. Schmid-Egger • 1 क; same collection data as for preceding; coll. Schmid-Egger.

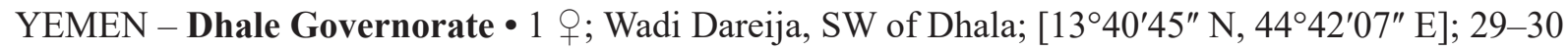
Sep. 1937; H. Scott and E.B. Britton leg.; BMNH.

\section{Description}

\section{Female}

Size. $18.4-22.9 \mathrm{~mm}$.

COLOR. Ferruginous except for the following, which are black: apical half of mandible, head except clypeus, scape, pedicel, flagellomeres VI-IX, posterior half of scutum, episternal sulcus, propodeal enclosure, apical half of claw and metanotum except segment I. Cellular wing area yellowish, apex infuscate.

VeSTITURE. Appressed and erect setae on clypeus, paraocular area, collar, scutum and propodeal enclosure rich or pale golden. Erect propodeal setae oriented anteriorly. Lower center of clypeus glabrous. Scutellum densely and finely pubescent.

STRUCTURE. Free clypeal margin simple, stepped medially. Clypeus without indentation or carina. Scutellum convex. Metanotum raised slightly, not notably bituberculate. $2^{\text {nd }}$ recurrent vein joins markedly proximal from interstitium between submarginal cells II and III. Propodeal enclosure with indistinct ridges. Foretarsomere I 1.8-2.0 $\times$ length of antepenultimate spine. Petiole length $2.3-2.4 \times$ its medial width.

\section{Male}

SIZE. $18.7 \mathrm{~mm}$.

CoLOR. Black except for the following, which are ferruginous: clypeus except dorsolateral margin, basal half of mandible, scape, pedicel, base of flagellomere I, anterior part of pronotum, lower half of propoleuron, collar dorsally, prepectus, posterior half of pronotal lobe, tegula, scutellum, metanotum, petiole, metasomal segment I and legs excluding apical half of claw. Cellular wing area hyaline, with inconspicuous yellow tinge, apex infuscate.

VeSTITURE. Appressed and erect setae on clypeus, paraocular area, collar, scutum and propodeal enclosure silvery. Erect propodeal setae oriented anteriorly. Central and lower part of clypeus glabrous. Scutellum densely and finely pubescent.

STRUCTURE. Free clypeal margin simple. Scutellum convex. Metanotum slightly raised, not bituberculate. $2^{\text {nd }}$ recurrent vein joins markedly proximal from interstitium between submarginal cells II and III. Propodeal enclosure with indistinct ridges. Posterior margin of metasomal tergum VII convex. Posterior margin of metasomal sternum VII simple, of metasomal sternum VIII concavely emarginate. Penis valvae without conspicuous modifications. Petiole length $2.6 \times$ its medial width. Flagellomeres III + IV with narrow placoids covering their entire length.

\section{Variation}

In females, the anterior half of the scutum and the ventral part of the mesopleuron can be black instead of ferruginous.

\section{Distribution}

Southern and southeastern Arabian Peninsula. 


\section{Species of the satanas group}

The satanas group contains the following four species and one subspecies: Sphex pseudosatanas sp. nov., S. rufoclypeatus sp. nov., S. satanas, S. satanas memnon subsp. nov. and S. socotrensis sp. nov.

Females of this group are easy to recognize, as all of them have a very flat scutellum (Fig. 5). All other female Sphex from the Afrotropical region have a more convex scutellum (Fig. 6), the sole exception being $S$. paulinierii. However, its enormous size, conspicuous coloration and very short propodeal setae make that species unmistakable.

Males in the group are characterized by their uniformly black clypeus (excepting S. socotrensis sp. nov., which has the free margin ferruginous) combined with uniformly silvery setae on their entire body. Members of the gaullei group, which share the anteriorly-oriented erect propodeal setae, have a significant amount of the clypeus ferruginously-colored, or their erect setae are not silvery-white. The one exception with a black clypeus and uniformly silvery setae, $S$. pruinosus, is easily recognized by its long petiole, the length of which is greater than 3 times the medial width (see Introduction). In comparison, that ratio is at most 2.5 in all members of the satanas group.

Sphex pseudosatanas sp. nov.

urn:1sid:zoobank.org:act:71C639FE-C171-457B-86DA-757EE7CFC5BF

Figs 109, 118 (red)

\section{Differential diagnosis}

Females of this species are easy to recognize within the satanas group. In contrast to those of $S$. satanas s. lat. (Figs 113-114) and S. socotrensis sp. nov. (Fig. 116), they have both the scutellum and the metanotum uniformly ferruginous (Figs 109), a character which they share with female S. rufoclypeatus sp. nov. (Fig. 110). However, S. rufoclypeatus sp. nov. has the clypeus and the anterior half of the scutum ferruginous, whereas the upper half of the clypeus and the entire scutum are black in S. pseudosatanas sp. nov.

Identification of males is more difficult. While male $S$. socotrensis sp. nov. stand out due to their ferruginous legs (Fig. 117), S. satanas satanas (Fig. 115), S. pseudosatanas sp. nov. and S. rufoclypeatus sp. nov. (Fig. 111) all have a black integument and silvery setae. The most reliable characters to distinguish them are a combination of wing infuscation and placoid pattern. The male of $S$. satanas satanas has uniformly fuscous forewings and largely fuscous hindwings, whereas in S. pseudosatanas sp. nov., only the apex of the forewing is fuscous. Sphex rufoclypeatus sp. nov. has slightly infuscate forewings and hyaline hindwings. Sphex satanas satanas and $S$. pseudosatanas sp. nov. share the pattern of narrow placoids on the proximal half or third of flagellomeres IV-VI, whereas S. rufoclypeatus sp. nov. has broad placoids covering the entire length of flagellomeres III-VI.

\section{Etymology}

'Pseudo-' is a prefix derived from the Greek adjective 'pseudếs' meaning false; 'pseudosatanas' thus refers to the fact that this species can be confused with the similar $S$. satanas.

\section{Material examined}

\section{Holotype}

KENYA - Laikipia County - O; Laikipia, Mpala Research Centre; [0 $0^{\circ} 17^{\prime} 33^{\prime \prime}$ N, 36 $53^{\prime} 53^{\prime \prime}$ E]; 20 Jan. 2005; P. Lenguya leg.; THD-029-BMNH; Genbank CO1 gene: MW538569; GenBank EF-1 $\alpha$ gene: MW558238; GenBank LWR gene: MW582278; BMNH. 
Paratypes

ETHIOPIA - Southern Nations, Nationalities, and Peoples' Region - 1 ${ }_{+}$; Arba Minch; $6^{\circ} 00^{\prime} \mathrm{N}$, 37³3' E; Nov. 2011; M. Ströhle leg.; OÖLM.

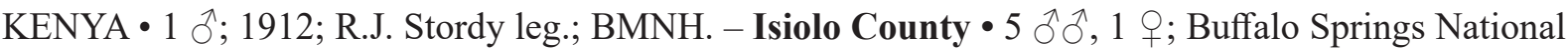
Reserve; [0³1'17.32" N, 37³7'2.53" E]; 15-18 Jul. 1987; H.R. Feijen leg.; RMNH. - Kajiado County • $1 \mathrm{O}^{\top}$; Olorgesailie National Monument; $1.57930^{\circ} \mathrm{S}, 36.44566^{\circ}$ E; 30 Oct.-13 Nov. 2011; R. Copeland

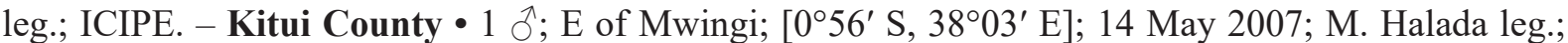

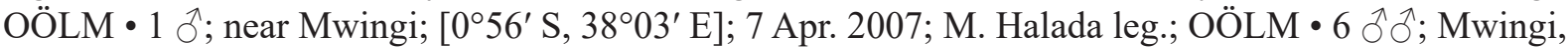
Kangonde vadi; [0 $0^{\circ} 6^{\prime}$ S, 38 $03^{\circ}$ E]; 18 Apr. 2007; M. Halada leg.; OÖLM • 1 ô; Shrubland near Kwandula Hill, Simsi area; $2.01477^{\circ}$ S, 38.32618 E E; 1-4 Dec. 2013; J. Bukhebi and R. Copeland leg.; ICIPE. - Kwale County • 1 क; Shimoni; [4³9' S, 39²3' E]; Dec. 1911; Ch. Alluaud and Jeannel leg.; MNHN. - Laikipia County - 1 क; same collection data as for holotype but 2 Feb. 2005; BMNH • 1 q; same collection data as for preceding; BMNH. - Makueni County • 1 \%; Makindu; [ $2^{\circ} 16^{\prime} 41^{\prime \prime} \mathrm{S}$,

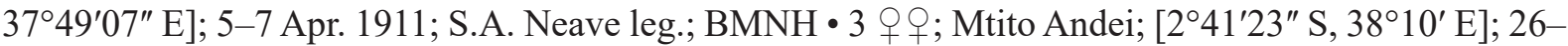
28 Mar. 1911; S.A. Neave leg.; BMNH. - Samburu County 1 d ; 11 mi. S of Mukawa; [1 ${ }^{\circ} 02^{\prime} 28^{\prime \prime}$ N, $36^{\circ} 40^{\prime} 25^{\prime \prime}$ E]; 2 Sep. 1957; CAS. - Taita-Taveta County • 1 q; Mt Kasigau, bottom of forest; $3.82080^{\circ} \mathrm{S}$,

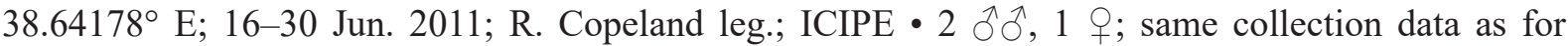

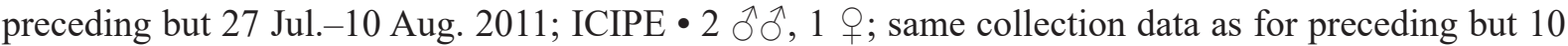

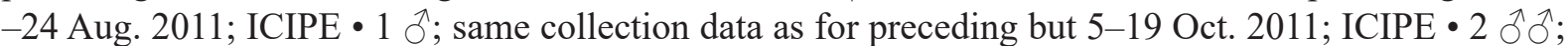

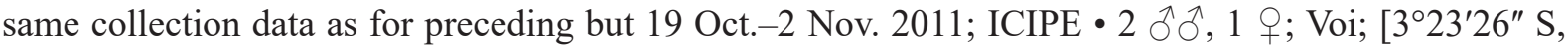

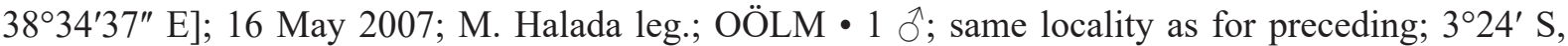

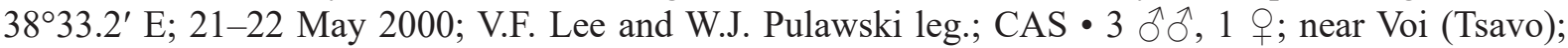
[3²3'26" S, 38³4'37" E]; 8-18 Nov. 1996; Mi. Halada leg.; OÖLM • 1 ô; same collection data as for preceding but 22 Nov. -2 Dec. 1996; OÖLM $\bullet 1 \hat{O}, 1$ o; same locality as for preceding; 23 Mar.4 Apr.1997; Ma. Halada leg.; OÖLM.

SOUTH SUDAN - Bahr el Ghazal Region • 1 \%; "M’Bokou”; 1912; Gailland leg.; MNHN.

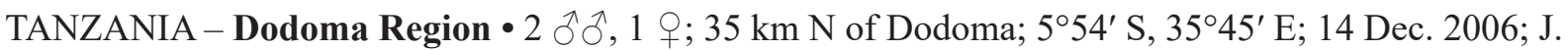
Halada leg.; OÖLM • 1 ' $; 70 \mathrm{~km} \mathrm{~N}$ of Dodoma; $5^{\circ} 40^{\prime} \mathrm{S}, 35^{\circ} 48^{\prime} \mathrm{E}$; 16 Dec. 2006; J. Halada leg.; OÖLM.

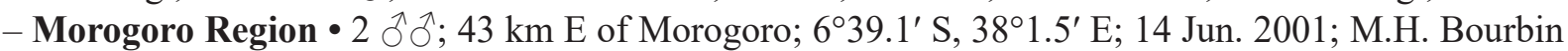

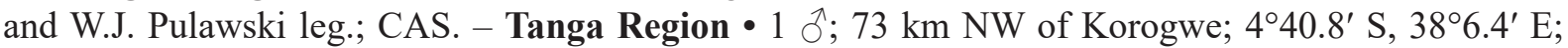
18 Jul. 2001; O.S. Haji and W.J. Pulawski leg.; CAS.

\section{Description}

Female

SIZE. $17.0-21.6 \mathrm{~mm}$.

COLOR. Black except for the following, which are ferruginous: basal half of mandible, lower half of clypeus, scape, pedicel, flagellomeres I-IV, collar, pronotal lobe, prepectus, tegula, legs from coxa onward except inner margin of claw, scutellum, metanotum, metasomal segment I laterally and ventrally, and metasomal segments V and VI. Apical margin of forewing with fuscous spot, cellular area of forewing and entire hindwing hyaline.

VeSTITURE. Appressed setae on clypeus, paraocular area, collar, scutum and propodeal enclosure silvery. Erect setae on clypeus silvery-golden, on paraocular area, collar, scutum and propodeal enclosure silvery. Erect propodeal setae oriented anteriorly. Clypeus without glabrous spot. Scutellum densely and finely pubescent. 
Structure. Free clypeal margin gently notched medially, not stepped above. Clypeus without indentation or carina. Scutellum flat. Metanotum not raised, not bituberculate. $2^{\text {nd }}$ recurrent vein joins markedly proximal from interstitium between submarginal cells II and III. Propodeal enclosure with 4-5 transversal ridges. Foretarsomere I 1.65-1.95 $\times$ length of antepenultimate spine. Petiole length 1.70$1.85 \times$ its medial width.

\section{Male}

SizE. $12.7-18.2 \mathrm{~mm}$.

COLOR. Black except for ferruginous stripe in center of mandible. Wings hyaline, apical margin of forewing fuscous.

VestiTURE. Appressed and erect setae on clypeus, paraocular area, collar, scutum and propodeal enclosure silvery. Erect propodeal setae oriented anteriorly. Clypeus without glabrous spot. Scutellum densely and finely pubescent.

Structure. Free clypeal margin simple, not stepped medially. Scutellum flat. Metanotum not raised, not bituberculate. $2^{\text {nd }}$ recurrent vein joins submarginal cell II medially. Propodeal enclosure with a few extremely indistinct transversal ridges. Posterior margin of metasomal tergum VII convex. Posterior margin of metasomal sternum VII simple, of metasomal sternum VIII triangular. Penis valvae without conspicuous modifications. Petiole length 2.0-2.2 $\times$ its medial width. Flagellomeres IV-VI with narrow placoids covering their proximal halves.

\section{Variation}

Unknown.

\section{Distribution}

Eastern Africa.

$$
\begin{aligned}
& \text { Sphex rufoclypeatus sp. nov. } \\
& \text { urn:Isid:zoobank.org:act:5DBDFA5B-EA8C-45D3-8F41-D6CE0973DF9F }
\end{aligned}
$$

Figs 110-111, 118 (blue)

\section{Differential diagnosis}

Females of this species (Fig. 110) are easy to recognize within the satanas group. In contrast to those of $S$. satanas s. lat. (Figs 113-114) and S. socotrensis sp. nov. (Fig. 116), they have both the scutellum and the metanotum uniformly ferruginous, a character which they share with female $S$. pseudosatanas sp. nov. (Fig. 109). However, S. pseudosatanas sp. nov. has the upper half of the clypeus and the entirety of the scutum black, whereas the clypeus and the anterior half of the scutum are ferruginous in S. rufoclypeatus sp. nov.

Identification of males is more difficult. While male $S$. socotrensis sp. nov. stand out due to their ferruginous legs (Fig. 117), S. satanas satanas (Fig. 115), S. pseudosatanas sp. nov. and S. rufoclypeatus sp. nov. (Fig. 111) all have a black integument and silvery setae. The most reliable characters to distinguish them are a combination of wing infuscation and placoid pattern. The male of $S$. satanas satanas has uniformly fuscous forewings and largely fuscous hindwings, whereas in S. pseudosatanas sp. nov., only the apex of the forewing is fuscous. Sphex rufoclypeatus sp. nov. has slightly infuscate forewings and hyaline hindwings. Sphex satanas satanas and $S$. pseudosatanas sp. nov. share the pattern of narrow placoids on the proximal half or third of flagellomeres IV-VI, whereas S. rufoclypeatus sp. nov. has broad placoids covering the entire length of flagellomeres III-VI. 


\section{Etymology}

'Rufoclypeatus' is a Latin participle meaning 'armed with a red shield,' referring to the ferruginous color of the female clypeus.

\section{Material examined}

\section{Holotype}

CENTRAL AFRICAN REPUBLIC - Bamingui-Bangoran - + ; Koukourou Bamingui Reserve; $7^{\circ} 15^{\prime}$ N, 2003' E; 20 Apr. 2010; J. Halada leg.; THD-012-OOLM; GenBank CO1 gene: MW538570; OÖLM.

\section{Paratypes}

BENIN • 1 क; "Cercle de Djougou-Kouandé”; [1003'45.5" N, 140'40.9" E]; 1908; Brot leg.; MNHN.

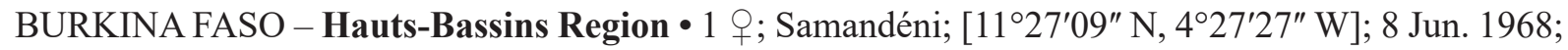
J. Hamon leg.; MNHN.

CENTRAL AFRICAN REPUBLIC - Ouham-Pendé • 1 q; Bozoum; [6 $6^{\circ} 19^{\prime} 02^{\prime \prime}$ N, 16²2'42" E]; 1-10 May 1914; G. Tessmann leg.; ZMB.

GHANA - Northern Region • 1 q; Malawe [Maluwe]; [840'17" N, $2^{\circ} 17^{\prime} 40^{\prime \prime}$ W]; 20 May 1913; J.J. Simpson leg.; BMNH.

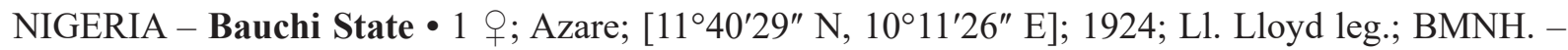
Niger State • 1 $^{\text {; }}$; Zungeru; [948'26" N, 609'08" E]; Nov. 1911; J.W. Scott Macfie leg.; BMNH.

SENEGAL • 1 क; between Tambacounda and Kaffrine; 27-30 Dec. 1967; M. Coruet leg.; MNHN.

\section{Description}

\section{Female}

Size. $17.0-21.6 \mathrm{~mm}$.

COLOR. Black except for the following, which are ferruginous: basal half of mandible, clypeus, scape, pedicel, flagellomeres I-V, collar, pronotum, prepectus, mesopleuron, tegula, legs from coxa onward except inner margin of claw, anterior half of scutum, scutellum, metanotum and apical metasomal segment. Cellular area of forewing increasingly fuscous toward apex, with violet iridescence. Apical margin of fore- and hindwing fuscous. Hindwing hyaline.

VeSTITURE. Appressed setae on clypeus, paraocular area, collar, scutum and propodeal enclosure silvery. Erect setae on clypeus and paraocular area golden-ferruginous, on collar, scutum and propodeal enclosure silvery. Erect propodeal setae short, sparse, oriented anteriorly. Lower center of clypeus glabrous. Scutellum sparsely and finely pubescent.

StRUCTURE. Free clypeal margin simple, stepped medially. Clypeus with slight indentation in lower center, without carina. Scutellum flat. Metanotum not raised, not bituberculate. $2^{\text {nd }}$ recurrent vein joins markedly proximal from interstitium between submarginal cells II and III. Propodeal enclosure with 4-5 transversal ridges. Foretarsomere I $1.55-1.65 \times$ length of antepenultimate spine. Petiole length $1.8 \times$ its medial width.

\section{Male}

Size. $15.7 \mathrm{~mm}$.

CoLOR. Black except for ferruginous stripe in center of mandible. Forewing and apical margin of hindwing slightly infuscate. 
VestiTURE. Appressed and erect setae on clypeus, paraocular area, collar, scutum and propodeal enclosure silvery. Erect propodeal setae oriented anteriorly. Free clypeal margin glabrous. Scutellum densely and finely pubescent.

STRUCTURE. Free clypeal margin folded medially. Scutellum flat. Metanotum not raised, not bituberculate. $2^{\text {nd }}$ recurrent vein joins markedly proximal from interstitium between submarginal cells II and III. Propodeal enclosure with four indistinct transversal ridges. Posterior margin of metasomal tergum VII convex. Posterior margin of metasomal sternum VII simple, of metasomal sternum VIII triangular. Penis valvae without conspicuous modifications. Petiole length $1.8 \times$ its medial width. Flagellomeres III-VI with broad placoids covering their entire length.

\section{Variation}

Unknown.

\section{Distribution}

Western to central Africa.

Sphex satanas satanas Kohl, 1898

Figs 113, 115, 118 (yellow)

Sphex satanas Kohl, 1898: 339, ㅇ, §ิ.

Sphex gorgon Kohl, 1913: 204, ․ Synonymized with Sphex satanas by Leclercq 1955: 7.

\section{Differential diagnosis}

Among the members of its species group, the female of $S$. satanas s. lat. can easily be recognized by having an entirely black scutellum and metanotum (Figs 113-114). In S. pseudosatanas sp. nov. (Fig. 109) and S. rufoclypeatus sp. nov. (Fig. 110), these areas are completely ferruginous instead, and $S$. socotrensis sp. nov. has the metanotum and the posterior margin of the scutellum ferruginous (Fig. 116). The subspecies S. satanas memnon subsp. nov. has the propodeal setae black instead of silvery (Fig. 114).

Male specimens are slightly harder to distinguish. They are characterized though their somewhat infuscate cellular wing area (Fig. 115), whereas the other members of the group have it barely fuscous or completely hyaline. Furthermore, males of $S$. satanas possess narrow placoids on the proximal halves of flagellomeres IV-VI, a configuration similar to that of S. socotrensis sp. nov. and S. pseudosatanas sp. nov. However, these two species lack the more or less distinct folding just above the middle of the free clypeal margin, which is present in S. satanas and S. rufoclypeatus sp. nov. (compare Fig. 28).

\section{Material examined}

Lectotype (designated here)

DEMOCRATIC REPUBLIC OF THE CONGO - Kongo Central • P; Boma Sundi; P. Rolin leg.; IRSN.

Presumed paralectotypes (see Remarks)

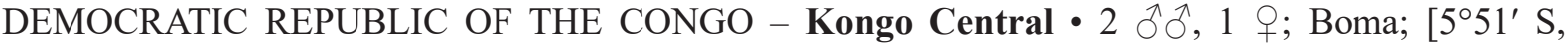

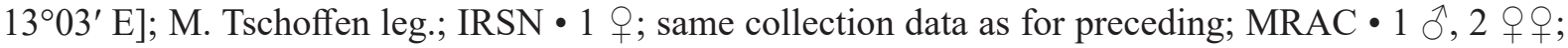

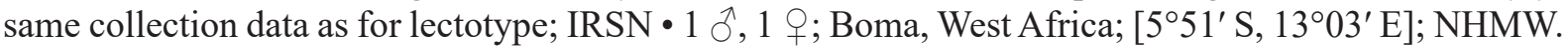

Other material

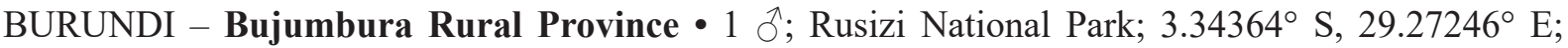
13-20 Apr. 2010; R. Copeland leg.; ICIPE. 
DEMOCRATIC REPUBLIC OF THE CONGO • 2 우; Lulua; 1929; Walker leg.; MRAC. - Haut-

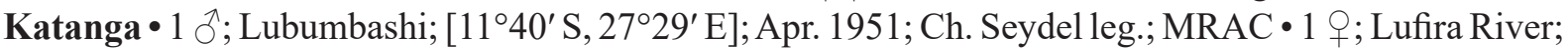

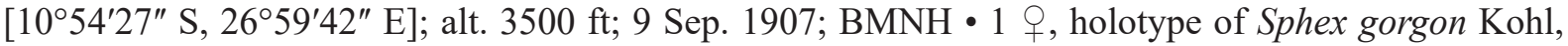
1913; Lukafu; S.A. Neave leg.; MRAC. - Haut-Lomami • 2 q $O$; Tshibamba; [8¹5'42" S, 2421'45" E]; Mar. 1933; F.G. Overlaet leg.; MRAC. - Haut-Uele • 1 ô; "Yebo Moto"; 1926; L. Burgeon leg.; MRAC• 1 q; Vankerhovenville; [ $3^{\circ} 18^{\prime} \mathrm{N}, 2^{\circ} 26^{\prime} \mathrm{E}$ ]; J. Bequaert leg.; MRAC. - Ituri Province • 1 q; Terr. Wamba, Epulu; [1 ${ }^{\circ} 23^{\prime} 52^{\prime \prime}$ N, 2834'51" E]; Sep. 1956; M. Poll leg.; MRAC. - Kasaï-Central • 4 우;

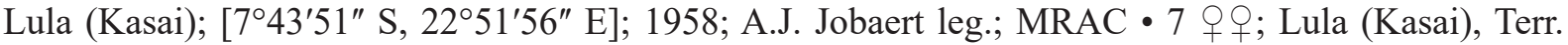

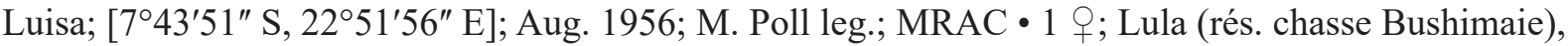
Terr. Luisa; [743'51" S, 22॰51'56" E]; Aug. 1956; M. Poll leg.; MRAC. - Kongo Central • 1 O; Boma;

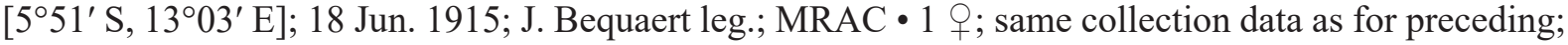
MRAC. - Lomami • 1 q; Lomami, Kambaye; [652'38" S, 234'09" E]; Sep. 1930; P. Quarré leg.; MRAC. - Lualaba • 1 क; 150-200 mi. W of Kambove; 12 Oct. 1904; BMNH • 1 \%; same collection

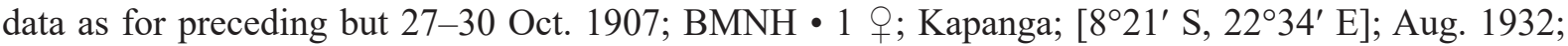
F.G. Overlaet leg.; MRAC • 3 क $\%$; same collection data as for preceding but Sep. 1932; MRAC • 12 우; same collection data as for preceding but Oct. 1932; MRAC • 6 우; same collection data as for preceding but Nov. 1932; MRAC • 8 क 9 ; same collection data as for preceding but Dec. 1932;

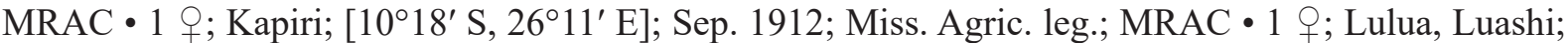

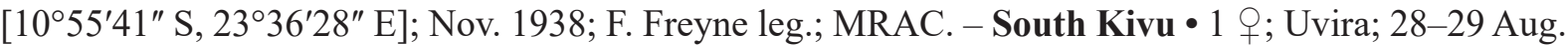
1931; A. Mackie leg.; BMNH. - Tanganyika • 1 ó; Kabalo; [603' S, 2655' E]; 7 Jul. 1947; M. Poll leg.; MRAC.

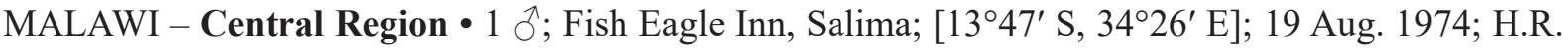

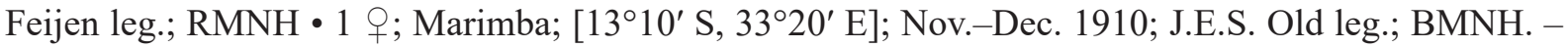

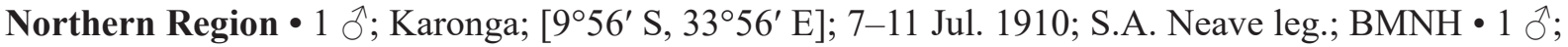

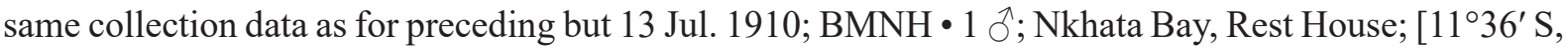

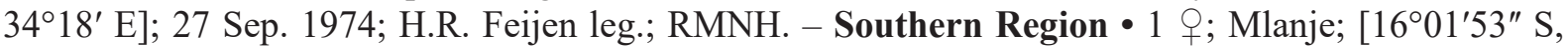
$35^{\circ} 30^{\prime} \mathrm{E}$ ]; 12 Jul. 1913; S.A. Neave leg.; BMNH • 3 우; same collection data as for preceding but 1 Oct. 1913; BMNH • 1 \%; same collection data as for preceding but 8 Oct. 1913; BMNH 1 \% ; same collection data as for preceding but 1 Nov. 1913; BMNH • 1 \%; same collection data as for preceding but 1 Dec. 1913; BMNH • 1 क ; same collection data as for preceding but 21 Dec. 1913; BMNH 1 O ; Zomba; [15²3'09" S, 35¹9'07" E]; Nov. 1913; H.S. Stannus leg.; BMNH.

MOZAMBIQUE - Cabo Delgado Province - 1 क; Kitaragio [near Pangane]; [12 $00^{\prime} 3.4^{\prime \prime}$ S, $\left.40^{\circ} 31^{\prime} 08.5^{\prime \prime} \mathrm{E}\right]$; 19-27 Nov. 2009; O. Montreuil leg.; MNHN • 1 क; Nhica, camp; $10^{\circ} 45.35^{\prime} \mathrm{S}, 40^{\circ} 136^{\prime} \mathrm{E}$; 20 Nov. 2009; C. Villemant leg.; MNHN • 1 q; Vamizi Island; $11^{\circ} 1.97^{\prime}$ S, $40^{\circ} 38.74^{\prime}$ E; C. Villemant leg.; MNHN. - Sofala Province • 1 क; Foret d'Inhandconde; Oct. 1907; G. Vasse leg.; MNHN • 1 q; Tendo du Sungoué, Gorongoza; [18 ${ }^{\circ} 30^{\prime}$ S, 34 $4^{\circ} 03^{\prime}$ E]; Oct. 1907; G. Vasse leg.; MNHN. - Tete Province •

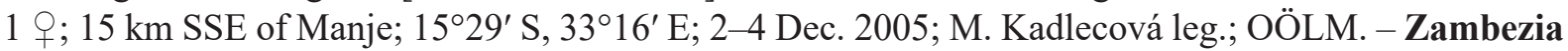

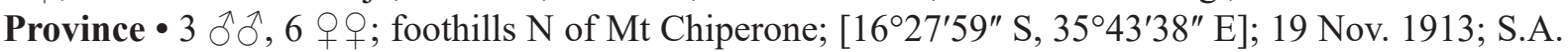
Neave leg.; BMNH • 1 q; same collection data as for preceding but 21 Nov. 1913; BMNH.

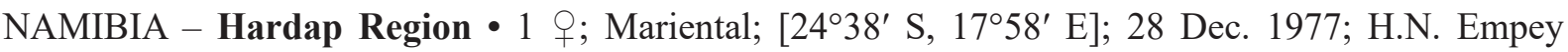

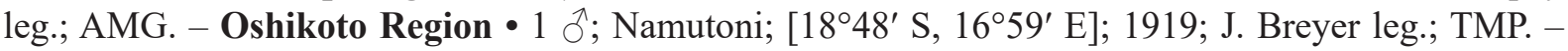

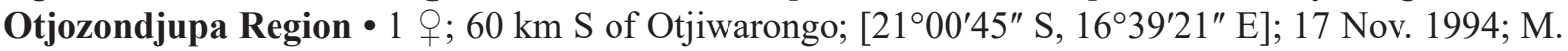

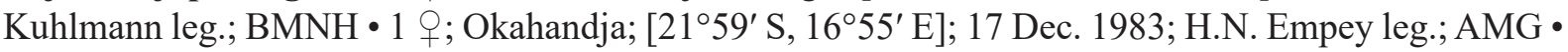
1 q; same locality as for preceding; 3-11 Dec. 1927; R.E. Turner leg.; BMNH • 1 q; same collection data as for preceding but 12-18 Dec. 1927; BMNH • $1{ }^{\top}, 6$ q $\circ$; Waterberg; [ $20^{\circ} 30^{\prime} \mathrm{S}, 17^{\circ} 14^{\prime} \mathrm{E}$ ]; $15-17$ Jan. 1993; F. Koch leg.; ZMB - 1 क; same collection data as for preceding but 31 Oct.-2 Nov. 2007;

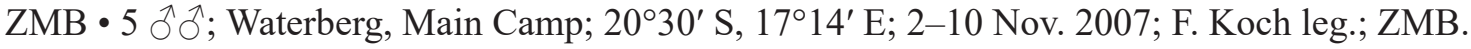




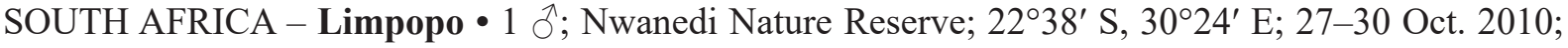
F. Koch leg.; ZMB • 1 क; Sikororo [Ga-Sekororo]; [24²'27.6" S, 30²4'33.3" E]; Jul. 1922; G.P.F. v. Dam leg.; TMP.

TANZANIA • 1 q; Nwaya, N shore of Lake Nyasa; $15-16$ Nov. 1910; S.A. Neave leg.; BMNH • 1 ; Usangu Dist., foot of Kifulufulu Mts; 16-17 Dec. 1910; S.A. Neave leg.; BMNH. - Dodoma Region • 1 ; $50 \mathrm{~km} \mathrm{~N}$ of Kondoa; 4³6' S, 3546' E; 9 Dec. 2006; J. Halada leg.; OÖLM. - Mbeya Region •

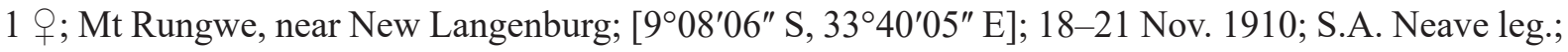
BMNH. - Morogoro Region • 6 ㅇ $ᄋ$; road to Kilossa, Usagara District; [650' S, 36 59' E]; 22-26 Dec. 1910; S.A. Neave leg.; BMNH. - Unguja South Region • 1 q; Zanzibar, Jambiani; [6¹9' S, 39³3' E]; 23 Mar. 1993; K.M. Guichard leg.; BMNH.

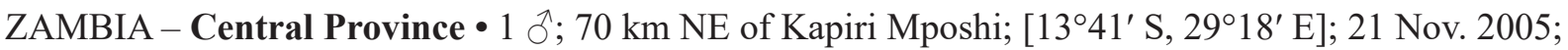
M. Snižek leg.; OÖLM. - Eastern Province • 1 Oૈ, 1 o; "Mid-Luangwa Valley"; 14-16 Aug. 1900; S.A. Neave leg.; BMNH. - Northern Province • 1 + ; Abercorn [now Mbala]; [850' S, 31 ${ }^{\circ} 28^{\prime}$ E]; Oct. 1943; IRSN • 1 क; same collection data as for preceding but Nov. 1943; IRSN • 1 \%; Lake Mweru Wantipa;

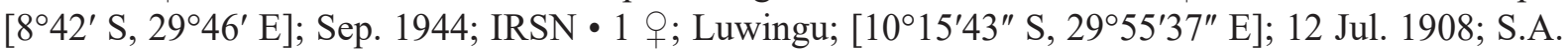

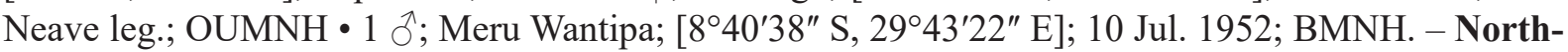
Western Province • 1 क ; Ntambu $150 \mathrm{~km} \mathrm{~W}$ of Solwezi; $12^{\circ} 18^{\prime} \mathrm{S}, 25^{\circ} 10^{\prime} \mathrm{E}$; 11 Nov. 2005; M. Halada leg.; THD-018-OOLM; GenBank CO1 gene: MW538568; OÖLM.

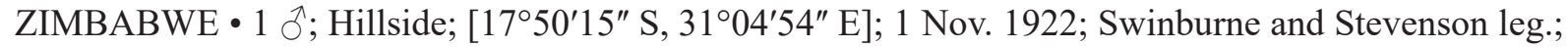

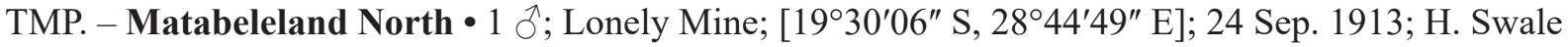
leg.; BMNH • 1 Oे, 1 क; same collection data as for preceding but 25 Sep. 1913; BMNH • 1 क; same collection data as for preceding but 25 Oct. 1914; BMNH • 1 o ; same collection data as for preceding but 2 Jan. 1915; BMNH • 1 क; same locality as for preceding; 9 Feb. 1919; G. Arnold leg.; MNHN • 1 q; Turk Mine; [1944' S, 28 45' E]; 1 Dec. 1958; AMG. - Midlands • 1 क; Shangani, $60 \mathrm{~km}$ SW of Gweru; [1946' S, 29²2' E]; 2 Dec. 1998; Ma. Halada leg.; OÖLM.

\section{Description}

Female

Size. $18.6-23.0 \mathrm{~mm}$.

CoLOR. Black except for the following, which are ferruginous: basal half of mandible, clypeus, scape, pedicel, flagellomeres I-III, collar, pronotal lobe, tegula, prepectus, and legs except coxa and inner margin of claw. Wings fuscous, with violet iridescence.

VESTITURE. Appressed setae on clypeus, paraocular area, collar, scutum and propodeal enclosure silvery. Erect setae on clypeus, paraocular area, collar and scutum dark ferruginous, on propodeal enclosure silvery. Erect propodeal setae oriented anteriorly. Clypeus without glabrous spot. Scutellum sparsely and finely pubescent.

StRUCTURE. Free clypeal margin simple, stepped medially. Clypeus with indentation in lower center, without carina. Scutellum flat. Metanotum not raised, not bituberculate. $2^{\text {nd }}$ recurrent vein joins markedly proximal from interstitium between submarginal cells II and III. Propodeal enclosure with 4-6 transversal ridges. Foretarsomere I 1.8-2.1 $\times$ length of antepenultimate spine. Petiole length $1.5-1.6 \times$ its medial width.

Male

Size. 17.3-22.0 mm. 

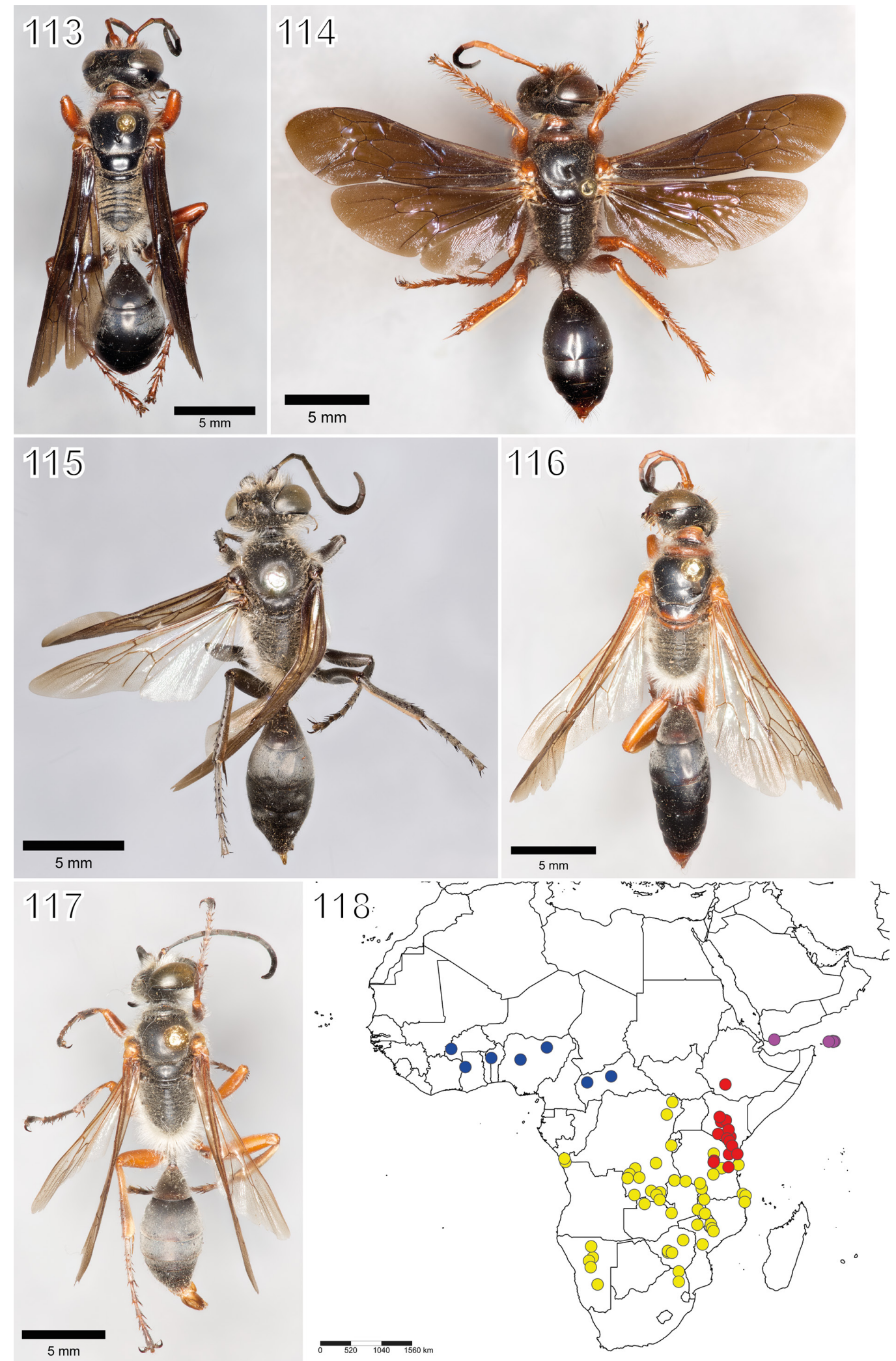

Figs 113-118. 113-114, 116. Habitus of $q$. 115, 117. Habitus of $\widehat{\partial}$. 113, 115. Sphex satanas satanas Kohl, 1898. 114. S. satanas memnon subsp. nov. 116-117. S. socotrensis sp. nov. 118. Geographic distribution of S. pseudosatanas sp. nov. (red), S. rufoclypeatus sp. nov. (blue), S. s. satanas (yellow) and $S$. socotrensis sp. nov. (purple). 
CoLOR. Black except for ferruginous stripe in center of mandible. Forewing fuscous, with violet iridescence. Hindwing largely hyaline.

VeSTITURE. Appressed and erect setae on clypeus, paraocular area, collar, scutum and propodeal enclosure silvery. Erect propodeal setae oriented anteriorly. Clypeus without glabrous spot. Scutellum densely and finely pubescent.

STRUCTURE. Free clypeal margin simple, slightly stepped medially. Scutellum flat. Metanotum not raised, not bituberculate. $2^{\text {nd }}$ recurrent vein joins markedly proximal from interstitium between submarginal cells II and III. Propodeal enclosure with 4-6 transversal ridges. Posterior margin of metasomal tergum VII convex. Posterior margin of metasomal sternum VII simple, of metasomal sternum VIII triangular. Penis valvae without conspicuous modifications. Petiole length $1.6-1.9 \times$ its medial width. Flagellomeres IVVI with narrow placoids covering their proximal halves.

\section{Variation}

Unknown.

\section{Distribution}

Central, southwestern and southeastern Africa.

\section{Remarks}

The original description states that the type series consists of five females and three males collected in Boma and housed in the Royal Belgian Museum in Brussels. We were able to locate a matching number of specimens, the locality labels of which read "Boma" or "Boma Sundi", that had been distributed between IRSN and MRAC. Some of them had already been labelled as paratypes by unknown revisers or collection staff, but since these designations were apparently never published, they are not valid. Also, there are a male and a female specimen from Boma bearing type labels by Kohl in the NHMW collection. Which of the aforementioned ten specimens actually constitute the type series cannot be determined with complete certainty, but we feel confident in designating a specimen from the IRSN as the lectotype.

Sphex satanas memnon subsp. nov. urn:lsid:zoobank.org:act:50FDA09F-CC76-4160-B355-CAE7E564D321

Fig. 114

\section{Differential diagnosis}

The female of this subspecies shares the uniformly black scutellum and metanotum of $S$. satanas satanas (Fig. 113) and is overall very similar, with the only difference being that S. s. memnon subsp. nov. has the appressed and erect propodeal setae entirely black (Fig. 114).

\section{Etymology}

'Memnon' is an Ancient Greek noun, used here in apposition, and the name of a mythological Ethiopian king.

\section{Material examined}

Holotype

ETHIOPIA • ; ; “Abbai Gorge”; 23 Oct. 1945; K.M. Guichard leg.; BMNH. 


\section{Description}

Female

Size. $20.6 \mathrm{~mm}$.

CoLOR. Black except for the following, which are ferruginous: basal half of mandible, clypeus, scape, pedicel, flagellomeres I-V, collar, pronotal lobe, tegula, prepectus, and legs except coxa and inner margin of claw. Wings fuscous, with violet iridescence.

VeSTITURE. Appressed setae on clypeus and paraocular area silvery-golden, on collar silvery, on scutum and propodeal enclosure black. Erect setae on clypeus and paraocular area ferruginous, on collar, scutum and propodeal enclosure black. Erect propodeal setae oriented anteriorly. Clypeus without glabrous spot. Scutellum sparsely and finely pubescent.

STRUCTURE. Free clypeal margin gently notched medially, stepped above. Clypeus with slight indentation in lower center, without carina. Scutellum flat. Metanotum not raised, not bituberculate. $2^{\text {nd }}$ recurrent vein joins markedly proximal from interstitium between submarginal cells II and III. Propodeal enclosure with four transversal ridges. Foretarsomere I $1.6 \times$ length of antepenultimate spine. Petiole length $1.2 \times$ its medial width.

\section{Male}

Unknown.

\section{Variation}

Unknown.

\section{Distribution}

Ethiopia.

$$
\begin{gathered}
\text { Sphex socotrensis sp. nov. } \\
\text { urn:lsid:zoobank.org:act:65257FF5-CA69-4417-BA68-92E2FC9885D4 }
\end{gathered}
$$

Figs 116-117, 118 (purple)

\section{Differential diagnosis}

Males of this species are characterized by having at least the trochanter and femur bright ferruginous (Fig. 117), while the legs of males of the other members of the group are uniformly black. Females have the metanotum and the posterior margin of the scutellum ferruginous with the anterior part black (Fig. 116), whereas the scutellum and metanotum are uniformly ferruginous in S. pseudosatanas sp. nov. (Fig. 109) and S. rufoclypeatus sp. nov. (Fig. 110), and uniformly black in S. satanas s. lat. (Figs 113-114).

\section{Etymology}

'Socotrensis' is a Latinization meaning 'from Socotra', as this island is one of the only known locations where the species occurs.

\section{Material examined}

\section{Holotype}

YEMEN - Socotra Governorate - 1 क; Hadibo Plain; alt. 0-300 ft; $\left[12^{\circ} 39^{\prime} \mathrm{N}, 54^{\circ} 01^{\prime} 10^{\prime \prime} \mathrm{E}\right]$; 16 Apr. 1967; K.M. Guichard leg.; BMNH. 
Paratypes

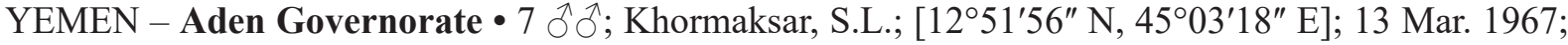
K.M. Guichard leg.; BMNH. - Socotra Governorate - 1 o; same collection data as for holotype; BMNH $\bullet 1$ đ; same locality as for holotype; alt. 0-200 ft; 2 Apr. 1967; K.M. Guichard leg.; BMNH •

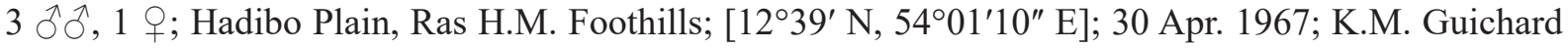

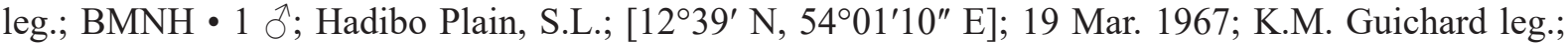
$\mathrm{BMNH} \bullet 8$ ồं same collection data as for preceding but 2 Apr. 1967; BMNH $\bullet 1$; ; same collection data as for preceding but 7 Apr. 1967; BMNH • 1 O ; Hamadara [Hamadero]; [12 ${ }^{\circ} 36^{\prime} \mathrm{N}, 54^{\circ} 16^{\prime} \mathrm{E}$;;

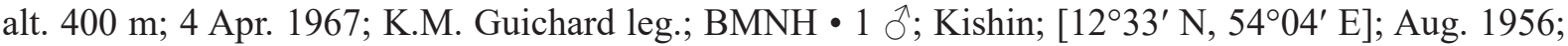
OUMNH exped.; BMNH $\bullet 1$ 万े; same locality as for preceding; alt. 700 m; 18 Apr. 1967; K.M. Guichard

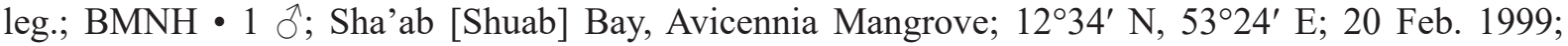
H. Pohl leg.; coll. Schmid-Egger.

\section{Description}

Female

SiZE. 22.1-24.2 $\mathrm{mm}$.

CoLOR. Black except for the following, which are ferruginous: basal half of mandible, clypeus, scape, pedicel, flagellomeres I-IV, collar, apical half of pronotal lobe, tegula, prepectus, posterior two-thirds of scutellum, metanotum and legs excluding apical half of claw. Wings hyaline, apical margin of fore- and hindwing slightly fuscous.

VeSTITURE. Appressed setae on clypeus and paraocular area golden, on collar, scutum and propodeal enclosure silvery. Erect setae on clypeus and paraocular area golden, on collar, scutum and propodeal enclosure silvery. Erect propodeal setae oriented anteriorly. Lower center of clypeus glabrous. Scutellum sparsely and finely pubescent.

StRUCTURE. Free clypeal margin simple, stepped medially. Clypeus without indentation or carina. Scutellum flat. Metanotum not raised, not bituberculate. $2^{\text {nd }}$ recurrent vein joins markedly proximal from interstitium between submarginal cells II and III. Propodeal enclosure with 5-8 transversal ridges. Foretarsomere I 1.7-1.8 $\times$ length of antepenultimate spine. Petiole length $1.3-1.5 \times$ its medial width.

\section{Male}

Size. $15.6-19.3 \mathrm{~mm}$.

CoLOR. Black except for the following, which are ferruginous: basal half of mandible, scape, tegula, legs except coxa and tarsomere V, and metasomal sterna I and VIII. Wings hyaline, apical margin of forewing slightly fuscous.

Vestiture. Appressed and erect setae on clypeus, paraocular area, collar, scutum and propodeal enclosure silvery. Erect propodeal setae oriented anteriorly. Clypeus with small glabrous spot in upper center. Scutellum densely and finely pubescent.

Structure. Free clypeal margin simple. Scutellum flat. Metanotum not raised, not bituberculate. $2^{\text {nd }}$ recurrent vein joins markedly proximal from interstitium between submarginal cells II and III. Propodeal enclosure without any notable ridges. Posterior margin of metasomal tergum VII convex. Posterior margin of metasomal sternum VII simple, of metasomal sternum VIII very slightly concave. Penis valvae without conspicuous modifications. Petiole length $2.2-2.5 \times$ its medial width. Flagellomeres IVVI with narrow placoids covering their proximal two-thirds. 


\section{Variation}

Unknown.

\section{Distribution}

Socotra, southwestern Yemen.

\section{Species of the malagassus group}

The malagassus group contains the following two species: Sphex comorensis sp. nov. and S. malagassus.

The species in this group possess perpendicularly-oriented erect propodeal setae, and the scutellar vestiture is somewhat dense and can be fine or coarse. Therefore, members can be superficially similar to those of the meridionalis- and umtalicus groups. What reliably separates them from these groups is the fact that their metanotum is not raised at all, whereas members of the other two groups normally have it somewhat elevated towards its center, although without the distinct tubercles which are present in the argentatus group. In the malagassus group, the erect propodeal setae are straight instead of curved, and the wings are no more than slightly infuscate. Finally, both species are only known from Madagascar or the surrounding islands, repectively, whereas the members of the meridionalis- and umtalicus group seem to be restricted to the mainland.

$$
\begin{aligned}
& \text { Sphex comorensis sp. nov. } \\
& \text { urn:Isid:zoobank.org:act:79F4CFD2-FBBC-41BD-AD25-4C526C3D01C6 } \\
& \text { Figs 119, } 123 \text { (red) }
\end{aligned}
$$

\section{Differential diagnosis}

Both sexes of this species lack the conspicuous ferruginous stripe which is present laterally on the mesosoma in most specimens of $S$. malagassus. Additionally, males (Fig. 119) are recognizable through having the clypeus almost completely black, in contrast to the entirely ferruginous clypeus of S. malagassus. Furthermore, S. malagassus has the penis valvae modified in a very conspicuous way (Figs 121-122), while those of S. comorensis sp. nov. have the same shape as in most African species of Sphex.

In females of $S$. comorensis sp. nov., the appressed setae on the head and mesosoma are rich golden, whereas those of $S$. malagassus are pale silvery.

\section{Etymology}

'Comorensis' is a Latinization meaning 'from the Comoros', as this archipelago is the only known location where the species occurs.

\section{Material examined}

\section{Holotype}

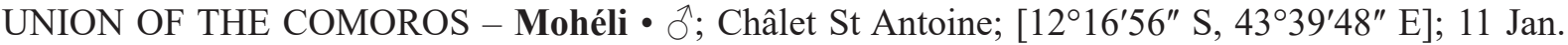
2003; U. Dall'Asta leg.; THD-030-MRAC; GenBank CO1 gene: MW538557; MRAC.

\section{Paratypes}

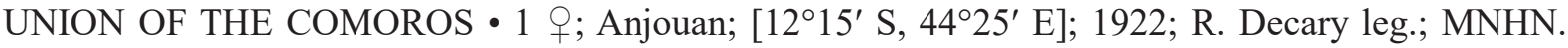

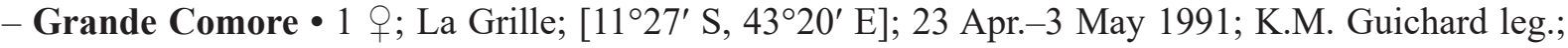

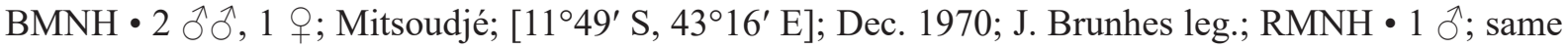
collection data as for preceding; ZMB. 


\section{Description}

\section{Female}

Size. $16.7-22.3 \mathrm{~mm}$.

COLOR. Black except for the following, which are ferruginous: basal half of mandible, labrum, lower half of clypeus, scape, pedicel ventrally, tegula, metasoma laterally, fore- and midfemur apically, foreand midtibia, fore- and midtarsomeres I-IV and basal half of hindtibia. Wings slightly fuscous, basal half with inconspicuous brownish-yellow tinge, with violet iridescence.

VeSTITURE. Appressed setae on clypeus, paraocular area, collar, scutum and propodeal enclosure golden. Erect setae on clypeus and paraocular area ferruginous, on scutum golden intermixed with black ones, on collar and propodeal enclosure golden. Erect propodeal setae oriented perpendicularly, slightly curved towards anterior. Clypeus medially with very narrow vertical glabrous stripe. Scutellum densely and coarsely pubescent.

STRUCTURE. Free clypeal margin with notched process medially, not stepped. Clypeus without indentation, with indistinct longitudinal carina in upper center. Scutellum flat. Metanotum not raised, not bituberculate. $2^{\text {nd }}$ recurrent vein joins interstitium between submarginal cells II and III. Propodeal enclosure without any notable ridges. Foretarsomere I $2.45 \times$ length of antepenultimate spine. Petiole length $1.7-2.0 \times$ its medial width.

\section{Male}

Size. $18.9-21.3 \mathrm{~mm}$.

CoLOR. Black except for the following, which are ferruginous: basal half of mandible, lower center of clypeus, scape, pedicel ventrally, tegula, fore- and midfemur apically, fore- and midtibia, and hindfemor and -tibia ventrally. Cellular wing area hyaline, basal half with inconspicuous brownish-yellow tinge, with violet iridescence, wing apices infuscate.

VeSTITURE. Appressed setae on clypeus, paraocular area, collar, scutum and propodeal enclosure silverygolden. Erect setae on clypeus ferruginous-brown, on paraocular area, collar and propodeal enclosure golden, on scutum golden intermixed with black ones. Erect propodeal setae oriented perpendicularly, slightly curved towards anterior. Clypeus without glabrous spot. Scutellum densely and coarsely pubescent.

STRUCTURE. Free clypeal margin simple. Scutellum flat. Metanotum not raised, not bituberculate. $2^{\text {nd }}$ recurrent vein joins interstitium between submarginal cells II and III. Propodeal enclosure without any notable ridges. Posterior margin of metasomal tergum VII convex. Posterior margin of metasomal sternum VII simple, of metasomal sternum VIII triangular. Penis valvae without conspicuous modifications. Petiole length $2.5-2.8 \times$ its medial width. Flagellomere IV with moderately broad placoid covering only small portions or up to its proximal four-fifths, flagellomere $\mathrm{V}$ with moderately broad placoid covering most of it, flagellomere VI with moderately broad placoid covering only small portions or up to its proximal four-fifths.

\section{Variation}

Unknown.

\section{Distribution}

Comoros. 


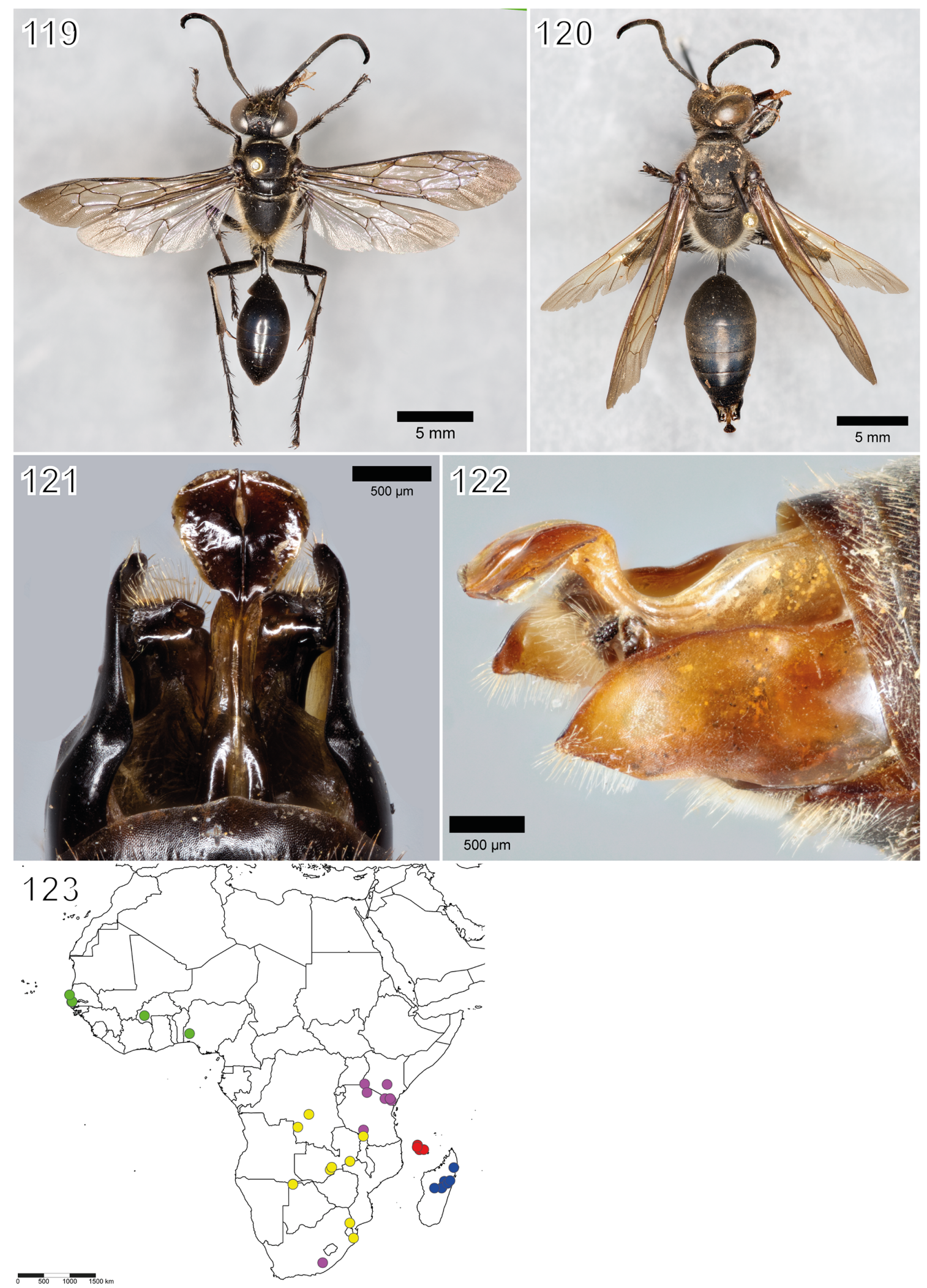

Figs 119-123. 119-120. Habitus of $\widehat{\partial}$. 121. Dorsal view of male genitalia. 122. Lateral view of male genitalia. 119. Sphex comorensis sp. nov. 120-122. S. malagassus de Saussure, 1890. 123. Geographic distribution of $S$. comorensis sp. nov. (red), S. malagassus (blue), S. meridionalis (Arnold, 1947) (yellow), S. nefrens sp. nov. (purple) and S. occidentalis sp. nov. (green). 
Sphex malagassus de Saussure, 1890

Figs 120-122, 123 (blue)

Sphex malagassus de Saussure, 1890: pl. 18 fig. 38, ․

\section{Differential diagnosis}

Males of this species (Fig. 120) can be distinguished from those of S. comorensis sp. nov. (Fig. 119) through their entirely ferruginous clypeus, whereas $S$. comorensis sp. nov. has it black excepting the free margin. Sphex malagassus also has the penis valvae modified in a unique way (Figs 121-122). In females of $S$. malagassus, the appressed clypeal, paraocular and mesosomal setae are inconspicuously pale, while they are brightly golden in those of S. comorensis sp. nov. In addition, most specimens of S. malagassus (but not all) have a broad ferruginous stripe of varying extension laterally on the mesosoma that is absent in $S$. comorensis sp. nov.

\section{Material examined}

MADAGASCAR • 1 क; Hildebrandt leg.; ZMB • 1 đ̊; Staudinger leg.; ZMB. - Alaotra-Mangoro •

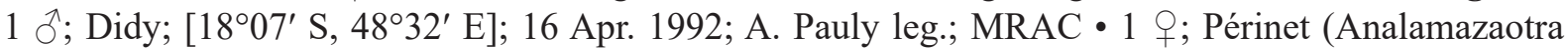
Special Reserve); $18^{\circ} 56^{\prime} \mathrm{S}, 46^{\circ} 16^{\prime}$ E; alt. 950 m; 10 May 1984; R. Hensen and A. Aptroot leg.; RMNH. - Analamanga $\bullet 1 \mathrm{O}^{\top}$; Ambohimanga; $18^{\circ} 45^{\prime} \mathrm{S}, 4^{\circ} 38^{\prime} \mathrm{E}$; alt. $1600 \mathrm{~m} ; 17$ Apr. 1984; R. Hensen and A. Aptroot leg.; THD-022-RMNH; GenBank CO1 gene: MW538555; GenBank LWR gene: MW582285;

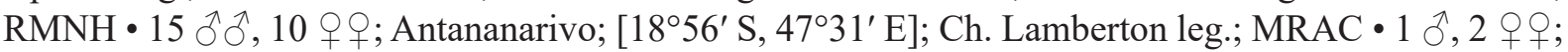

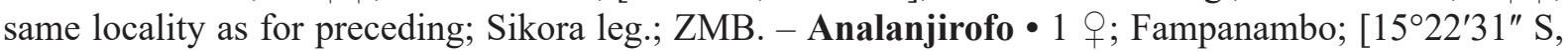
49³7'17" E]; Jun. 1960; J. Vadon leg.; MRAC • 3 우; same collection data as for preceding but Mar.

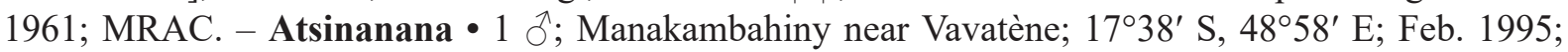

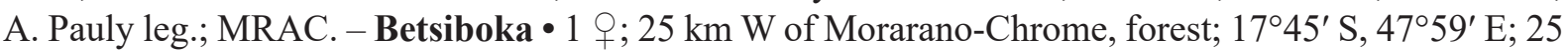
Apr. 1991; A. Pauly leg.; MRAC.

\section{Description}

\section{Female}

SiZE. 22.5-27.9 $\mathrm{mm}$.

CoLOR. Black except for the following, which are ferruginous: basal half of mandible, clypeus, scape, pedicel, outer parts of paraocular area, postgena, neck, collar laterally, tegula and variable lateral stripe from pronotum up to propodeum. Wings hyaline, cellular area with yellow tinge, apex slightly infuscate.

VeSTITURE. Appressed setae on clypeus, paraocular area, collar, scutum and propodeal enclosure silvery-golden. Erect setae on clypeus, paraocular area, collar and scutum black or ferruginous-brown, on propodeal enclosure silvery-golden. Erect propodeal setae oriented perpendicularly or anteriorly. Clypeus without glabrous spot. Scutellum densely and finely pubescent.

StruCtuRE. Free clypeal margin medially notched, not stepped above. Clypeus without indentation and carina. Scutellum convex. Metanotum not raised, not bituberculate. $2^{\text {nd }}$ recurrent vein nearly joins interstitium between submarginal cells II and III. Propodeal enclosure without any notable ridges. Foretarsomere I 2.4-2.5 $\times$ length of antepenultimate spine. Petiole length 1.6-2.1 $\times$ its medial width.

\section{Male}

Size. $21.5-24.5 \mathrm{~mm}$.

CoLOR. Black except for the following, which are ferruginous: basal half of mandible, clypeus, scape and variable lateral stripe from pronotum up to propodeum. Wings hyaline, cellular area with yellow tinge. 
VeSTITURE. Appressed setae on clypeus, paraocular area, collar, scutum and propodeal enclosure silverygolden. Erect setae on clypeus, paraocular area, collar, scutum and propodeal enclosure silvery-golden, occasionally intermixed with black ones. Erect propodeal setae oriented perpendicularly or anteriorly. Clypeus without glabrous spot. Scutellum densely and coarsely pubescent.

StruCtuRE. Free clypeal margin simple. Scutellum convex. Metanotum not raised, not bituberculate. $2^{\text {nd }}$ recurrent vein nearly joins interstitium between submarginal cells II and III. Propodeal enclosure without any notable ridges. Posterior margin of metasomal tergum VII convex. Posterior margin of metasomal sternum VII simple, of metasomal sternum VIII semicircular and slightly pointed. Penis valvae fused, dorsoventrally S-shaped, apically flattened and broadened. Petiole length $1.7-2.1 \times$ its medial width. Flagellomeres IV-VI with broad placoids covering entire their length.

\section{Variation}

Laterally on the mesosoma, the extent of the ferruginous coloration varies.

\section{Distribution}

Madagascar. Several individuals of this species owned by the MRAC were allegedly collected on São Tomé Island, as part of a collecting series by Henri De Saeger from 1932. This locality information appears spurious, since all other records of $S$. malagassus known to us are from Madagascar. It seems unlikely that the species would occur on both these islands when it has never been found anywhere in the area between them. Therefore, we choose to ignore the locality data from the respective specimens.

\section{Species of the meridionalis group}

The meridionalis group contains the following three species: Sphex meridionalis, S. nefrens sp. nov. and S. occidentalis sp. nov.

Members are characterized through their black, more or less perpendicularly-oriented erect propodeal setae which are slightly curved towards the anterior (Figs 3-4), and therefore closely resemble some species of the umtalicus group. Nonetheless, several characters unequivocally separate the two groups. Firstly, females of the meridionalis group (Fig. 8) lack the coarse scutellar vestiture present in those of the umtalicus group (Figs 3-4). As this can be difficult to assess without practice, a useful indicator is the presence or absence of long erect setae, of similar quality to those on the metanotum, near the scutellar center. Females of the meridionalis group only have long erect scutellar setae near the posterolateral margin of the scutellum, whereas those of the umtalicus group usually also have some near the center. Secondly, male specimens of the umtalicus group always have a conspicuous tooth-like process that emerges from below the free clypeal margin (Fig. 27). This structure is absent in members of the meridionalis group.

Sphex meridionalis (Arnold, 1947)

Figs 8, 36, 123 (yellow), 124

Chlorion decipiens meridionalis Arnold, 1947: 145, o (types not examined).

\section{Differential diagnosis}

Females of this species are commonly misidentified as $S$. haemorrhoidalis, since both have clypeus and legs ferruginous, and they are identical in the orientation and coloration of their erect propodeal setae. However, S. haemorrhoidalis, like all members of the umtalicus group, is characterized by their dense and coarse scutellar vestiture (Figs 3-4). On the other hand, the scutellar vestiture in the meridonalis 
group is very fine and often only visible from an angle, and there are very few long erect setae centrally. Female S. meridionalis (Fig. 124) differ from those of S. nefrens sp. nov. (Fig. 125) in having the appressed facial setae ferruginous-golden (Fig. 36) instead of brassy (Fig. 37). Sphex occidentalis sp. nov. has the appressed facial setae in a less ferruginous tone of golden, and its collar, scutellum and metanotum are noticeably ferruginous (Fig. 127).

Male specimens of S. meridionalis and its presumed sister species, S. nefrens sp. nov. (Fig. 126), cannot yet be reliably distinguished based on morphology. They resemble those of S. decipiens (Fig. 100) and are most easily differentiated from that species by having a shorter, wider petiole, a ferruginous clypeal margin and a different placoid pattern. Males of S. occidentalis sp. nov. (Fig. 128) are recognizable through having part of their flagellum, legs and mesosomal dorsum ferruginous.

\section{Material examined}

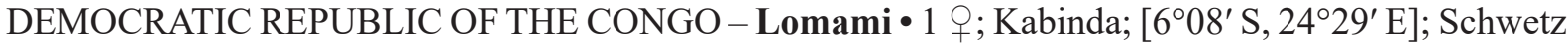
leg.; MRAC. - Lualaba • 1 क; Kapanga; [8²1’ S, 22³4' E]; May 1933; F.G. Overlaet leg.; MRAC.

MALAWI - 1 ; ; NW shore of Lake Nyasa, between Florence Bay and Karonga; 30 Jun.-6 Jul. 1910;

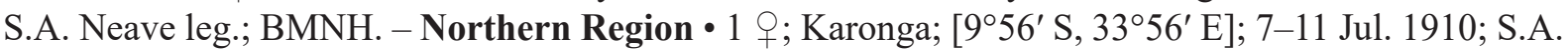
Neave leg.; BMNH • 1 q; same collection data as for preceding but 13 Jul. 1910; BMNH.

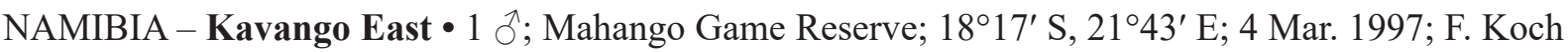
leg.; THD-026-ZMB; GenBank EF-1 $\alpha$ gene: MW558240; GenBank LWR gene: MW582279; ZMB • 1 ; ; same collection data as for preceding; THD-003-ZMB; GenBank CO1 gene: MW538564; ZMB • 1 O; same collection data as for preceding but 2-5 Mar. 1994; ZMB.

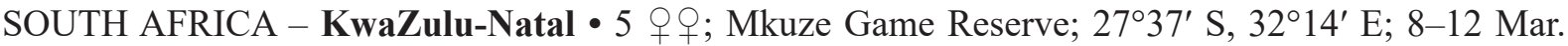
1987; A.J. Weaving leg.; AMG. - Mpumalanga • 1 o; Kruger N. Park, N’Watlinwambu, River-bank Skukuza; [2459'45" S, 31³5'31" E]; 25 Mar. 1998; M. Generani and P.L. Scaramozzino leg.; CAS.

ZAMBIA - Eastern Province • 1 क; "Up. Luangwa Valley”; alt. 1800-2000 ft; $23-24$ Mar. 1908; S.A.

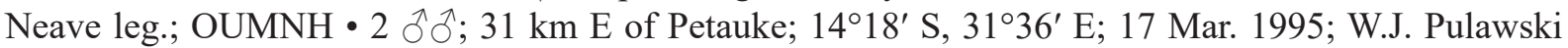

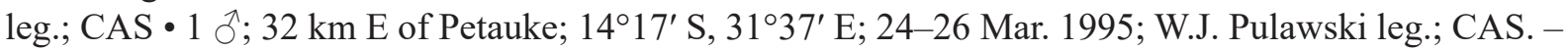

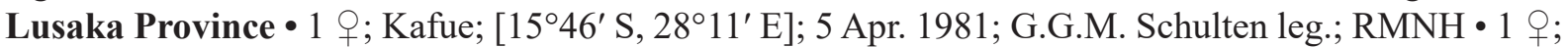

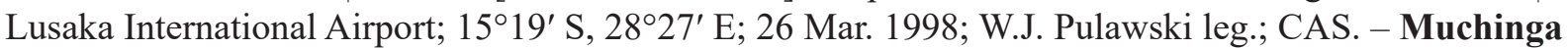
Province • 1 O ; "L. Chambezi V.; Mpika distr."; alt. 3900 ft; 16 May 1908; S.A. Neave leg.; OUMNH • 3 ठ̊ 1 o; same collection data as for preceding but 28 Apr. 1908; OUMNH. - Northern Province $\bullet 1$ 万, 1 ๆ; "L. Chambezi V.; Kasama distr.”; alt. 3900 ft; 3 May 1908; S.A. Neave leg.; OUMNH • 1 q; same collection data as for preceding but 4-6 May 1908; OUMNH • 1 ô, 1 \%; same collection data as for preceding but 9 May 1908; OUMNH 1 ( ${ }^{\top}$; same collection data as for preceding but 14 May 1908; OUMNH.

\section{Description}

Female

SizE. $18.8-25.9 \mathrm{~mm}$.

CoLOR. Black except for the following, which are ferruginous: basal half of mandible, scape, pedicel, flagellomeres I-IV, clypeus, trochanters ventrally and legs from femur onward excepting apical half of claw. Wings fuscous, forewing with violet iridescence.

VESTITURE. Appressed setae on clypeus and paraocular area rich golden, on collar, scutum and propodeal enclosure black. Erect setae on clypeus and paraocular area ferruginous to black, on collar, scutum 


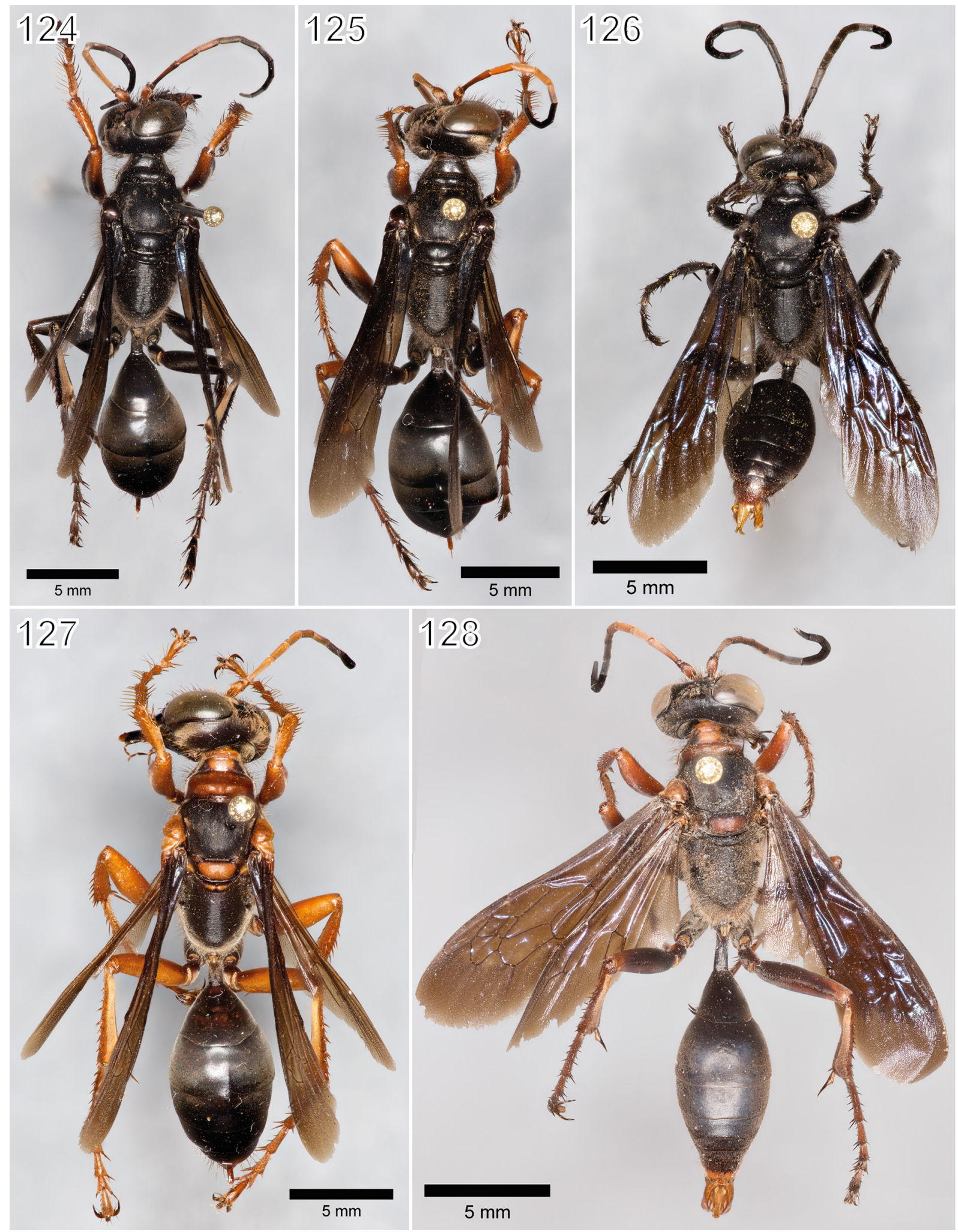

Figs 124-128. 124-125, 127. Habitus of ${ }^{+}$. 126, 128. Habitus of $\widehat{\jmath}$. 124. Sphex meridionalis (Arnold, 1947). 125-126. S. nefrens sp. nov. 127-128. S. occidentalis sp. nov. 
and propodeal enclosure black. Erect propodeal setae oriented perpendicularly, slightly curved towards anterior. Center of clypeus glabrous. Scutellum densely and finely pubescent.

STRUCTURE. Free clypeal margin medially with broad, notched tooth, stepped above. Clypeus with slight indentation in lower center, without carina. Scutellum convex. Metanotum raised, not notably bituberculate. $2^{\text {nd }}$ recurrent vein nearly joins proximal from interstitium between submarginal cells II and III. Propodeal enclosure with numerous very fine transversal ridges. Foretarsomere I 2.2-2.3× length of antepenultimate spine. Petiole length $1.4-1.5 \times$ its medial width.

\section{Male}

Size. $18.4-22.0 \mathrm{~mm}$.

COLOR. Black except for the following, which are ferruginous: basal half of mandible, free clypeal margin medially and apical segment of metasoma. Wings fuscous, with violet iridescence.

VeSTITURE. Appressed setae on clypeus and paraocular area silvery, on collar, scutum and propodeal enclosure black. Erect setae on clypeus, paraocular area, collar, scutum and propodeal enclosure black. Erect propodeal setae oriented perpendicularly, slightly curved towards anterior. Lower center of clypeus glabrous. Scutellum densely and finely pubescent.

STRUCTURE. Free clypeal margin medially concave. Scutellum convex. Metanotum slightly raised, not notably bituberculate. $2^{\text {nd }}$ recurrent vein joins slightly proximal from interstitium between submarginal cells II and III. Propodeal enclosure with numerous very fine transversal ridges. Posterior margin of metasomal tergum VII convex. Posterior margin of metasomal sternum VII simple, of metasomal sternum VIII triangular. Penis valvae without conspicuous modifications. Petiole length $1.6 \times$ its medial width. Flagellomere $\mathrm{V}$ with moderately broad placoid covering almost its entire length.

\section{Variation}

In some females, flagellomeres as well as mid- and hindlegs are largely black.

\section{Distribution}

Central to southeastern Africa.

\section{Remarks}

Despite the fact that we did not examine type material of this species, we are very certain that our interpretation of $S$. meridionalis is correct. Arnold's description is quite helpful and mentions several characters like a ferruginous clypeus, brownish-golden facial setae and markedly infuscate wings. Their combination excludes all other African species of Sphex, and the geographic distribution fits as well.

Sphex nefrens sp. nov.

urn:1sid:zoobank.org:act:9CE718A7-DF94-4037-8413-4147FC20B705

Figs 37, 123 (purple), 125-126

\section{Differential diagnosis}

This species (Figs 125-126) is very similar to S. meridionalis (Fig. 124), as both are characterized by having perpendicularly-oriented and anteriorly-curved erect propodeal setae (compare Fig. 4) combined with a finely pubescent scutellum (Fig. 8). Members of the umtalicus group have the erect propodeal setae oriented the same way, but are distinguished by their more coarsely-pubescent scutellum (Figs 3-4). 
Female members of $S$. meridionalis have rich golden appressed setae on the paraocular area, and their clypeal setae are partially golden as well, but mostly dark (Fig. 36). On the other hand, females of S. nefrens sp. nov. possess uniformly brassy appressed setae on the paraocular region and clypeus (Fig. 37). Female S. decipiens somewhat resemble them in their scutellar vestiture and propodeal sculpture, but have the erect propodeal setae oriented anteriorly and the clypeus almost entirely black, occasionally with a ferruginous margin (Fig. 101), as opposed to a completely ferruginous clypeus in S. meridionalis and in the final member of the meridionalis group, S. occidentalis sp. nov. Sphex occidentalis sp. nov. is distinguished from the other taxa by its ferruginous collar, scutellum and metanotum (Fig. 127).

Male specimens of this species (Fig. 126) and S. meridionalis cannot yet be reliably distinguished based on morphology. They resemble $S$. decipiens (Fig. 100) more than their female counterparts do and are most easily differentiated from that species by the presence of a shorter, wider petiole, appressed golden facial setae and a different placoid pattern. Males of $S$. occidentalis sp. nov. are recognizable through having part of their flagellum, legs and thoracical dorsum ferruginous (Fig. 128).

\section{Etymology}

'Nefrens' is a Latin adjective that translates to 'toothless' and refers to the absence of a tooth-like process on the free clypeal margin in the male of this species, whereas in contrast the process is present in the somewhat similar males of the umtalicus group.

\section{Material examined}

\section{Holotype}

KENYA - Taita-Taveta County • 9 ; Voi; 324' S, 38³3.2' E; 21-22 May 2000; V.F. Lee and W.J. Pulawski leg.; CAS.

\section{Paratypes}

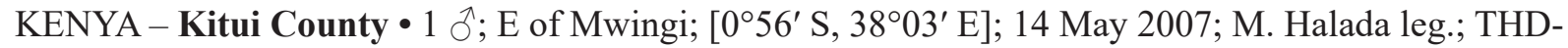
015-OOLM; GenBank CO1 gene: MW538565; GenBank EF-1 $\alpha$ gene: MW558241; GenBank LWR

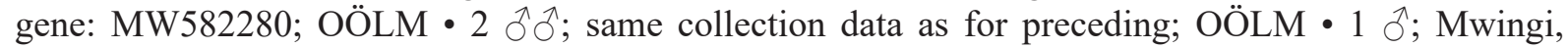
Kangonde vadi; [0 $0^{\circ} 6^{\prime}$ S, 3803' E]; 18 Apr. 2007; M. Halada leg.; OÖLM. - Migori County • 1 ○’; E shore of Victoria Nyanza, near Karungu; [050'35" S, 3409'23" E]; $28-29$ Apr. 1911; S.A. Neave

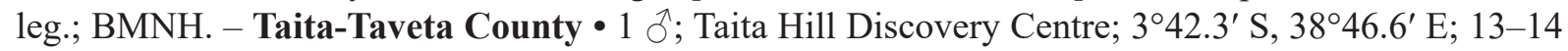

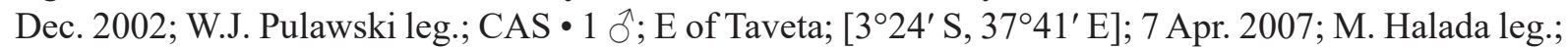

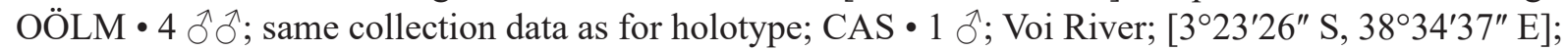
14 Apr. 2007; M. Halada leg.; OÖLM.

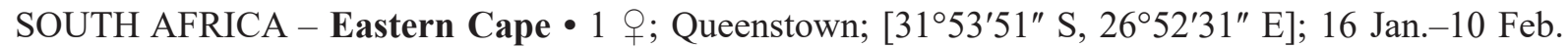
1923; R.E. Turner leg.; BMNH.

TANZANIA • 1 क; Serengeti; [2²0’ S, 34³4' E]; 5 May 2008; A.R.E. Sinclair leg.; ZMB. - Manyara Region • 1 đ̆; "Massai-Nyika; Mgera-Burunge"; Sep. 1893; O. Neumann leg.; ZMB. - Mbeya Region • $1 \mathrm{o}^{\top}$; near Majombe; $8^{\circ} 50^{\prime} 13^{\prime \prime}$ S, 3357'27" E; 29 Feb. 2008; J. Oehlke leg.; coll. Jacobs.

\section{Description}

\section{Female}

Size. $21.5-22.7 \mathrm{~mm}$.

COLOR. Black except for the following, which are ferruginous: basal half of mandible, scape, pedicel, flagellomeres I-IV and basal half of V, clypeus and legs from apical half of femora onward. Wings fuscous, forewing with violet iridescence. 
VeSTITURE. Appressed setae on clypeus and paraocular area brassy, on collar, scutum and propodeal enclosure black. Erect setae on clypeus and paraocular region ferruginous to black, on collar, scutum and propodeal enclosure black. Erect propodeal setae oriented perpendicularly, slightly curved towards anterior. Center of clypeus glabrous. Scutellum densely and finely pubescent.

StRUCTURE. Free clypeal margin medially with broad, notched tooth, stepped above. Clypeus with indentation in lower center, without carina. Scutellum convex. Metanotum raised, not notably bituberculate. $2^{\text {nd }}$ recurrent vein joins markedly proximal from interstitium between submarginal cells II and III. Propodeal enclosure with numerous very fine transversal ridges. Foretarsomere I 3.0-3.2× length of antepenultimate spine. Petiole length $0.9-1.2 \times$ its medial width.

\section{Male}

SIZE. $20.0-21.3 \mathrm{~mm}$.

CoLor. Black except for the following, which are ferruginous: basal half of mandible, free clypeal margin medially and apical segment of metasoma. Wings fuscous, with violet iridescence.

VeSTITURE. Appressed setae on clypeus and paraocular region silvery, on collar, scutum and propodeal enclosure black. Erect setae on clypeus, paraocular region, collar, scutum and propodeal enclosure black. Erect propodeal setae oriented perpendicularly, slightly curved towards anterior. Lower center of clypeus glabrous. Scutellum densely and finely pubescent.

StRUCTURE. Free clypeal margin medially concave. Scutellum convex. Metanotum raised, not notably bituberculate. $2^{\text {nd }}$ recurrent vein joins markedly proximal from interstitium between submarginal cells II and III. Propodeal enclosure with numerous very fine transversal ridges. Posterior margin of metasomal tergum VII convex. Posterior margin of metasomal sternum VII simple, of metasomal sternum VIII triangular. Penis valvae without conspicuous modifications. Petiole length $1.6 \times$ its medial width. Flagellomere $\mathrm{V}$ with narrow placoid covering almost its entire length.

\section{Variation}

Unknown.

\section{Distribution}

Eastern and southern Africa.

Sphex occidentalis sp. nov. urn:1sid:zoobank.org:act:8E9952D5-7732-4DF4-92F3-534DD7774D47

Figs 123 (green), 127-128

\section{Differential diagnosis}

Both sexes of this species are easy to distinguish from other members of the group by having the collar, scutellum and metanotum, or at least their dorsal parts, ferruginous instead of black (Figs 127-128).

\section{Etymology}

'Occidentalis' is a Latin adjective meaning 'western'. It describes the geographic distribution of this species and also references the name of the closely related $S$. meridionalis.

\section{Material examined}

\section{Holotype}

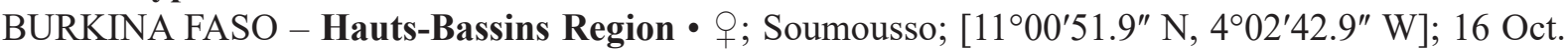
1968; J. Hamon leg.; MNHN. 
Paratypes

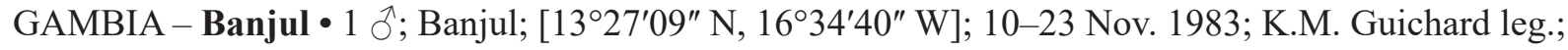
BMNH.

NIGERIA - Oyo State • 2 ${ }^{\circ}$; Fashola near Oyo; [754' N, 347' E]; Nov. 1974; J.T. Medler leg.; $\mathrm{BMNH} \bullet 2$ 우; same collection data as for preceding but 15 Nov. 1975; USNM.

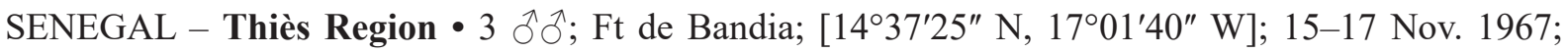
M. Coruet leg.; MNHN.

\section{Description}

Female

Size. 20.1-23.9 mm.

COLOR. Black except for the following, which are ferruginous: basal half of mandible, scape, pedicel, flagellomeres I-V, clypeus, legs from trochanter onward excluding apical half of claw, collar dorsally, tegula, dorsal part of scutellum and metanotum, and apical segment of metasoma. Wings fuscous, forewing with conspicuous purple iridescence.

VeSTITURE. Appressed and erect setae on clypeus, paraocular region, collar and scutum golden, appressed and erect setae on propodeal enclosure brown. Erect propodeal setae oriented perpendicularly, slightly curved towards anterior. Clypeus without glabrous spot. Scutellum densely and finely pubescent.

STRUCTURe. Free clypeal margin slightly stepped medially. Clypeus without indentation and carina. Scutellum convex. Metanotum not raised, not bituberculate. $2^{\text {nd }}$ recurrent vein joins sligthly proximal from interstitium between submarginal cells II and III. Propodeal enclosure with numerous very fine transversal ridges. Foretarsomere I 2.2-2.3 $\times$ length of antepenultimate spine. Petiole length 1.2-1.4× its medial width.

\section{Male}

SizE. 17.1-20.1 mm.

COLOR. Black except for the following, which are ferruginous: basal half of mandible, clypeus excepting dorsolateral area, scape, pedicel, flagellomeres I-V, collar, prepectus, tegula, scutellum and occasionally metanotum dorsally, apical segment of metasoma, legs from trochanter onward excepting trochanters, and femora of mid- and hindlegs dorsally. Wings fuscous, with conspicuous purple iridescence.

VeSTITURE. Appressed setae on clypeus and paraocular area silvery-golden, on collar, scutum and propodeal enclosure ferruginous-brown, on posterior margin of propodeum silvery. Erect setae on clypeus, paraocular region, collar, scutum and propodeal enclosure ferruginous-brown or black, on posterior margin of propodeum silvery. Erect propodeal setae oriented perpendicularly, slightly curved towards anterior. Lower part of clypeus glabrous. Scutellum densely and finely pubescent.

STRUCTURE. Free clypeal margin medially concave, very inconspicuously stepped medially. Scutellum convex. Metanotum raised, not notably bituberculate. $2^{\text {nd }}$ recurrent vein joins slightly proximal from interstitium between submarginal cells II and III. Propodeal enclosure without any notable ridges. Posterior margin of metasomal tergum VII convex. Posterior margin of metasomal sternum VII simple, of metasomal sternum VIII triangular. Penis valvae without conspicuous modifications. Petiole length $1.9 \times$ its medial width. Flagellomere $\mathrm{V}$ with narrow placoid covering almost its entire length.

\section{Variation}

Unknown. 


\section{Distribution}

Western Africa.

\section{Discussion}

1. Phylogenetic relationships between the subgenera of Sphex and the basal species groups

The phylogenetic analyses we conducted based on the sequence data newly generated from the African species and additional data from GenBank (Benson et al. 2013) convincingly indicate the monophyly of Sphex s. str. as a subgenus (Fig. 130). The genus Sphex s. lat., which includes the subgenus Sphex (Fernaldina), has been hypothesized to be paraphyletic (Ohl 1996). We were unable to verify this hypothesis with genetic data, as tree resolution at the generic level was comparatively low in all analyses.

The subgenus newly erected here, Sphex (Menkeella) subgen. nov., is distinguished through having very short, erect propodeal setae and a modified spiracular groove which does not actually connect to the spiracle. Regarding the complete absence of a groove laterally on the propodeum in Fernaldina, it seems reasonable to assume that Menkeella subgen. nov. is more closely related to Sphex s. str. than Fernaldina

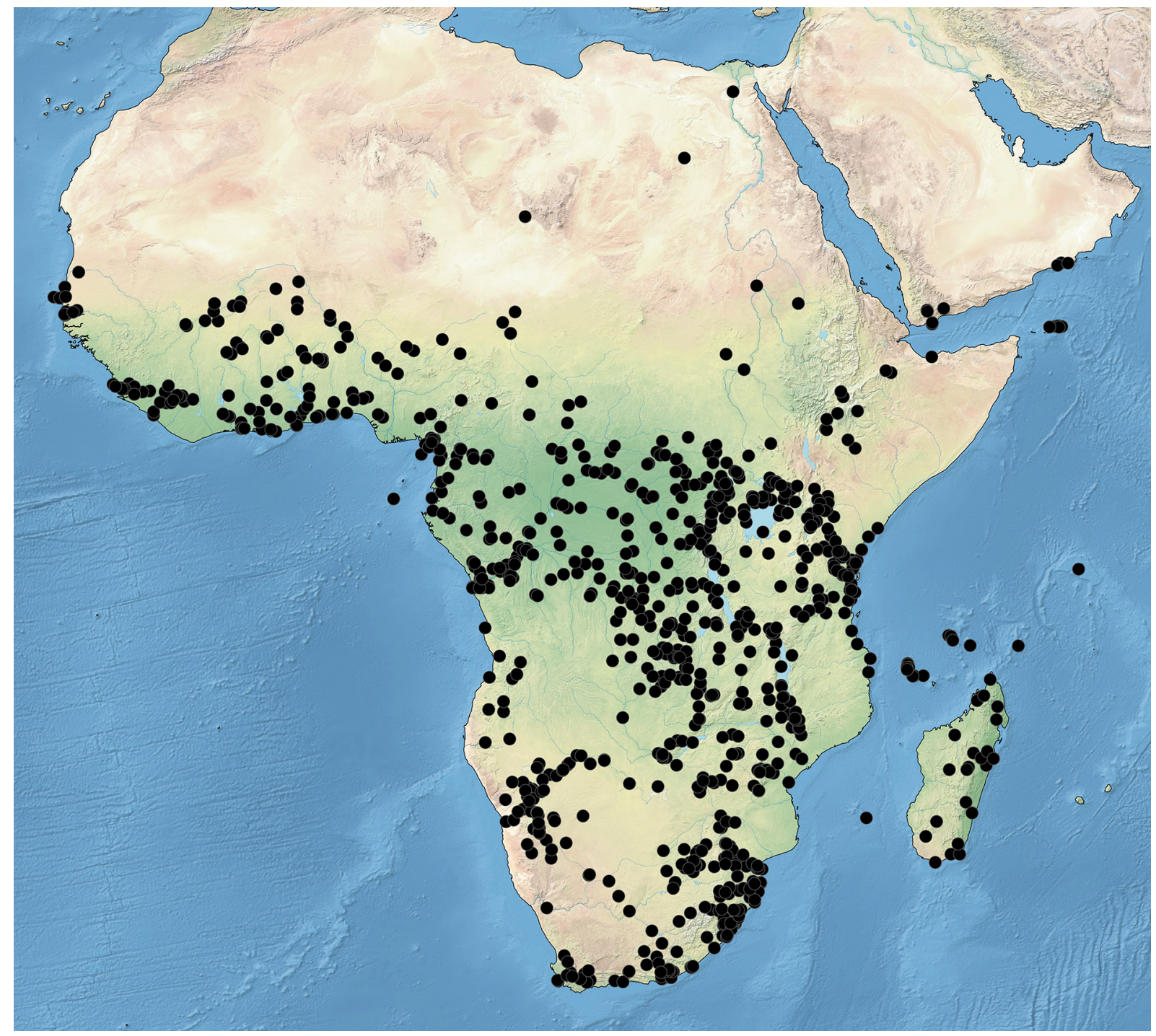

Fig. 129. Geographic coordinates from all examined specimens with traceable localities. 
is. The females of both Menkeella subgen. nov. and Fernaldina, as well as those of Isodontia Patton, 1880 , lack a carina on the labrum, but to our knowledge, that structure is present in all female Sphex s. str. and thus likely an apomorphy of the subgenus Sphex. In conclusion, we presume Sphex (Menkeella) subgen. nov. to be the sister group of Sphex (Sphex) (Fig. 131).

Based on the perpendicular and straight, erect propodeal setae of the malagassus group that greatly resemble those of Isodontia and Fernaldina, this group may be the sister taxon of all other species groups treated here: the remaining Sphex s. str. from the Afrotropical region are apomorphic in having the erect propodeal setae distinctly curved, and usually oriented either anteriorly or posteriorly.

Next, a split presumably occurred between the argentatus group and the remainder of the species. All of its members share a conspicuously bituberculate metanotum as a synapomorphy, and their status as a sister taxon to the other groups is supported by the fact that the argentatus group is the only one encompassing species that have the erect propodeal setae oriented in different ways. Therefore, one would assume that these species did not diverge from their common ancestor as recently as those within the other groups did from theirs. Furthermore, within this group there is a greater amount of interspecific variation regarding the male placoid pattern, as can be seen in Table 2. As the group contains species from the Palearctic, the Oriental and the Australian regions as well (Hensen 1991), the Afrotropical Sphex as a whole appear not to be monophyletic.

The remaining six species groups, all of which are well-defined by morphological and genetic data, can be arranged into three pairs of sister taxa (Fig. 130), with the different orientation of the erect propodeal setae present in each of them being an obvious autapomorphy (Fig. 131). Unfortunately, the evolutionary history among these three supergroups is unclear, as we were unable to find any synapomorphies that would suggest a sister group relationship between any two of them. In the following paragraphs, each supergroup is discussed in further detail.

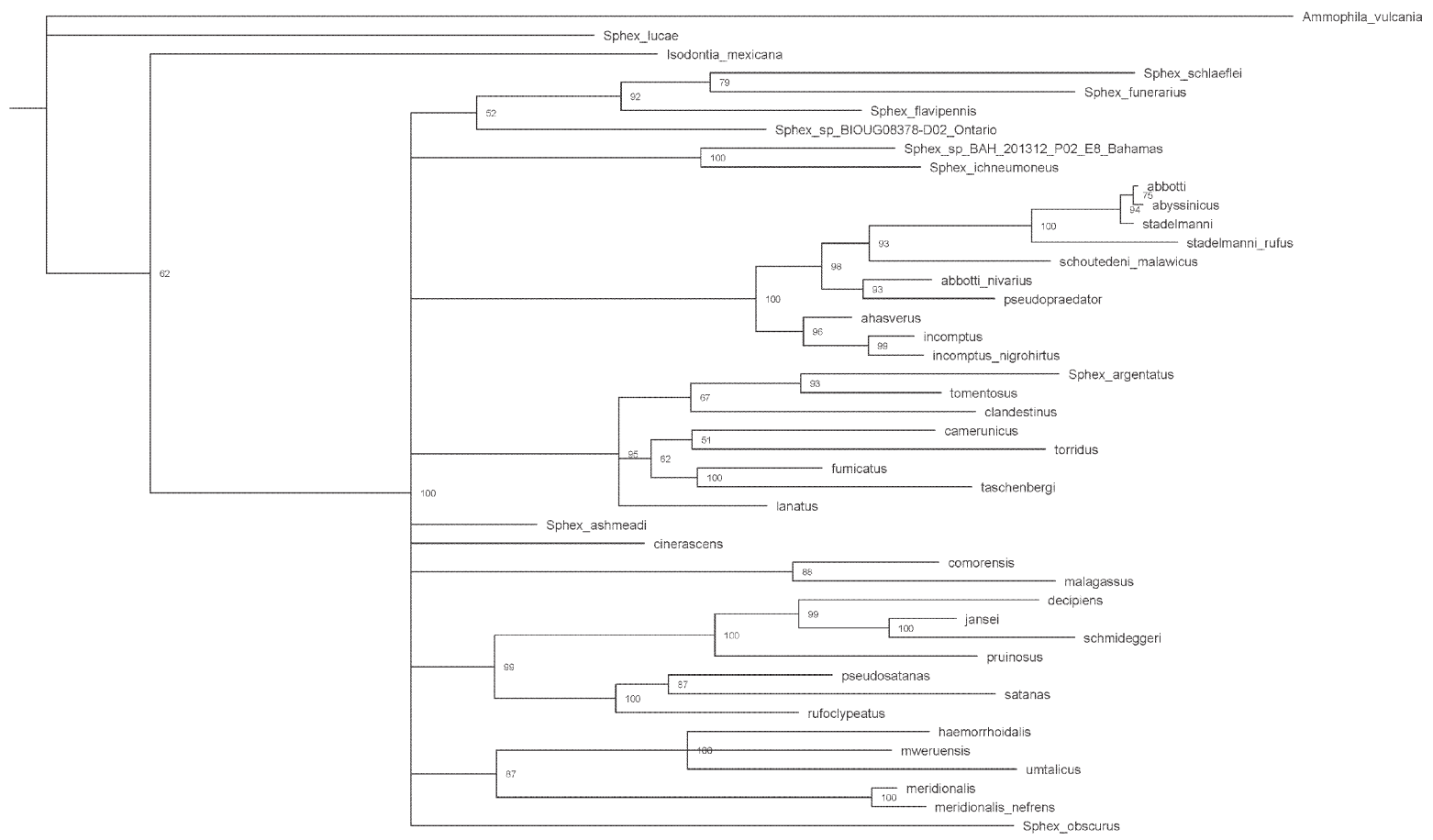

Fig. 130. Bayesian inference phylogenetic tree based on available sequence data of CO1, EF-1 $\alpha$ and LWR, with the posterior probability shown at each node. For the Afrotropical species treated in our study, the genus name has been omitted. 


\section{The bohemanni-nigrohirtus supergroup}

It is very likely that the bohemanni group and the nigrohirtus group are sister taxa. All their members share the posteriorly-oriented erect propodeal setae, an apparently synapomorphic character that is found in almost no other African Sphex, with the exception of S. tomentosus, a member of the argentatus group. Although the erect propodeal setae are also oriented posteriorly in that species, their texture is somewhat different, as they are coarser and less straight, and their density is markedly greater. In this regard, they greatly resemble those of the fellow group member $S$. lanatus, though that species and most others in the argentatus group have them oriented anteriorly. Therefore, we do not think this fact contradicts our hypothesis of posteriorly-oriented erect propodeal setae being an autapomorphy of the bohemanni-nigrohirtus supergroup.

Within this supergroup, $S$. ahasverus and $S$. pseudopraedator sp. nov. are presumably each the sister taxon to the remainder of the species within their respective groups. While the sequence data and some morphological characters suggest that $S$. ahasverus belongs in the nigrohirtus group and S. pseudopraedator sp. nov. in the bohemanni group, several attributes of both species lead us to believe that they might also be assigned conversely. Males of $S$. ahasverus possess the posteriorly somewhat triangular, concavely emarginate sternum VIII that is also found in the other members of the nigrohirtus group, while males of the bohemanni group (with the exception of $S$. pseudopraedator sp. nov.) have sternum VIII more rounded and entire. However, male $S$. ahasverus also have appressed setae covering more than two-thirds of the clypeus, large placoids on flagellomeres IV-VI and, like their female counterparts, a very large and stout body. All of these characters are typical of the bohemanni group. Males of S. pseudopraedator sp. nov. have similarly extensive facial vestiture and an identical placoid configuration in most cases, and females have the appressed facial setae black, which, regarding at least the clypeal center, is also true for all members of the bohemanni group but does not occur within the nigrohirtus group. Nevertheless, both sexes are much more slender and smaller than the other members of the bohemanni group. Additionally, we already mentioned that males deviate in the shape of sternum
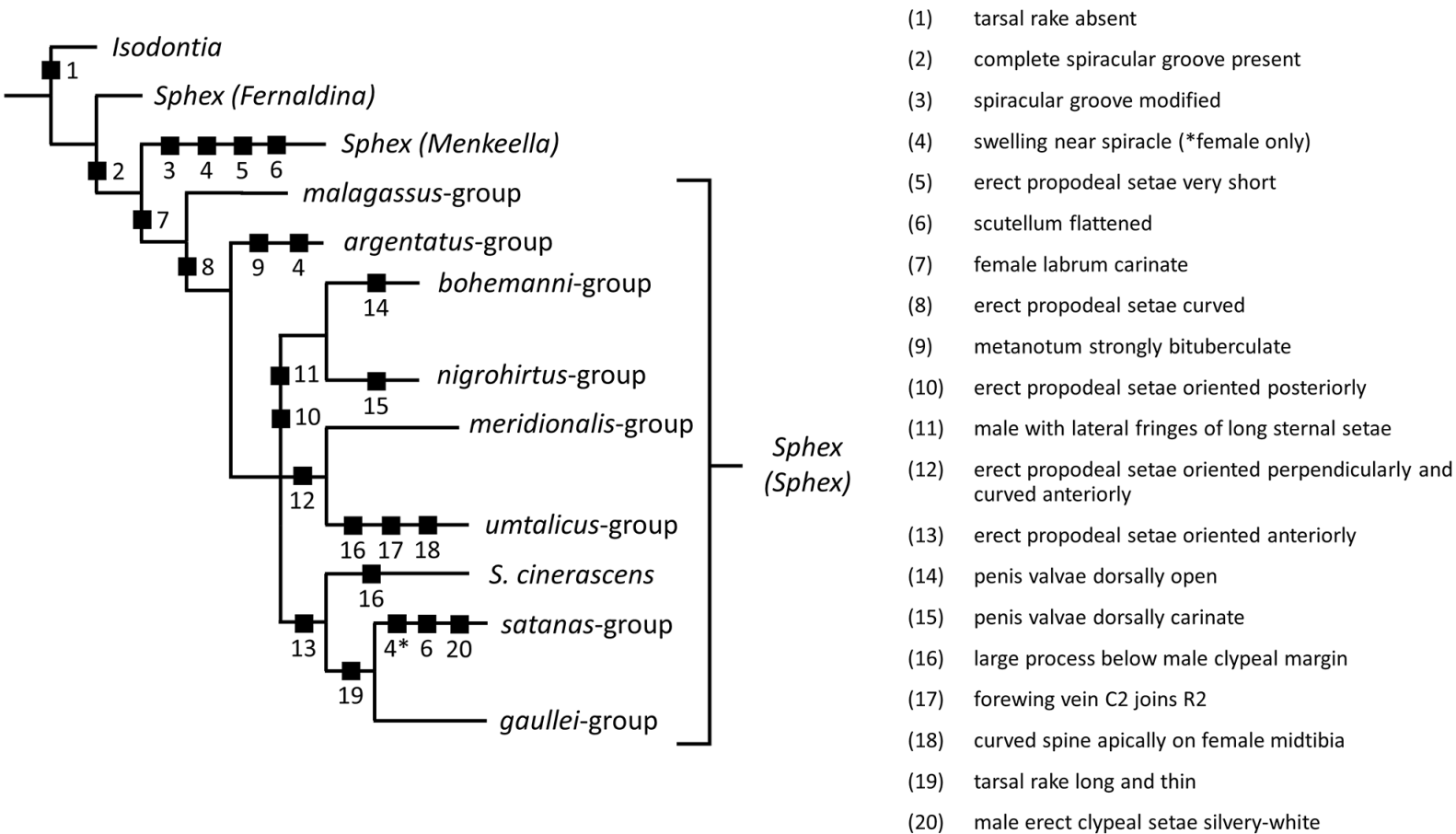

Fig. 131. Preliminary hypothesis on the phylogenetic relationships within Sphex Linnaeus, 1758, based on morphological characters. 
VIII, and the density of the fringes of setae on the sterna notably increases from the base towards sternum VII, as in the males of the nigrohirtus group, whereas those of the bohemanni group often have their density somewhat constant on most sterna.

A conspicuous detail present in the bohemanni group is the remarkable interspecific polymorphism of the male genitalia compared to the other species groups. The combination of characters in penis valvae and sterna VII and VIII are distinct for each species, whereas the remaining groups have the genitalia largely identical even on an inter group-level. A possible explanation for this could lie in the very similar appearance of the females within the bohemanni group. Some of them are barely distinguishable even by experts, so one could speculate that it may also be more difficult for males to find a conspecific mate. If so, evolutionary pressure might have lead to the diversification of male genitalia to prevent accidental cross-species mating. However, this theory is based on the assumption that males use at least some visual cues when searching for a female. Sexual behavior in Sphex has not been extensively analyzed, but studies on $S$. ingens from South America indicate that pheromones excreted by the female are one of the most important aspects in mate finding, as males were observed trying to mate with prey that was left by females (Souza et al. 2015). Therefore, the diverse male genitalia in the bohemanni group are probably the result of other factors.

In any case, the overall low number of diagnostic characters among the members of this group suggests that these species emerged relatively recently. Nonetheless, the few available features are quite diverse and distinct. Based on sex-specific characters like the color of the free clypeal margin for females and the shape of the two apical sterna for males, the members of the bohemanni group can be further assembled into presumed sister taxa. Sphex abbotti s. lat. and S. bohemanni are most likely closely related, as both have a conspicuously notched posterior margin on sternum VII. Furthermore, S. schoutedeni s. lat. and S. stadelmanni s. lat. share a ferruginous clypeal margin and mandibular base in the female, and their penis valvae are structurally very similar. Finally, as detailed above, S. pseudopraedator sp. nov. is probably the sister taxon to the remainder of the group. We cannot reliably place $S$. abyssinicus yet, since its penis valvae and apical sternum are of very unusal shapes, but the presumed respective female greatly resembles that of $S$. abbotti. As discussed before, the molecular data from most species in the group is rather fragmentary, which is probably the reason why the resulting topology of the taxa within the bohemanni group does not fit the morphological data well.

According to the available sequence data, S. camerunicus belongs in the argentatus group, yet we are certain that this placement is incorrect, even though the support values for both the ML and BI analyses are reasonably high. Morphologically, both sexes of S. camerunicus fit extremely well into the nigrohirtus group and do not possess any of the traits that characterize the argentatus group. The presumed error is most likely a result of the data for this species being very sparse, as for the purpose of DNA extraction we only had access to a singular specimen collected after 1980, and DNA amplification proved to be very difficult for that one. As such, we could sequence only about $330 \mathrm{bp}$ of DNA in total.

\section{The gaullei-satanas supergroup}

Another well-supported clade of two sister taxa is the gaullei-satanas supergroup. The members of both groups share anteriorly-oriented erect propodeal setae, and even though this attribute can be found in a number of species of the argentatus group, the character combination which defines that group (conspicuously bituberculate metanotum and swelling anterior of spiracle) is absent in the gaulleisatanas supergroup. Furthermore, the females in the gaullei-satanas supergroup are characterized by a tarsal rake, with spines that are significantly longer and thinner than those of the other African Sphex. We have found that a viable way of quantifying this is the comparison of absolute tarsomere length and absolute spine length, with the exact measuring points being described in the Introduction. While the 
resulting quotient is subject to intraspecific variation, it usually ranges around 1.6 to 1.8 in the gaullei and satanas groups, whereas it is nearly always greater in the remainder of the species (between 1.8 and $3.8)$.

Interestingly enough, some character states in these groups parallel those present within the argentatus group. Members of the gaullei group have faint tubercles on the metanotum, and females in the satanas group possess a swelling near the spiracle. Nonetheless, these traits probably evolved convergently.

There is evidence that the sister taxon to the gaullei-satanas supergroup consists of $S$. cinerascens and its presumed relatives, though of these, only S. cinerascens occurs in Sub-Saharan African. Sphex cinerascens has a potential sister species in S. obscurus (Fabricius, 1793), which is native to southern Asia and the Arabian Peninsula. There seems to be comparatively little congruity in the CO1 region of $S$. obscurus and S. cinerascens, though this could be attributed to the sparsity of available data for S. cinerascens, as both are very similar morphologically. In both species, the anteriorly-oriented erect propodeal setae, characteristic for the gaullei- and satanas groups, are present.

Sphex pruinosus is likely the sister to the remaining members of the gaullei group, as it is differentiated from the other species by its uniformly silvery setae, very long petiole and the lack of a glabrous area on the clypeus. Sphex cinerascens also has a long petiole and a uniformly pubescent clypeus, which corroborates this cladistic placement.

While the three species $S$. jansei, S. gaullei and $S$. schmideggeri sp. nov. are morphologically quite similar, and the details of their phylogenetic relationship are not easily recognizable, $S$. decipiens is clearly their sister taxon. It has the erect setae and almost all of the integument black, as well as the wings conspicuously infuscate, whereas the aforementioned species are all partially ferruginous, with silvery or golden erect setae and largely hyaline wings.

\section{The meridionalis-umtalicus supergroup}

The final supergroup we propose based on morphological and molecular evidence encompasses the meridionalis- and umtalicus group. The erect propodeal setae are oriented perpendicularly in both groups, with a slight curvature toward anterior. Also, paralleling the aforementioned bohemanni-nigrohirtus supergroup, this notable synapomorphic trait apparently evolved convergently at one point within the argentatus group, as $S$. fumicatus and its sister species $S$. taschenbergi have the erect propodeal setae oriented in a similar way, but are otherwise morphologically and genetically clearly distinct.

Van der Vecht (1973) hypothesized that the African S. haemorrhoidalis is part of a species group that also contains at least two species in the Oriental region, one of them being S. subtruncatus Dahlbom, 1843. As diagnostic characters for this group he listed a curved spine at the apex of the midtibia in females, and for males a small transverse lobe that is depressed below the level of the clypeus medially at the anterior clypeal margin. We agree with his assessment, as in addition to these characters, the propodeal sculpture, the orientation of the erect propodeal setae and the density of the scutellar vestiture in S. subtruncatus greatly resemble those of the umtalicus group as well. It represents another argument against the monophyly of the African Sphex, paralleling the case of the argentatus group also encompassing species from other continents.

Based on data from commonly-collected species, there seem to be five major distribution patterns in the Afrotropical region:

1. Sub-Saharan continental area, apparently not restricted to a specific climate zone; a prime example is S. fumicatus (Fig. 48)

2. Southwestern arid and desert regions (S. decipiens, Fig. 112 [red]) 
3. Southeastern and eastern temperate regions (S. umtalicus, Fig. 77 [red])

4. Central and western tropical rainforests (S. camerunicus, Fig. 78 [red])

5. Madagascar and/or the surrounding islands (S. torridus, Fig. 56 [blue])

Naturally, these are very simplified representations, but data is sparse for many species and collectors are very selective in choosing localities, which complicates assessing species distributions in a balanced way over the Afrotropical region.

\section{Acknowledgements}

We would like to thank Frederique Bakker, Terence Bellingan, Seán Brady, Robert Copeland, Wouter Dekoninck, Fritz Gusenleitner, James Hogan, Helen James, Christine LeBeau, John Midgley, Dominic Mogeni, Audrey Ndaba, Wojciech Pulawski, Maria Tavano, Hege Vårdal and Jason Weintraub for arranging the loans, Alexandra Holland, Martin Krüger and Wolfram Mey for helping with the transport and Stéphane Hanot, Andrew Liston, David Notton, Andreas Taeger, Agnièle Touret-Alby, Claire Villemant and Dominique Zimmermann for their friendly supervision during our visits to the respective collections.

Furthermore, we thank Robert Schreiber and Isabelle Waurick for handling the shipment of the laboratory samples and their helpful guidance concerning molecular methodologies, and Christian Schmid-Egger and Xavier Cornejo for providing additional material. We also thank Antonia Göbel and Cora Krautz for testing the identification keys.

Many thanks go to Arnold Menke and Wojciech Pulawski for thoroughly checking the contents, structure and language of the manuscript and improving its quality with their helpful suggestions.

Finally, we thank the state of Berlin for granting the Elsa-Neumann-Scholarship, and the Museum für Naturkunde Berlin, both of which financially supported parts of this study.

\section{References}

Arnold G. 1928. The Sphegidae of South Africa. Part XI. Annals of the Transvaal Museum 12: 338-375.

Arnold G. 1947. New species of African Hymenoptera. No. 7. Occasional Papers of the National Museum of Southern Rhodesia 2 (13): 131-167.

Arnold G. 1951. Sphecidae and Pompilidae (Hymenoptera) collected by Mr. K.M. Guichard in West Africa and Ethiopia. Bulletin of the British Museum (Natural History), Entomology 2: 95-183.

https://doi.org/10.5962/bhl.part.27752

Astrin J.J., Stüben P.E., Misof B., Wägele J.W., Gimnich F., Raupach M.J. \& Ahrens D. 2012. Exploring diversity in cryptorhynchine weevils (Coleoptera) using distance-, character- and tree-based species delineation. Molecular Phylogenetics and Evolution 63 (1): 1-14.

https://doi.org/10.1016/j.ympev.2011.11.018

Bamps P. 1982. Flore d'Afrique centrale (Zaire-Rwanda-Burundi): Repertoire des lieux de récolte. Jardin botanique national de Belgique, Meise, Belgium.

Benson D.A., Cavanaugh M., Clark K., Karsch-Mizrachi I., Lipman D.J., Ostell J. \& Sayers E.W. 2013. GenBank. Nucleic Acids Research 41 (D1): D36-D42. https://doi.org/10.1093/nar/gks1195

Berland L. 1927. Les Sphegidae (Hyménoptères) du Muséum National de Paris ( $4^{\mathrm{e}}$ note). Bulletin du Muséum national d'Histoire naturelle 33: 150-156.

Berland L. 1952. La réserve naturelle integrale du Mt. Nimba. XII. - Hyménoptères vespiformes. Mémoires de l'Institut français d'Afrique noire 19: 271-276. 
Berland L. 1956. Les Sphex africains (Hymenoptera). Bulletin de l'Institut français d'Afrique noire, Série A, Sciences Naturelles 18 (4) : 1161-1181.

Bernstein H., Byerly H.C., Hopf F.A. \& Michod R.E. 1985. Sex and the emergence of species. Journal of Theoretical Biology 117 (4): 665-690. https://doi.org/10.1016/S0022-5193(85)80246-0

Bezdek J., Hula V., Purchart L. \& Kral K. 2012. List of local Socotran geographical names used in entomological literature. Acta Entomologica Musei Nationalis Pragae 52 (Suppl 2): 27-67.

Blaimer B.B., Lloyd M.W., Guillory W.X. \& Brady S.G. 2016. Sequence capture and phylogenetic utility of genomic ultraconserved elements obtained from pinned insect specimens. PLoS One 11 (8): e0161531. https://doi.org/10.1371/journal.pone.0161531

Bohart R.M. \& Menke A.S. 1963. A reclassification of the Sphecinae, with a revision of the Nearctic species of the tribes Sceliphronini and Sphecini. University of California Publications in Entomology 30: 91-182.

Bohart R.M. \& Menke A.S. 1976. Sphecid Wasps of the World: a Generic Revision. University of California Press. https://doi.org/10.1525/9780520309548

Braby M.F., Eastwood R. \& Murray N. 2012. The subspecies concept in butterflies: has its application in taxonomy and conservation biology outlived its usefulness? Biological Journal of the Linnean Society 106: 699-716. https://doi.org/10.1111/j.1095-8312.2012.01909.x

Brauns H. 1917. Notes and synonymy of Hymenoptera in the collection of the Transvaal Museum. Annals of the Transvaal Museum 5 (4): 238-245.

Cameron P. 1908. Hymenoptera, 7. Fossores. In: Sjöstedt Y. (ed.) Wissenschaftliche Ergebnisse der schwedischen zoologischen Expedition nach dem Kilimanjaro, dem Meru und den umgebenden Massaisteppen Deutsch-Ostafrikas 1905-1906. Volume 2 (8-14): 147-296. P. Palmquists Aktiebolag, Stockholm. [cover dated 1910, but page 294 dated Mar. 1908]

Cameron P. 1910. On the aculeate Hymenoptera collected by Mr. A.J.T. Janse, Normal College, Pretoria, in the Transvaal. Annals of the Transvaal Museum 2: 116-154.

Christ J.L. 1791. Naturgeschichte, Klassification und Nomenclatur der Insekten vom Bienen, Wespen und Ameisengeschlecht: als der fünften Klasse fünfte Ordnung des Linneischen Natursystems von den Insekten, Hymenoptera: mit häutigen Flügeln. Volume 1. Hermannischen Buchhandlung, Frankfurt am Main. https://doi.org/10.5962/bhl.title.87724

Dahlbom A.G. 1843-1845. Hymenoptera Europaea praecipue borealia; Formis typicis nonnullis Specierum Generumve exoticorum aut extraneorum propter Nexum systematicus associatis; per Familias, Genera, Species et Varietates disposita atque descripta. Tomus: Sphex in sensu Linneano. Officina Lundbergiana, Lund, Sweden. https://doi.org/10.5962/bhl.title.15890

Danforth B.N., Sauquet H. \& Packer L. 1999. Phylogeny of the bee genus Halictus (Hymenoptera: Halictidae) based on parsimony and likelihood analyses of nuclear EF-1 $\alpha$ sequence data. Molecular Phylogenetics and Evolution 13 (3): 605-618. https://doi.org/10.1006/mpev.1999.0670

Danforth B.N., Brady S.G., Sipes S.D. \& Pearson A. 2004. Single-copy nuclear genes recover Cretaceousage divergences in bees. Systematic Biology 53: 309-326. https://doi.org/10.1080/10635150490423737

De Queiroz K. 2007. Species concepts and species delimitation. Systematic Biology 56 (6): 879-886. https://doi.org/10.1080/10635150701701083

De Saussure H. 1890-1892. Histoire physique, naturelle et politique de Madagascar publiée par Alfred Grandidier. Volume 20. Histoire naturelle des Hyménoptères. Imprimerie Nationale, Paris. 
Dollfuss H. 2008. The Sphecini wasps of the genera Chilosphex Bohart \& Menke, Isodontia Patton, Palmodes Kohl, Prionyx Vander Linden and Sphex Linnaeus of the "Biologiezentrum Linz" collection in Linz, Austria, (Hymenoptera, Apoidea, Sphecidae). Linzer Biologische Beiträge 40: 1399-1434.

Dörfel T.H. \& Ohl M. 2015. A revision of the Australian digger wasps in the genus Sphex (Hymenoptera, Sphecidae). ZooKeys 521: 1-104. https://doi.org/10.3897/zookeys.521.5995

Drury D. 1770-1782. Illustrations of Natural History. Wherein are exhibited upwards of two hundred and forty figures of exotic insects, according to their different genera; very few of which have hitherto been figured by any author, being engraved and coloured from nature, with the greatest accuracy, and under the author's own inspection, on fifty copper-plates. With a particular description of each insect: interspersed with remarks and reflections on the nature and properties of many of them. To which is added, a translation into French. B. White, London.

Fabricius J.C. 1781. Species Insectorum exhibentes eorum Differentias specificas, Synonyma Auctorum, Loca natalia, Metamorphosin adiectis Observationibus, Descriptionibus. Volume 1. C.E. Bohnii, Hamburgi et Kilonii [= Hamburg and Kiel]. https://doi.org/10.5962/bhl.title.36509

Fabricius J.C. 1787. Mantissa Insectorum sistens eorum Species nuper detectas adiectis Characteribus genericis, Differentiis specificis, Emendationibus, Observationibus. Volume 1. C.G. Proft, Hafniae [= Copenhagen]. https://doi.org/10.5962/bhl.title.36471

Fabricius J.C. 1793. Entomologia systematica emendata et aucta. Secundum Classes, Ordines, Genera, Species adjectis Synonymis, Locis, Observationibus, Descriptionibus. 4 volumes. C.G. Proft, Hafniae [= Copenhagen]. https://doi.org/10.5962/bhl.title.122153

Faircloth B.C., Branstetter M.G., White N.D. \& Brady S.G. 2014. Target enrichment of ultraconserved elements from arthropods provides a genomic perspective on relationships among Hymenoptera. Molecular Ecology Resources 15 (3): 489-501. https://doi.org/10.1111/1755-0998.12328

Fernald H.T. 1907. A collection of Sphecidae from Argentine. Bulletin of the Museum of Comparative Zoology 50: 263-272.

Field J., Ohl M. \& Kennedy M. 2011. A molecular phylogeny for digger wasps in the tribe Ammophilini (Hymenoptera, Apoidea, Sphecidae). Systematic Entomology 36 (4): 732-740.

https://doi.org/10.1111/j.1365-3113.2011.00591.x

Fox W.J. 1891. On three species of Hymenoptera from East Africa. Entomological News 2: 42.

Folmer O., Black M., Hoeh W., Lutz R. \& Vrijenhoek R. 1994. DNA primers for amplification of mitochondrial cytochrome c oxidase subunit I from diverse metazoan invertebrates. Molecular Marine Biology and Biotechnology 3: 294-299.

Germar E.F. 1817. Reise nach Dalmatien und in das Gebiet von Ragusa. F.A. Brockhaus, Leipzig and Altenburg, Germany.

Gerstaecker A. 1871. Beitrag zur Insektenfauna von Zanzibar. Archiv für Naturgeschichte 37 (1): 345363.

Gess S.K. \& Gess F.W. 2003. A Catalog for Flower Visiting Records for Aculeate Wasps and Bees in the Semi-Arid to Arid Areas of Southern Africa. Department of Entomology, Albany Museum, Grahamstown, South Africa.

Giordani Soika A. 1942. Sfegidi (Hym.) raccolti nell'Africa orientale dal Prof. Alberto Mochi e dal Dott. Marcello Mochi: 1. - Nuove specie dei generi Sphex e Cerceris. Atti della Società italiana di Scienze naturali e del Museo civico di Storia naturale di Milano 81: 196-209. 
Gribodo G. 1879. Note imenotterologiche. Annali del Museo civico di Storia naturale di Genova 14: 325-347.

Gribodo G. 1894. II. Aculeati e Chrisidi. In: Emery C., Gribodo G. \& Kriechbaumer G. (eds) Rassegna degl'Imenott eri raccolti nel Mozambico dal Cav. Fornasini esistenti nel Museo Zoologico della R. Università di Bologna. Memorie della R. Accademia delle Science dell'Istituto di Bologna, Series 5 4: 111-156.

Gu X., Fu Y.X. \& Li W.H. 1995. Maximum likelihood estimation of the heterogeneity of substitution rate among nucleotide sites. Molecular Biology and Evolution 12 (4) : 546-557.

Guérin-Méneville F.E. 1843. Description de quelques espèces d'Hyménoptères fouisseurs de l'ancien continent, avec des observations sur plusieurs Pompiles nouveaux figurés dans la grande description d'Egypte et restés jusqu'ici inconnus. Magasin de Zoologie, d'Anatomie comparée et de Palceontologie, $2^{e}$ Série 5: 1-8.

Guiglia D. 1939. Imenotteri aculeati raccolti in Eritrea e in Etiopia dal Ten. Colon. Dr. Giorgio Invrea. Memorie della Società entomologica italiana 17: 188-202.

Hafner M.S., Sudman P.D., Villablanca F.X., Spradling T.A., Demaster J.W. \& Nadler S.A. 1994. Disparate rates of molecular evolution in cospeciating hosts and parasites. Science 265: 1087-1090. https://doi.org/10.1126/science.8066445

Haig S.M., Beever E.A., Chambers S.M., Draheim H.M., Dugger B.D., Dunham S., Elliott-Smith E., Fontaine J.B., Kesler D.C., Knaus B.J., Lopes I.F., Loschl P., Mullins T.D. \& Sheffield L.M. 2006. Taxonomic considerations in listing subspecies under the US Endangered Species Act. Conservation Biology 20 (6): 1584-1594. https://doi.org/10.1111/j.1523-1739.2006.00530.x

Hall T.A. 1999. BioEdit: a user-friendly biological sequence alignment editor and analysis program for Windows 95/98/NT. Nucleic Acids Symposium Series 41: 95-98.

Hasegawa M., Kishino H. \& Yano T. 1985. Dating of human-ape splitting by a molecular clock of mitochondrial DNA. Journal of Molecular Evolution 22 (2): 160-174.

https://doi.org/10.1007/BF02101694

Hensen R.V. 1991. Review of Malesian Sphecina (Hymenoptera, Sphecidae, Sphecinae). Tijdschrift voor Entomologie 134: 9-30.

Kalyaanamoorthy S., Minh B.Q., Wong T.K.F., Haeseler A. von \& Jermiin L.S. 2017. ModelFinder: fast model selection for accurate phylogenetic estimates. Nature Methods 14: 587-589.

https://doi.org/10.1038/nmeth.4285

Kirby W.F. 1900. Descriptions of the new species of Hymenoptera. Bulletin of the Liverpool Museums under the City Council 3: 13-24.

Kohl F.F. 1890. Die Hymenopterengruppe der Sphecinen. I. Monographie der natürlichen Gattung Sphex Linné (sens. lat.). Annalen des K. K. Naturhistorischen Hofmuseums 5 (2): 77-194. Available from https://www.biodiversitylibrary.org/page/4046912 [accessed 22 Dec. 2021].

Kohl F.F. 1895. Zur Monographie der natürlichen Gattung Sphex Linné. Annalen des K. K. naturhistorischen Hofmuseums 10: 42-74. https://doi.org/10.5962/bhl.part.17608

Kohl F.F. 1898. Über neue Hymenopteren. Természetrajzi Füzetek 21: 325-367.

Kohl F.F. 1906. Zoologische Ergebnisse der Expedition der kaiserlichen Akademie der Wissenschaften nach Südarabien und Sokótra im Jahre 1898-1899. Hymenopteren. Denkschriften der kaiserlichen Akademie der Wissenschaften in Wien, Mathematisch-Naturwissenschaftliche Klasse 71: 169-301. 
Kohl F.F. 1909. Sphegiden und Pompiliden von Madagaskar, den Comoren und Ostafrika. In: Voeltzkow A. (ed.) Reise in Ostafrika in den Jahren 1903-1905 mit Mitteln der Hermann und Elise geb. Heckmann Wentzel-Stiftung ausgeführt. Wissenschaftliche Ergebnisse. Band 2. Systematische Arbeiten. Issue 4: 369-378. E. Schweitzerbartsche Verlagsbuchhandlung Nägele \& Dr Sproesser, Stuttgart.

Kohl F.F. 1913. Neue Pompiliden und Sphegiden vom belgischen Congogebiete. Revue zoologique africaine 3: 182-209.

Leclercq J. 1955. Hymenoptera Sphecoidea (Sphecidae I. Subfam. Sphecinae). Exploration du Parc national de l'Upemba 34:1-137.

Leclercq J. 1961. Sur les Sphex africains du groupe haemorrhoidalis Fabricius (Hymenoptera Sphecidae). Bulletin de l'Institut agronomique de Gembloux et des Stations de Recherche de Gembloux 29: 323-327.

Lepeletier de Saint Fargeau A.L.M. 1845. Histoire naturelle des Insectes. Hyménoptères. Volume 3. Librairie Encyclopédique de Roret, Paris.

Lindahl T. 1993. Instability and decay of the primary structure of DNA. Nature 362 (6422): 709-715. https://doi.org/10.1038/362709a0

Magretti P. 1884. Risultati di raccolte imenotterologiche nell'Africa orientale. Annali del Museo civico di Storia naturale di Genova 21: 523-636.

Magretti P. 1898. Imenotteri della seconda spedizione di Don Eugenio dei Principi Ruspoli nei paesi Galla e Somali. Annali del Museo civico di Storia naturale di Genova 39: 25-56.

Mayr E. 1942. Systematics and the Origin of Species from the Viewpoint of a Zoologist. Columbia University Press, New York.

Mayr E. 1982. Of what use are subspecies? The Auk 99 (3): 593-595.

Menke A.S. 1962. Notes on two species of sphecine wasps described by H.T. Fernald from South America (Hymenoptera: Sphecidae). The Pan-Pacific Entomologist 38: 63.

Mocsáry A. 1883. Hymenoptera nova Europaea et exotica - Európai és másföldi új hártyaröpűek. Értekezések a Természettudományok Köréböl 13 (11): 1-72.

Nylander J.A.A. 2004. MrModeltest v2. Program distributed by the author. Evolutionary Biology Centre, Uppsala University, Uppsala, Sweden.

Oehlke J. \& Wudowenz J. 1973. Katalog der in den Sammlungen des ehemaligen Deutschen Entomologischen Institutes aufbewahrten Typen - X. Hymenoptera. Sphecidae. Beiträge zur Entomologie 23 (5-8): 421-426.

Ohl M. 1996. Die phylogenetischen Beziehungen der Sphecinae (Hymenoptera: Apoidea: "Sphecidae") aufgrund morphologischer Merkmale des Exoskeletts. Zooloogische Beiträge (AGE) 37: 3-40.

Pérez J. 1895. Mission scientifique de M. Ch. Alluaud aux îles Séchelles (Mars-Avril-Mai 1892). $2^{\mathrm{e}}$ Mémoire. Hyménoptères. Annales de la Société entomologique de France 64: 205-212.

Posada D. \& Crandall K.A. 1998. Modeltest: testing the model of DNA substitution. Bioinformatics 14 (9): 817-818. https://doi.org/10.1093/bioinformatics/14.9.817

Pulawski W.J. 2020. Catalog of Sphecidae. California Academy of Sciences, San Francisco, CA. Available from https://www.calacademy.org/scientists/projects/catalog-of-sphecidae [accessed 27 Apr. 2020].

Rogenhofer A.F. 1889. Afrikanische Schmetterlinge des k.k. naturhistorischen Hofmuseums. I. Annalen des naturhistorischen Museums in Wien 4: 547-554. 
Schmid-Egger Ch. 2014. Order Hymenoptera, families Crabronidae and Sphecidae. Further records and descriptions of new species. Arthropod Fauna of the UAE 5: 521-631.

Schmid-Egger Ch., Straka J., Ljubomirov T., Blagoev G.A., Morinière J. \& Schmidt S. 2018. DNA barcodes identify 99 per cent of apoid wasp species (Hymenoptera: Ampulicidae, Crabronidae, Sphecidae) from the Western Palearctic. Molecular Ecology Resources 19 (2): 476-484.

https://doi.org/10.1111/1755-0998.12963

Smith F. 1873. Descriptions of new species of fossorial Hymenoptera in the collection of the British Museum, and of a species of the rare genus Iswara, belonging to the family Dorylidae. The Annals and Magazine of Natural History, Series 4 12: 253-260 (Sep.), 291-300 (Oct.), 402-415 (Oct.).

https://doi.org/10.1080/00222937308680752

Souza C.A.S., Prezoto F., Lima M.S. \& Pederassi J. 2015. Sexual behavior of the digger wasp Sphex ingens Smith (Hymenoptera, Sphecidae). Revista brasileira de Entomologia 59 (2): 107-112.

https://doi.org/10.1016/j.rbe.2015.03.005

Strand E. 1916. Über einige exotische Crabroniden der Gattungen Sphex und Sceliphron im Deutschen Entomologischen Museum. Archiv für Naturgeschichte 81A (11): 98-109.

Swofford D.L. 2003. PAUP*. Phylogenetic Analysis Using Parsimony (*and Other Methods). Version 4. Sinauer Associates, Sunderland, MA.

Taschenberg E.L. 1869. Die Sphegidae des zoologischen Museums der Universität in Halle. Zeitschrift für die gesammten Naturwissenschaften 34: 407-435.

Tavaré S. 1986. Some probabilistic and statistical problems in the analysis of DNA sequences. Lectures on Mathematics in the Life Sciences, American Mathematical Society 17: 57-86.

Trifinopoulos J., Nguyen L.-T., Haeseler A. von \& Minh B.Q. 2016. W-IQ-TREE: a fast online phylogenetic tool for maximum likelihood analysis. Nucleic Acids Research 44: W232-W235.

https://doi.org/10.1093/nar/gkw256

Turner R.E. 1910. Additions to our knowledge of the fossorial wasps of Australia. Proceedings of the Zoological Society of London 80 (1): 253-355. https://doi.org/10.1111/j.1096-3642.1910.tb01895.x

Turner R.E. 1912. Notes on fossorial Hymenoptera. - X. On new species from the Oriental and Ethiopian Regions. The Annals and Magazine of Natural History, Series 8 10: 361-377.

https://doi.org/10.1080/00222931208693249

Turner R.E. 1918. Notes on fossorial Hymenoptera. - XXXV. On new Sphecoidea in the British Museum. The Annals and Magazine of Natural History, Series 9 1: 356-364.

https://doi.org/10.1080/00222931808562327

Turner R.E. 1919. Notes on fossorial Hymenoptera. - XXXVII. On some Sphecinae in the British Museum. The Annals and Magazine of Natural History, Series 9 3: 393-397.

https://doi.org/10.1080/00222931908673834

Udvardy M.D.F. 1975. A Classification of the Biogeographical Provinces of the World. Volume 8. International Union for Conservation of Nature and Natural Resources, Morges, Switzerland.

Van der Vecht J. 1961. Hymenoptera Sphecoidea Fabriciana. Zoologische Verhandelingen 48: 1-85.

Van der Vecht J. 1973. Contribution to the taxonomy of the Oriental and Australian Sphecini (Hymenoptera, Sphecidae). Proceedings, Koninklijke nederlandse Akademie van Wetenschappen, Series C 76: 341-353. 
Walker F. 1871. A List of Hymenopterous Insects Collected by J.K. Lord, Esq. in Egypt, in the Neighbourhood of the Red Sea, and in Arabia. With Descriptions of the New Species. E.W. Janson, London. https://doi.org/10.5962/bhl.title.8818

Yang Z. 1994. Maximum likelihood phylogenetic estimation from DNA sequences with variable rates over sites: approximate methods. Journal of Molecular Evolution 39: 306-314.

https://doi.org/10.1007/BF00160154

Manuscript received: 6 May 2021

Manuscript accepted: 15 November 2021

Published on: 23 February 2022

Topic editor: Nesrine Akkari

Section editor: Gavin Broad

Desk editor: Danny Eibye-Jacobsen

Printed versions of all papers are also deposited in the libraries of the institutes that are members of the EJT consortium: Muséum national d'histoire naturelle, Paris, France; Meise Botanic Garden, Belgium; Royal Museum for Central Africa, Tervuren, Belgium; Royal Belgian Institute of Natural Sciences, Brussels, Belgium; Natural History Museum of Denmark, Copenhagen, Denmark; Naturalis Biodiversity Center, Leiden, the Netherlands; Museo Nacional de Ciencias Naturales-CSIC, Madrid, Spain; Real Jardín Botánico de Madrid CSIC, Spain; Zoological Research Museum Alexander Koenig, Bonn, Germany; National Museum, Prague, Czech Republic.

Supplementary file 1. Further data on voucher specimens of Sphex Linnaeus, 1758 used for DNA sequencing (see also Table 2). 